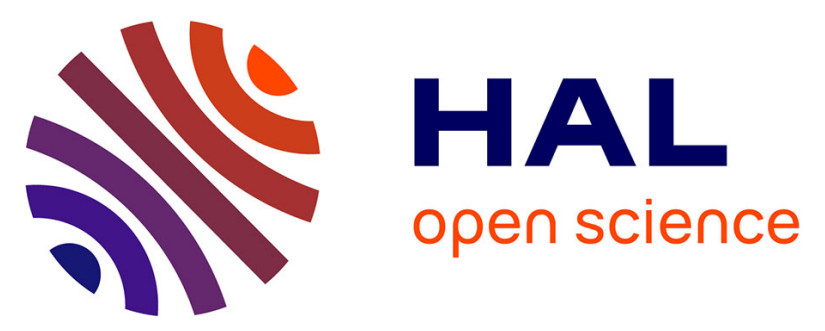

\title{
Rapport sur les travaux de l'école française en Grèce en 1987
}

\author{
Michèle Brunet, Anne Pariente, Pierre Aupert, Jean-Charles Moretti, \\ Evangelos Pentazos, Vincent Déroche, François Queyrel, Katérina Péristeni, \\ René Treuil, Jacques-y Perreault, et al.
}

\section{To cite this version:}

Michèle Brunet, Anne Pariente, Pierre Aupert, Jean-Charles Moretti, Evangelos Pentazos, et al.. Rapport sur les travaux de l'école française en Grèce en 1987. Bulletin de Correspondance Hellenique, 1988, 112 (2), pp.697-791. 10.3406/bch.1988.6749 . hal-01715551

\section{HAL Id: hal-01715551 \\ https://hal.science/hal-01715551}

Submitted on 16 Apr 2018

HAL is a multi-disciplinary open access archive for the deposit and dissemination of scientific research documents, whether they are published or not. The documents may come from teaching and research institutions in France or abroad, or from public or private research centers.
L'archive ouverte pluridisciplinaire $\mathbf{H A L}$, est destinée au dépôt et à la diffusion de documents scientifiques de niveau recherche, publiés ou non, émanant des établissements d'enseignement et de recherche français ou étrangers, des laboratoires publics ou privés.

\section{(1)(1) $\$(0)$}

Distributed under a Creative Commons Attribution - NonCommercial - ShareAlikel 4.0 


\section{Rapport sur les travaux de l'école française en Grèce en 1987}

Anne Pariente, Pierre Aupert, Jean-Charles Moretti, Evangelos Pentazos, Vincent

Déroche, François Queyrel, Michel Sève, Katérina Péristeni, René Treuil, Jacques-Y Perreault, Jean-Yves Empereur, Angeliki Simossi, Yves Grandjean, Haïdo KoukouliChryssantakhi, Tony Kozeli, François Salviat, Michèle Brunet, Roland Étienne, Alexandre Farnoux, Philippe Fraisse, Gérard Siebert, Françoise Alabe, Hervé Duchêne

\section{Citer ce document / Cite this document :}

Pariente Anne, Aupert Pierre, Moretti Jean-Charles, Pentazos Evangelos, Déroche Vincent, Queyrel François, Sève Michel, Péristeni Katérina, Treuil René, Perreault Jacques-Y, Empereur Jean-Yves, Simossi Angeliki, Grandjean Yves, KoukouliChryssantakhi Haïdo, Kozeli Tony, Salviat François, Brunet Michèle, Étienne Roland, Farnoux Alexandre, Fraisse Philippe, Siebert Gérard, Alabe Françoise, Duchêne Hervé. Rapport sur les travaux de l'école française en Grèce en 1987. In: Bulletin de correspondance hellénique. Volume 112, livraison 2, 1988. pp. 697-791;

doi : 10.3406/bch.1988.6749

http://www.persee.fr/doc/bch_0007-4217_1988_num_112_2_6749

Document généré le 18/05/2016 


\section{RAPPORT SUR LES TRAVAUX DE L'ÉCOLE FRANÇAISE EN GRÈCE EN 1987*}

\section{ARGOS}

\section{1. - Agora}

La campagne de fouilles 1987 s'est déroulée du 20 juillet au 8 août avec quatre équipes d'ouvriers, puis du 10 au 15 août avec une seule équipe. $Y$ ont pris part des étudiants de l'Université de Nancy II (M. André et E. Lips) et de l'Université de Frihourg (Suisse) (M. della Santa et. M.-L. Pichonnaz). Les monnaies ont èté nettoyées par 0 . Didelot et $\mathrm{S}$. Sanders. Les résultats (poursuite de l'exploration des bâtiments de l'agora archaique dans la partie Sud du portique classique, étude du \& dromos») en ayant été exposés dans la Chronique du $B C H 111$ (1987), p. 585-591, aucun rapport ne sera publié en 1988.

\section{2. - Terrain Karmoyannis \\ par Anne Pariente}

I. La troisième campagne de fouilles sur le terrain Karmoyannis s'est déroulée du 17 juin au 17 juillet 1987, avec quatre équipes d'ouvriers, sous la direction du contremaitre D. Iliopoulos; ont également participé à la fouille, en qualité d'assistantes, V. Borne, ètudiante à l'Université de Saint-Etienne, et M. Oravec, ètudiante à l'Universitè de Reims, qui a par ailleurs commencé à dresser le catalogue de l'apothèque de céramique du Musée. K. Kolokotsas a effectué relevés et plans; le nettoyage du matériel métallique (petits objets et monnaies - dont l'identification est due à $\mathrm{O}$. Picard) a été confiè à $\mathrm{O}$. Didelot et $\mathrm{S}$. Sanders.

(*) De septembre 1986 à septembre 1987, le personnel scientifique de l'École comprenait : Olivier Picard, directeur; Jean-Yves Empereur, secrétaire général ; Gilles Touchais, bibliothécaire; Vincent Déroche et Hervé Duchêne, membres en $4^{e}$ année; Michèle Brunet et Anne Pariente, membres en $3^{e}$ année ; Françoise Alabe et Jean-Charles Moretti, membres en $2^{*}$ année; Alexandre Farnoux, membre en $1^{\text {re }}$ année; au $1^{\text {er }}$ septembre 1987 , Marie-Dominique Nenna et Denis Rousset ont été nommés membres; Christiane Tytgat était membre belge en $5^{e}$ année; Thierry Petit, membre belge en $1^{\text {re }}$ année; Jacques Y. Perreault, membre canadien en $3^{e}$ année; Sylvie Muller, a été accueillie comme membre suisse en $\mathrm{I}^{\text {re }}$ année. 


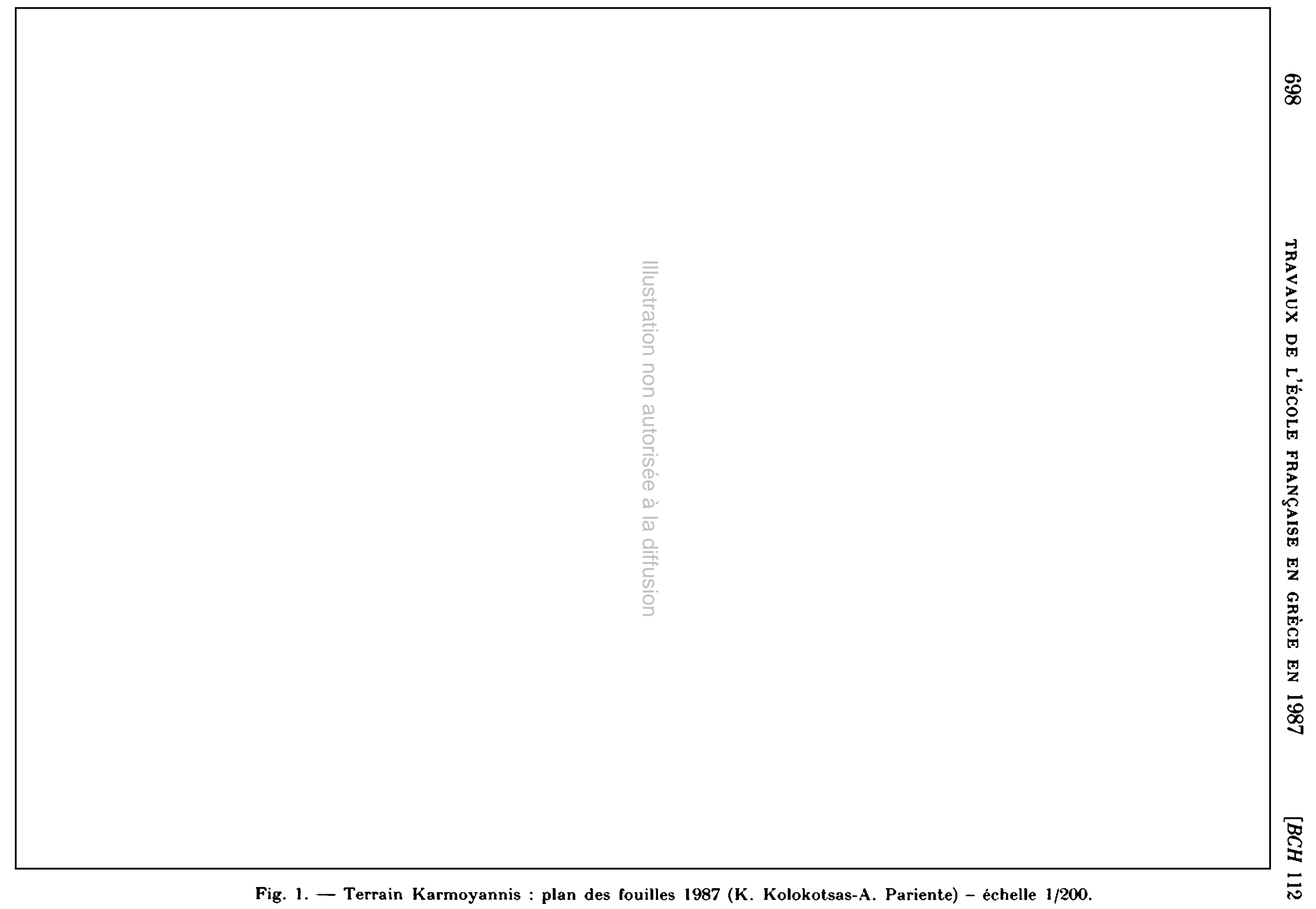


Cette campagne avait pour objectifs principaux :

1) d'ètudier l'emplacement de l'imposant foyer mis au jour en $1986^{1}$, et les éventuels vestiges d'occupation antérieure;

2) de déterminer l'extension et la nature de la construction au sol bétonné, partiellement mise au jour lors des fouilles $1985^{2}$ en A.N-AO-AP 793, et lors des fouilles 1986 en AN-AO-AP $78^{4}$.

Ont èté ouverts dans ce but des sondages à l'extrémité Ouest de la fosse de l'enclos-foyer - dont on a poursuivi aussi l'exploration de la limite Sud, dans la partie Nord des carrés AQ 77-78 -, ainsi que dans les carrés AN-AO 76, AO 77, AM 78 et enfin AL 78, pour tenter de préciser la chronologie d'un nouveau bâtiment mis au jour dans la partie. Nord du terrain; la fouille avait du être précédée par la destruction d'une maison, située le long de l'actuelle rue du Thèàtre, et par un décapage à la pelle mécanique de la partie de terrain ainsi dégagée (ifg. 1).

\section{LA NÉCROPOI.E RÉCENTE.}

Tous les sondages ouverts en 1987 (sauf ceux de l'enclos-foyer) ont livré leur quota de sépultures en pleine terre, portant à trente-huit le total des tombes découvertes en trois années de fouilles; comme d'ordinaire dans cette nécropole, les squelettes mis au jour, orientés Sud-Ouest/Nord-Est, étaient ensevelis sans la moindre offrande, à des cotes variant entre $+10,89$ et $+10,20$.

En l'état actuel des recherches, l'indice chronologique le plus fiable concernant ces tombes reste celui découvert en 1968 dans les Thermes B, où une monnaie de Georges Ier, de 1869, avait été trouvée dans la couche au bas de laquelle affleuraient les fosses funéraires ${ }^{6}$.

Deux des tombes dégagées en 1987 permettent du moins de supposer des variantes dans le mode d'ensevelissement : autour de la tête et du buste d'un des squelettes ont été retrouvés sept clous en fer, qui ne pouvaient servir qu'à fixer les planches d'un cercueil de fortune - dont toute trace avait disparu ; sous la tête d'un autre squelette se trouvait d'autre part une poignée de fer de forme oblongue, qui pourrait avoir retenu un plan de tissu servant à descendre le corps dans la fosse.

\section{L'ENCLOS-FOYER.}

a. Le sondage implanté à l'extrémité Ouest de l'enclos, sur 2,50 $\mathrm{m}$ de long, du bord Nord au bord Sud de la fosse?, et 1,20 m de large, a livré d'abord les restes, fichés dans le sol du foyer, d'un vase placé à l'envers dans une cuvette creusée à cet effet $($ cote $+9,42)$ : il s'agit de l'embouchure et du col d'un cratère en céramique commune d'époque probablement hellénistique ou romaine, de destination funéraire puisqu'il contenait encore quelques ossements calcinés (fig. 2). Vraisemblablement en place avant l'installation du foyer, sa panse a dú être arrachée lors du creusement de la fosse.

Sous la croùte de terre très cuite de quelques centimètres d'épaisseur seulement qui constituait le fond de la fosse du foyer, le sondage a été poursuivi jusqu'à la cote $+7,69$, à travers une succession de couches alluvionnaires, composées alternativement de gravier et d'argile rougeâtre à filons gris, dont les deux plus profondes ètaient absolument stériles (fig. 3).

La présence exclusive de ces couches d'alluvionnement permet de conclure que l'enclos-foyer n'est pas le dernier ètat d'un foyer existant antérieurement ${ }^{8}$ sur le mème emplacement, mais qu'il fut installè en un lieu

(1) Cf. Chronique des fouilles 1986, BCH 111 (1987), p. 595.

(2) Cf. BCH 110 (1986), p. 767.

(3) Pour l'emplacement des carrés, se reporter au plan fig. 1, p. 592.

(4) Cf. BCH 111 (1987), p. 591-595.

(5) Cf. $B C H 93$ (1969), p. 978.

(6) Une inhumation dans un cercueil avait déjà été découverte au Nord du "portique Sud": cf. $B C H 99$ (1975), p. 704.

(7) Ce sondage a également permis de déchausser l'une des bornes de la limite Nord de l'enclos : sa partie inférieure, plantée dans le sol, plus large que la partie visible et beaucoup plus grossièrement dressée, est haute de $0,45 \mathrm{~m}$.

(8) Contrairement à ce que la prudence me suggérait dans la chronique des fouilles 1986 (cf. BCH 111 [1987], p. 595 n. 4), il ne me semble plus totalement invraisemblable que ce foyer du Ive s. ap. J.-C. - siècle de 


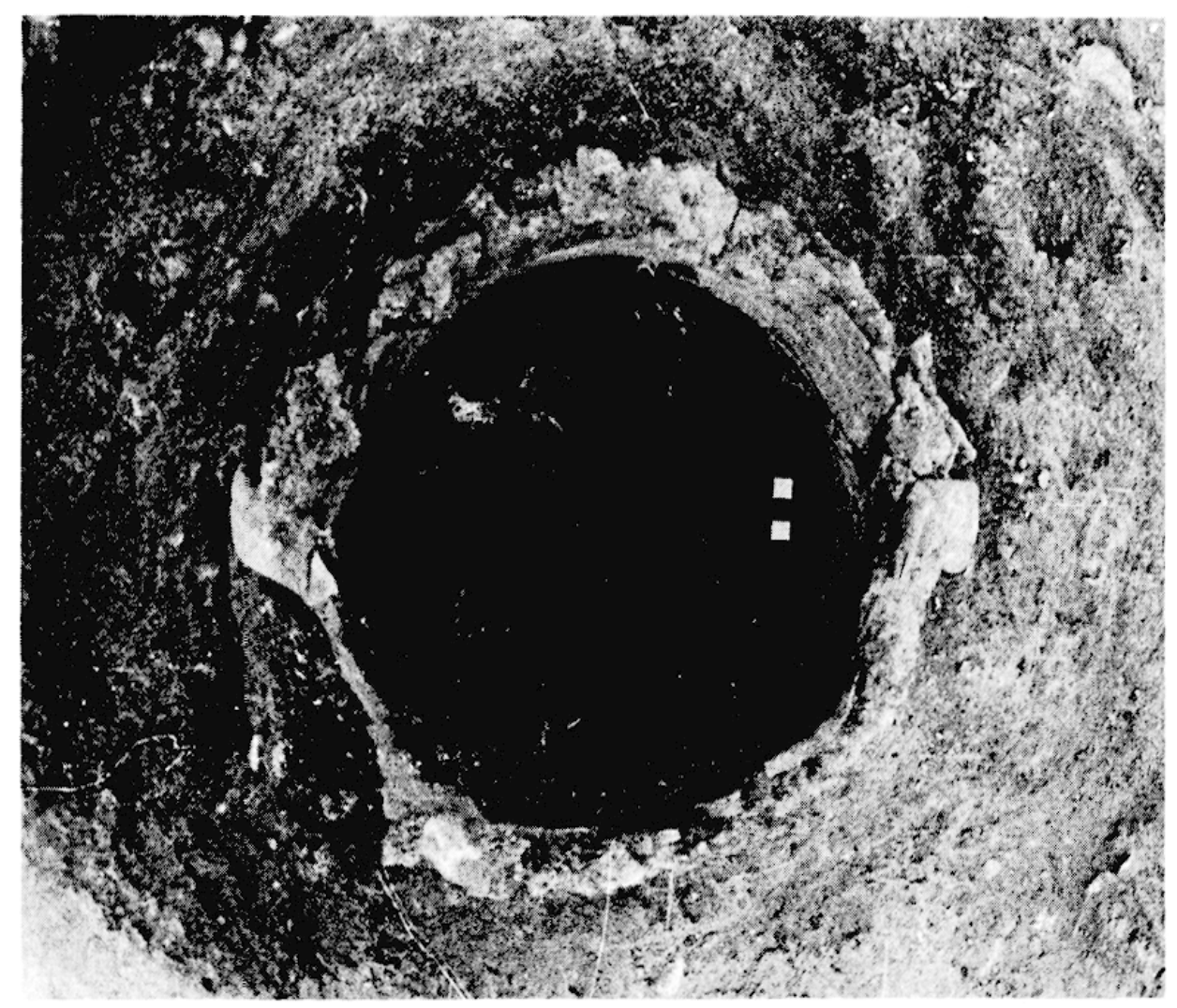

Fig. 2. - Restes d'un cratère funéraire fichès dans le sol du foyer.

vierge de toute occupation - en dehors du cratère funéraire dont il reste à expliquer la présence. La minceur de la couche de terre cuite sous l'effet de la chaleur dégagée par le brasier semble d'autre part exclure que la fosse ait été utilisée de façon répétée pour de nombreuses flambées.

b. Afin de terminer le dégagement de la limite Sud de l'enclos, on a fouillé le quart Sud-Est de $A Q 77$ et la moitié Ouest de $A Q 78$. Recouverte par la couche de remblais brun gris qui s'étend sur l'ensemble du terrain, une neuvième borne, en place dans la couche brun orangé à gravier (cote $+9,95$ ) qui constitue le sol aux alentours immédiats du foyer et s'abaisse légèrement en direction du Sud, a été mise au jour en $A Q 77^{\circ}$ : elle est la première à présenter des cavités (destinèes à l'insertion de rondins de bois) sur deux faces perpendiculaires (faces Nord et Est dans la disposition actuelle), preuve que l'enclos d'origine ètait bien un dispositif fermé.

La fouille s'est étendue suffisamment vers l'Est pour que, les bornes de la limite Sud étant écartées de façon constante de $1,20 \mathrm{~m}$ à $1,40 \mathrm{~m}$, il paraisse assuré que cette dernière borne ètait située à l'angle Sud-Est de l'enclos tel qu'il fut reconstitué autour du foyer. Le doute persiste en revanche quant à son plan d'origine, car la borne tombée dans la perturbation byzantine ${ }^{10}$ s'est révélée ètre la troisième à présenter des cavités sur trois de ses faces.

\section{La structure AU SOL BÉtonNÉ.}

La campagne 1987 a apporté d'importantes précisions tant sur l'extension que sur la fonction de cette construction.

remise en vigueur du paganisme et de grands travaux de restructuration et de remaniements architecturaux sur

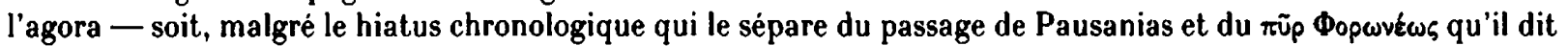
avoir vu brûler dans le sanctuaire d'Apollon Lycien (II, 19,5), la dernière version, à grande échelle et en un lieu nouveau, de l'antique coutume paienne dont les Argiens honoraient leur ancêtre mythique.

(9) Elle ne figure pas sur le plan fig. 1.

(10) Cf. BCH 111 (1987), p. 595 et fig. 10. 


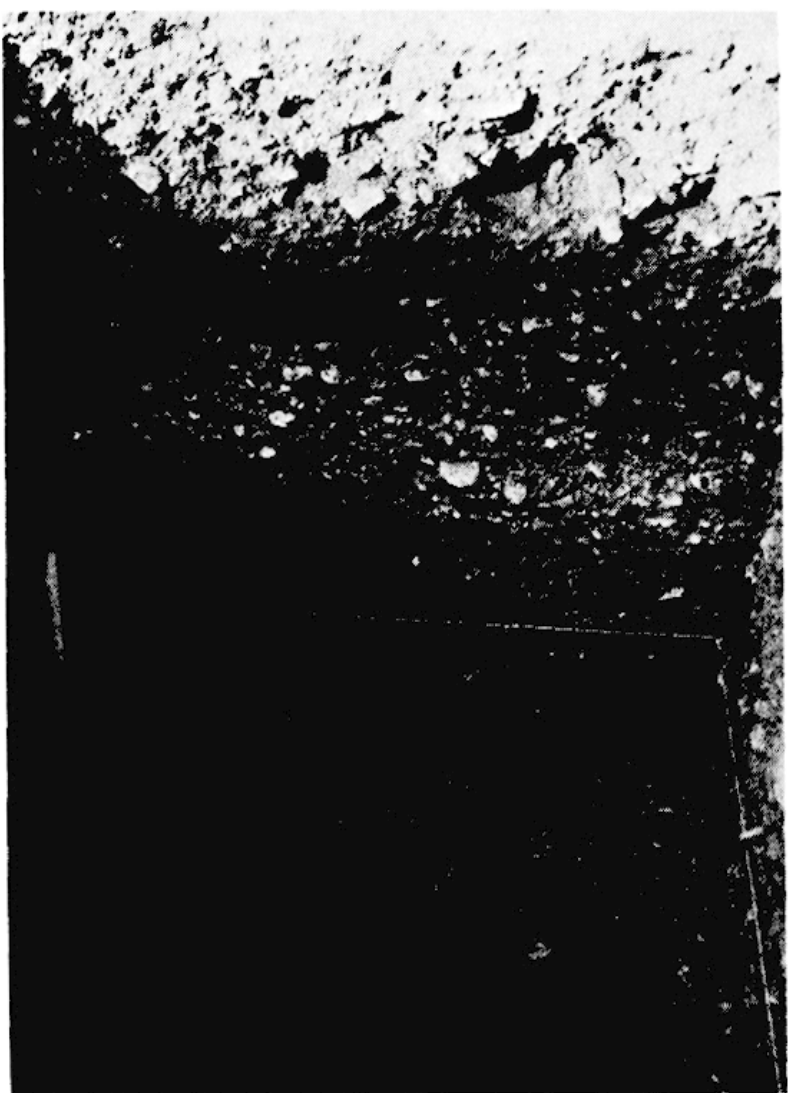

Fig. 3. - Couches d'alluvions sous le sol du foyer (berme Sud du sondage).

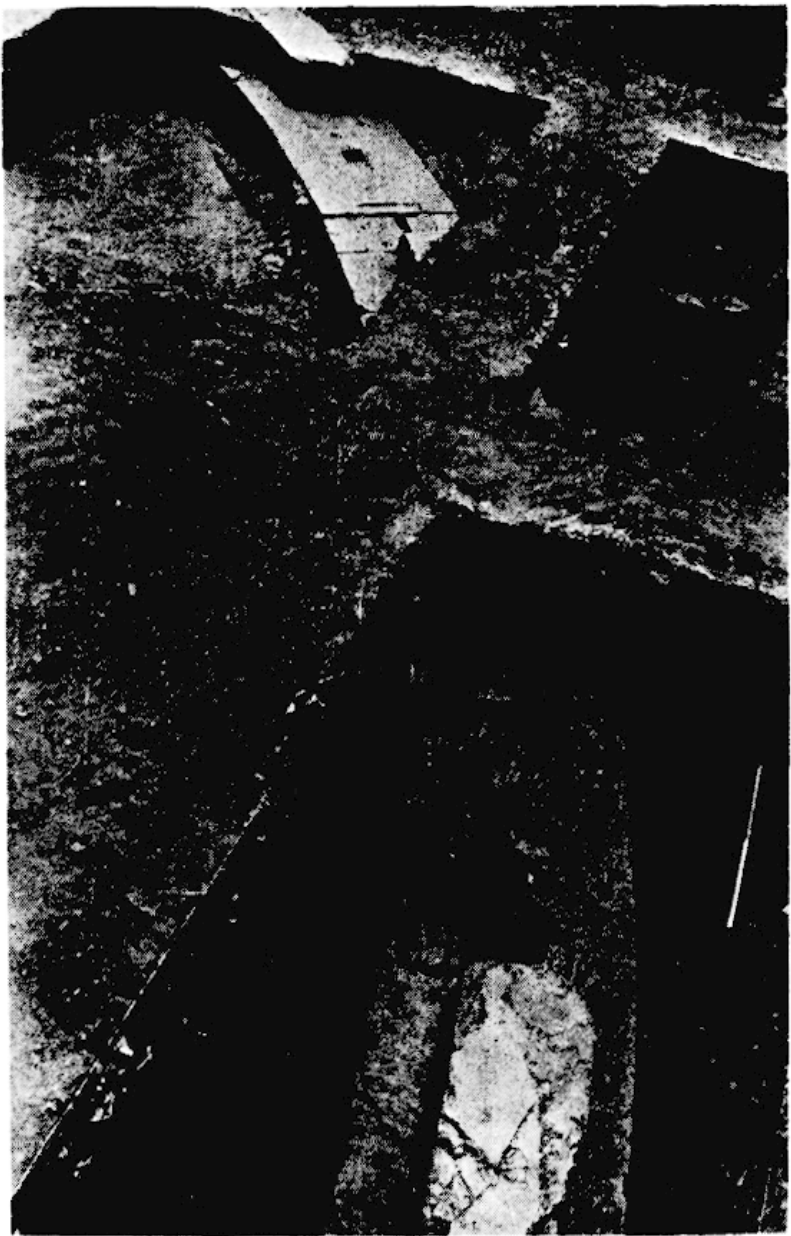

Fig. 4. - Branche Ouest de la construction en arc outrepassé (AN 76/AO 77 - vers le Sud).

a. Sa limite Ouest, mise au jour dans les carrés AN-AO 76 et AO 77, apparaissait déjà en 1986 dans l'angle Nord-Est de AP 77'. Elle forme un large arc de cercle (fig. 4), qui se prolonge avec l'empreinte laissée au bord de la perturbation byzantine (AP 78) par les blocs disparus (fig. 5); si, comme y autorise la similarité de structure ${ }^{12}$, on le met en relation avec une construction en gradins mise partiellement au jour il y a une quinzaine d'années par Ch. Kritzas dans un terrain situé légèrement à l'Est du terrain Karmoyannis, on restitue pour le plan du bátiment au moins un demi-cercle, qui, parce qu'il vient buter contre la krépis dont il sera question ci-dessous, forme en réalité un arc outrepassé, de $28 \mathrm{~m}$ de diamètre.

Cette limite est constituée de degrés de calcaire (deux au Nord [cotes $+10,45$ et $+10,21$, sur une euthyntéria en calcaire, un seul au Sud [cote $+10,22]$, sur une assise de fondation en poros), formés de deux panneresses et disposés en gradins cóté intérieur comme côté extérieur.

- La joue des degrés présente une partie usée (par les pas) dont la largeur varie de 0,293 m (degré inférieur) à $0,25 \mathrm{~m}$ (degré supérieur), précédant une partie plus étroite assez grossièrement piquetée. Le chant, côté extérieur des gradins, est finement ravalé à la pointe, et l'arête horizontale du degré inférieur taillée en biseau. Le seul bout visible, celui du dernier bloc en place au bord Nord-Ouest de la perturbation (en AP 77), est dotè d'une anathyrose en gamma, sur toute la hauteur de l'arête verticale, et la largeur de la marche ${ }^{13}$. Les

(11) Cf. $B C H 111$ (1987), p. 592 et fig. 11.

(12) Je remercie l'Éphorie des Antiquités de Nauplie de m'en avoir montré des photographies.

(13) Largeur du bandeau horizontal : $7,5 \mathrm{~cm}$. - du bandeau vertical :5 cm. 
panneresses, dont la face arrière est le plus souvent irrégulière, sont séparées par un blocage de terre et, en certains endroits, d'éclats de taille de calcaire.

- Les degrés sont surmontés d'une dernière assise plus haute (cote $+10,75 /+10,81)$, disposée à cheval sur la partie piquetée du lit d'attente des deux blocs du niveau inférieur. Toute la joue en est également usée; le chant extérieur est couronné d'une moulure (bandeau surmontant un quart de rond souligné par une baguette en faible relief). Le bout de cette assise porte une anathyrose en $\mathrm{pi}^{14}$.

En AO 77, l'assise supérieure est interrompue sur 1,20 m de long, laissant ainsi un passage sur un seuil monolithe de 2,25 $\mathrm{m}$ de long, d'une largeur équivalente à celle des deux degrés intérieur et extérieur (fig. 6) : uniformément usé par les pas, il comporte une mortaise rectangulaire (de $0,17 \times 0,14 \times 0,15 \mathrm{~m}$ ), vraisemblablement destinée à recevoir un poteau de bois appartenant à un système - symbolique - de fermeture. Peut-être faut-il restituer un second passage, disposé symétriquement..

- Le chant des degrés côté intérieur n'est pas travaillé de façon uniforme : la partie supérieure du chant est ravalée sur 4 à $10 \mathrm{~cm}$ seulement, tout le reste du chant, en débord, n'étant que très grossièrement dressé (fig. 7). Le sol d'origine, dont rien n'a subsisté, devait cacher la partie basse des degrés, à partir de la cote $+10,25$, donc beaucoup plus haut que le sol bétonné (cote moyenne $+9,75$ ).

Au centre de cette vaste construction se trouve la fondation - s'enfonçant sous la limite Est du terrain contre laquelle vient buter le sol bétonné ${ }^{15}$. L'assise encore en place à son angle Sud-Ouest (cote $\left.+9,98\right)$ est constituée de cinq blocs de poros bien appareillés (sur une longueur de $3,20 \mathrm{~m}$ ), séparés de blocs de mème nature plus au Nord (sur une longueur de $3,50 \mathrm{~m}$ jusqu'à l'aplomb de la krépis dont il sera question ci-dessous) par un intervalle de $2 \mathrm{~m}$, obstrué lors d'un remaniement avec deux blocs du socle de l'autel à triglyphes bas auquel appartenaient le bloc triglyphe-métopes mis au jour en $1985^{16}$, ainsi qu'un bloc voisin, provenant de son couronnement. Le profil et les dimensions de ces nouveaux éléments d'autel à triglyphes bas le différencient nettement de l'autel de même type identifié par G. Roux comme autel d'Apollon Lycien ${ }^{17}$.

Sur la partie Nord de la base se trouvent remployés également trois blocs de calcaire ${ }^{18}$ provenant d'une base honorifique, dont le plus méridional porte une inscription de trois lignes difficilement déchiffrables (fig. 8) :

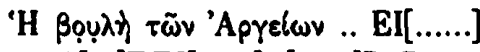

$$
\begin{aligned}
& \Delta[\ldots] \mathrm{E} \Pi ![\ldots . .] \mathrm{A}[\ldots \ldots . .] \mathrm{B} . \mathrm{B} \text {. }
\end{aligned}
$$

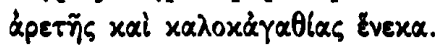

La graphie (apices assez importants, $\mathrm{A}$ à barre brisée, $\Pi$ à barre horizontale débordant légèrement les barres verticales, $\mathrm{K}$ à barres assez courtes), semblable à celle de l'inscription $\mathrm{E} 92^{10}$, pourrait dater de la haute époque impériale ${ }^{20}$.

b. Reste à s'interroger sur la fonction de cette ètonnante construction 22. Sa forme, en arc outrepassé, évoque celle d'un théâtron, et il est tentant d'accorder à l'assise supérieure la fonction qu'un gradin aurait dans un théătre, celle de banc. La capacité de cette assise-banc, qui se développait sur une longueur d'environ $49 \mathrm{~m}$, peut être estimée, sur la base d'une largeur d'environ deux pieds (à peu près $0,60 \mathrm{~m}$ ) pour chaque place, à un peu

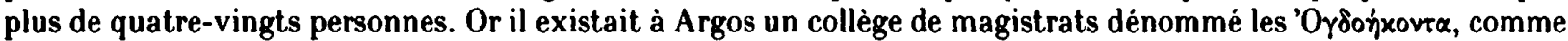
l'attestent de rares témoignages (un passage de Thucydide [V, 47,9], reproduisant les termes du traitè d'alliance entre Athènes, Argos, Elis et Mantinée [420 av. J.-C.], et un décret en l'honneur des Rhodiens [fin Ive-dèbut III s.] ${ }^{22}$ ), d'où il ressort que cette assemblée serait une survivance aristocratique qui n'aurait plus, après l'institution de la $\beta \omega \lambda \alpha$ démocratique, d'attributions que religieuses. La structure en arc outrepassé pourrait être le siège des 'OY $\delta$ b́nxovia, dont les regards convergeaient vers une base sur laquelle il est tentant de rétablir un autel, encore anonyme, où se déroulaient des sacrifices qu'a pu accompagner le spectacle de chœurs évoluant sur l'aire semi-circulaire de quelque $10 \mathrm{~m}$ de large pour laquelle ils constitueraient une excellente raison d'être.

(14) Largeur des bandeaux vertical extérieur et horizontal : $5 \mathrm{~cm}$. - du bandeau vertical intérieur : $4 \mathrm{~cm}$.

(15) Cf. BCH 110 (1986), p. 767.

(16) Cf. $B C H 110$ (1986), p. 767 et fig. 3.

(17) Cf. BCH 77 (1953), p. 116-123.

(18) Longueur : $0,87 \mathrm{~m}$ - largeur : de 0,41 à $0,49 \mathrm{~m}$ - hauteur : de 0,65 à $0,67 \mathrm{~m}$.

(19) Cf. $B C H 102$ (1978), p. 784 et fig. 19.

(20) Je remercie $M$. Sève de son expertise.

(21) Pour une étude plus détaillée, se reporter à mon mémoire de $4^{e}$ année, intitulé Nouvelles découvertes sur l'agora argienne, déposé aux archives de l'EFA.

(22) Cf. Nouveau Choix d'inscriptions grecques (1971), no 8, p. 60-63 (1. 30). 


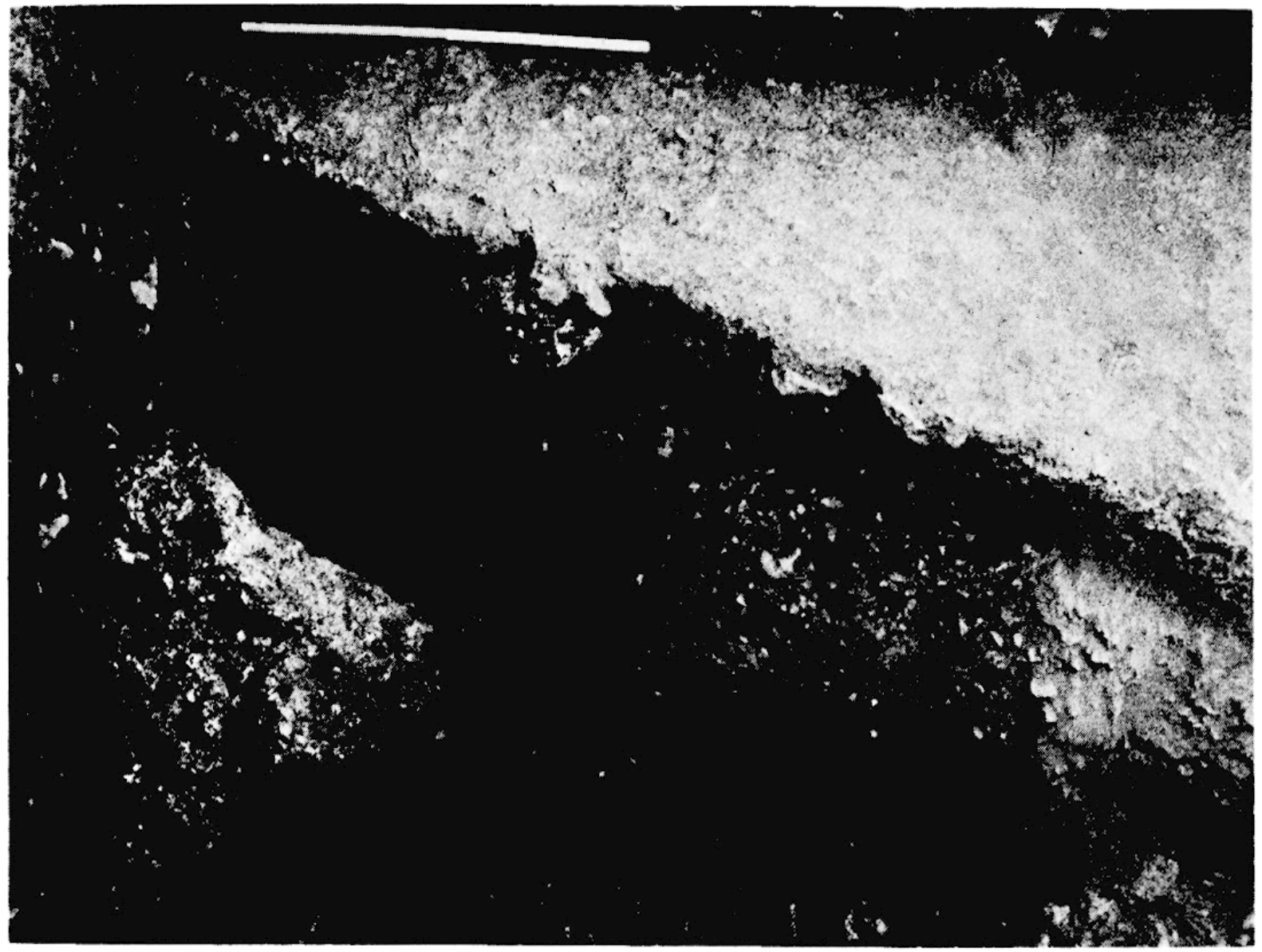

Fig. 5. - Empreinte laissée par les blocs arrachés au bord de la perturbation (AP 78 - vers le Nord).

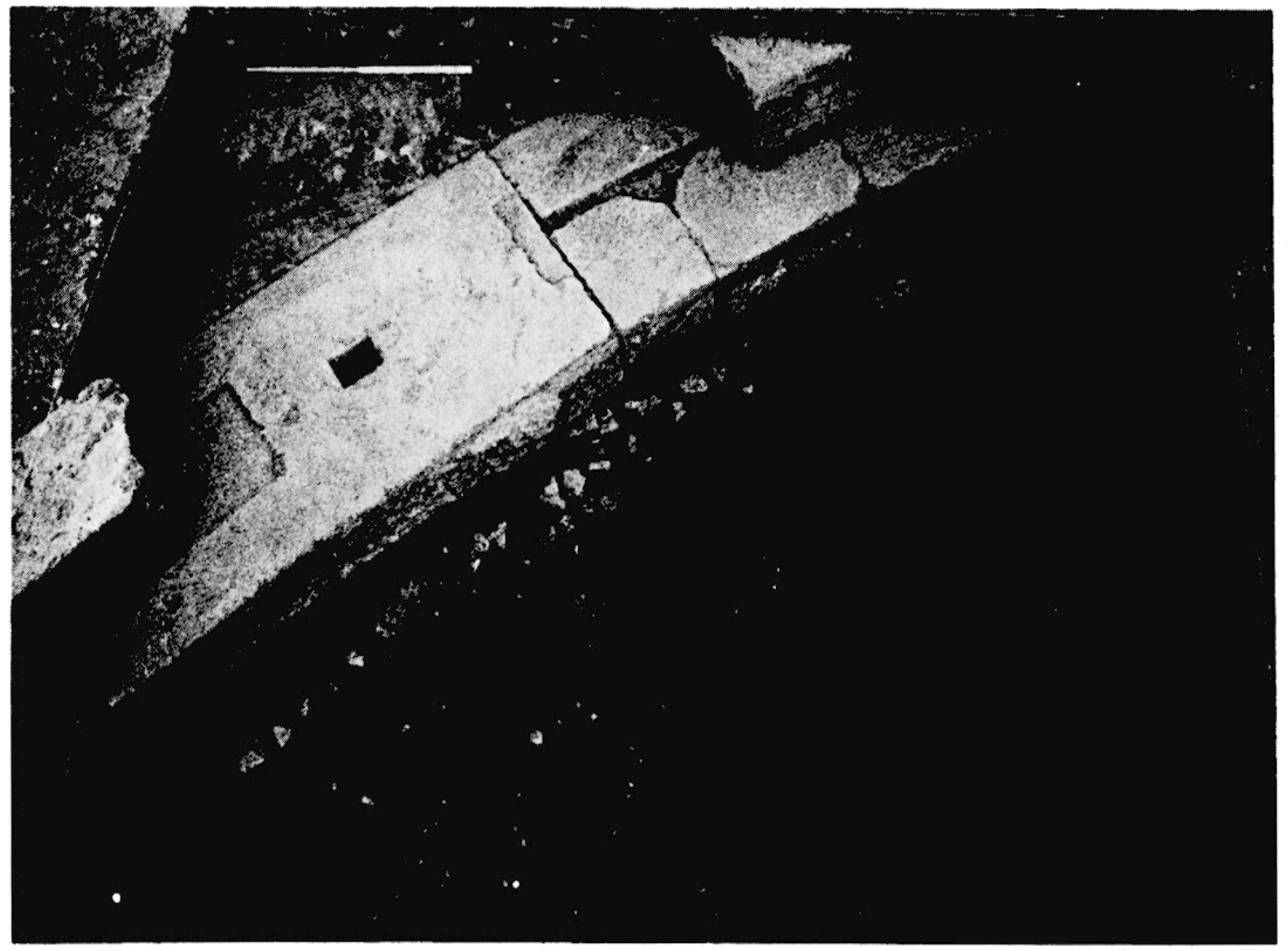

Fig. 6. - Seuil monolithe - en avant, blocage et sol bétonné (AO 77 - vers l'Ouest). 


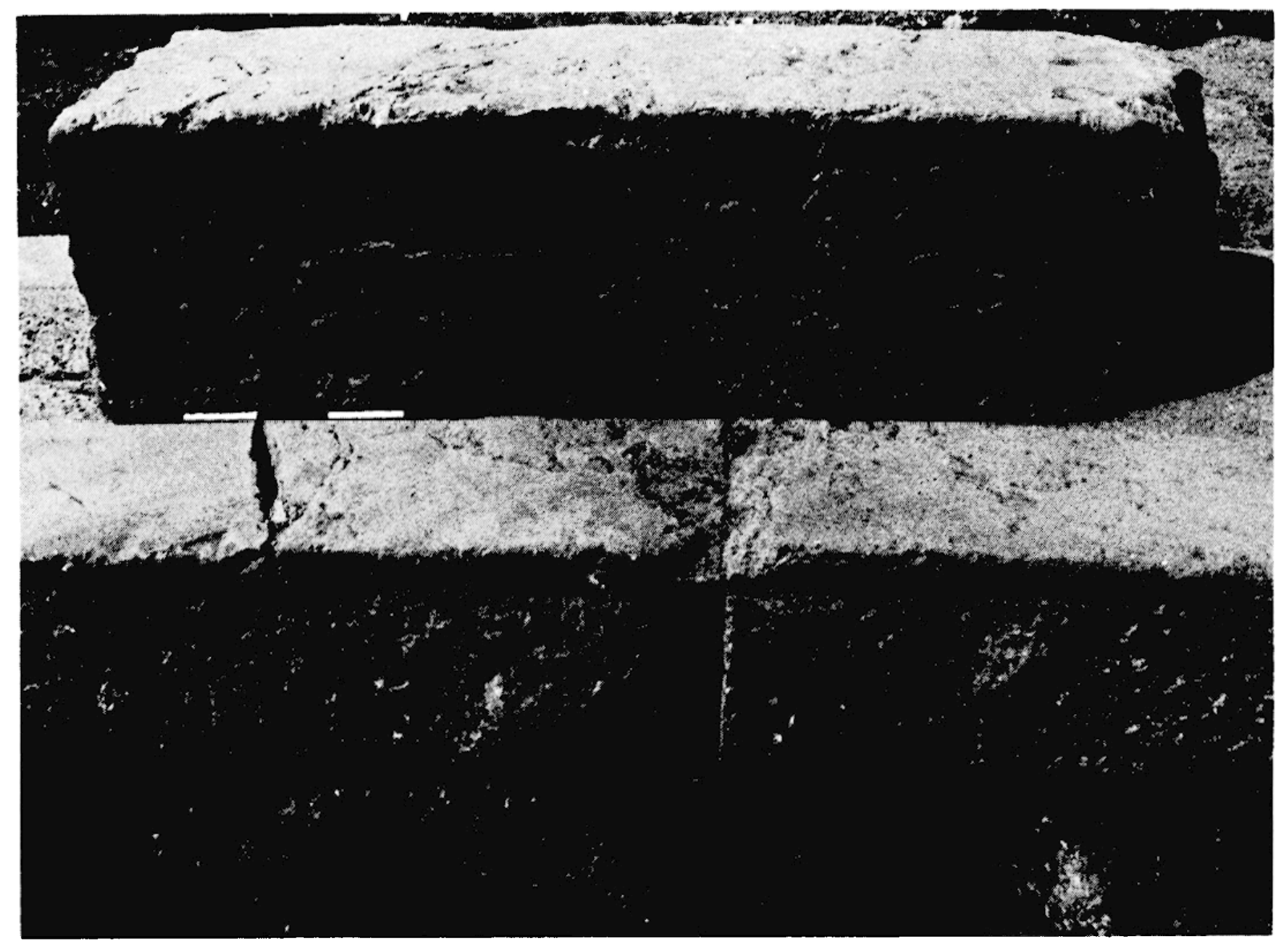

Fig. 7. - Degrés de la construction en arc outrepassé, côté intérieur (AO 77 - vers le Sud-Ouest).

Fig. 8. - Inscription sur un bloc de base honorifique romaine remployé (cliché Ph. Collet). 
Le sol bétonné en place actuellement à l'intérieur de la construction appartient, comme le prouve le travail des blocs de la face interne évoqué ci-dessus, à un second ètat. Le recreusement général de l'espace intérieur, accompagné de l'installation d'un blocage de petite pierraille et mortier compact - de même composition que le sol lui-même - contre les fondations de la construction en arc outrepassé et à l'aplomb de la krépis, s'explique grâce à la présence de quelques $\mathrm{cm}^{\mathbf{2}}$ de mortier hydraulique conservés sur le sol et sur le blocage : cette "exèdre " fut transformée en bassin, à une époque que le matériel céramique recueilli dans les remblais qui l'ont comblé - si l'on élimine les quelques tessons du géométrique à l'hellénistique qui y sont mêlés - ainsi que

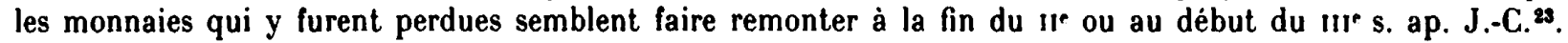

De ces mèmes remblais très èpais proviennent sept stylets ou fragments de stylets en bronze, curieusement concentrés près du dernier bloc de l'assise-banc resté en place à la limite . ord de la perturbation byzantine.

\section{LA KRÉPIS.}

La branche Ouest de cette construction en arc outrepassé vient, en A.V 76, buter contre une krépis en calcaire (fig. 9), orientée Est-Nord-Est/Ouest-Sud-Ouest, qui a été retrouvé en AM 78, A.V 75 et dans un sondage ouvert par l'Éphorie immédiatement à l'Est du café Karmoyannis : elle traverse donc le terrain d'Est en Ouest, sur une longueur de $28 \mathrm{~m}$, sans que l'on en n'ait découvert ni sa limite Ouest, en direction de la Salle Hypostyle, ni sa limite Est.

Le dernier bloc Nord de l'assise-banc, taillé en biseau, épouse par une feuillure le décrochement formé par le léger débord de l'euthyntéria par rapport au premier degré de la krépis, alors que l'espace resté libre à la jonction entre le premier degré de la construction courbe et l'euthyntéria de la krépis est comblé par un fragment de poros taillé en triangle (fig. 10).

\section{a. Les degrés.}

De la krépis n'ont èté mis au jour, en l'ètat 1987 des fouilles, que l'euthyntéria et le premier degré; ils présentent d'Ouest en Est une légère inclinaison (cote de l'euthyntéria : de $+10,55$ à $+10,42$ à l'Est; cote du premier degré : de $+10,86$ à l'Ouest à $+10,72$ à l'Est). L'euthyntéria repose à mème la terre, sans aucune fondation. La largeur de la marche est à très peu près celle du degré inférieur de la structure en arc outrepassé $(0,295 \mathrm{~m} \approx$ un pied argien). Le seul bout visible d'un bloc du premier degré (en $A M \mathrm{M}$ ) présente une anathyrose en gammau.

\section{b. Les fondations en poros.}

Au niveau du premier degré, à environ $0,60 \mathrm{~m}$ de distance vers le Nord, et parallèles à lui, ont été mis au jour de gros blocs de poros (cote moyenne $+10,80$ - visibles fig. 9), présentant parfois des refouillements, qu'il est tentant d'interpréter comme les fondations d'une colonnade ${ }^{25}$, à l'avant de laquelle on peut restituer deux degrés supplémentaires.

Nous serions ainsi en présence de la façade d'un portique qui fermait le cỏté . Nord de l'agora. La porte monumentale du terrain Tsiramanès ${ }^{20}$ semblerait le confirmer, puisqu'elle se trouve dans le mème alignement; autre preuve de l'importance topographique de cette krépis, le vaste bảtiment romain de l'angle . Vord-Ouest du terrain Karmoyannis en a, à $\mathbf{0 , 8}$ grade près, respectè scrupuleusement l'orientation.

\section{c. Le sol.}

Le sol d'origine au Sud de la krépis a entièrement disparu ${ }^{27}$; le chant des blocs de l'euthyntéria fournit cependant un indice pour en restituer le niveau, puisque la partie inférieure, au bossage grossier (sur $20 \mathrm{~cm}$ de haut en moyenne), devait être cachée aux regards.

(23) Rien de plus ancien que du Çandarli (HAyes, forme 2) et des monnaies d'Argos de 198-217 ap. J.-C.

(24) Largeur du bandeau horizontal : $3 \mathrm{~cm}$. - du bandeau vertical : $3,5 \mathrm{~cm}$.

(25) Les seuls restes de colonnes actuellement découverts sont des petits fragments retaillés conservant deux ou, au plus, trois cannelures, qui ètaient incorporés dans le blocage installé lors du remaniement en bassin à l'intérieur de la base centrale de la construction en arc outrepassé.

(26) Cf. BCH 94 (1970), p. 788-793.

(27) Le premier niveau de sol dégagé au Sud de la krépis (en A.N et AO 76) se situe à la cote $+9,50$ : il est constitué de galets de taille irrégulière pris dans une terre brun clair. La céramique qu'il livre, apparemment homogène (avec une forte proportion de Pergaménienne), semble le dater du ier $\mathrm{s}$. ap. J.-C. 


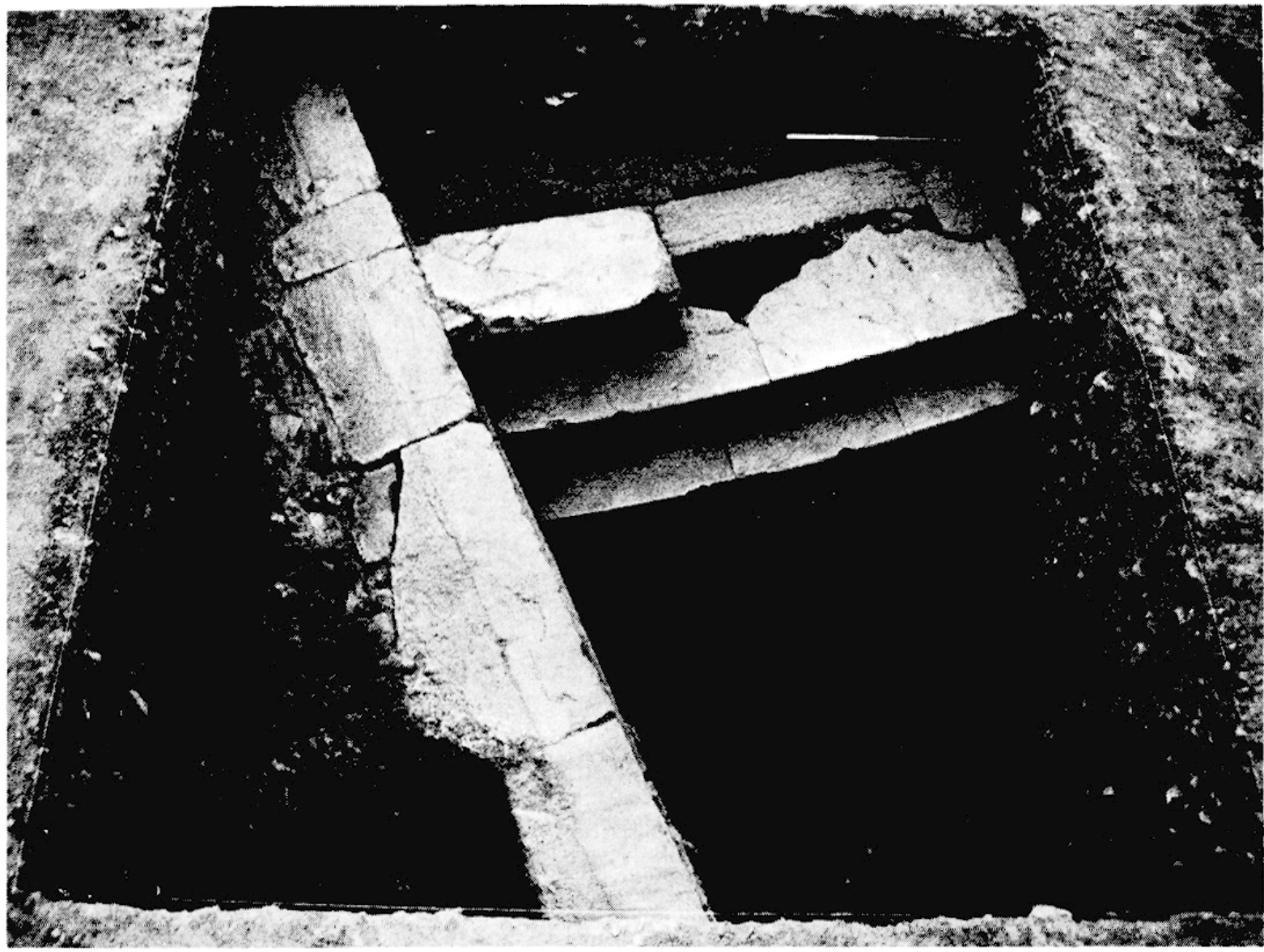

Fig. 9. - Jonction entre la construction en arc outrepassé et la krépis (AN 76 - vers l'Est).

\section{Problemes de chronologie.}

Les indices chronologiques en rapport avec la krépis et la construction en arc outrepassé sont quasiment inexistants.

a. Chronologie relative.

Un examen détaillé du mode de jonction des blocs de l'un et de l'autre bâtiments (feuillure de l'assise-banc, ravalement de l'euthyntéria sur toute sa hauteur seulement à son point de jonction avec le degré supérieur de la construction courbe [en AN 76], taille en pointe irrégulière, destinée à être cachée, de l'extrémité Nord du degré inférieur de la construction courbe, qui s'engage de $15 \mathrm{~cm}$ sous l'euthyntéria de la krépis) semble prouver que la structure en arc outrepassé a èté installée en second lieu contre la krépis d'un portique déjà en place. La similarité du travail des pierres et des dimensions, la conception même de l'ensemble concordent cependant à suggérer que le laps de temps séparant les deux constructions ne fut pas très long.

\section{b. Chronologie absolue.}

Les sols, intérieur comme extérieur, ayant entièrement disparu, les éléments de datation directement en rapport avec la construction ou l'utilisation de ces deux bâtiments font, en l'état actuel des fouilles, cruellement défaut.

- Examen des blocs.

Les dimensions moyennes des bandeaux d'anathyrose visibles sur quelques-uns des blocs sont celles qui apparaissent fin $v^{e} \mathbf{s}$, et sont adoptées dans les constructions classiques ${ }^{28}$.

(28) Cf. R. MArTin, Manuel d'architecture grecque, I (1965), p. 196. 


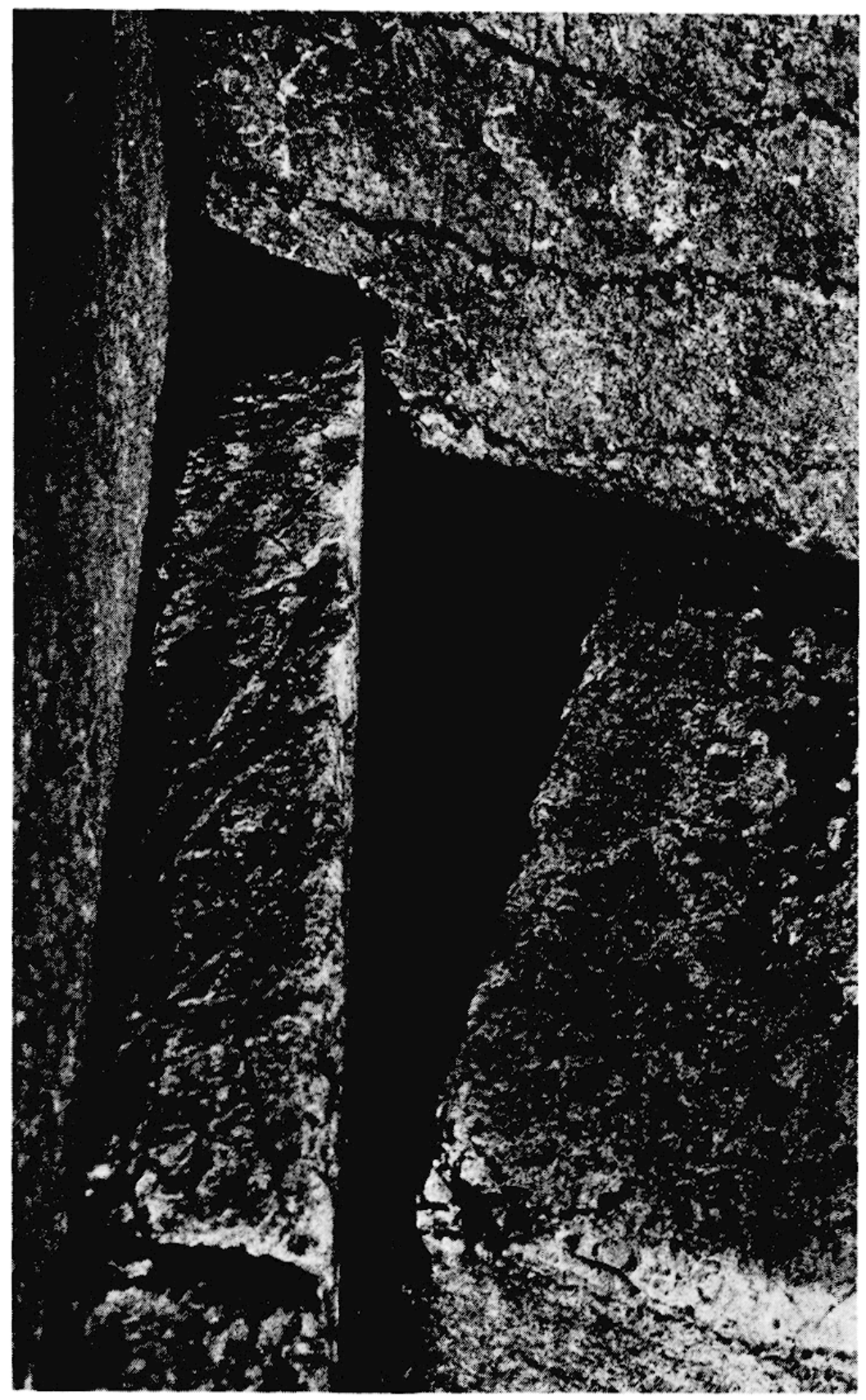

Fig. 10. - Détail de la jonction des deux constructions (AN 76 - vers l'Est).

De mème le seul scellement actuellement visible sur la construction en arc outrepassé est un goujon du type de ceux dont l'emploi s'est généralisé au ve s. av. J.-C.20.

- Données stratigraphiques.

Le seul sondage ouvert en 1987 au Nord des fondations en poros attribuées à la colonnade (moitié Sud de AL 78) a livré, dans une couche de terre brun orangé fine et homogène, outre une petite fosse paléochrétienne, une grosse quantité de tessons géométriques et archaĩques, mélés de quelques tessons classiques. Un petit amas de pierraille contenait, avec quelques ossements animaux, des tessons exclusivement géométriques.

(29) Ibid., p. 283, fig. 126, 131, 134. 
En attendant des prochaines fouilles les indices chronologiques fiables et précis indispensables, il semble du moins, en l'état actuel de la recherche, que le type de construction, le travail des blocs, les détails techniques mentionnés ci-dessus permettent de suggérer pour ces deux bâtiments une date dans l'époque classique, sans qu'il soit encore possible de choisir entre le $v^{e}$ et le ive $s$. (plus vraisemblable cependant, si l'on en croit la date d'apparition de cette forme dans les théâtres, pour la construction en arc outrepassé).

La campagne de fouilles 1988 devra en outre s'efforcer de faire la jonction entre la krépis attribuée ici au portique Nord de l'agora et le grand bâtiment romain dégagé en 1972 à l'angle Nord-Ouest du terrain Karmoyannis ${ }^{30}$.

II. Une fouille supplémentaire a été menée, sur demande de l'Éphorie des Antiquités de Nauplie ${ }^{31}$, avec une équipe d'ouvriers, du 3 au 20 aout 1987, dans le but de nettoyer et d'étendre vers le Nord la fosse septique des anciennes toilettes situées près de l'angle Sud-Est du café, contre l'actuelle clôture du site. Afin de permettre une fouille cohérente de cette partie du terrain, les limites de la future fosse septique ont été intégrées au quadrillage du secteur, la zone fouillée constituant un carré de $4 \mathrm{~m}$ de cóté (les $2 \mathrm{~m}$ Ouest de AQ 74 et les $2 \mathrm{~m}$ Est de AQ 73).

1. Le nettoyage de l'ancienne fosse septique (jusqu'à la cote $+10,45$ ) a livré un certain nombre de blocs architecturaux en poros (dont un fragment d'architrave avec une regula où sont conservées deux gouttes), un fragment de meule en basalte, un fragment de base de colonnette cannelée en terre cuite, et quelques fragments d'un grand pithos géométrique.

2. Au Nord de cette fosse, on a mis au jour :

a. un muret probablement moderne (cote $+11,75)$, orienté Sud-Ouest/Nord-Est, dont le démontage a livré un beau fragment de chapiteau corinthien en marbre;

b. deux squelettes de la grande nécropole récente qui couvre l'agora (cotes $+10,89$ et $+10,86$ ), dont le fond de la fosse reposait sur

c. un sol très compact, formé d'un amalgame de gravier de taille irrégulière et de nombreux fragments de tuiles très usés pris dans une terre sableuse brun clair (cote $+10,75)$, posè sur une couche sableuse mèlée de très abondant gravier.

3. Ce sol recouvrait :

a. dans la partie Nord du sondage, à la cote $+10,45$, une base construite en petits blocs de poros (probablement des blocs de remploi retaillés) et pierraille, liaisonnés au mortier, qui repose sur un socle de gros galets et mortier; l'ensemble est daté par la céramique environ des nue-Ive s. ap. J.-C. Au sommet de cette base, dans sa partie médiane, a été dégagée une couche agglomérée de galets, pierraille et fragments de tuiles qui pourrait constituer les restes d'un sol (?).

b. Cette base est collée contre la face Nord d'un bloc de poros, allongé Nord-Ouest/Sud-Est, de 1,20 m de long sur $0,65 \mathrm{~m}$ de large (cote $+10,28$ ). Il repose, pour sa moitié Ouest, sur une couche sableuse brun rougeâtre, mêlée d'abondant gravier, presque stérile, que l'on retrouve dans l'ensemble du sondage à la cote $+9,95$, et pour sa moitié Est, sur un autre bloc de poros (cote $+9,97)$, de $1,35 \mathrm{~m}$ de long sur $0,61 \mathrm{~m}$ de large, orienté perpendiculairement. On a mis au jour, jointif de ce premier bloc d'une assise inférieure, un second bloc de poros, de mème taille et même orientation (fig. 11).

Ces trois blocs sont les vestiges des fondations du mur Nord de la Salle Hypostyle ${ }^{32}$, composées pour compenser la pente du terrain d'une assise à l'Ouest et de deux assises à l'Est. Le prolongement vers l'Ouest de ces fondations, ainsi que, semble-t-il, leur retour vers le Sud à la jonction avec le mur de façade Est du bâtiment $^{33}$ ont disparu dans des tranchées de récupération livrant un matériel céramique qui, en première analyse, semble autoriser à dater la destruction de la Salle Hypostyle du IV s. ap. J.-C. ${ }^{\mathfrak{4}}$.

(30) Cf. $B C H 97$ (1973), p. 483-489.

(31) Le TAПA ayant décidé de réhabiliter le café Karmoyannis, il fallait creuser l'emplacement nécessaire à une fosse septique.

(32) Le bâtiment doit être publié prochainement par J.-F. Bommelaer et J. des Courtils dans un volume des EtPélop.

(33) Sur la disparition de l'angle Nord-Est, cf. G. Roux, BCH 77 (1953), p. 244.

(34) C'est la date avancée par les fouilleurs de l'agora pour la récupération des blocs du mur Est (cf. $B C H$ 100 [1976], p. 754), et que proposait déjà G. Roux pour la destruction du bâtiment (cf. BCH 77 [1953], p. 246). 


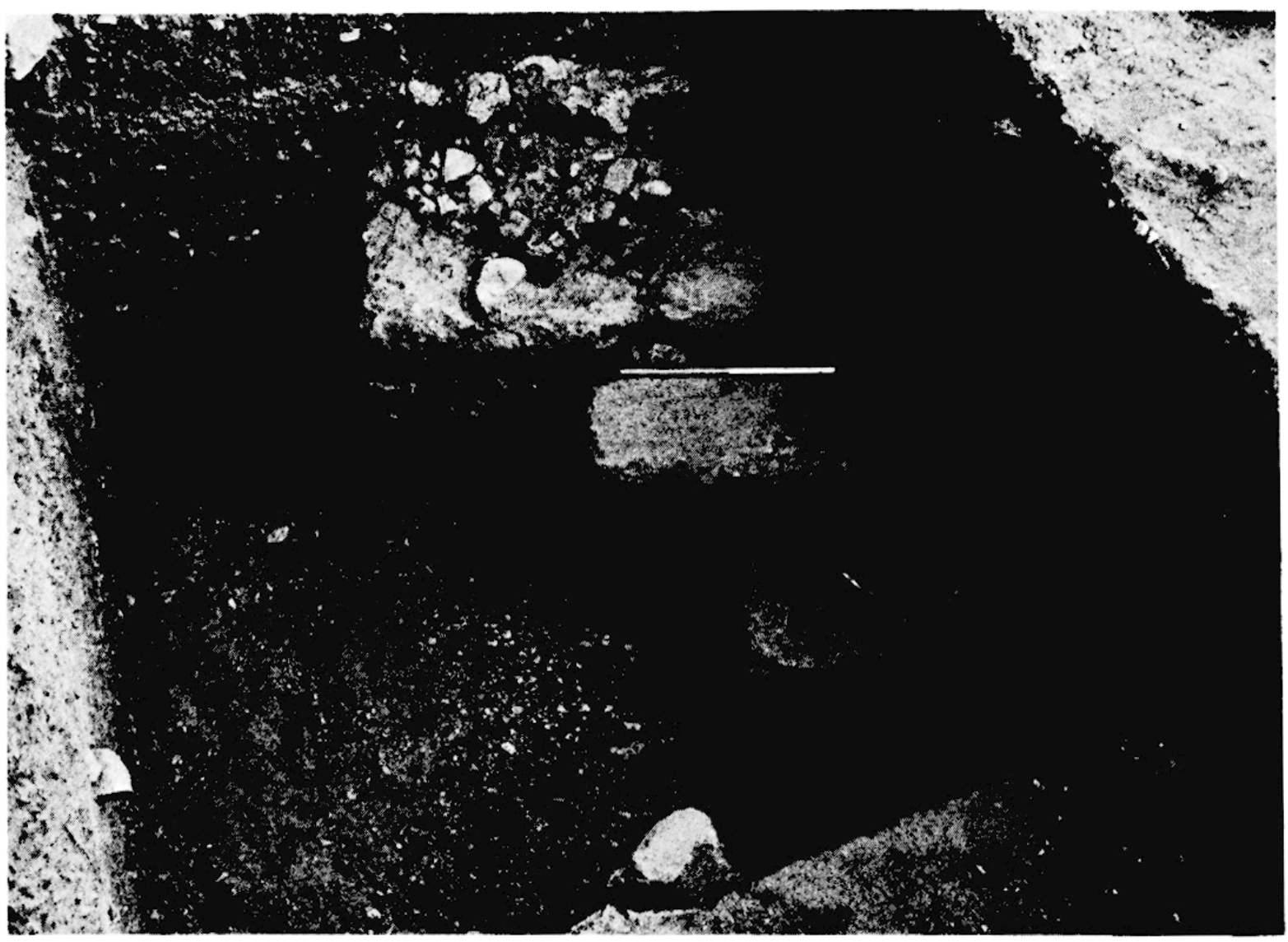

Fig. 11. - Base romaine et restes des fondations du mur Nord de la Salle Hypostyle (AQ 73-74 - vers le .Nord).

De part et d'autre de la tranchée de récupération, dont le fond est constitué par une surface de terre très tassée dans laquelle sont agglomérés des déchets de poros et du petit gravier, on trouve à partir de la cote $+10,23$ une couche de terre brun roux très fine et très compacte, livrant peu de matériel - en majorité très fragmentaire -, uniquement archaïque, en particulier une oenochoé à vernis noir écrasée en place, de la seconde moitié du vr s., et quelques rares tessons géométriques.

La fouille a été arrêtée à la cote $+9,47$, dans la couche sableuse à gravier sur laquelle reposent les fondations du mur, sans avoir atteint le sol vierge. Il serait intéressant, pour la publication à venir du bâtiment, de poursuivre l'exploration des couches apparemment archaiques encore en place aux abords immédiats, intérieurs comme extérieurs, du mur Nord de la Salle Hypostyle.

Pour des raisons évidentes d'intérêt archéologique, et des problèmes de présentation future et d'aménagement de l'ensemble du site de l'agora, l'installation d'une fosse septique à cet emplacement n'est plus envisagée ${ }^{35}$.

(35) Une troisième fouille a donc dù être menée (du 12 au 30 octobre 1987), en collaboration avec K. Barakari, épimélète des Antiquités à Nauplie, avec une équipe de l'Éphorie. Les résultats de ce dernier sondage - implanté dans le prolongement du mur Est du café Karmoyannis, à $1 \mathrm{~m}$ au Sud de la partie arrière agrandie sur une dalle de béton coulée en janvier 1987 - qui excluaient à nouveau l'installation de la fosse septique, seront exposés dans un périodique grec. 


\title{
3. - Temple et thermes A
}

\author{
par Pierre Aupert
}

InTRODUCTION.

La campagne, qui s'est déroulee en octobre, a bénéficié de l'assistance de Judith Hoogendijk et Michael Frel, étudiants à Amsterdam, Marion Wittgrefe, étudiante à Bonn, Isabelle Favarel-Garrigue de Bordeaux III et Catherine Petit de Toulouse-le-Mirail.

Dans la partie thermale proprement dite, l'intervention s'est limitée à la mise en évidence d'une porte, jusqu'alors inaperçue, qui met en communication les latrines $\mathrm{H}$ et la stoa $\mathrm{N}$. Cette découverte conforte l'hypothèse de la suppression de la colonnade dans toute la zone $E$ du båtiment lors de l'édification de l'état II (thermes), de son remplacement par un mur plein et de la création, par là, d'un couloir de service au niveau public. Une canalisation forcée, nouvelle pièce du réseau d'alimentation, échancre le panneau $O$ de cette porte. L'essentiel de la campagne a porté en B4 et en avant de la façade. /On se reporlera au plan du BCH 111 (1987), p. $598]$.

\section{Un temple antérieur au Sérapieion-Asclépieion de l'étal I?}

En B4, l'installation d'une masse de béton et de tirants destinés à retenir le mur $\mathbf{N}$ de la salle, dangereusement incliné vers la rue du Théătre, a entraîné l'extension de la fouille de 1985 (cf. BCH 110, p. 769), sur une largeur de $4,90 \mathrm{~m}$ à partir de ce mur. L'enlèvement de la mosaique a permis de dater cette dernière du Ive $s$. de n.è. Au-dessous, un remblai du jer $\mathbf{s}$. de n.è. et des sols de mortier liés sans doute à la construction de l'état I, noyaient une fondation Est-Ouest (fig. 12) en blocs de calcaire et de póros, dont les prolongements et les retours vers le Sud avaient déjà été mis au jour, sous A2 et la stoa $\mathbf{N}$.

Pour l'instant la datation demeure difficile : elle est à placer sûrement au IIe $\mathbf{s}$. av. n.è. au plus tôt, mais peut être portée éventuellement au jer s. de n.è.

L'importance de cette fondation, longue de $17 \mathrm{~m}$, sa vraisemblable orientation à l'Est et sa surélévation par rapport aux constructions immédiatement voisines à l'Est, incitent à l'attribuer à un temple, prédécesseur du grand Sérapieion-Asclépieion qui l'a fait disparaitre.

Le remblai a fourni un fragment de statuette en terre cuite (fig. 13), qui évoque celles des desservants du culte isiaque trouvées à Amathonte (P. AUPERT, $B C H$ 105, p. 377-378, fig. 8; A. QueYrel, Amathonle IV [1988], nos 347-376). Il est regrettable que l'exiguïté de cette pièce ne permette pas une identification plus assurée, qui eût fourni un indice supplémentaire pour celle du bâtiment lui-même.

\section{En fagade des thermes.}

a. L'aqueduc de AO 59.61.

La construction qui s'accole à l'extrémité Sud de la façade (fig. 14) est très vraisemblablement à interpréter comme un départ d'aqueduc. Elle se présente, en effet, comme un alignement de trois piliers, contre lesquels s'est amoncelé un très épais dépôt calcaire et les conduites de trop-plein des stoas Est et Sud des thermes se dirigent précisément vers cet endroit.

Le sol correspondant à ces piliers $(13,34)$, curieusement inférieur à la sortie de fondation de la façade des thermes, n'a guère été fouillé et paraît dater du rve siècle. Par ailleurs, le lestaceum de ces piliers, avec un coefficient de densité de briques de 1,32 , est identique à celui de l'aqueduc qui longe le mur Sud de la salle hypostyle (1,3), lui-mème daté de la même époque (ff. $B C H 97$ [1973], p. 482 et 105 [1981], p. 904). Enfin, on sait que l'extension c.2 de l'aqueduc Sud, dans la parodos du théâtre, date de la première moitié de ce siècle. L'ensemble pourrait donc bien constituer un système cohérent, destiné à alimenter les thermes, puis les installations hydrauliques de l'agora.

A la fin du IVe s., on a construit, à partir de 14,26, un mur 7 entre les piliers Est. La couche de destruction, mélée de concrétions calcaires, de charbons et d'ossements animaux, est datée de 585 en raison de la céramique slave qu'elle recèle. Après cette destruction, un mur 5 de direction Nord-Sud et fait de remplois, s'est accolé à la face Nord du pilier central. La raison de ces constructions n'est pas apparue à l'intérieur des limites du sondage. 


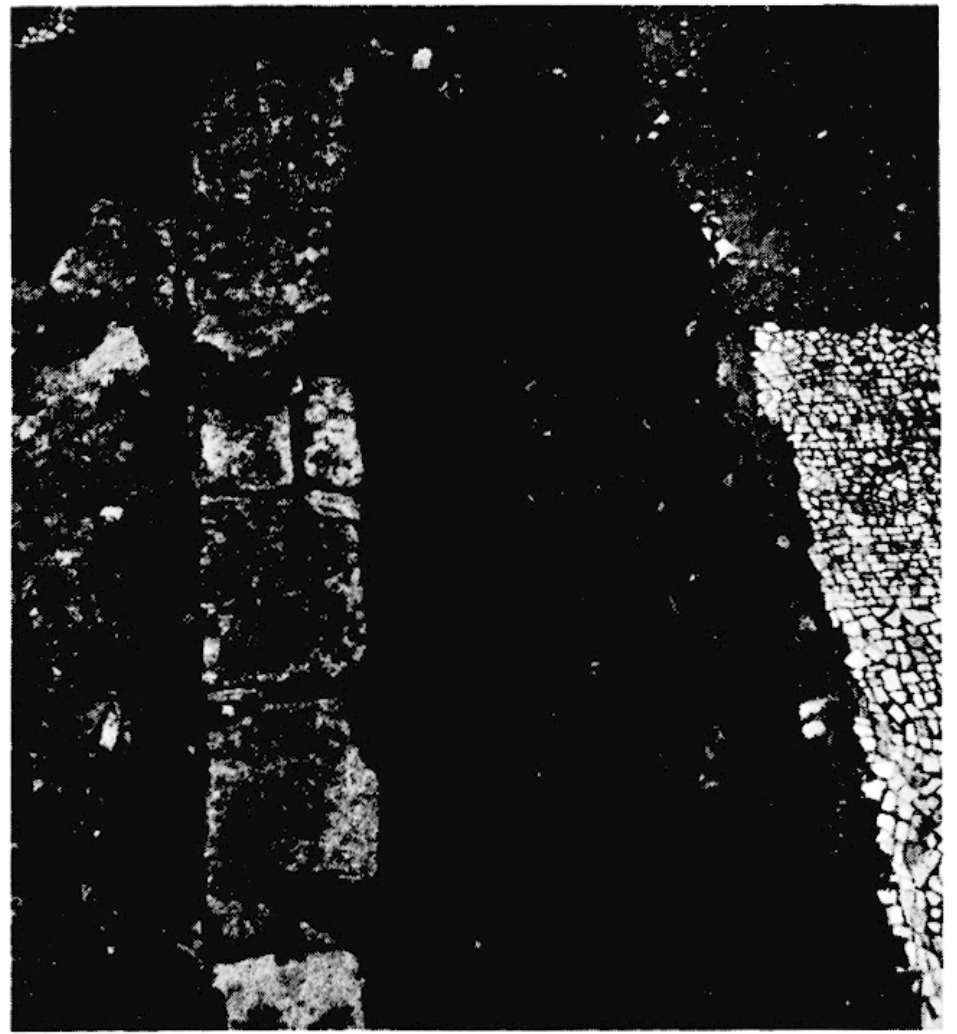

Fig. 12. - Salle B4; fondation Est-Ouest vue de l'Ouest.

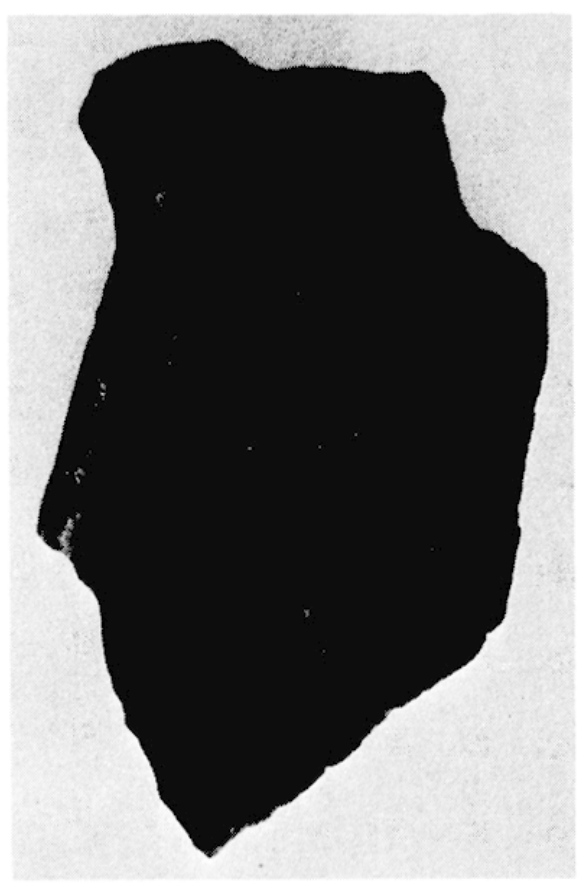

Fig. 13. - Figurine en terre cuite.

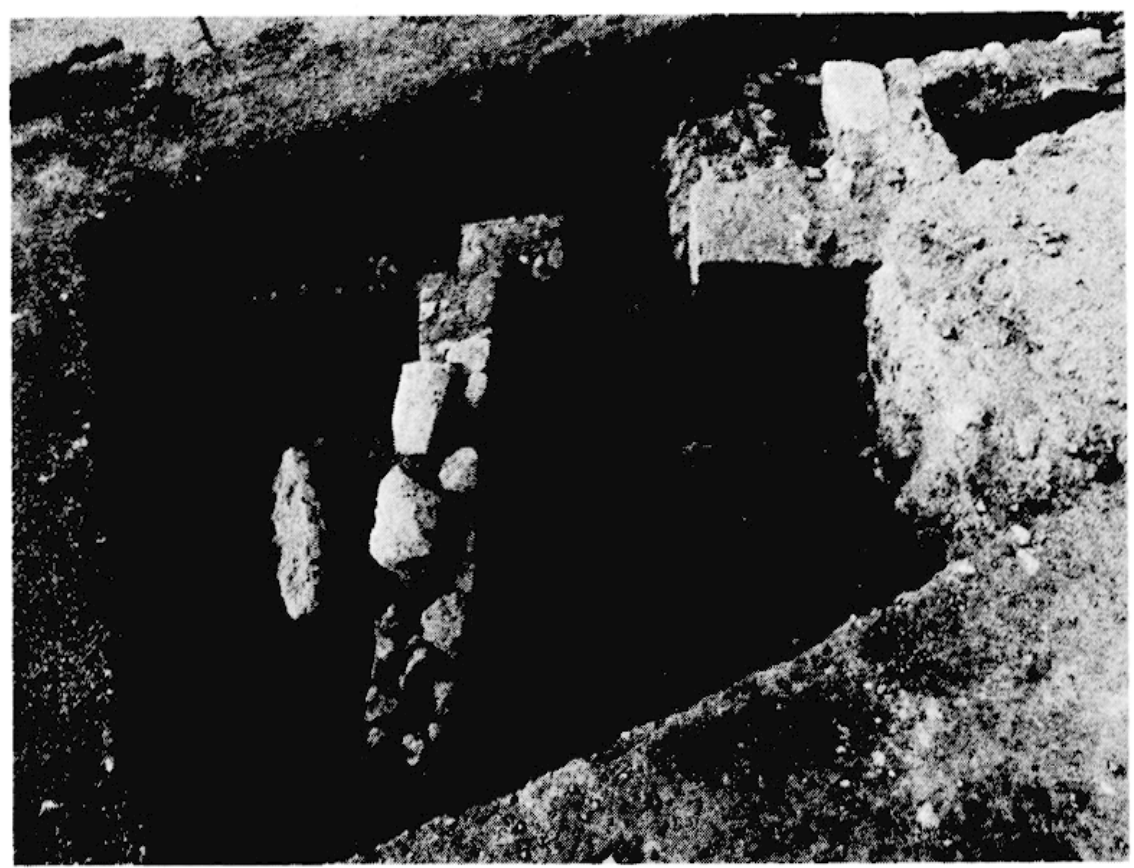

Fig. 14. - Sondage AO 59.61, vu du Nord. Façade des thermes à dr. Mur récent (post vie s.) au premier plan. 
b. La rampe.

La rampe est maintenant entièrement dégagée (fig. 15). La limite Est en a été trouvée, à l'Est des conduites modernes, sous le trottoir de la rue de Tripoli, en AM 66 (fig. 16) : seuil en calcaire, en retrait par rapport à une sorte d'ante en maçonnerie munie d'une conduite tubulaire verticale, noyée dans le caementicium.

Le hall Ll possède une série de sols en terre battue, dont les derniers, I' et I. vers 13, 10/13,05 et 13,00, datent du vie s. Au-dessous, un sol II date du rve ou du ve s. Le sol primitif de la salle, sur lequel sont tombés des fragments de placage en marbre, parait être un sol IV $( \pm 12,72)$. La couche sous-jacente ne renferme que de la sigillée ancienne. Il n'est du reste pas exclu que le mur $\mathbf{N}$ de la salle au moins ait comportè deux états, le premier lié au sol IV et le second au sol II. Quant à la conduite c.52, que l'on voit au Sud de Ll (fig. 15), elle est antérieure à l'installation de L2, qui l'a coupée.

En K1, le sol I $(13,83)$, surélevé en relation avec l'installation du mur $\mathrm{N}$ et du seuil, date du ive siècle. Le sol $11(13,45)$ est porté par une couche hellénistique. Bien qu'il corresponde aux sorties de fondation, on ne peut donc rien conclure en ce qui concerne la date de la salle et de la rampe qu'elle supporte.

En K2, on a déplacé l'une des masses de maçonnerie de la voute effondrée, pour dégager les sols. Ils sont plus élevés que leurs correspondants en $\mathrm{Kl}$ (14, 24 pour I et 13, 72 pour II) et le sol I parait dater du ve $s$.

Des sondages sous le sol II de Kl, répartis au Nord de l'axe du båtiment, n'ont révélé aucune fondation d'escalier antérieur.

\section{Dalation.}

L'appareil de la rampe offre des disparates dont il faut tenir compte. En comparaison des murs Nord-Sud des chambres K1-K2, on constate que le mur d'échiffre Nord est dépourvu de bipedales, que ses zones en incertum sont de factures plus relâchée et son coefficient de densité de brique, pour les zones en lestaceum, est de 1,35 , contre 1,50 pour l'intérieur des chambres. Les couches extérieures qu'il parait bien avoir percées datent de la fin du IIre ou du Ive s. : tous ces indices le rendraient donc contemporain de l'aqueduc de AO 60.61 .

Or, ce mur est mal relié aux refends qui constituent les parois Nord-Sud des chambres et celles-ci possèdent presque les mèmes caractéristiques (densité $=1,50$, arases de bipedales) que les murs des salles A2 et A3 $(\mathrm{d}=1,61$, arases de briques de $0,62 / 0,64 \mathrm{~m})$, attribuées au règne de Gordien III.

Tout se passe donc comme si, sous Gordien III, on avait construit, en mème temps que ces salles, la rampe (de R1 à R4), puis, vers 300-350, les murs de L1-L2, ainsi que les échiffres $N$ et $S$, avec des sols I rehaussés, en relation avec les seuils $\mathrm{N}$.

La couche de destruction, où l'on a achevé la fouille de la tombe découverte l'an dernier en AM 65 et dépourvue de matériel, a fourni à nouveau de la céramique slave, mais aussi, en relation avec un bassin tardif maçonné sur la rampe, quelques tessons glaçurés. Un fragment d'une vasque en marbre de grandes dimensions reposait en pleine couche, à la hauteur de L2 (fig. 17). Des fragments de fut de colonne et de chapiteau, ainsi qu'une marche d'escalier proviennent de K2.

\section{Les abords de la rampe.}

Des installations tardives sont venues s'adosser au mur d'échiffre Nord de la rampe et, notamment, une pièce dallée de plaques de terre cuite, munie d'une citerne en AK 63.64 (fig. 18).

Entre cette citerne et la rampe s'étaient accumulés, lors de la phase d'abandon du site, des éléments divers, dont un bassin en calcaire et un pan de mur d'échiffre. Au-dessous, une cavité s'ouvre dans le dallage. Son exploration a été entamée et a fourni des fragments d'un grand pithos en terre cuite, mais son origine n'a pas pu encore ètre éclaircie.

En AJ 62, la couche de destruction recélait un fut de colonne en marbre de petit module (fig. 19) et a été percée, contre la façade du bâtiment, par une de ces tombes récentes qui parsèment la région et sont uniformément dépourvues de matériel autre que des bagues indatables. Pour la première fois, ici, l'ensevelissement du défunt, en position contractée, parait avoir été complété par le dépôt d'un vase incomplet (Aig. 20). Mais ce vase est d'une fabrique qui me parait encore inconnue et sa datation reste en suspens.

En AL 65, on a trouvé, dans la même couche, un fragment de sculpture : un support, sans doute, imitant le rocher, et des fragments d'architecture en pôros qui peuvent provenir de la façade de $\mathrm{Ll}$.

Le puits de AN 65 a été désobstrué. Il est vide sur une profondeur de $2,40 \mathrm{~m}$ et les premiers tessons que l'on y recueille datent du vie $\mathbf{s}$. Il a donc dú être abandonné, comme toute la zone, en 585 . 

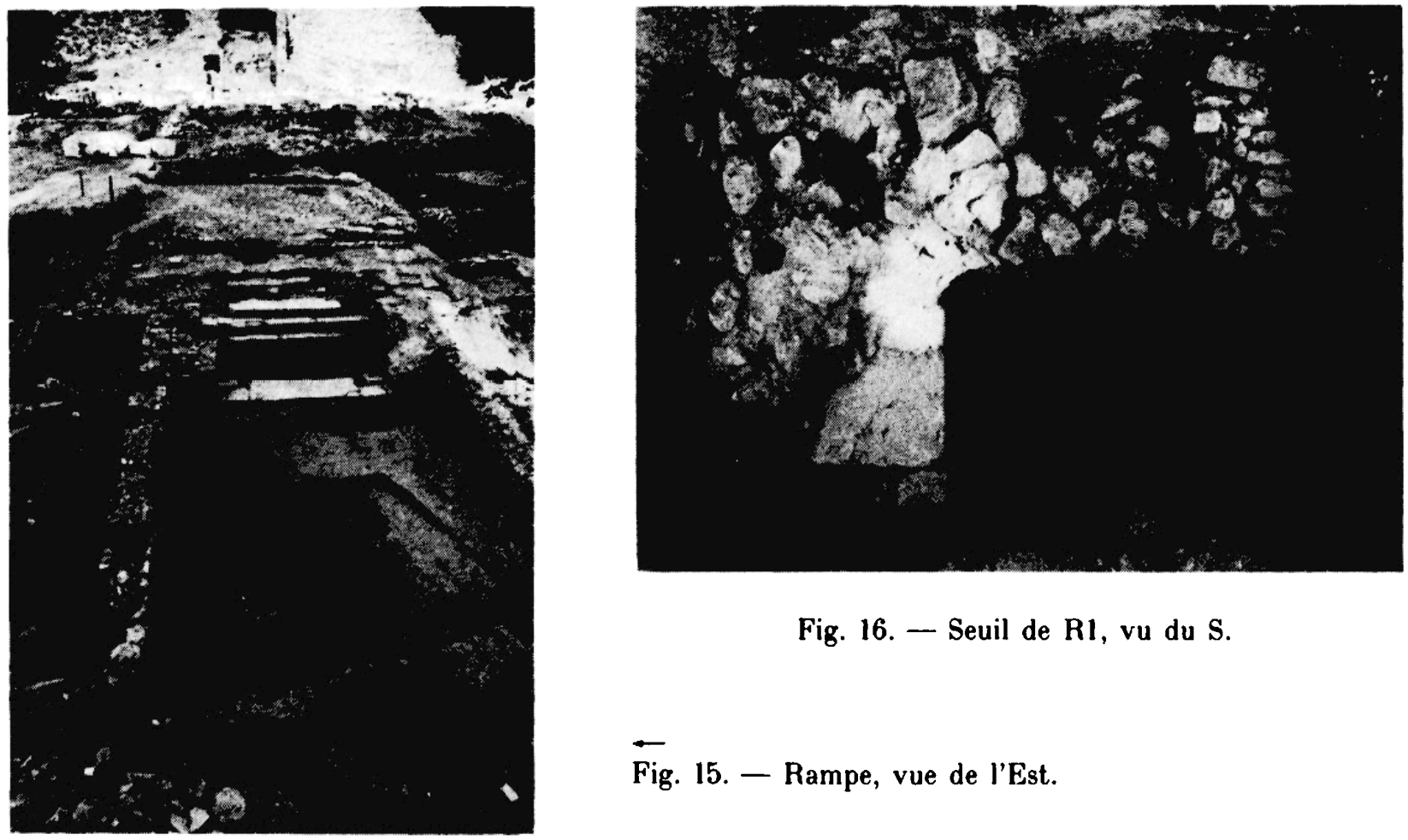

Fig. 16. - Seuil de R1, vu du S.

Fig. 15. - Rampe, vue de l'Est.

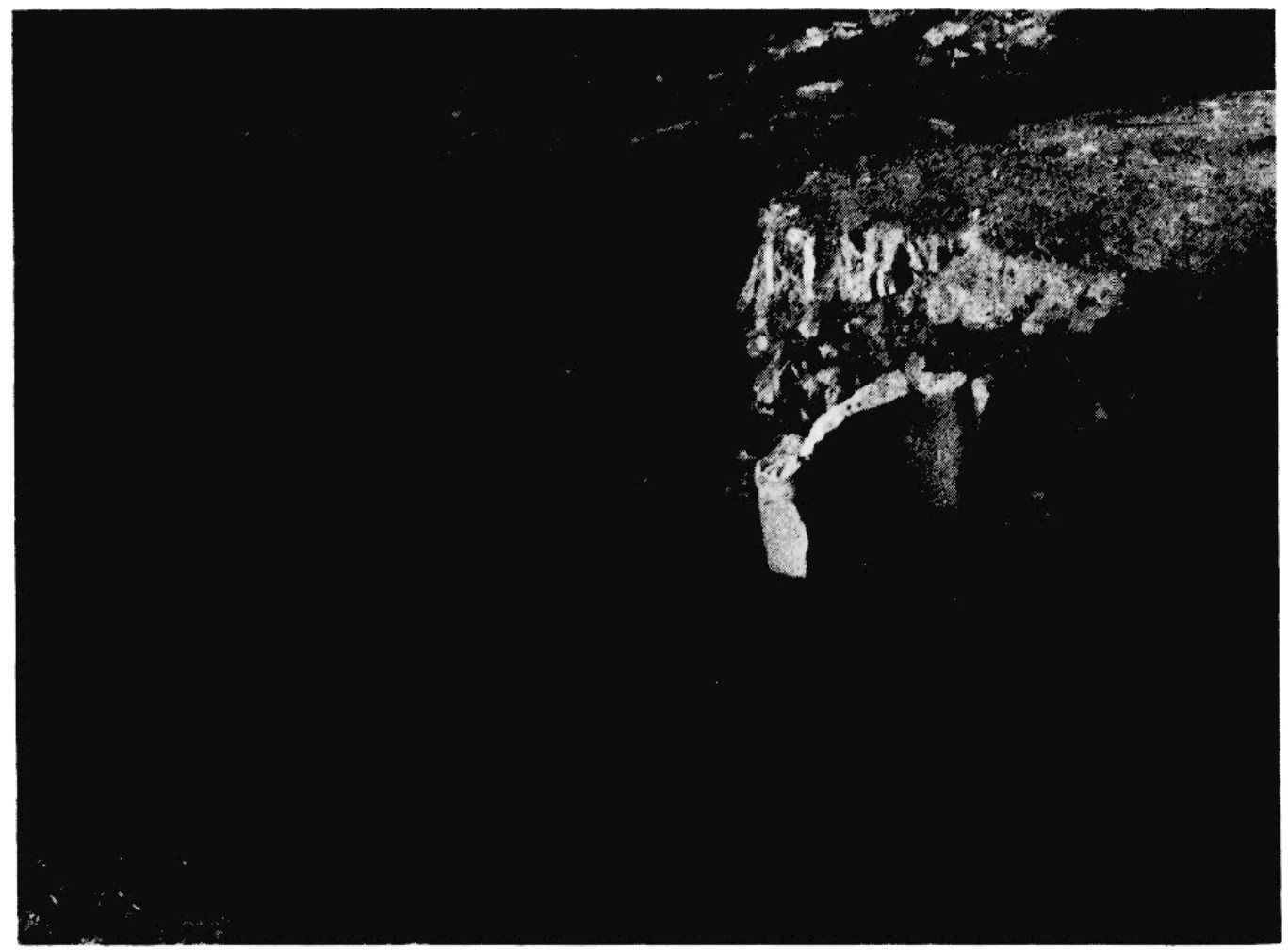

Fig. 17. - Vasque dans la couche de destruction, du Nord-Est. 


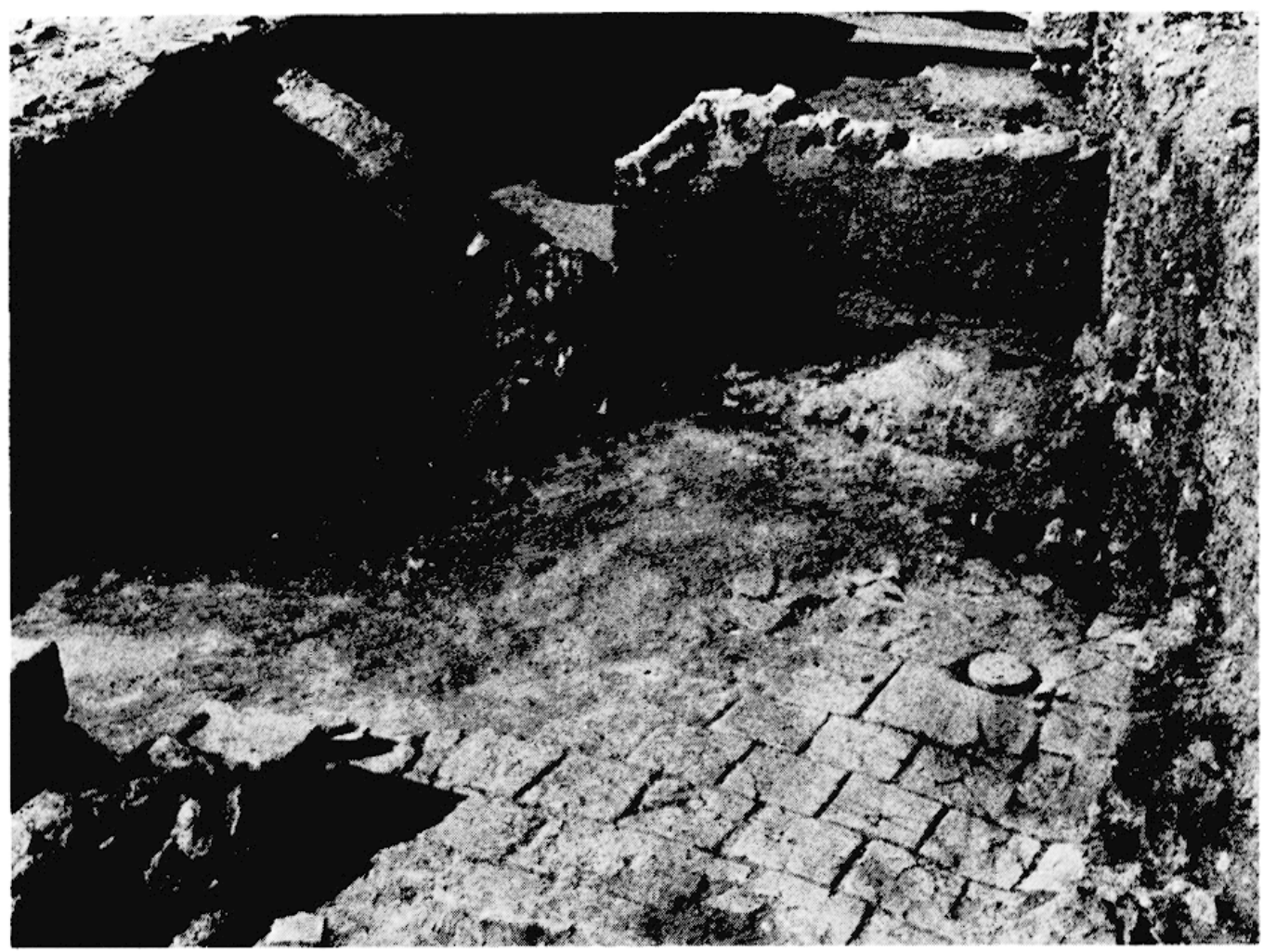

Fig. 18. - Pièce dallée, citerne et maçonnerie effondrée au Nord de la rampe.

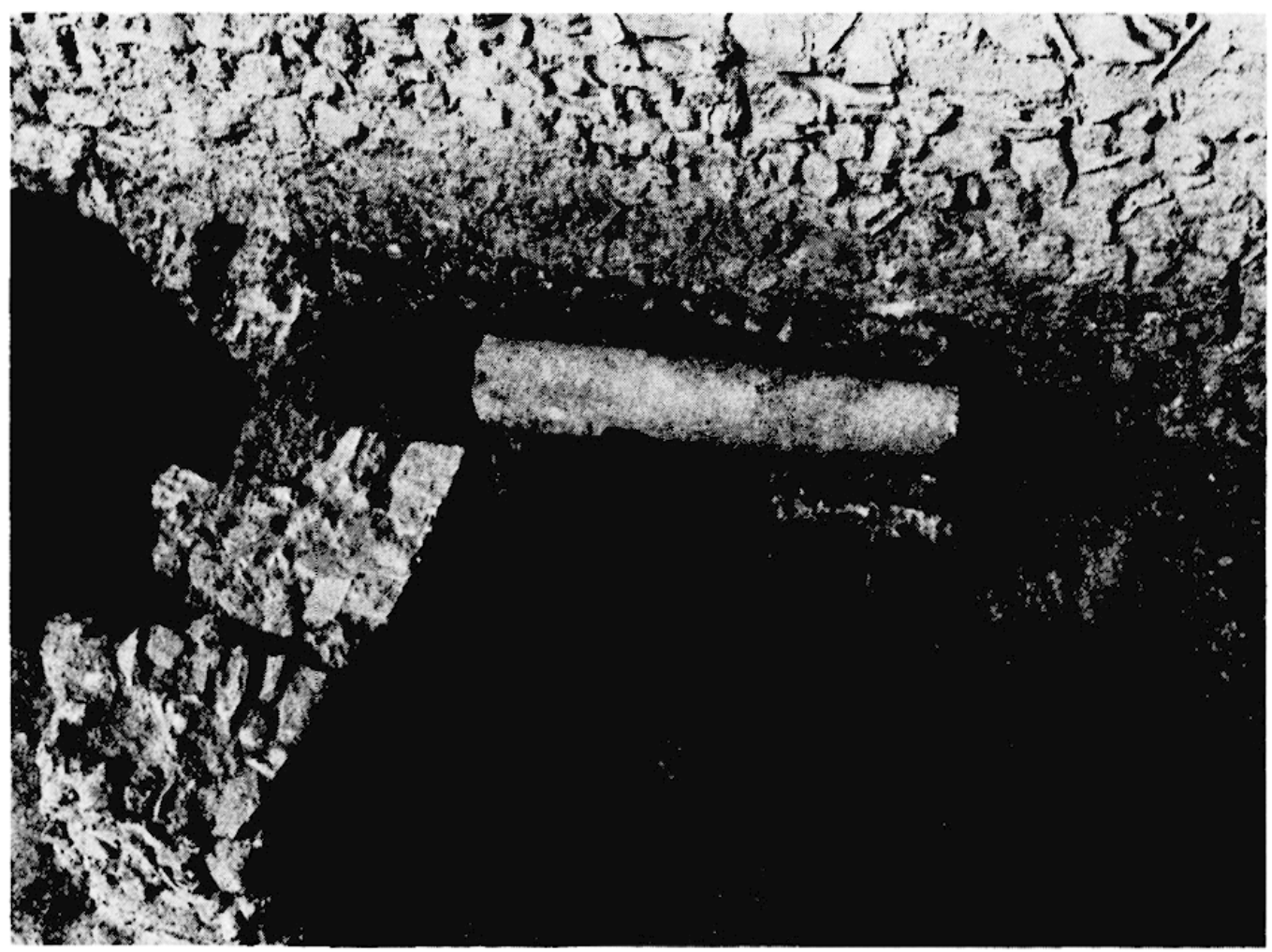

Fig. 19. - AJ 62. Au fond, la façade des thermes. 


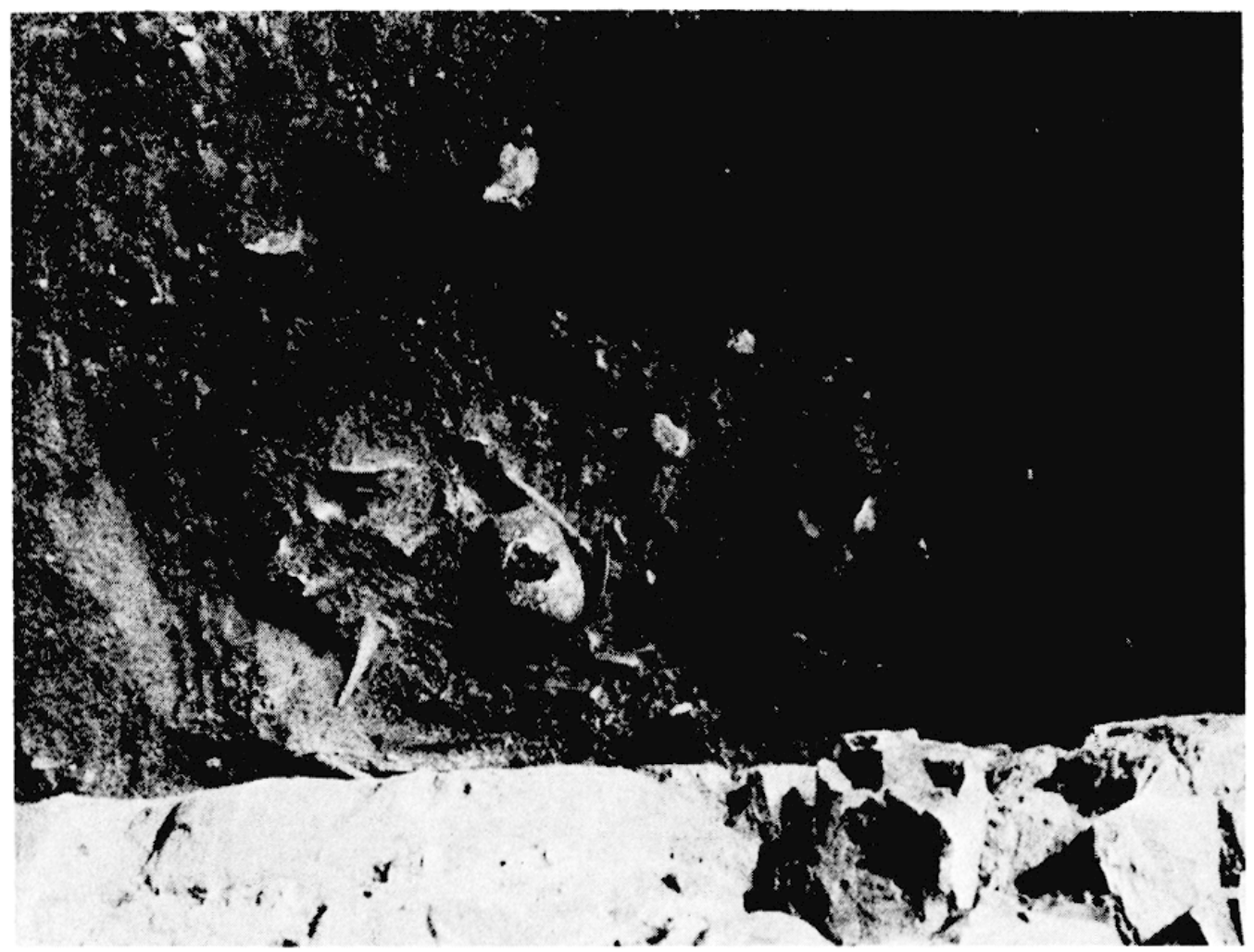

Fig. 20. - Tombe de AJ 62.

\section{Trouvailles diverses.}

Parmi les objets recueillis, mentionnons une lampe nord-africaine inscrite $\mathrm{X}$ et $\mathrm{O}$ (?), de nombreuses lampes signées, dont l'une, de type Bovon 232/233, sur l'anneau du pied et une autre, peut-ètre, sur le rebord du disque. Enfin, un objet en pierre polie et peinte ressemble à une arme préhistorique.

\section{Conclusion.}

L'extension exacte de la rampe est maintenant connue. Mais les résultats les plus intéressants de cette campagne, sont l'un positif, l'autre négatif. Après l'identification, ici ou là sur le site, d'éléments attribuables à un culte égyptien antérieur au grand Sérapieion-Asclépieion, il est enfin satisfaisant de pouvoir relier ce culte, fût-ce au titre d'hypothèse de travail, avec une construction.

Par ailleurs, ni sur la façade du temple-thermes, maintenant largement dégagée, ni sous la rampe des IIre et Ive s., nous n'avons pu découvrir trace d'un accès antérieur : qu'un bátiment de cette ampleur n'ait pas ouvert sur l'agora dès son origine et qu'il ait fallu attendre pour cela qu'il fut devenu sous Gordien III un banal "gymnasion*, renforce évidemment l'hypothèse qui y voit un Sérapieion-Asclépieion dans sa première phase et un établissement de cure balnéaire sous le patronage d'Asclépios dans sa seconde phase, à l'èpoque d'Hadrien. 


\section{4. - Le Thestre}

par J.-Ch. MORfTTI

Une seconde campagne de sondage du bâtiment de scène s'est déroulée du 25 mai au 17 juin avec quatre équipes d'ouvriers et la collaboration de $\mathrm{Ph}$. Braillard, stagiaire. S. Diez a effectué plans et relevés (fig. 21); les monnaies, nettoyées par 0 . Didelot et S. Sanders, ont été identifiées par O. Picard.

\section{LE PROSKĖNION.}

Le retour vers l'Est que fait la fondation du stylobate du proskènion est dégagé du côté .Vord, mais son symétrique au Sud est presque totalement masqué par la maçonnerie romaine. Le sondage $87 / 1$, implanté dans un espace réservé de la frons scaenae, a permis d'en examiner partiellement les deux assises de poros et d'en assurer l'emplacement (fig. 22).

Une section du conduit qui traverse du . Nord au Sud la salle A (cf. $B C H 111$ [1987], p. 606, fig. 33) et qui avait été repéré dans la parodos . Nord et la salle D (cf. $B C H 106$ [1982], p. 647 et fig. 16) a été mise au jour dans ce sondage. Il fut construit sur les fondations du proskènion, après le démontage de son stylobate, et postérieurement obstrué et en partie couvert par la première puis la seconde frons scaenae romaine. L'installation de ce conduit n'a donc pu s'effectuer que dans une période d'abandon du théâtre. Le remblai dans lequel il est placé a fourni un matériel assez pauvre (poterie commune et un fragment d'ESA), mais qui confirme la datation que la fouille de la parodos. Nord lui avait attribuée : la seconde moitié du jer siècle ap. J.-C. Dans le conduit on a trouvé une céramique de la première moitié du II $^{e}$ siècle ap. J.-C. (une lampe à anse triangulaire, deux fragments de "red-on-white»), qui fixe un terminus post quem pour l'édification du premier bâtiment de scène impérial (état II).

\section{Le passage souterrain.}

Le passage qui, à l'époque hellénistique, permettait de passer en sous-sol du bàtiment de scène à l'orchestra avait déjà èté repéré en 1955 (cf. $B C H 80$ [1956], p. 386) et en grande partie fouillé en 1956 (cf. $B C H 81$ [1957], p. 644-646). Depuis cette époque, les terres d'alluvion l'avaient presque totalement obstrué. Il a été à nouveau entièrement dégagé (fig. 23), ce qui a conduit à la découverte de l'escalier Nord-Sud qui, de la skènè, menait au couloir souterrain. Les quatre marches inférieures en sont visibles, les quatre autres, qu'il faut restituer pour atteindre le niveau du sol de la skènè, sont noyées sous la maçonnerie de la frons scaenae romaine.

\section{Le postscaenium.}

Dans le sondage 87/II on a mis au jour un nouveau bloc du mur $\mathrm{Pl}^{\prime}$, qui, dans l'état I de l'édifice, soutenait, du côté Est, les terres de la rampe Sud d'accès au proskènion (cf. BCH 111 [1987], p. 605 et fig. 33). Cette assise fut en partie recouverte lors de l'aménagement du posiscaenium de l'état II par les soubassements de son mur méridional, qui fut lui-même ultérieurement arasé, quand la pièce fut étendue d'environ $12 \mathrm{~m}$ vers le Sud (état III). La fouille a fait apparaitre une fondation jetée doublant sur leur face Ouest les assises de poros qui supportaient le mur de fond du portique Est. Ce doublage, attribuable au premier remaniement impérial (état II), s'enfonce à travers deux couches d'occupation MH III pour atteindre le sol vierge.

\section{LA GALERIE Est.}

La grande salle qui, dans le premier état du théâtre, était occupée par un portique adossé à la skènè (cf. $B C H 80$ [1956], p. 386-387; 81 [1957], p. 643), a été sondée à ses extrémités Sud (87/III, fig. 24) et Nord (87/IV, fig. 25). La fondation qui supportait le mur de fond du portique est composée de sept assises de poros, dont la première repose sur le cailloutis rougeâtre qui constitue le sol vierge. Elle ne possède de lien organique ni avec les fondations des murs latéraux du portique, qui comportent trois assises, ni avec celles des murs latéraux de la skènè. Sa hauteur $(2,10 \mathrm{~m})$ et sa position (entre la skènè et le portique) conduisent à penser qu'elle fut édifiée avant les autres soubassements et servit au début des travaux de mur de soutènement pour le remblai sur lequel fut installé le bâtiment de scène. Les sondages $87 / \mathrm{III}$ et $87 / \mathrm{IV}$ ont en effet atteint le sol vierge à $2,30 \mathrm{~m}$ sous le niveau de l'orchestra. Depuis la première occupation du lieu à l'époque mésohelladique (MH III), le niveau du sol s'était élevé d'environ $0,70 \mathrm{~m}$ et il fallut donc remblayer le terrain sur une hauteur de $1,60 \mathrm{~m}$ pour obtenir un terrain plat, apte à recevoir le nouvel édifice. Ce remblai, dont la partie supérieure fut bouleversée par des recreusements du ve siècle ap. J.-C., a fourni un abondant matériel céramique dont les éléments les plus récents 
Fig. 21. - Théâtre. Bătiment de scène. Plan de situation des sondages 1987 (S. Diez; J.-Ch. Moretti).

Fig. 22. - Plan du sondage 87/1. En grisé : fondations de poros du proskènion (S. Diez; J.-Ch. Moretti).
Fig. 23. - Le passage souterrain. Le couloir vu de l'Ouest (photo Ph. Collet). 


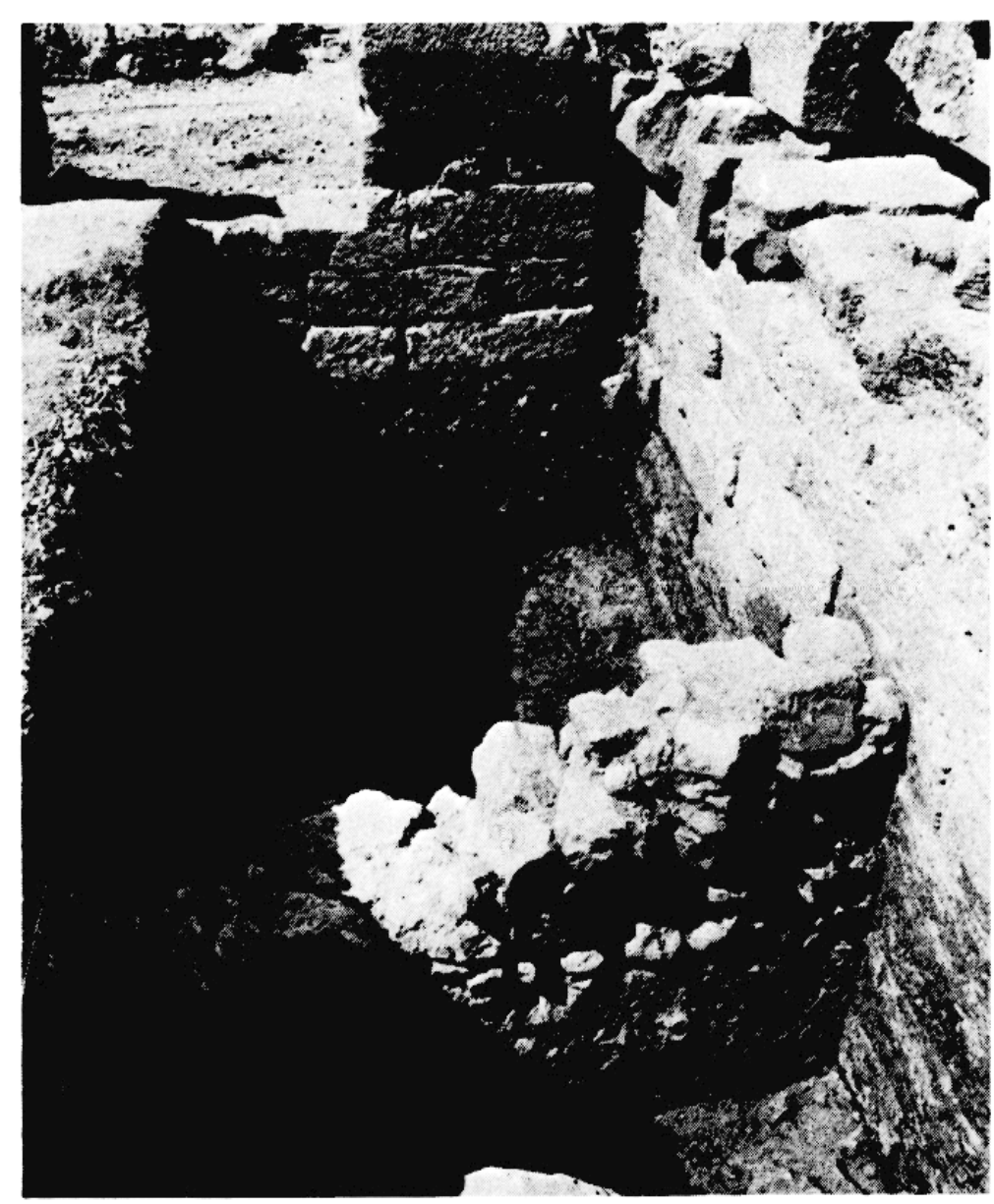

Fig. 24. - Galerie Est. Sondage 87/IIIa vu du Nord.
Illustration non autorisée à la diffusion

Fig. 26. - Figurine en terre cuite : Attis (inv. 87/5/10) (photo Ph. Collet).

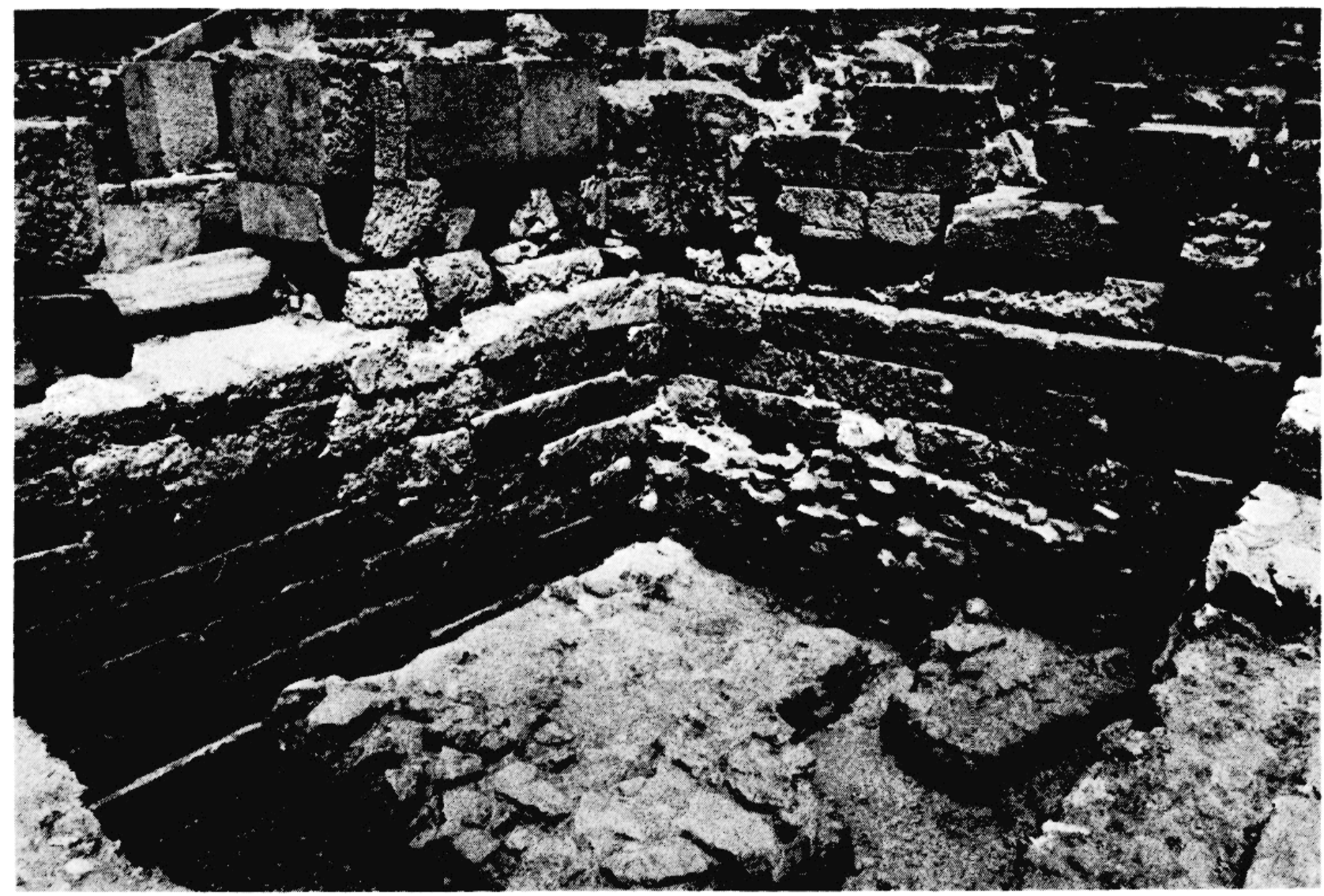

Fig. 25. - Galerie Est. Sondage 87/IV vu du Sud-Est. 
Fig. 27. - Attache d'anse d'hydrie en bronze : bélier, face (inv. 87/23/1) (photo Ph. Collet).
Fig. 28. - Pied de coupe attique inscrit (inv. 87/143/1) (photo Ph. Collet).

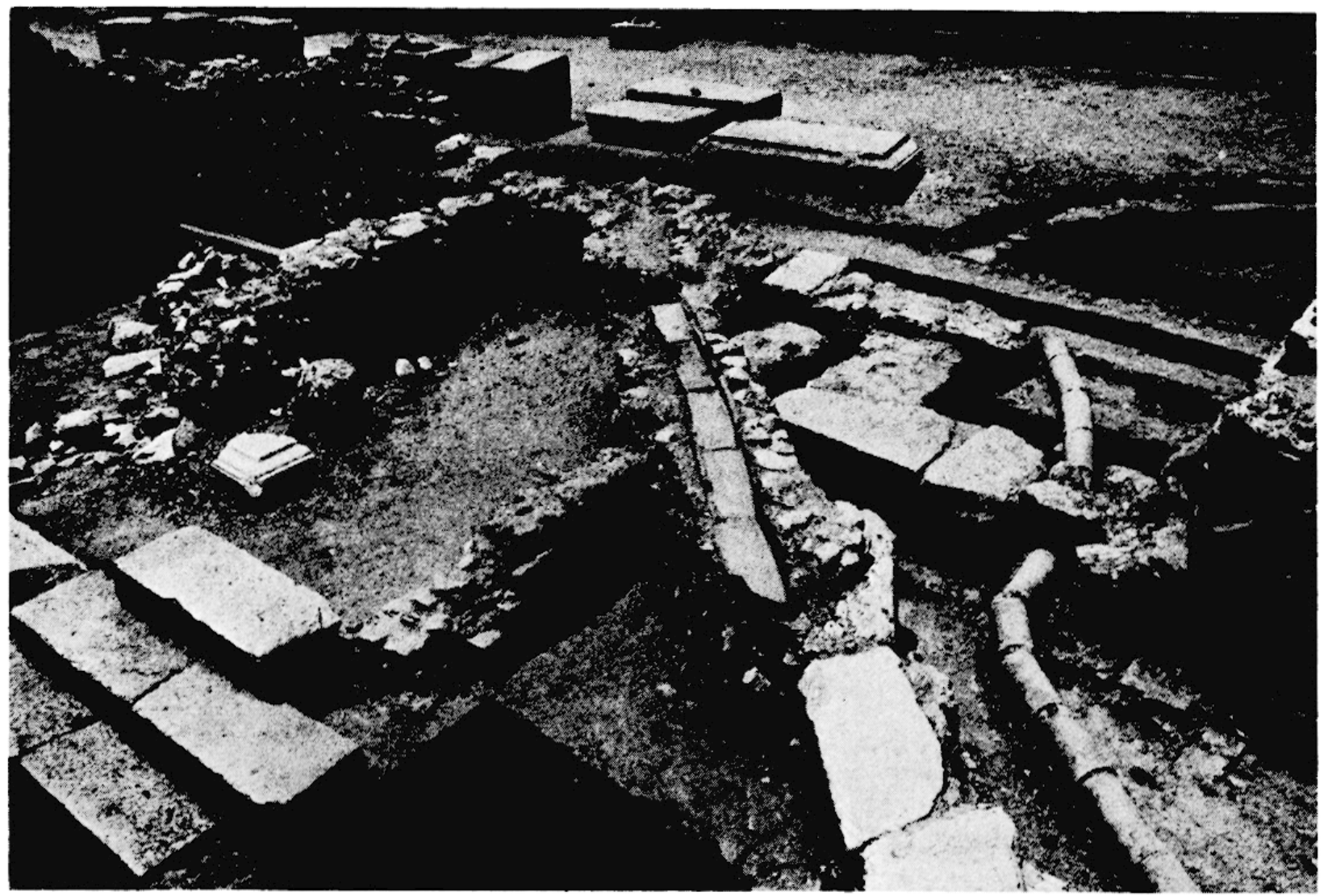

Fig. 29. - Passage entre le théâtre et les thermes A, vu du Nord-Est. 
datent du début du nie siècle av. J.-C. Cette datation est confirmée par la présence dans ce remblai d'une litra frappée par Agathoclès, roi de Syracuse (304-289 av. J.-C.).

La mise en chantier par les Argiens d'un nouveau théâtre beaucoup plus grand que leur théâtron à gradins droits et doté d'un bâtiment de scène adapté aux exigences des spectacles du temps découle sans doute de leur mainmise sur les concours Néméens à la fin du Ive siècle av. J.-C. ${ }^{35}$. Son achèvement permit le transfert des concours à Argos au cours du IIre siècle av. J.-C.. ${ }^{36}$.

Dans le sondage $87 / \mathrm{III}$, dont seule la partie Ouest (87/IIIa) a pu être cette année totalement fouillée, on a commencè à mettre au jour une fondation de petits moellons et de mortier qui se développe à $2,75 \mathrm{~m}$ au Nord du mur méridional de la galerie et parallèlement à lui. Aucun matériel n'est associè à cette fondation, qui fut jetée dans une étroite tranchée $(I=0,55 \mathrm{~m} \pm)$. Il faut vraisemblablement l'associer avec le mur qui, dans l'état. II, recouvrit. sur $3,48 \mathrm{~m}$ l'extrémité Sud de la fondation Est de la galerie. Si la poursuite de la fouille confirme cette hypothèse, il conviendra de restituer dans l'état II, à l'extrémité Sud du portique, une petite pièce $(2,75 \mathrm{~m}$ Nord-Sud $\times 5,50 \mathrm{~m}$ Est-Ouest).

Notons enfin la découverte, dans la couche supérieure de $87 / \mathrm{III}$, d'une statuette en terre cuite (Ht. conservée : $21 \mathrm{~cm}$ ) représentant, semble-t-il, Attis appuyé à un pedum (fig. 26) et celle, dans la tranchée de fondation du mur septentrional du portique, d'un avant-train de bélier couché en bronze ( $\mathrm{L}$ cons. $=3,2 \mathrm{~cm})$, extrémité de l'attache inférieure d'une anse d'hydrie de la seconde moitié du vie siècle av. J.-C. ${ }^{37}$ (fig. 27).

\section{La salle G.}

Dans la salle $\mathrm{G}$, le sondage $87 / \mathrm{V}$ a rencontré à $0,86 \mathrm{~m}$ sous le niveau de l'orchestra un sol de mortier très résistant associé aux fondations de poros du mur Nord du portique Est. La face Nord des deux assises supérieures de la fondation, qui s'élèvent au-dessus de ce sol, est stuquée. La différence de niveau entre ce sol et celui du båtiment de scène hellénistique - équivalent à celui de l'orchestra - doit être mise en rapport avec la fosse emplie de statuettes découverte dans la salle $F^{38}$. A n'en pas douter, quand le théâtre fut édifié, cette zone était déjà occupée et très vraisemblablement consacrée à quelque divinité. Cela expliquerait que le portique accolé à la skènè ait été déporté vers le Sud par rapport à l'axe Est-Ouest de l'ensemble du théâtre.

Parmi le matériel découvert sous le niveau du sol de l'actuelle salle $\mathrm{G}$, il convient de signaler un pied de coupe attique (fig. 28) du type Agora XII, 420 (daté de 500-480 av. J.-C.) sur lequel est gravé :

\section{ФAGNIIIO}

Les hastes du epsilon sont orientées vers la gauche; l'écriture semble peu postérieure à la fabrication du vase ${ }^{39}$.

\section{LE PASSAgE ENTRE LE THÉÂtRe fT LES THERMES A.}

Le projet de réaménagement de la rue du théâtre (cf. $B C H 111$ [1987], p. 607) a rendu urgente la fouille du passage qui sépare le théâtre des thermes $A$ (sondage $87 / \mathrm{VI}$ ). Elle a permis de préciser les différentes formes que prit l'accès de la rue à la parodos Sud pour répondre aux modifications successives des deux monuments (fig. 29). Lors de la construction du théâtre (au premier tiers du u $u^{e}$ siècle av. J.-C.), un large escalier, composé de six marches dont trois sont en partie conservées in situ (cf. $B C H 81$ [1957], p. 368 et en B sur la figure 2), fut installé pour mettre en relation la placette située à l'extrémité Ouest de la rue et la parodos Sud, située à environ $1,20 \mathrm{~m}$

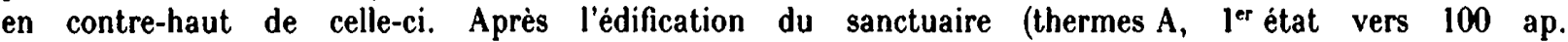
J.-C.), l'escalier fut réduit à une largeur de $3,50 \mathrm{~m}$, limité par deux murs d'échiffre en opus caementicium. Au milieu du nıe siècle ap. J.-C., ce système de circulation, rendu inutilisable par l'érection de la salle A 2 des thermes A, fut remplacé par un nouvel escalier situé au Nord-Ouest du précédent, accolé au mur Sud du postscaenium.

(35) Cf. P. Amandry in BCH, Suppl VI (1980), p. 247 ; M. Piénart, J.-P. Thalmann, ibid., p. 266-269; S. G. Miller in Hesperia, Suppl XX (1982), p. 106-107; Hesperia 57 (1988), p. 11 et 18.

(36) A la fin du in'e siècle av. J.-C., les concours en l'honneur de l'Héra argienne furent aussi transférés de l'Héraion à la cité : cf. P. Amandry in BCH, Suppl VI (1980), p. 226-231 et 244-247.

(37) Cf. L. Politis, ArchEph 1936, p. 147-174; Cl. Rolley, Les vases de bronze de l'archaïsme récent en Grande Grèce (1982), p. 31-47.

(38) Cf. BCH 106 (1982), p. 647; 107 (1983), p. 839-840; M. Guggisberg, BCH 112 (1988), p. 167-234. Lors d'un nettoyage effectué cette année pour complèter le plan de la fosse, de nouveaux moules et statuettes ont été découverts (cf. ibidem, p. 535 sq.).

(39) Le nom de Phainippos est attesté à Argos à l'époque hellénistique, cf. O. WALter, OJh 14 (1911),

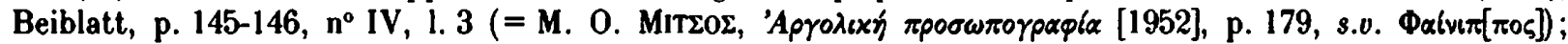
M. Pierart, BCH 104 (1980), p. 696 (=SEG XXX [1983], n³71). 


\section{DELPHES}

Fig. 1. - Christos Kaltsis, contremaitre de l'EFA à Delphes (cl. J. Bousquet).

Christos Kaltsis est mort le 5 septembre 1988 (fig. 1). Il avait èté nommé contremaitre des fouilles de l'École française à Delphes en 1936, à l'àge de 25 ans; il a dirigé les travaux de l'École pendant quarante ans, jusqu'à sa retraite en 1976, à Delphes mème et sur d'autres sites de Phocide et de Béotie (Kirrha de 1936 à 1938, Médéon en 1962, le Ptoïon de 1963 à 1965, l'Antre corycien en 1970 et 1971), et occasionneltement à Philippes et à Argos. Le déblaiement en 1936 de la partie du sanctuaire d'Apollon recouverte par une coulée de terre et de rocaille venue des Phédriades, le dépavage en 1938 et 1939 de la voie sacrée, le rassemblement des éléments nécessaires à la restauration des colonnes de la tholos de Marmaria et du temple d'Apollon, furent les principaux travaux conduits à Delphes au cours des années qui précédèrent la guerre. Après avoir participé à la campagne d'Albanie, Christos Kaltsis passa à Delphes les huit années d'occupation étrangère et de guerre civile, vivant en ermite auprès des ruines, qu'il contribua largement à préserver de dommages graves. La reprise de l'activité archéologique à partir de 1950 lui permit de s'épanouir pleinement dans l'exercice de ses fonctions. Sa vigueur physique, son coup d'œil, son sens de l'économie des efforts dans le maniement des plus gros blocs, lui assuraient une autorité incontestee sur les ouvriers. Il avait appris au fil des ans à connaître les pierres de Delphes aussi bien que les archéologues, et sa curiosité constamment en éveil lui fit découvrir maints fragments d'inscriptions et d'éléments architecturaux dans les murs des terrasses entre les sanctuaires d'Apollon et d'Athéna et le lit du Pleistos. C'est lui qui a attiré l'attention sur un site préhistorique dans la vallée du Pleistos, sur des carrières antiques autour de Delphes, sur le site des Marmara et d'autres sites antiques sur le plateau du Parnasse. Malade les dernières années, il revenait quand il pouvait jeter un coup d'œeil sur les ruines, auxquelles il a consacré sa vie sans partage. 


\section{Le xyste}

par Évangélos Pentazos, Vincent Déroche et François Queyrei.

Les travaux de la fouille franco-hellénique se sont déroulés du 29 juin au 24 juillet, sous la surveillance d'E. Pentazos, de V. Déroche, au début et à la fin de la campagne, et de $F$. Queyrel, en présence aussi d'A. Coccaliou. Y. Lafond, J. Varalis et V. Visa ont été assistants de fouille; relevés et dessins ont été confiés à D. Laroche, qui a travaillé avec deux collaborateurs, J.-B. Bellon et J.-F. Carlotti, ainsi qu'avec deux topographes, Ph. Nivet et A. Osmo. Le contremaître D. Koritos était présent pour l'ensemble des travaux, qui ont employé une quinzaine d'ouvriers. Les interventions de restauration ont été confiées à D. Deyber et C. Macquet.

\section{A. Secteur centre-Nord du portique du xyste (BC 25 28).}

De nouveaux élèments des dernières phases du secteur ont été reconnus.

1. Un sol de chaux tassée avec du tuileau et quelques gravillons a été repéré en BC 27 , à un niveau inférieur de quelques centimètres au talon du mur de fond. Il n'a pu être fouillè cette année.

2. La couche suivante supporte des blocs rangés à une trentaine de centimètres au-dessus du niveau de ce sol, le long du mur de fond, notamment deux fragments importants de colonnes d'époque impériale du xyste, couchès contre le mur, ainsi que quelques blocs en calcaire du mur de fond (fig. 2). Une partie de la colonnade et du mur de fond était donc déjà endommagée dans un autre secteur. D'après la céramique retrouvée, ce rangement date au plus tôt du $\mathrm{v}^{\prime \prime} \mathrm{s}$. ap. J.-C.

3. La trouvaille en BC 26 d'un ensemble de blocs tombés du mur de fond à un niveau proche de celui du sol mentionné au $\S 1$ indique que la ruine du mur dans ce secteur a suivi de peu la période du rangement des blocs. Signalons que certains de ces blocs tombés portent des traces d'enduit peint.

4. Une èpaisse couche d'èpandage contient un matériel en majeure partie tardif, ainsi que quelques ratés de cuisson et colifichets.

5. Un âtre grossier (pierres et briques) a été installé en $\mathrm{BC} 27$ dans la couche d'épandage, à une date indéterminée.

\section{B. Secteur médian du xyste (B 21 \̀ 23; D 18-19).}

a) Mur de fond (B 21 a 23) :

La fouille de la berme laissée le long du mur de fond a permis de retracer une évolution différente dans ce secteur de celle que nous avons constatée plus au Nord.

1. On note l'absence de rangement de blocs et celle d'un sol au niveau du talon du mur. Le remblai inférieur est une terre meuble sans cailloutis, qui contient un matériel dont les éléments les plus récents datent au plus tôt du ves. ap. J.-C. Le mur a connu dans ce secteur une destruction naturelle légèrement antérieure à celle que l'on constate plus au Nord.

2. Un épandage analogue à celui du secteur Centre-.Nord, comprenant quelques blocs du mur de fond, a recouvert la couche précédente.

b) Stylobale (D 18-19) :

La berme qui recouvrait le stylobate et le caniveau a été fouillée.

1. Une série de piédestaux du xyste de l'époque impériale a été trouvée en place (fig. 3).

2. Une couche de chaux, mêlée de scories, de ratés de cuisson et de céramique tardive, recouvrait le caniveau en s'appuyant sur le stylobate. Ce sol a été simplement dégagé.

3. Une épaisse couche d'épandage, contenant du matériel de la fin de l'Empire romain, a été repérée audessus. 


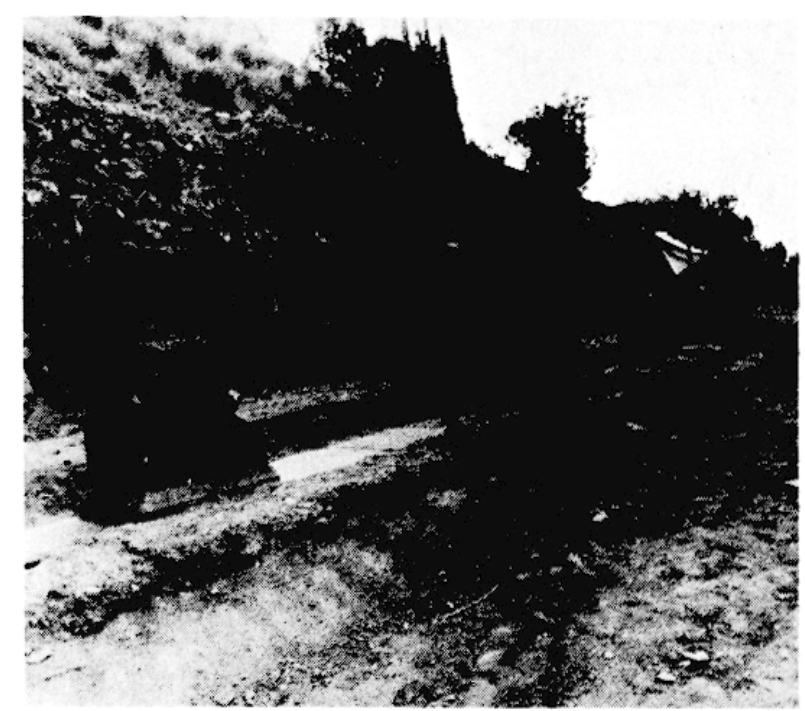

Fig. 3. - Stylobate et piédestaux du xyste, en CD 14-15 (cl. Ph. Collet).

Fig. 2. - Rangement d'une colonne contre le mur de fond, en B 26 (cl. F. Queyrel).

Fig. 4. - Retour du mur d'analemma de la palestre, second four et citernes, dans le secteur Sud de la paradromis (cl. Ph. Collet). 


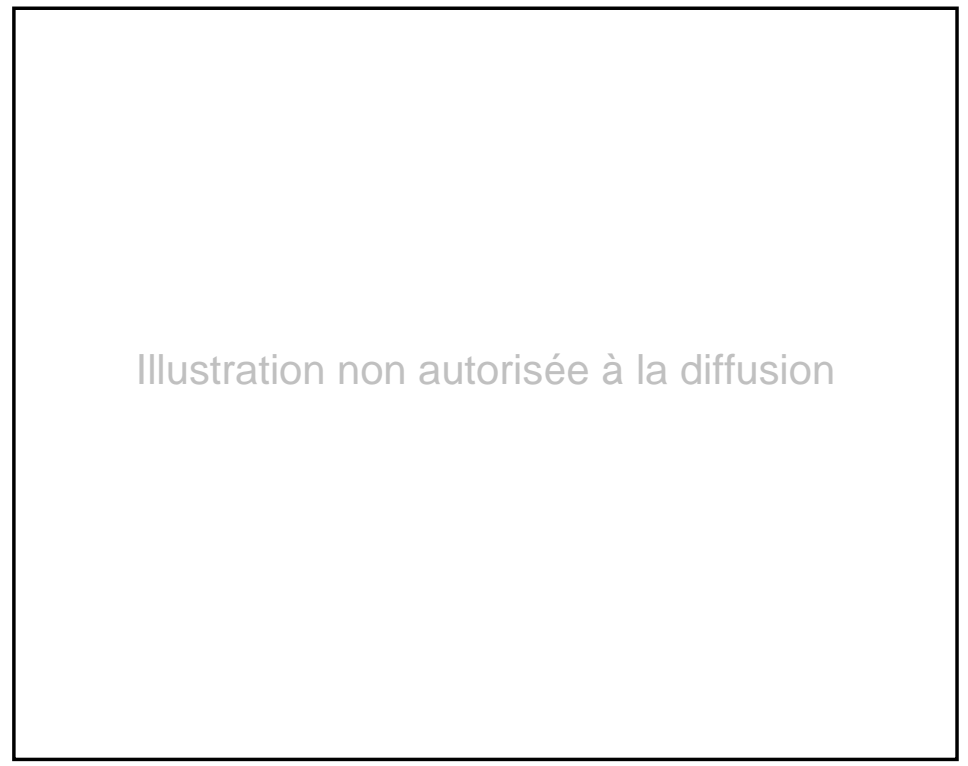

Fig. 5. - Détail du bloc inscrit remployé dans un mur du four (cl. Ph. Collet).

\section{Illustration non autorisée à la diffusion}

Fig. 6. - Squelette dans la citerne effondree (cl. Ph. Collet).

Fig. 7. - Pièce sommitale du larmier d'un fronton du temple en calcaire, vue en contre-plongée (cl. $\mathrm{Ph}$. Collet).

\section{Paradromis Sud.}

Les fouilles de l'an dernier ont été ètendues dans ce secteur vers le . Vord ou vers l'Ouest.

1. On a reconnu sur environ $3 \mathrm{~m}$, dans les carrés $\mathrm{G}$-II 8 , le retour en appareil polygonal grossier du mur d'analemma de la palestre, déjà dessiné dans J. J AnNoraY, FI) II, Le gymnase, pl. II (fig. 4). Une épaisse couche de cailloutis, repérée en $\mathbf{G} 8-9$, doit dater de l'aménagement de cette terrasse.

2. Un deuxième four, analogue à celui trouvé lors des campagnes précédentes, est apparu en G 9-10. I,a structure, orientée . Nord-Sud, comporte sur son long cỏté Est un mur en briques de mème appareil que le four déjà connu; son extrémité Sud comprend un bloc de poros en remploi qui porte les lettres $\wedge$ AV en écriture archaïque (fig. 5). L'entrée Sud de la chambre a été bouchée par un mur de pierres sèches. On constate un arasement de la structure, dont la fouille reste à faire. 
3. Une couche d'épandage contenant essentiellement du matériel des ve et vi s. ap. J.-C., notamment des ratés de cuisson, des colifichets et une grande quantité de tuiles et briques (surtout en G 11-12) a recouvert l'ensemble du secteur.

4. Deux citernes ont èté reconnues en G 10-11. La première avait déjà été repérée (J. JANNORAY, o.c. p. 16 pl. XIII.3); il ne s'agit pas d'une "tombe byzantine" à l'origine, mais d'une citerne, comme le prouve la présence de restes de canalisations et d'enduit hydraulique à l'intérieur. La seconde, partiellement fouillée anciennement (J. Jannoray, loc. cit.), jouxte la précédente à l'Ouest; ses murs sont détruits au-dessus du départ de la voute. L'utilisation de cette structure comme citerne est confirmée par le remploi à l'Ouest de deux éléments de caniveau en calcaire, semblables à ceux trouvés en bordure Ouest d'une partie de la paradromis. Les deux citernes, qui ont coupé le four, recouvrent son radier. Un mur Sud-.Nord est liè à ces citernes en F 9-10.

5. La citerne effondrée a èté réutilisée comme tombe : on y trouve un squelette sans mobilier funéraire (fig. 6). La fouille est arrètée à ce niveau. Cette réutilisation est sans doute lièe à l'implantation d'un cimetière en bordure de la paradromis. Une tombe apparaît en effet en G 11, creusée dans la couche d'épandage. Elle est couverte de tuiles entre deux rangées de pierres posées de chant et contient un matériel très pauvre, quelques restes de bijoux en bronze.

6. Sur le côté Est du four mentionné $\S 2$, un mur de soutènement est, dans l'ètat d'avancement de notre fouille, attribuable au mème ensemble que les fondations arasées en moellons et mortier (BCH 110 [1986], p. 774 : ètat II).

7. Un amas de pierres sèches apparait à l'extrémité Est du retour du mur d'analemma de la palestre. II date sans doute d'un sondage mené par Jannoray.

Signalons pour terminer quelques trouvailles : la pièce sommitale du larmier d'un fronton du temple en calcaire, dans le carré $F \mathbf{9}$ (fig. 7); un fragment de doigt en marbre venant d'une statue deux fois plus grande que nature (l'Athéna du temple en tuf de Marmaria?).

\title{
PHILIPPES
}

\author{
par Michel SÈve
}

Les travaux poursuivis au forum de Philippes ont consisté en une campagne d'ètude du matériel architectural menée du 3 aoùt au 9 septembre, avec la collaboration de MM. Patrick WeBER, architecte, Thierry Sемвасн, stagiaire d'architecture, et Vélissarios Anagnostopoulos, dessinateur; nous avons en outre reçu le concours de deux stagiaires du certificat d'études approfondies * Architecture et archéologie * animé par les écoles d'architecture de Nancy, Paris et Strasbourg, M ${ }^{\text {lles }}$ Marie Revel et Manuela Wurch. Enfin M. Dimitrios Korıtos, venu de Delphes avec un engin élévateur, a consacré trois semaines à la manutention et au rangement des blocs.

Le but principal de la campagne ètait d'avancer, et si possible de terminer, les dessins de blocs et les constatations matérielles. Il a été en grande partie atteint. L'effort a surtout porté sur le dossier graphique des monuments ornant le cóté de la place dominé par le decumanus maximus ( $\left.n^{\circ} 3\right)^{1}$, essentiellement la tribune 2 et les "chapelles 4 et 26 . Des restitutions partielles ont pu en étre élaborées : elles confirment qu'il s'agit de monuments très petits, d'une exécution sensiblement moins soignée que celle des grands temples Est et Ouest; ils sont en outre beaucoup moins bien conservés. Le dossier des fontaines 5 et $\mathbf{2 5}$ a èté complété, et leur étude

(1) Les numéros renvoient au plan avec légendes publiẻ $B C H 106$ (1982), p. 651-653. 


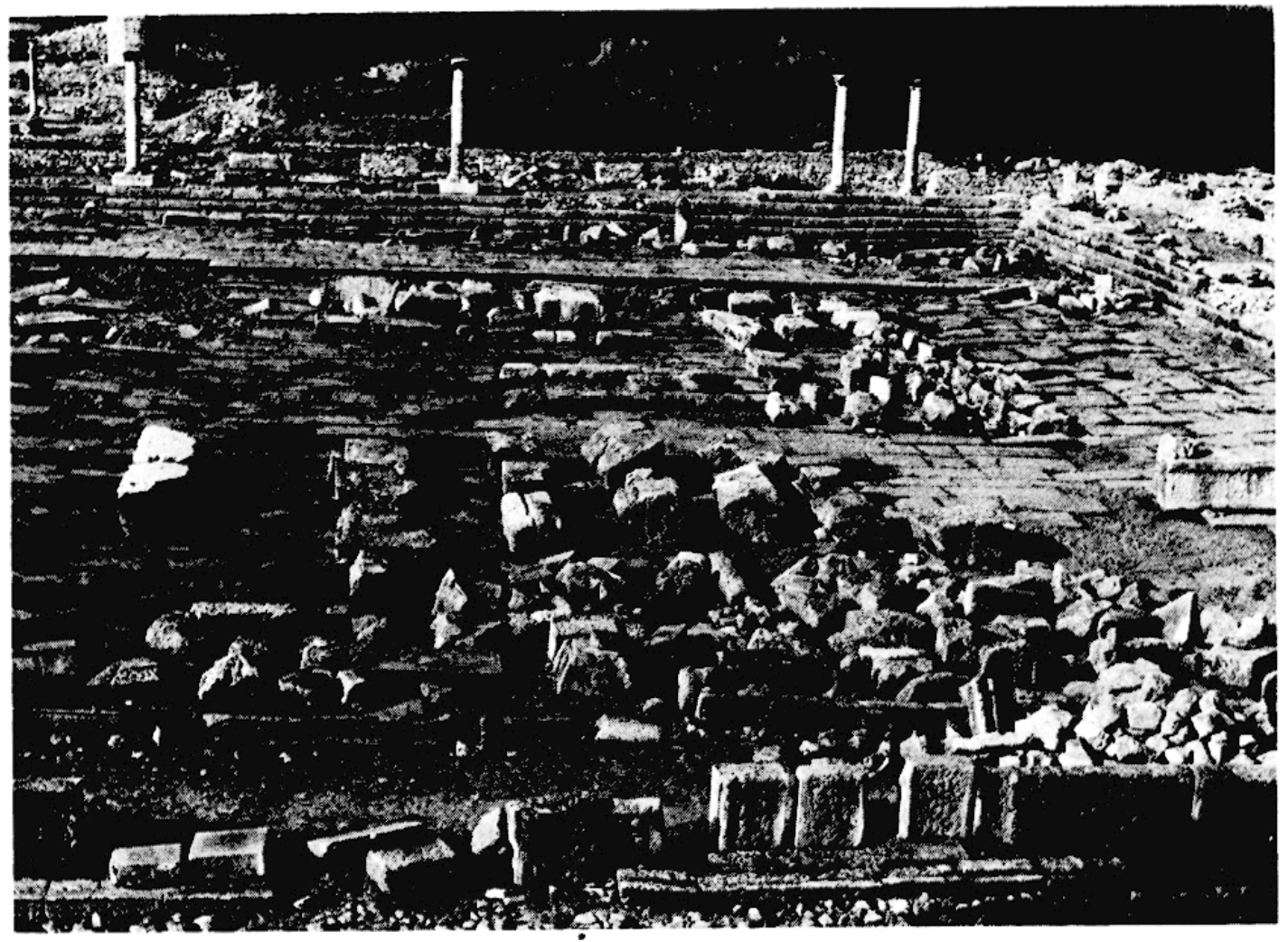

Fig. 1. - Blocs d'architrave et de corniche appartenant au temple Ouest.

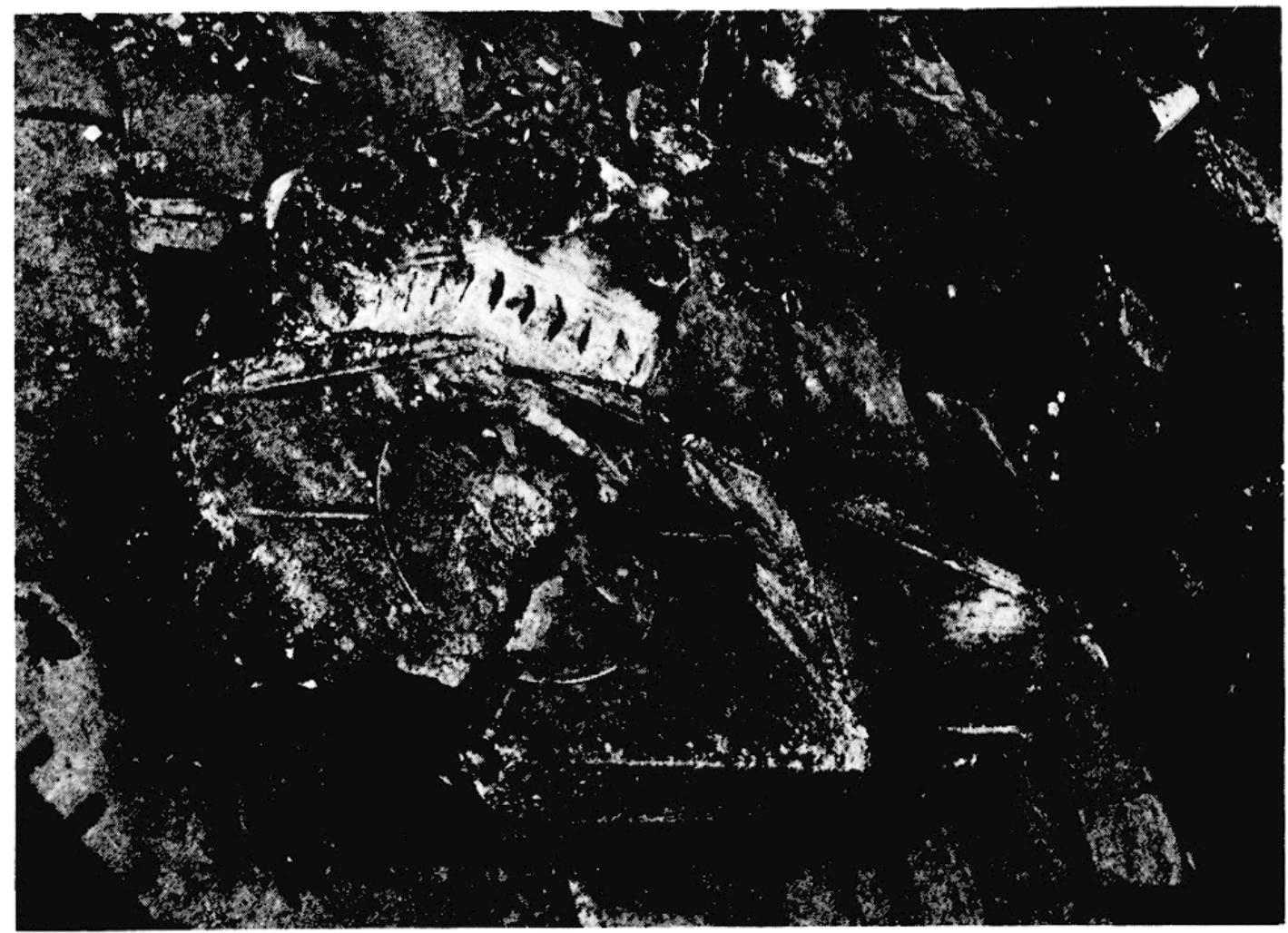

Fig. 2. - Tympan et sommet des rampants du temple Ouest. 
avancée : il semble bien qu'il s'agisse au moment de leur construction, de bassins à fonction plus décorative qu'utile, ornés en leur centre d'un pilier de grand appareil portant à son sommet une tête de lion verseuse d'eau. En revanche, les plaques inscrites trouvées dans la fontaine Ouest (5) et qu'on attribuait à son èlévation, ne peuvent y trouver place : elles proviennent de deux monuments honorifiques différents, plus anciens qu'elle, dont l'emplacement est à chercher au centre de la place; de même, appartient à un monument de ce genre une grande architrave inscrite que l'on avait pensé attribuer à l'un des monuments 4 ou 26 et qui n'est compatible avec aucun d'entre eux.

De la même façon, on a constitué le dossier graphique des éléments appartenant à des arcs, dont les arcs 7 , 9 et 24 , sans avoir encore abordé leur restitution. Il en va de même pour la "basilique 12 et le "monument incendié " 13, encore en cours d'étude. L'examen détaillé des murs paléochrétiens a permis d'y repérer en remploi de nombreux fragments de chapiteau, d'épistyle et de corniche dont nous supposons qu'ils appartiennent à l'élévation, perdue, des portiques 11, 14 et 19 dans leur état antonin tardif : ce sera l'objet de la prochaine campagne que de les étudier précisément. Des compléments ont en outre été apportés aux dossiers de la bibliothèque 18 et des boutiques 15, et un travail patient amorcé sur les blocs courants des murs d'ante des temples Est et Ouest (10 et $\mathbf{2 1}$ ) permettra d'en restituer le montage, et devrait donner des indications sur leur hauteur, seul élément d'incertitude dans la restitution de leur façade.

On a d'autre part achevé la description détaillée des vestiges actuels en examinant les bâtiments du cỏté Sud de la place (où l'on a pu reconnaitre l'organisation de l'état le plus ancien, similaire à celle qui avait déjà été reconnue sur les petits cỏtés Est et Ouest), et des monuments bordant au . Nord le decumanus maximus.

Enfin, des travaux de rangement des blocs ont été exécutés à la bibliothèque, où les fragments qui encombraient le centre de la pièce ont été rassemblés par séries le long des murs; les blocs appartenant à l'épistyle inscrit ont été présentés dans l'ordre, face au monument. L'essentiel du travail a été consacré au dégagement et à un début de présentation des abords du temple Ouest. Cette zone, que l'amoncel!ement des blocs rendait totalement inaccessible avant les travaux exécutés en 1979, 1980 et 1982 , est maintenant commodément visitable. Le poids de certains blocs n'a certes pas permis de dégager complètement le portique 11. Mais l'esplanade devant le temple a été complètement libérée, les élèments de l'élévation du temple rassemblés par séries (fig. 1) et l'on a tenté une présentation à plat du tympan et du sommet des rampants (fig. 2). On a pu mesurer à cette occasion ce que serait l'extrême difficulté d'éventuels travaux d'anastylose, à cause du poids des blocs et de leur mauvais état général de conservation.

\title{
DIKILI TASH
}

\author{
par Katérina Péristéri et René Treuil
}

Sur le site préhistorique bien connu de Dikili Tash, le programme de recherches franco-hellénique, qui avait commencé à entrer en application en 1986 (cf. Ergon 1986, p. 53-57, et BCH 111 [1987], p. 616-619), s'est poursuivi cette année par une seconde campagne de fouilles, dirigée par H. Koukouli-Chryssanthaki et R. Treuil. Financés par la Socièté archéologique grecque, l'École française d'Athènes et le Ministère français des Affaires Etrangères, ces travaux se sont déroulés du 7 septembre au 2 octobre, avec la participation d'une vingtaine de personnes : étudiants, chercheurs et techniciens grecs et français ${ }^{1}$.

(1) L'équipe comprenait, du côté grec : H. Koukouli-Chryssanthaki, K. Péristéri, E. Gérondakou, Vaïa Ikonomidou, A. Koukouvou, D. Malamidou, Str. Papadopoulos et I. Tournavitou, archéologues; du côté français : R. Treuil, P. Darcque, A. Farnoux, M.-H. Georgiadou, L. Karali-Yannacopoulos, G. Kourtessi-Philippaki, Chr. Marangou, O. Polychronopoulou, J. Renard, archéologues; L. Faugères, géographe; R. Étienne et J.-Ph. Guyon, topographes; V. Anagnostopoulos, dessinateur et Ph. Collet, photographe. 


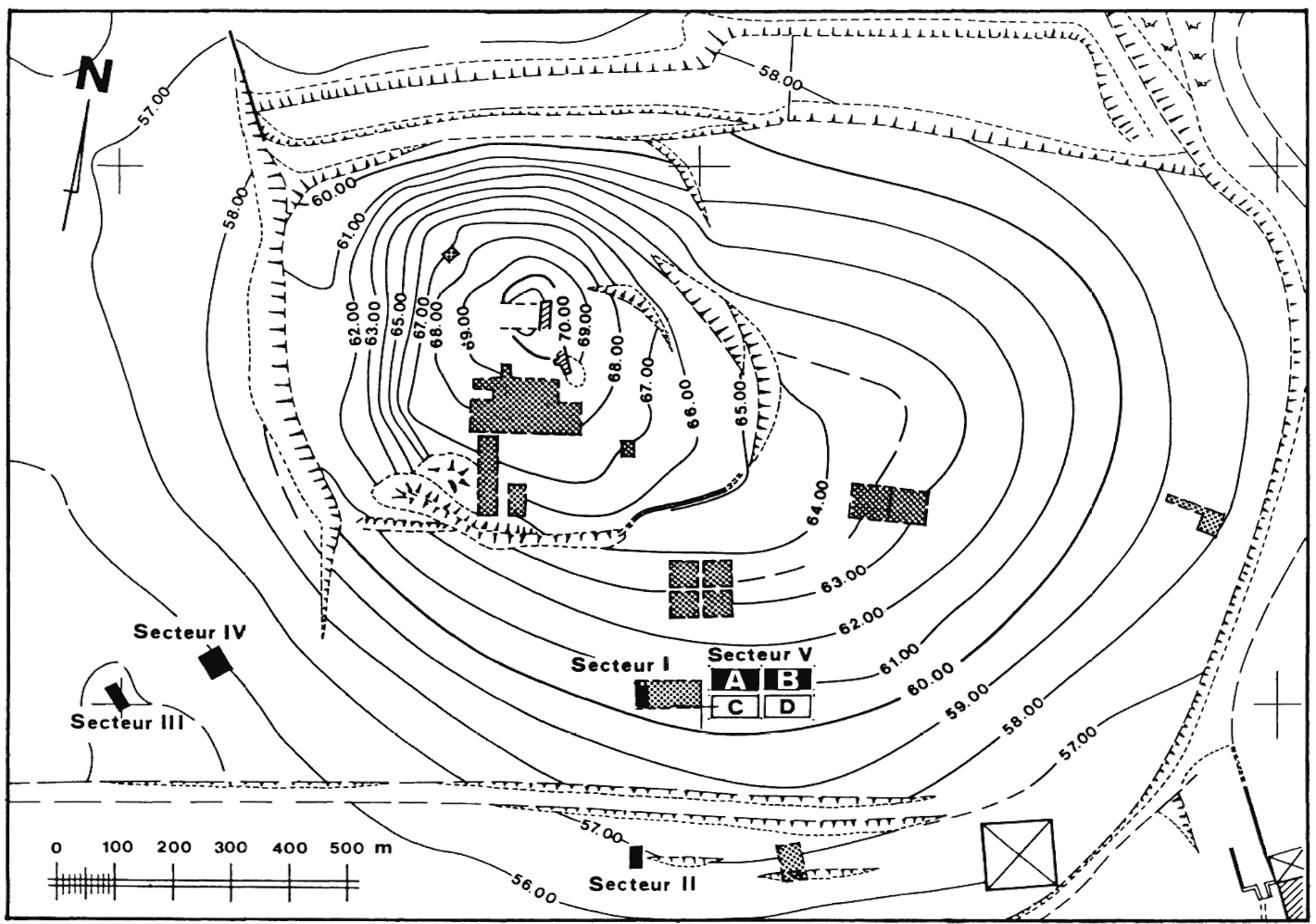

Fig. 1. - Dikili Tash. Plan topographique simplifié; en grisé les fouilles de 1961 à 1975; en noir les secteurs ouverts depuis 1986 . 
La remise en état de la maison de fouille, effectuée pour l'essentiel en 1986, a été achevée. La clôture métallique a été agrandie de façon à englober la totalité des terrains expropriés en 1961, c'est-à-dire, à peu de chose près, l'ensemble du site (fig. 1).

Les travaux effectués cette année sont de trois ordres : topographique, géomorphologique, archéologique.

Un plan topographique au $1 / 500^{\circ}$, couvrant une superficie de 25 ha, avait été réalisé en 1986 . Il a été étendu cette année, au $1 / 1000^{\circ}$, sur plus de 60 autres ha, en direction du . Nord, de l'Ouest et du Sud. En outre, on a réalisé un nouveau plan, au $1 / 200^{e}$ cette fois, de la partie sommitale du tell : il s'agissait de préciser le tracé du mur de pierres sèches qui avait été repéré depuis longtemps sur la pente Sud et sa relation éventuelle avec les bâtiments en pierre dont les ruines sont visibles au sommet. Cette opération, qui a nécessité d'importants travaux de débroussaillage et de nettoyage, a permis de constater que le mur en question présentait bien un tracé grossièrement circulaire et qu'il ètait doublé, sur la pente orientale, par un second mur parallèle. Mais il n'a pas été possible de le retrouver sur la pente occidentale; on a rencontrè là, en revanche, des vestiges de constructions apparemment récentes, ainsi que, au bas de la pente, deux blocs de marbre portant un décor sculpté.

L'ètude géographique entreprise en 1986 par L. Faugères, a été poursuivie cette année. Il s'est attachè plus particulièrement à relier l'étude du site à celle du cadre régional. On a repris en outre l'un des deux sondages - désigné désormais sous le nom de secteur $I V$ (coordonnées 215-220/105-110) - ouverts en 1986 à la base SudOuest du tell; on l'a élargi et approfondi, de manière à accéder, pour la première fois, à des couches situées sous la surface de la nappe phréatique. Des échantillons ont pu ètre ainsi prélevés, à des altitudes définies, en vue d'une étude sédimentologique.

La stratigraphie dans la partie Sud de ce sondage suggère fortement l'alternance de couches archéologiques et de couches limoneuses, comme si les variations du niveau du lac ou du marais avaient entrainé des déplacements correspondants de l'habitat : ce dernier aurait pu s'étendre pendant les phases plus sèches, mais aurait été contraint de reculer pendant les phases plus humides.

Les recherches archéologiques ont été conduites en différents endroits. Tout d'abord, dans le secteur IV dont il vient d'être question, deux points sont à signaler. D'une part, le niveau de pierres qui avait été rencontré l'an dernier à l'altitude de $54 \mathrm{~m}$ s'est révélé plus large qu'on ne l'avait cru; mais on ne l'a pour l'instant pas fouillé et sa datation, comme sa fonction, demeurent donc largement incertaines. D'autre part, parmi les niveaux archéologiques qui alternent, au-dessous des pierres, avec des niveaux limoneux, certains paraissent contenir des lambeaux de couche de destruction encore assez compacts, mais d'autres ne renferment que des traces de torchis brûlé réparties de façon très homogène : ces derniers pourraient avoir été remaniés par l'action de l'eau. Les quelques tessons identifiables paraissent attribuables au Bronze Ancien, ce qui est assez inattendu à une altitude $-53,55 \mathrm{~m}$ - aussi basse.

Dans le secteur II (coordonnées 288-289/070-073), tranchée de $4 \times 2$ m ouverte en 1986 près de la base Sud du tell, la fouille a èté poursuivie en profondeur dans l'espoir d'atteindre les couches archéologiques les plus anciennes. Le lit de pierres rencontré en 1986 à l'altitude de $56,25 \mathrm{~m}$, a été démonté sans que l'on ait pu, dans le faible espace disponible, le dater ni en déterminer la fonction. Au-dessous, la fouille n'a rencontré, jusqu'à une altitude de $55,20 \mathrm{~m}$, que des niveaux perturbés, où quelques tessons historiques et des vestiges peut-être contemporains voisinaient avec de la céramique préhistorique. Une nouvelle zone de pierres, apparemment tombées et dispersées, est apparue dans la moitié Sud du secteur, entre les altitudes 55,20 et $55,60 \mathrm{~m}$. Il se pourrait donc que la fouille n'ait traversé pour le moment, entre 57 et $55,20 \mathrm{~m}$, que des dépôts de pente issus du tell lui-mẻme.

Dans le secleur I (coordonnées 289-291/100-104), où l'on avait repris en 1986, par une tranchée de $4 \times 2 \mathrm{~m}$, l'ancien sondage Ouest de AA 28, la fouille avait également pour objectif, à l'origine, d'atteindre les couches les plus anciennes. Un four du Néolithique Moyen, qui avait été découvert en 1972, a été démonté (fig. 2): on a pu ainsi étudier la façon dont il avait èté construit, puis refait. A l'altitude de 57,24 m est apparu le sommet d'une couche d'incendie d'un état antérieur, comprenant des portions d'un toit apparemment en terre crue, fortement brúlées. Au-dessous encore, une couche de terre très noircie et un amas de torchis brûlé correspondent peut-être à une couche d'incendie antérieure. La fouille s'est arrêtée vers l'altitude $56,80 \mathrm{~m}$.

A $11 \mathrm{~m}$ à l'Est du secteur I a èté délimitée une surface de $9 \times 17 \mathrm{~m}$, appelée secteur $V$ et destinée à faire l'objet d'une fouille extensive : l'objectif est en effet ici de dégager un ensemble d'habitations du Néolithique Moyen et donc d'éclairer par là un aspect jusqu'ici extrêmement mal connu de la préhistoire de la Macédoine. Dans un premier temps, seule une surface continue de $4 \times 17 \mathrm{~m}$ (coordonnées 302-318/103-106) a été ouverte. On y a rencontré, immédiatement au-dessous d'une couche superficielle très ameublie par les cultures, d'importants amas de torchis brûlé, également très perturbés par les labours, mais qui proviennent probablement de la destruction d'un habitat du . éolithique Récent. Dans la partie centrale de la zone 


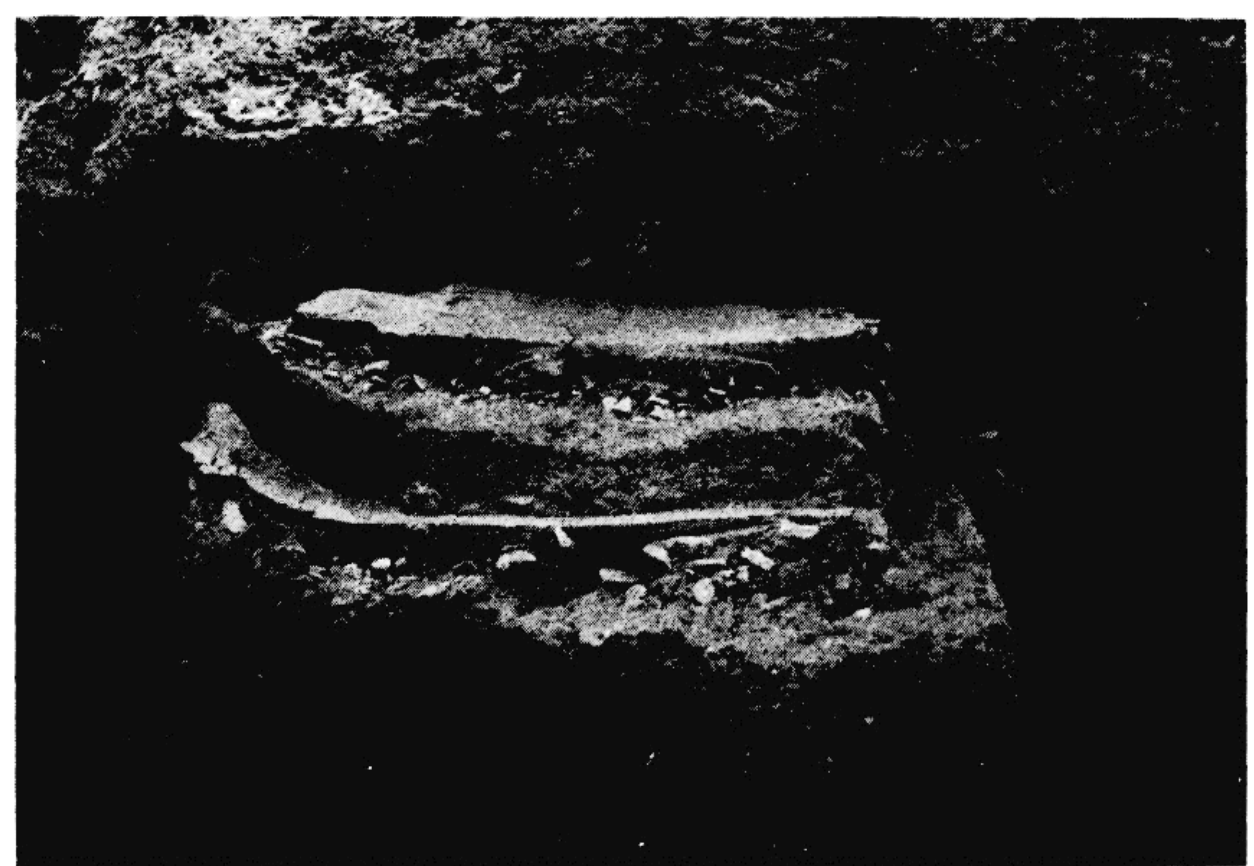

Fig. 2. - Dikili Tash. Secteur I. Le four du Néolithique Moyen en cours de démontage.

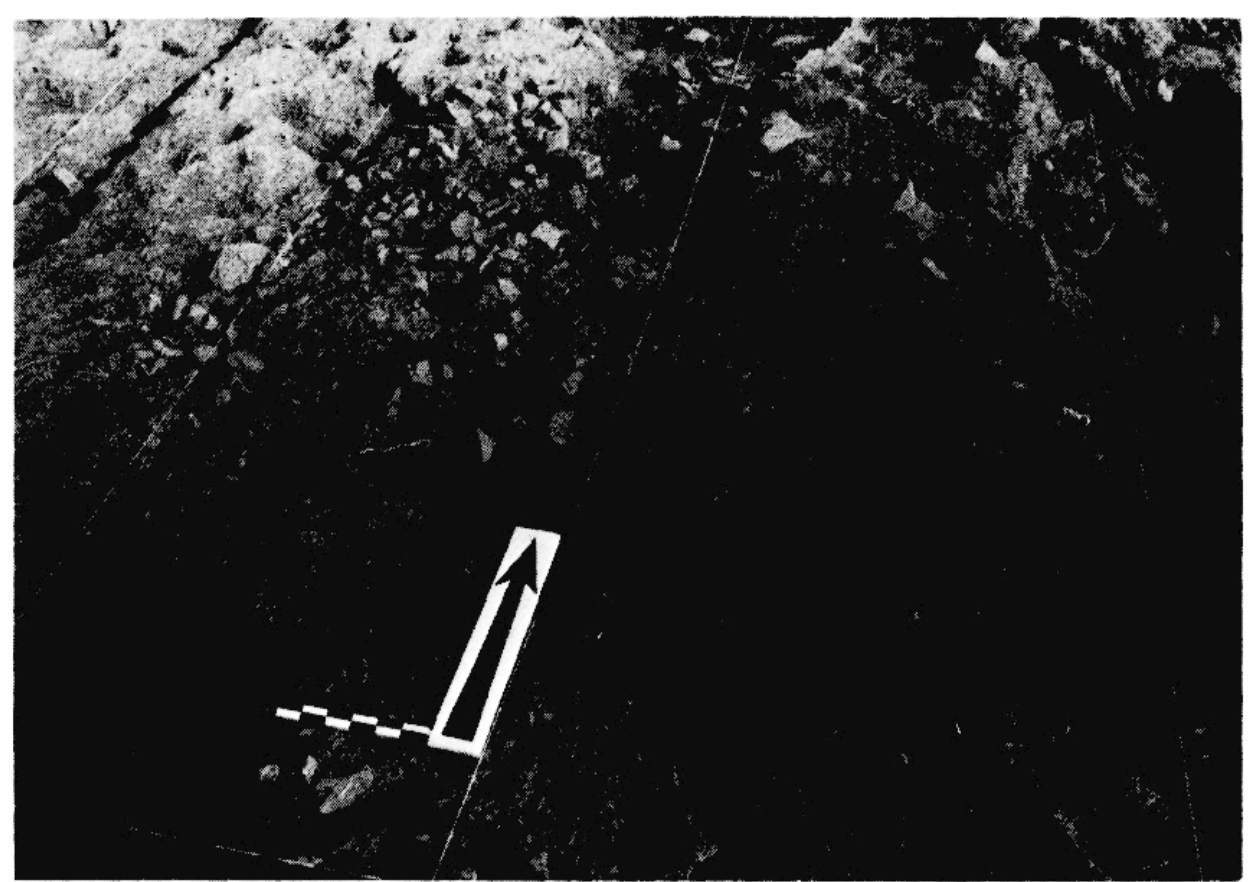

Fig. 3. - Dikili Tash. Secteur V. Sol(?) d'habitation du Néolithique Récent. 


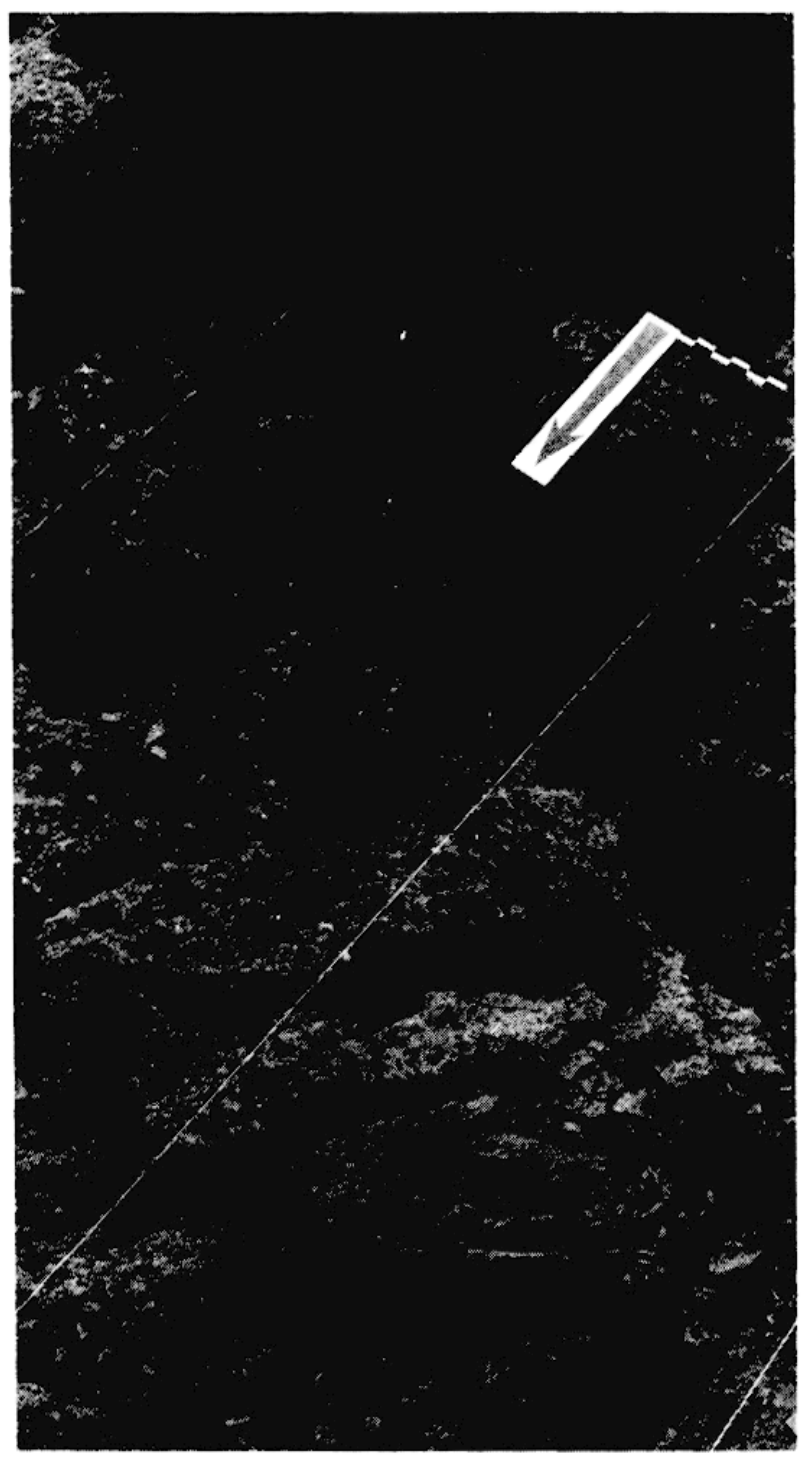

Fig. 4. - Dikili Tash. Secteur V. Le sommet de la couche de destruction du . Véolithique Moyen.

fouillée, en particulier, on a pu dégager un ensemble cohérent de vestiges - vases et tessons, débris alimentaires, pierres - qui correspond probablement au sol d'une maison (fig. 3). Cet ensemble a été démonté après avoir fait l'objet d'un relevé très précis.

Dans la partie Nord-Ouest du secteur $V$, en revanche, la fouille a rencontré, immédiatement sous la surface, le sommet d'une couche d'incendie du. Néolithique Moyen qui contenait des éléments architecturaux écroulés et fortement brülés, mais qui avaient conservé des formes étonnamment fraiches. C'est ainsi qu'il a été possible de reconnaitre plusieurs pans de mur encore en place, avec une partie de leurs enduits, ainsi que des fragments de la couverture, des aménagements intérieurs et du sol (fig. 4). Mais la fouille proprement dite de cette construction a èté réservée pour la prochaine campagne. Il parait probable en tout cas qu'elle appartient à un ensemble d'habitations dont certains éléments ètaient dejà visibles à l'Ouest dans l'ancien carrè AA 28 et dont d'autres le sont maintenant au Nord-Est, près de la limite orientale du secteur V.

Après une année réservée à l'étude du matériel, les buts de la prochaine campagne de fouille seront donc d'une part la poursuite des sondages profonds pour atteindre les couches d'occupation les plus anciennes, d'autre part l'élargissement du secteur $V$, de façon à pouvoir ètudier, en plan, un ensemble d'habitations du Néolithique Moyen : c'était là, en effet, l'objectif principal de la reprise des fouilles à Dikili Tash. 


\section{THASOS}

\section{1. - Champ Théologitis \\ par Jacques Y. Perreault}

L'École Française d'Archéologie a effectué une seconde campagne de fouille dans le champ Théologitis. Celle-ci s'est déroulée en deux temps : du 22 juin au 4 juillet. aver. 12 ouvriers et. l'assistance de Saskia Deluy, stagiaire, puis du 3 au 15 août avec 6 ouvriers, après dépose de la mosaíque. Olivier Picard a identifié les monnaies et les plans ont été relevés par Tony Koželj.

Les objectifs de cette campagne étaient de dégager les angles des trois pièces contiguës mises au jour l'année dernière et d'effectuer des sondages sous les sols pour en préciser la chronologie (fig. 1). En aucun endroit le sol vierge n'a pu être atteint, la hauteur inhabituelle de la nappe phréatique empèchant toute fouille en profondeur.

\section{La pièce Nord-Ouest (fig. 2).}

Il est maintenant clair que la pièce Nord-Ouest et celle qui lui est adjacente ont fait partie, dès l'origine, du mème ensemble architectural.

- Le plus ancien état dègagé, correspondant probablement à la première occupation du bâtiment, remonte à l'époque hellénistique. Durant cette phase, le sol intérieur était légèrement plus bas que le seuil de l'entrée et la présence de crapaudines indique l'existence de deux portes. L'entrée, qui donnait vraisemblablement sur une cour, était flanquee de deux colonnes que l'on a retrouvées couchées le long du parement extérieur.

- A cette phase d'occupation appartient un égout qui longe le parement extérieur du mur . Nord-Ouest de la pièce.

- Ce premier sol était recouvert d'une fine couche de charbon de bois puis d'un remblai d'une dizaine de centimètres d'épaisseur sur lequel reposait le second sol de la pièce. La présence dans ce remblai d'une monnaie datant du règne d'Hadrien permet de situer cette phase dans la première moitié du He siècle ap. J.-C.

- Une autre couche de charbon de bois $(0,10 \mathrm{~m})$ sépare ce sol de la substructure de la mosaïque. La mise en place de celle-ci a été l'occasion d'un important remaniement puisque l'on a procédé à un rehaussement des murs Nord-Ouest, Nord-Est et Sud-Est, réduisant la largeur de l'entrée à une seule porte. Le support du lit de pose de la mosaĩque était constitué d'une couche de pierres disposées obliquement sur l'ensemble de la surface, surmontées d'un rang de pierres plates et de tuiles.

\section{La pièce médiane (fig. 3).}

On a achevé la fouille de la partie Sud-Ouest de cette pièce rectangulaire et effectué un sondage sous le dallage au Centre-Est.

- La fouille sous le dallage a révèlé l'existence de deux sols antérieurs correspondant aux deux premiers états de la pièce voisine. L'entrée, comme l'indique la présence de deux crapaudines in situ, se trouvait alors au Nord-Est, dans le prolongement du seuil de la pièce . Nord-Ouest et donnait done également sur la cour.

- Lors de la mise en place de la mosaĩque, la pièce médiane a également été réaménagée. Le sol fut alors rehaussé par une couche de pierres sur laquelle on posa le dallage. L'entrée . Nord-Est fut murée et une ouverture percée dans le mur Sud-Est. Dans la moitié Ouest de la pièce, on aménagea une canalisation qui longe le mur de fond et tout le parement intérieur de cette partie du mur Vord-Ouest (fig. 4). Un petit bassin rectangulaire en marbre a été trouvé à l'extrémité Sud-Ouest du dallage. Il ne fait aucun doute que cette partie de la pièce servait de latrines, l'apport d'eau se faisant par une ouverture à l'extrémité Est du mur Sud-Est.

\section{La pièce Sud-Est (fig. 5).}

La fonction de cette troisième pièce demeure énigmatique tout comme son extension totale qui n'a pas pu étre dèterminée, notamment parce qu'une tranchée de récupération moderne a fait disparaître une bonne partie des structures. 


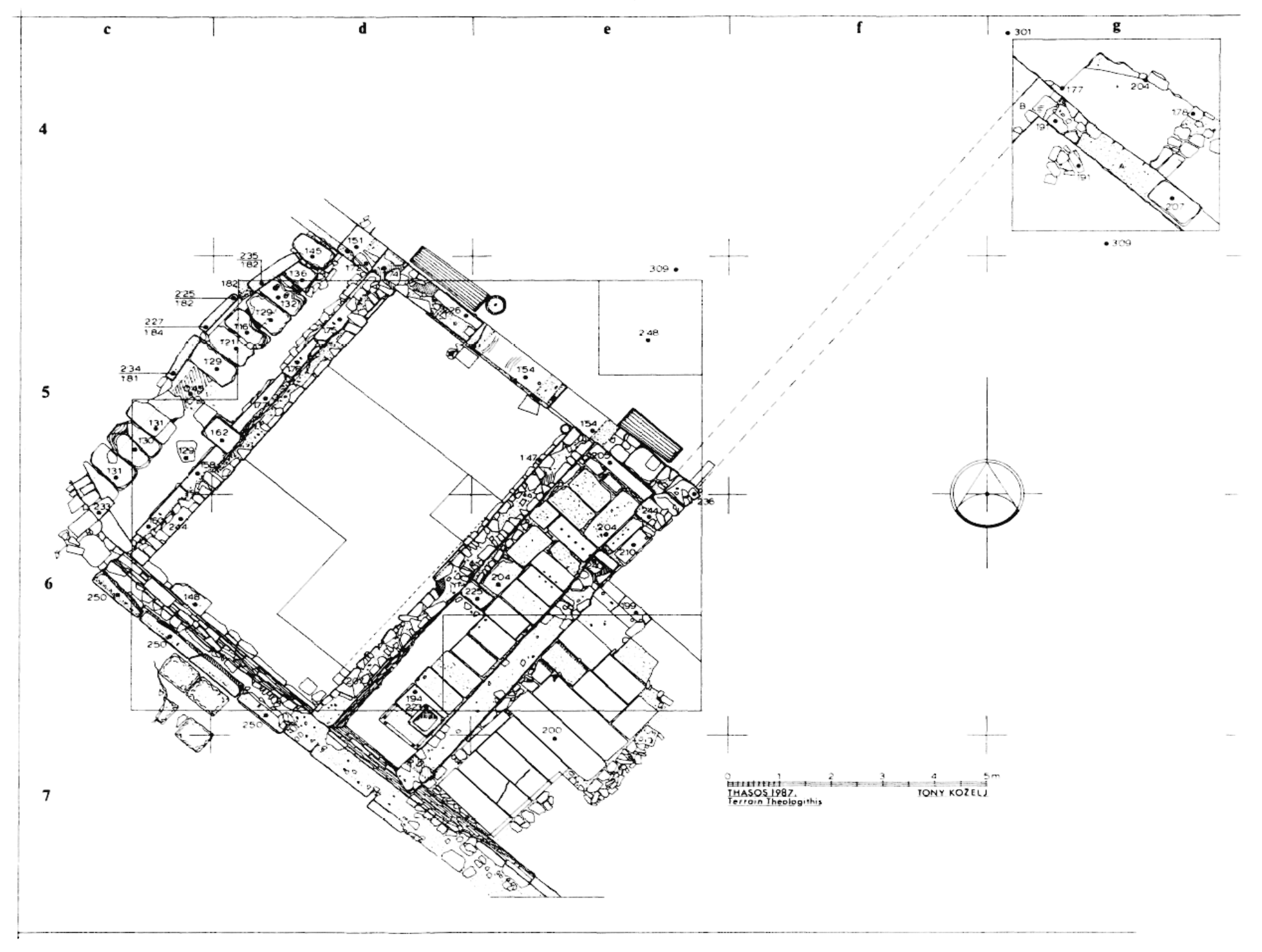

$\underset{8}{\mathscr{8}}$

공

Fig. 1. - Champ Théologitis; plan d'ensemble (T. Koželj). 1:1500. 


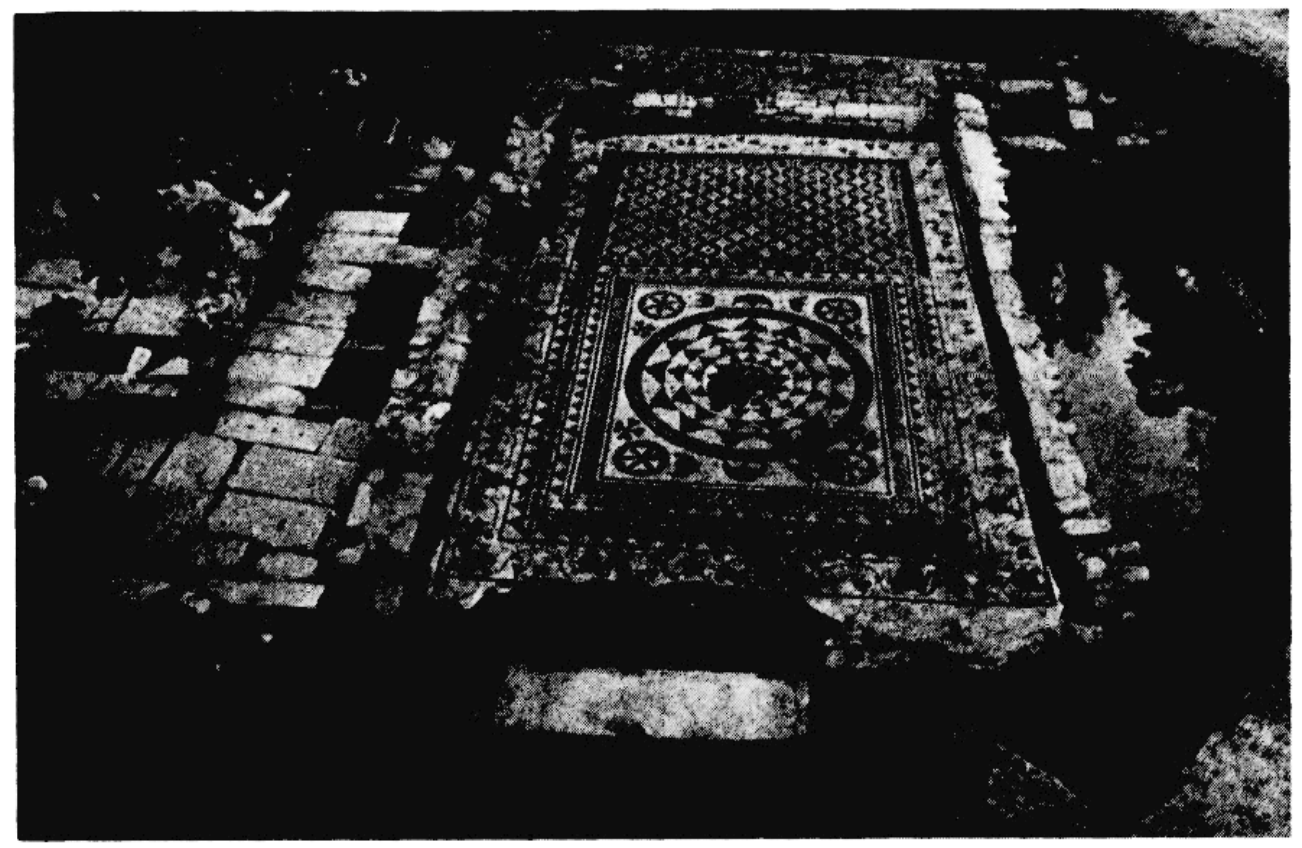

Fig. 2. - La pièce Nord-Ouest avec la mosaïque en place et la pièce médiane, vers l'Ouest.

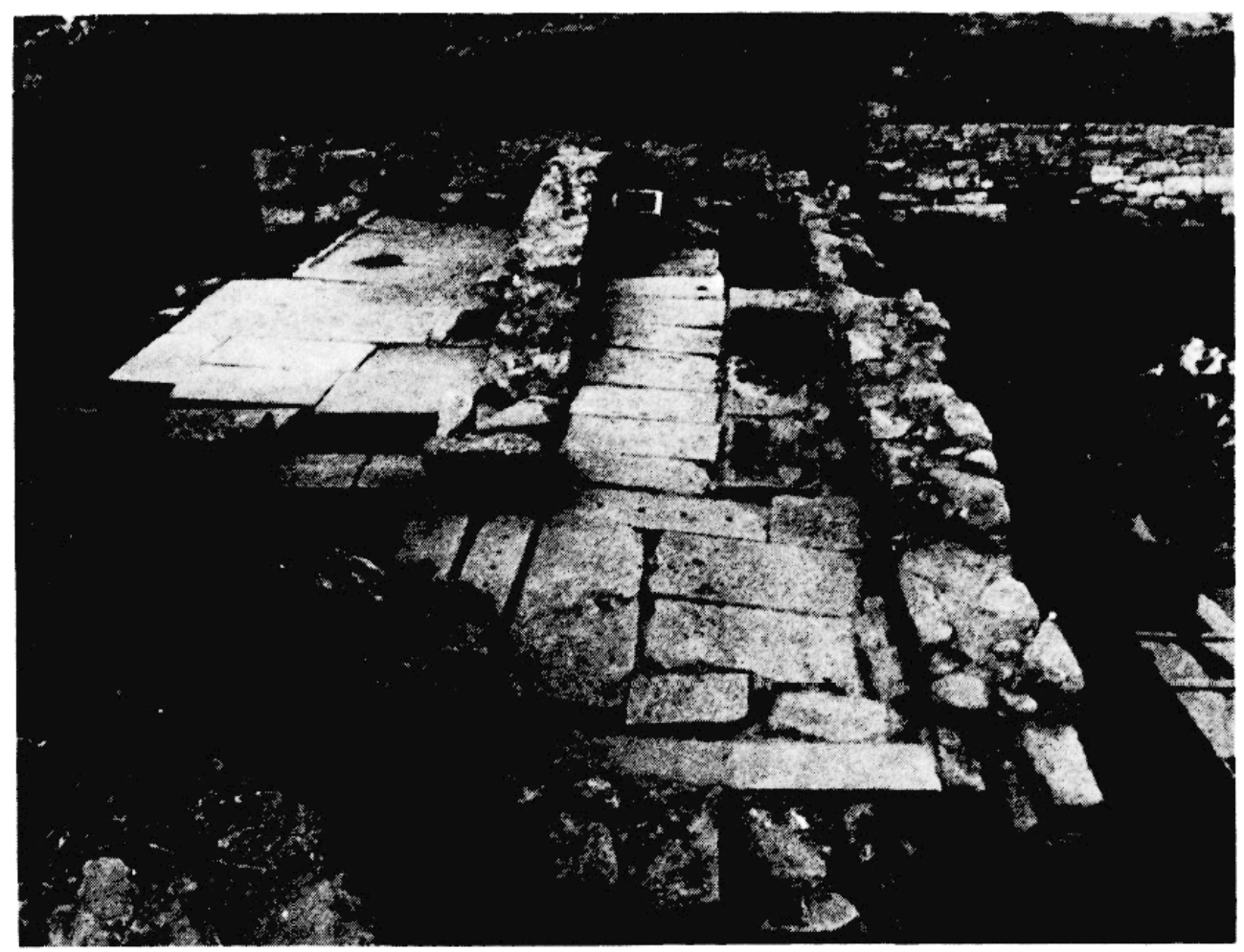

Fig. 3. - La pièce médiane et la pièce Sud-Est, vers l'Ouest. 


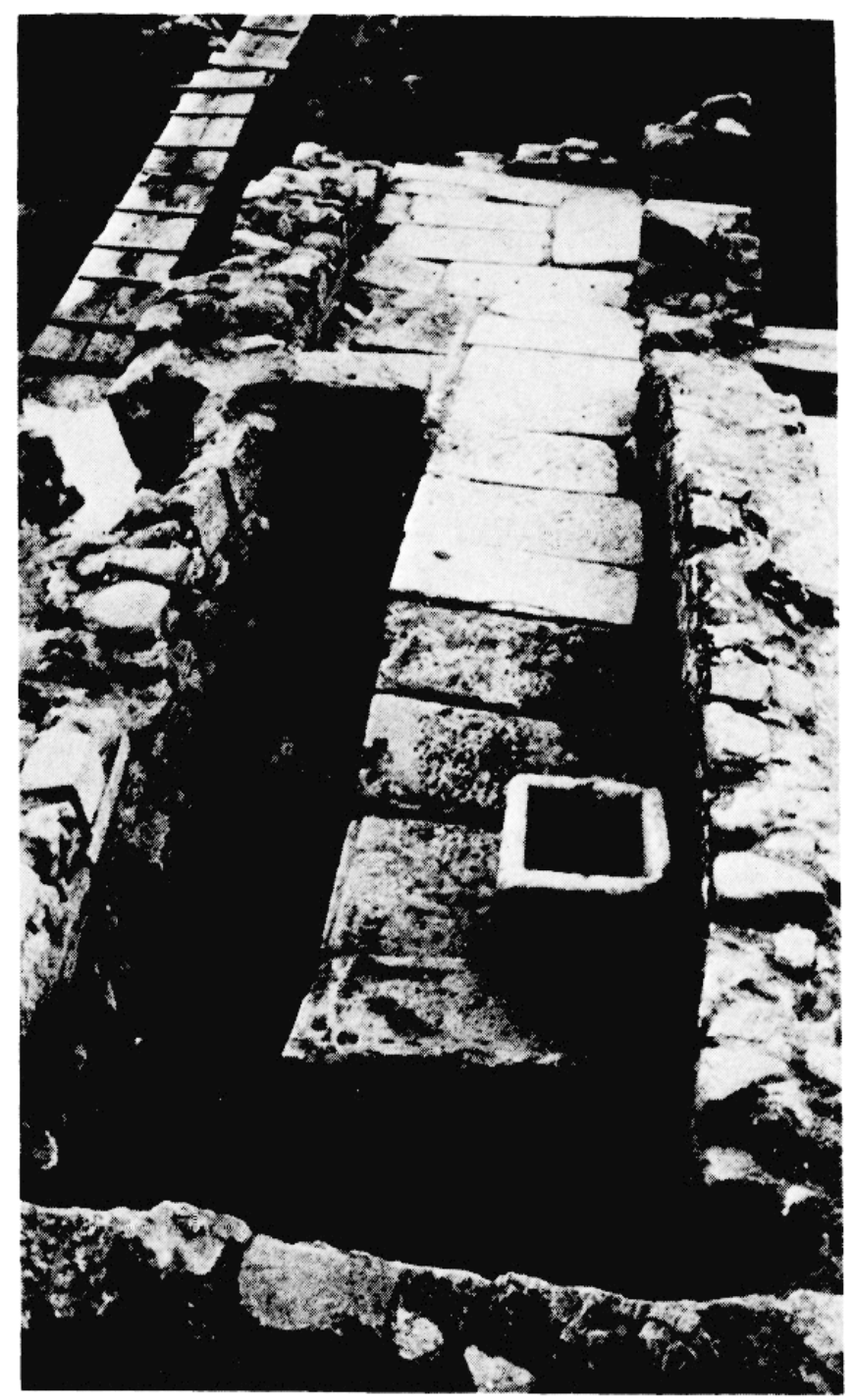

Fig. 4. - Détail des latrines (pièce médiane), vers l'Est.

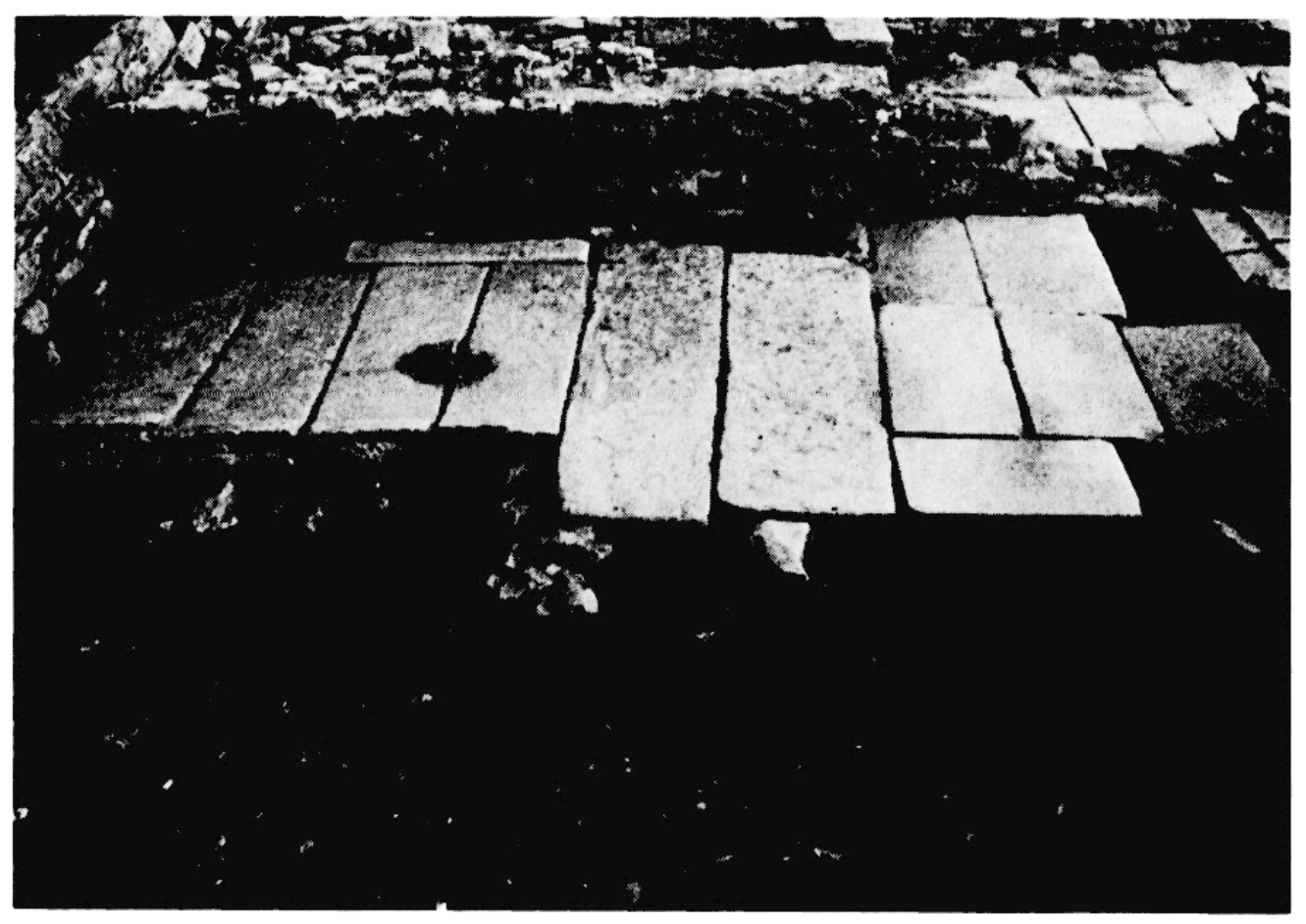

Fig. 5. - Détail du dallage de la pièce Sud-Est, vers le Nord. 
- Seul l'état le plus récent a pu être dégagé. Le sol en est constitué, dans la moitié Sud, de quatre grandes dalles rectangulaires posées perpendiculairement au mur Nord-Ouest. La longueur de chaque dalle est de $1,90 \mathrm{~m}$, sur une largeur totale de $2,30 \mathrm{~m}$, et chacune se termine par un épais rebord. La 7.one au Sud-Fst est constituée d'un sol en terre battue, à une vingtaine de centimètres sous le niveau des dalles, et au Nord-Est d'un dallage de petites plaques rectangulaires. On accédait à cet espace par une entrée au Nord-Est.

\section{Le matériel.}

Mis à part quelques tessons de vernis noir hellénistiques, et la présence de fragments d'époque classique tendant à montrer l'existence dans ce secteur de la ville de niveaux plus anciens, la plus grande partie des trouvailles, d'époque romaine, consiste en céramique commune de qualité médiocre.

\section{Chronologie.}

L'étude de la céramique et des monnaies a permis de situer la date de la destruction finale de ce complexe dans la seconde moitié du ve siècle ap. J.-C., et plus probablement au cours du troisième quart. La mise en place de la mosaĩque a donc dù s'effectuer quelque part entre la première moitiè du $I^{*}$ siècle ap. J.-C. et cette destruction. Il est à espérer que l'étude stylistique permette de réduire cet écart.

\section{2. - Le port}

par Jean-Yves Emprafur et Angeliki Srmossı

Au printemps 1987, du 5 au 29 mai, on a procédé à une troisième campagne de fouilles dans le port antique de Thasos (cf. le rapport sur les deux campagnes précédentes dans le BCH 111 [1987], p. 622-626).

L'équipe ètait dirigée par G. Papathanassopoulos, éphore des Antiquités sous-marines assisté sur le terrain par Angeliki Simossi, pour la partie grecque et par Jean-Yves Empereur pour la partie française. L'équipe ètait composée d'Annick Chẻle, archéologue; de P. Abazopoulos, T. Koželj et N. Lianos, architectes; J.-C. Hurteau,

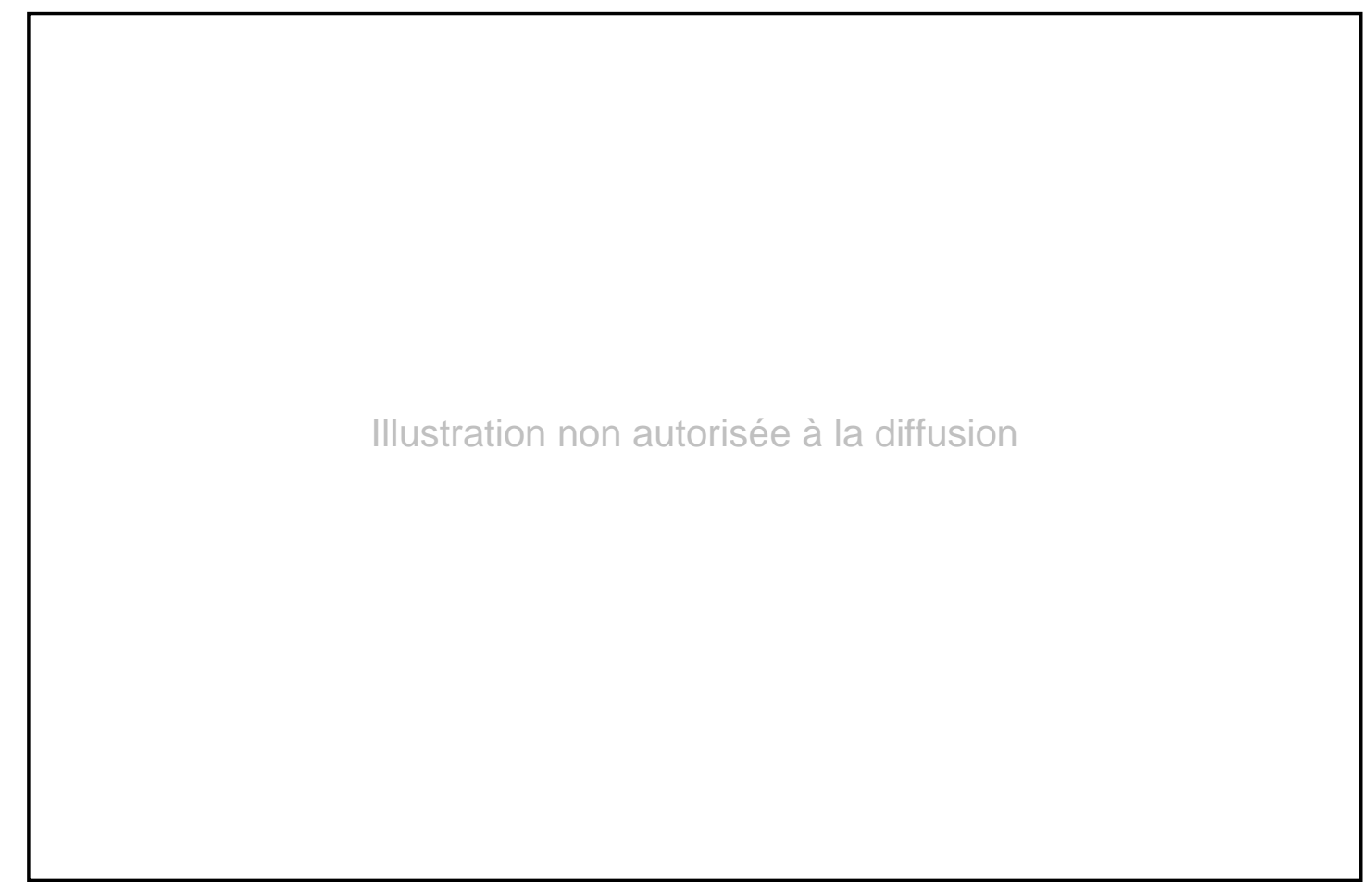

Fig. 6. - Vue du sondage le long de la face intérieure du môle archaïque. Photographie Jean-Claude Hurteau. 


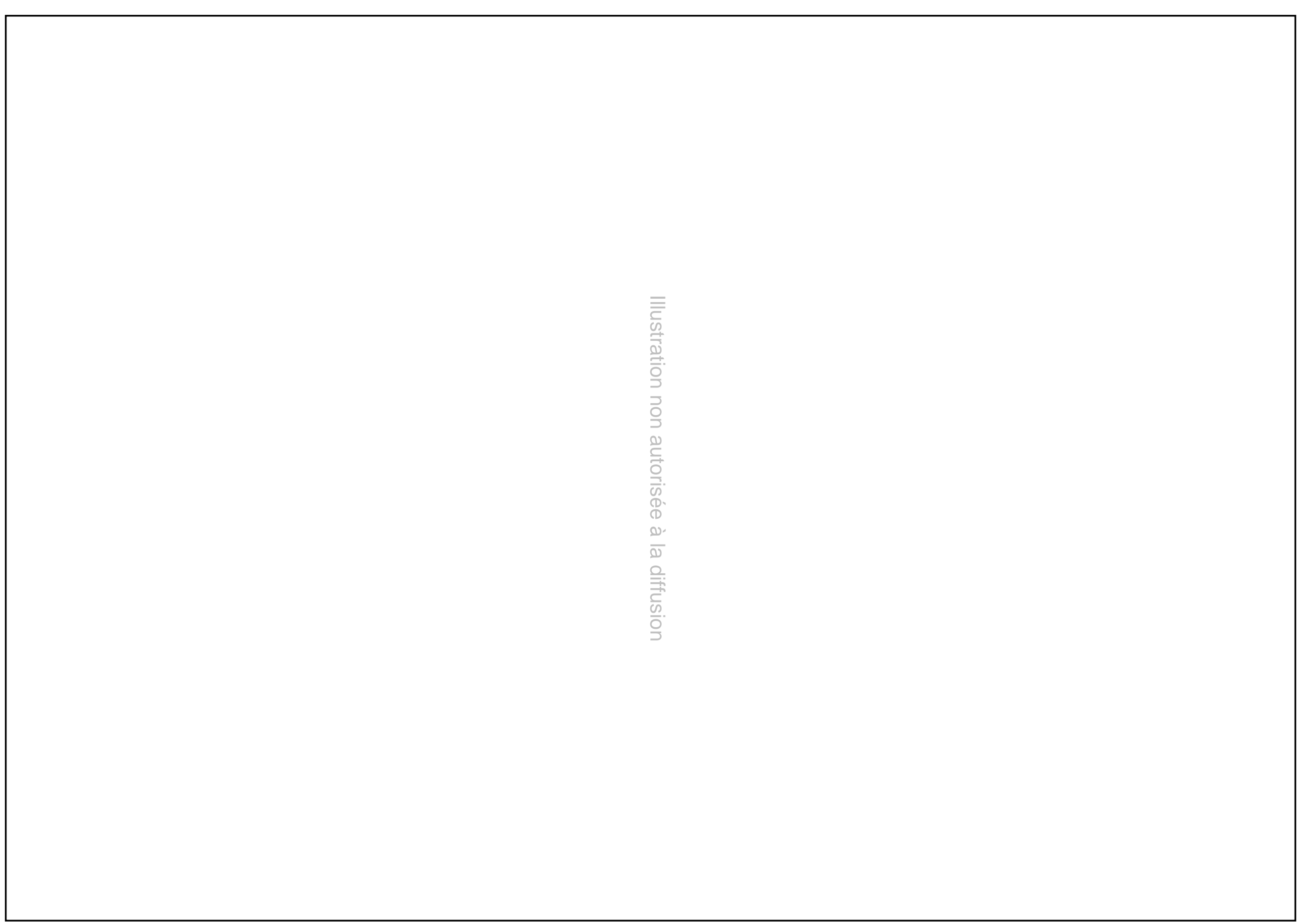

Fig. 7. - Plan du môle archaïque. Dessin Nikos Lianos et Tony Koželj. 

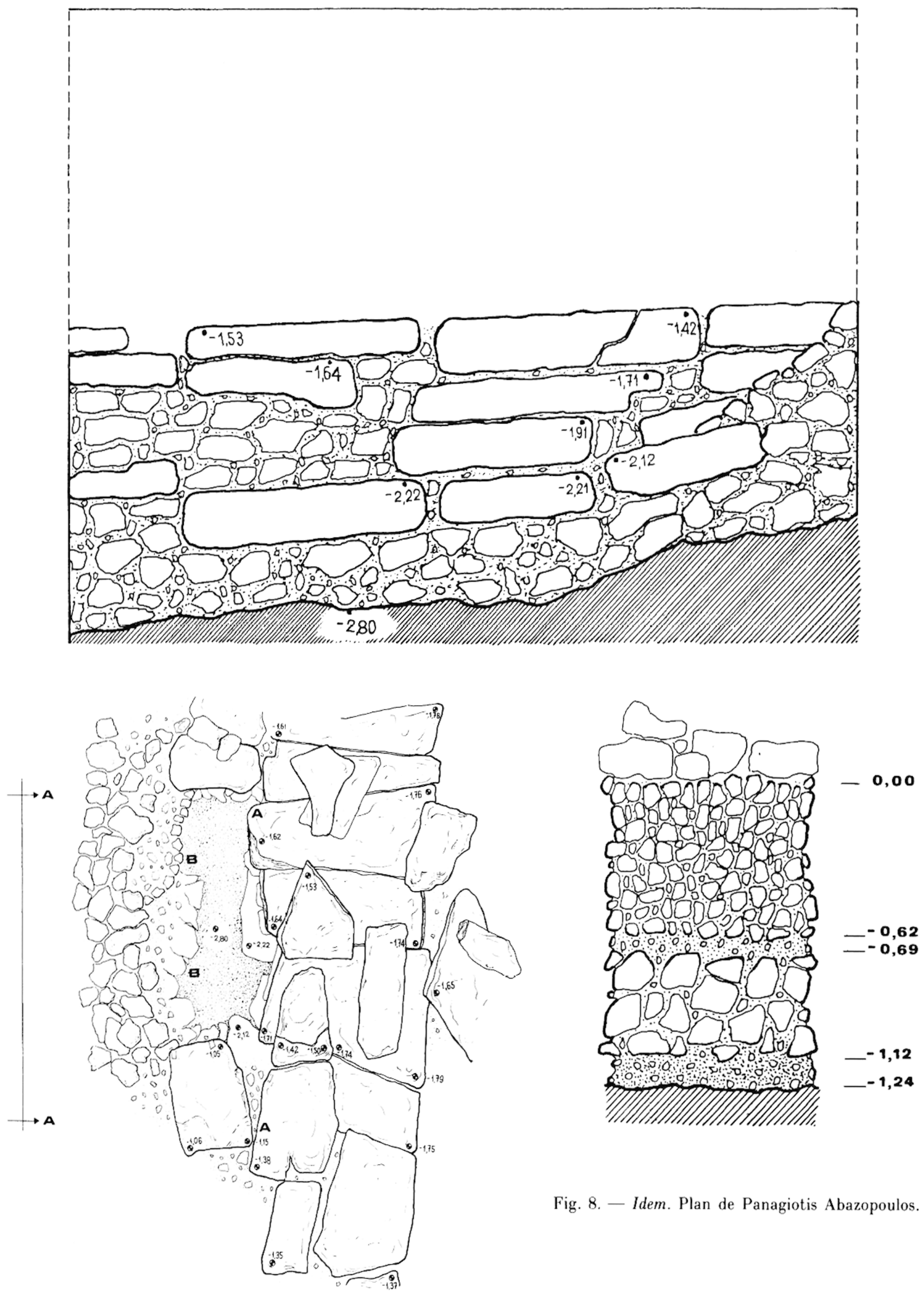

Fig. 8. - Idem. Plan de Panagiotis Abazopoulos. 
Fig. 9. - Vue du sondage à l'extérieur du mỏle archaïque. Photographie Jean-Claude Hurteau.

Fig. 10. - Les plaques de schiste du môle archaĩque. Photographie Jean-Claude Hurteau. 


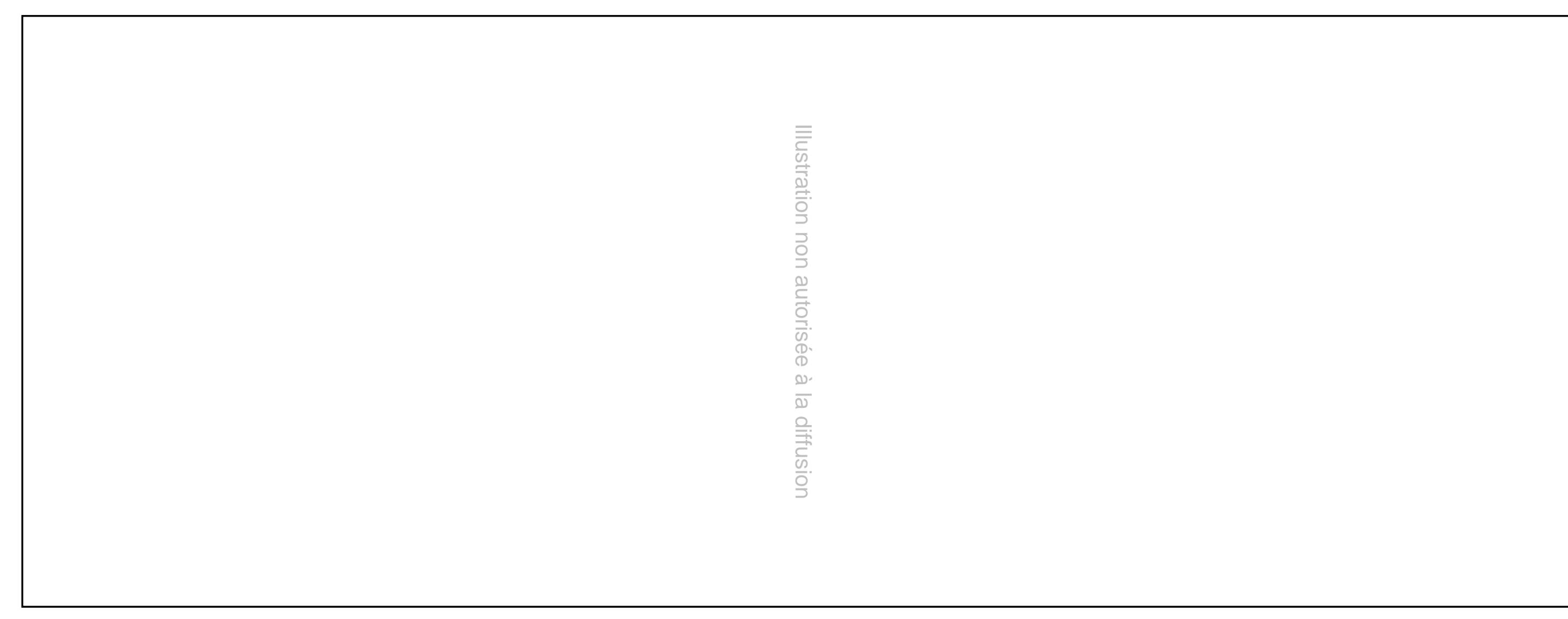

Fig. 11, - Le port classique : partie du môle Nord-Est. Dessin Panagiotis Abazopoulos. 
Fig. 12. - Bloc de marbre avec mortaise de scellement, provenant du môle archaïque. Photographie Jean-Claude Hurteau.

photographe-plongeur du CNRS; P. Antoniou, L. Bochaton et K. Konstantopoulos, plongeurs; C. Aubert, archéologue, assurait l'enregistrement des trouvailles et $\mathrm{S}$. Maninou la restauration. Nous avions à notre disposition quatre pompes à eau qui nous permirent de travailler simultanément à autant d'endroits.

Nous avons choisi de porter nos efforts sur le nettoyage et le relevé du mỏle archaïque découvert en 1985 (fig. 7). Nous avons repris son dégagement là où nous l'avions laissé il y a deux ans. Nous avons pu complèter le tracé jusqu'à son retour vers le quai de béton moderne. Le móle archaíque part donc du Nord-Est de la tour verte d'entrée du port, il tourne vers l'Ouest à $11 \mathrm{~m}$ au large de cette tour puis continue à une distance de $25 \mathrm{~m}$ au Nord-Ouest de tour no 3 dégagée en 1984 puis il s'oriente vers le large sur une longueur de 50 m, s'éloignant jusqu'à un maximum de $58 \mathrm{~m}$ du quai moderne vers lequel il revient bientôt après un angle arrondi marqué par la présence de trois forts orthostates. Depuis son point de retour vers le quai moderne jusqu'à la tour d'entrée du port nous mesurons une distance de plus de $120 \mathrm{~m}$.

En deux endroits nous avons entrepris d'étudier l'élévation du môle antique. A cette fin, nous avons ouvert deux sondages triangulaires, le premier le long de la face intérieure du mỏle et le second à peu près à la méme hauteur le long de la face extérieure.

Dans ce dernier sondage d'une largeur de $5 \mathrm{~m}$ nous avons dégagé quatre assises de blocs de schiste sur une hauteur de plus d'1 $\mathrm{m}$ (fig. 7 no 15 et fig. 9).

Sous une couche de sable et de graviers qui se poursuit jusqu'à la troisième assise, nous avons trouvé une épaisse couche remplie d'abondante céramique, notamment des fragments d'amphores thasiennes.

Les rares indications chronologiques nous ramènent au plus tôt vers la fin du ive siècle avant notre ère telle une anse timbrée au nom de Pouliadès. Les autres trouvailles sont plus récentes, telle une tuile de Satyros ( $1^{\text {re }}$ moitié du II $^{\circ}$ siècle avant notre ère) (fig. 8) ou une monnaie de bronze à l'lléraclès archer et Artémis au Ier siècle avant notre ère (fig. 9) (cf. Guide de Thasos, p. 189 et planche V).

Le sondage pratiqué à l'intérieur du môle a permis de le dégager sur une longueur de $3,50 \mathrm{~m}$ (voir fig. 7 $n^{\circ} 12$ et fig. 6). La dernière assise supérieure conservée se trouve à $1,10 \mathrm{~m}$ sous le niveau actuel de la mer. Nous avons mis au jour 5 assises jusqu'à la cote $-2,60$ (fig. 8) traversant une épaisse couche de grès de plage (beach rock) $(0,62 \mathrm{~m})$ suivi d'une couche de graviers $(0,07 \mathrm{~m})$ puis de grosses pierres $(0,43 \mathrm{~m})$. Sous le niveau de la première assise du mỏle, une couche de graviers et de sable $(0,12 \mathrm{~m})$ repose directement sur la vase vierge.

Le grès de plage, particulièrement dur à traverser emprisonnait de nombreux fragments céramiques principalement d'amphores thasiennes. 
Parmi les sept anses timbrées, nous n'avons pu déchiffrer que celle d'Aristodicos au Kados que M. Debidour (BCH Supplément XIII [1986], p. 331) date de la fin du Ive siècle avant notre ère. Cette couche donne la date de la destruction du môle archaïque.

Nous n'avons que peu d'indices sur l'époque où il fut bâti. Les seuls élèments chronologiques sont fournis par son mode de construction (grandes dalles de schiste alternant avec des cubes de marbre) et notamment les scellements en double queue-d'aronde (fig. 12) qui le rendent comparable à d'autres monuments de Thasos datés de la fin du vie siècle avant notre ère ou du début du ve siècle comme à l'Héracléion ou avec le puits circulaire du terrain Valma (cf. $B C H 109$ [1985], p. 876).

L'inclinaison des assises du mỏle archaĩque vers le large (environ $45^{\circ}$ ) montre que le port a été détruit à la suite d'un phénomène naturel, séisme ou glissement de terrain (fig. 10).

Les sondages de 1984 et 1985 ont permis de dater la construction du port fermé de l'extrême fin du Ive siècle avant notre ère notamment par un sondage en sape sous la muraille Ouest (cf. $B C H$ 111 [1987]). Cela signifie que la construction du port fermé a été engagée aussitôt après la destruction du port archaique.

Les architectes du ive siècle ont préféré construire un port plus petit sur des bases plus stables, avec un jeu d'angles rentrants fort différents des courbes du monument archaïque.

Mis à part le relevé d'une partie du môle Est par $P$. Abazopoulos nous n'avons pas eu les moyens cette année de continuer nos recherches sur le port fermé (fig. 11). Il est prévu de les reprendre en 1988, notamment avec le dégagement d'une nouvelle tour au Nord-Est et de suivre le tracé de l'ensemble du móle Est, afin de compléter la nouvelle image que nous obtenons peu à peu de ce port.

\title{
3. - Nettoyage sur la tour située d'Ouest de la porte d'Héracles et de Dionysos
}

\author{
par Yves Grandjean, Haïdo Koukouli-Chryssanthaki, Tony Kožel.J, François Salviat
}

En 1977, des recherches engagées sur un programme de collaboration entre le Service archéologique grec et l'École française avaient été effectuées sur l'enceinte dans la région de la plaine. Flles avaient porté en particulier sur la tour située à l'Ouest de la porte d'Héraclès et de Dionysos (cf. Y. Grandjean, H. Koukoult et R. MARTin, BCH 102 [1978], p. 811-813). Le terrain voisin étant propriété privée, il n'avait été possible que de pratiquer des sondages limités, l'un à l'intérieur, l'autre à l'extérieur de la tour. On avait dù alors laisser sur place un amoncellement de blocs, marbre et gneiss mêlés, visible à l'intérieur de l'édifice dont l'intérêt avait été signalé.

En 1987, le terrain ayant été acquis par l'État grec, on a pu procéder (toujours en collaboration avec l'éphorie) au dégagement et au classement de ces blocs. On a ainsi constaté que les pièces architecturales tombées à l'intérieur de la tour reposaient sur un remblai mis en place lorsque les deux portes basses furent murées (II ${ }^{e}$ s. av. J.-C. au plus tôt; cf. les trouvailles de monnaies dans le sondage intérieur : $B C H$ [1978], p. 812).

En 1977, on avait recensé seulement quatre blocs, taillés en chaperon, parmi lesquels deux étaient complets, dont une pièce d'angle (fig. 13, 14); ceux-ci permettaient de reconnaître l'existence de créneaux en partie haute. Grâce à la découverte, en 1987, de nombreux éléments de marbre provenant du parapet de couronnement, l'élévation de la partie haute de la tour peut ètre désormais restituée.

Le parapet (epalxis), constitué de parpaings de marbre piqués sur les deux faces, épais de $0,48 \mathrm{~m}$ en moyenne, comportait un mur plein, sur une hauteur qu'on peut évaluer à quatre assises. Au niveau du lit supérieur de la quatrième assise (haute de $0,26 \mathrm{~m}$ ) commence une alternance de merlons larges $(1,75 \mathrm{~m}$ à $1,80 \mathrm{~m}$ et $1,80 \mathrm{~m}$ à l'angle au niveau du couronnement) et d'embrasures étroites ( $0,70 \mathrm{~m}$ environ). On compte en façade, pour une largeur totale de l'ordre de $9,50 \mathrm{~m}$, quatre merlons (dont deux en position angulaire) et trois embrasures, dont une en situation axiale. Il est certain - le nombre des blocs nous en assure - que le dispositif faisait retour sur les côtès de la tour, très vraisemblablement dans le mème rythme, la tour étant à ce niveau de plan carré.

La hauteur des "merlons" est d'environ $1 \mathrm{~m}$. Chacun d'eux est couronné par une pièce monolithe légèrement débordante à profil de chaperon (en pyramide tronquée au-dessus d'une partie parallélépipédique). Au-dessous, deux blocs superposés, également monolithes, tous les deux hauts de $0,33 \mathrm{~m}$. Ces blocs étaient liès verticalement entre eux, au chaperon, et à l'assise inférieure de margelle, par des gou ns verticaux scellès à découvert sur leurs deux faces latérales (plus bas, le parapet est assemblé sans liaisons métalliques).

Les embrasures montrent à l'appui la mème section que le couronnement des merlons (un sommet plat et un double biseau). Les blocs, généralement jointoyés (à une exception près) vers le milieu des ouvertures, 


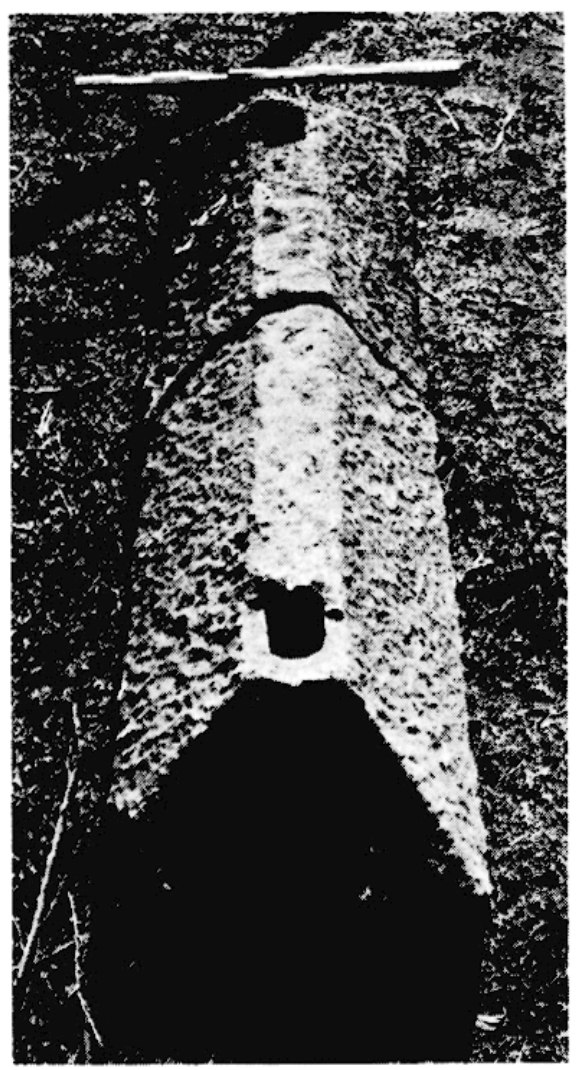

Fig. 13. - Chaperon courant.

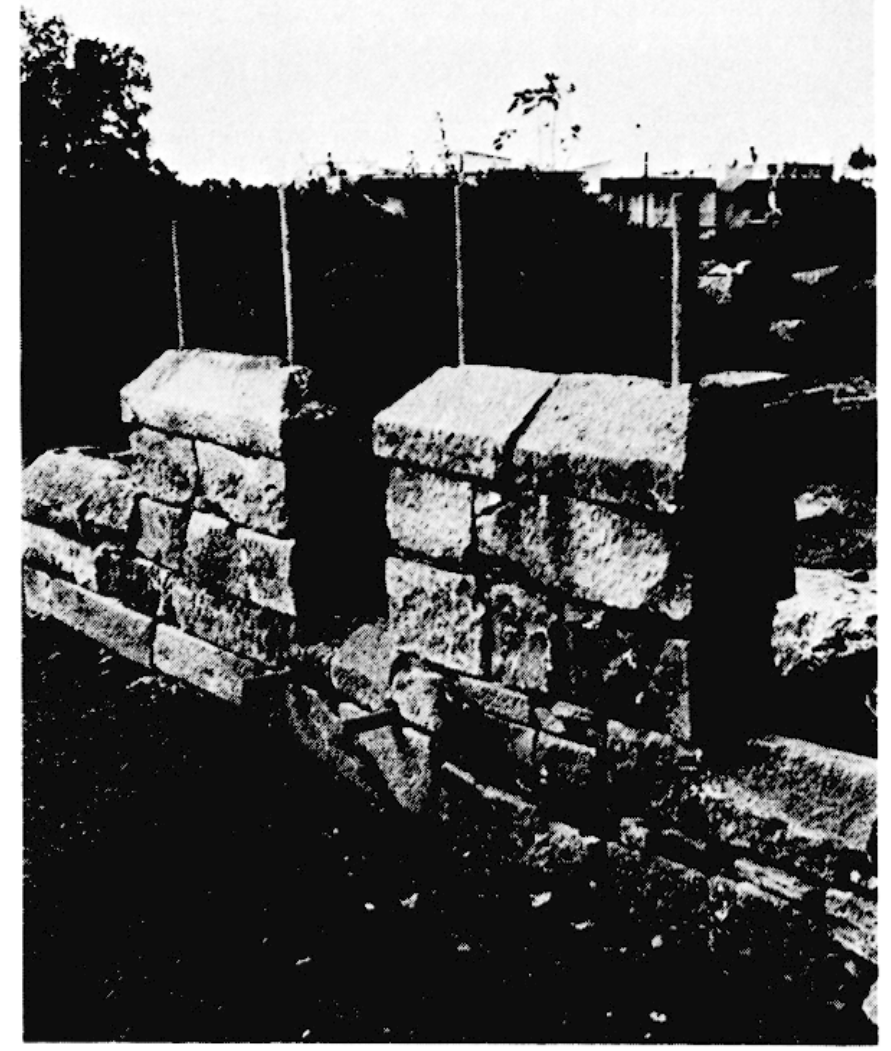

Fig. 16. - Reconstitution partielle du parapet crénelé.

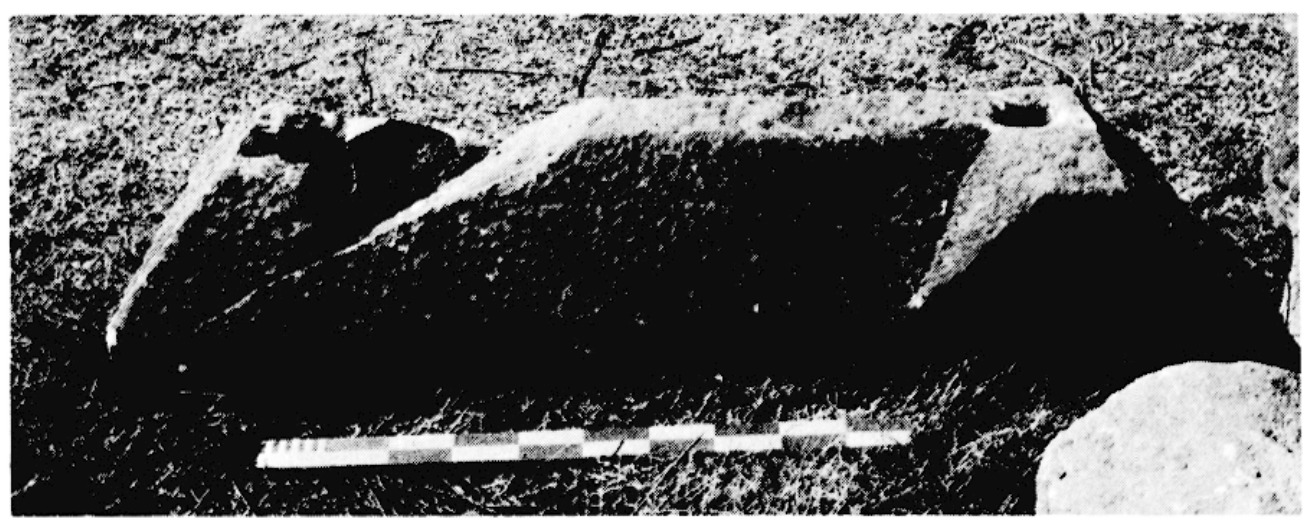

Fig. 14. - Chaperon d'angle.

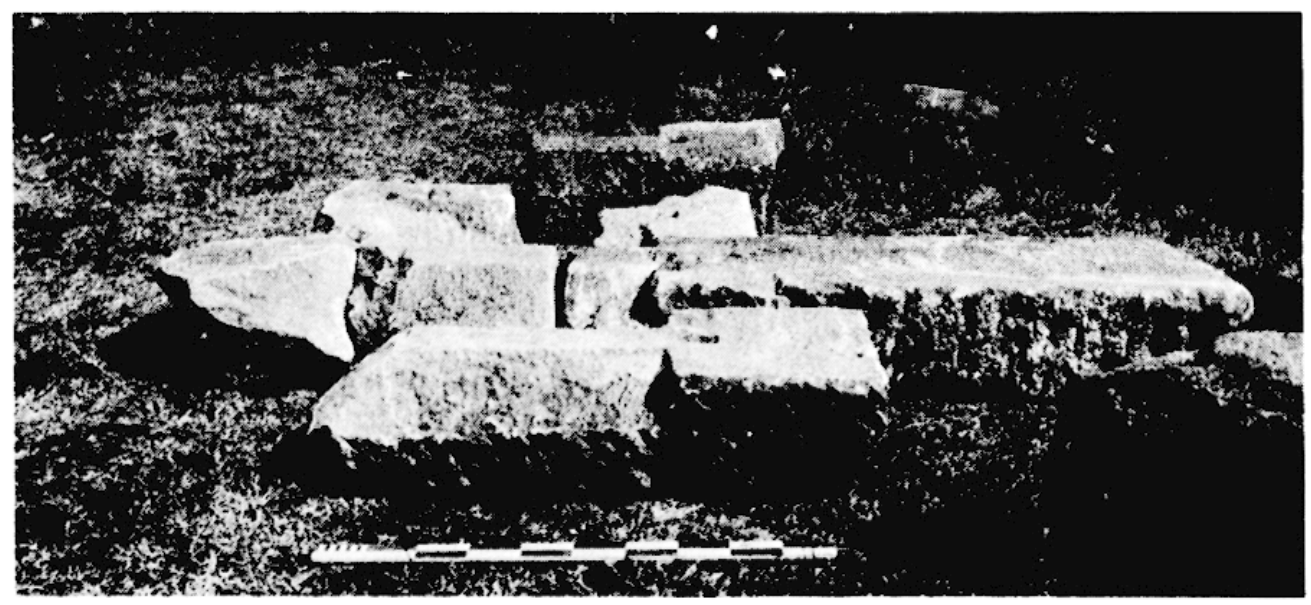

Fig. 15. - Assises de margelle. 
Fig. 17. - Dessin du parapet (reconstitution). Dessin T. Koželj.

pénètrent en queue sous les merlons et gardent là une section rectangulaire normale. Ils présentent, à découvert, des encoches pour la pince, destincécs à assurcr la misc en place des blocs de baze des merlons.

$\mathrm{Ce}$ parapet crénelé offre en outre une particularité très intéressante : on y observe des mortaises et perforations, très régulièrement disposées, qui affectent l'assise de margelle et la partie haute du merlon.

1. - L'assise de margelle est percée de part en part, à peu près à l'aplomb des faces latérales de chaque merlon, mais légèrement en dehors de l'embrasure. Ces perforations, d'une largeur de 0,10 à $0,11 \mathrm{~m}$, d'une hauteur de 0,07 à $0,11 \mathrm{~m}$, ont été pratiquées à la partie inférieure de l'assise de margelle, avant la mise en place des blocs concernés, par creusement, à la pointe, d'un canal en $\mathrm{U}$ au lit de pose.

2. - Chaque chaperon de merlon est percé verticalement, à chaque extrémité des faces planes supérieures, et de plus, pour le merlon d'angle au point de retour, d'une mortaise à section à peu près carrée (dimension des côtés variable de 0,08 à $0,10 \mathrm{~m}$ ) s'achevant au lit d'attente du bloc immédiatement inférieur par une cuvette peu profonde $(0,03$ à $0,05 \mathrm{~m})$. Là encore il s'agit d'un aménagement primitif, ces cavités ayant été pratiquées avant montage; l'examen du travail le prouve. 
Les perforations horizontales ne sont pas sans précédent: on trouve des parapets ou des chaperons qui présentent cette particularité sur d'autres sites, dans des fortifications de mème époque que la tour thasienne (voir par exemple KIEnast, Samos XV, pl. 35,2). On peut interpréter ce dispositif encadrant l'embrasure comme des coulisses destinées à accueillir des pièces de bois, de section ronde, pour écarter ou rapprocher du parapet le bas des volets protégeant l'embrasure. Les mortaises verticales transperçant les chaperons sont plus difficiles à expliquer. Elles semblent propres à recevoir l'extrémité de pièces de bois de section carrée, de la grosseur à peu près de nos chevrons de charpente, régulièrement espacées, au nombre de huit en façade, angles compris. Celles-ci ont pu supporter un abri supplémentaire, tablier vertical de planches, auvent ou toit léger. II paraît a priori assez difficile de les mettre en rapport avec l'attache haute des volets. On observe toutefois, lorsque l'état de conservation des marbres le permet, que le bord de la mortaise est partiellement repris, travaillé et aplani : cette particularité s'expliquerait-elle par l'existence d'un système horizontal sur lequel s'articulerait le volet? On ne relève en effet, sur les còtés de l'embrasure, aucune autre trace d'accrochage.

Quoi qu'il en soit de ce problème, on peut considérer que le parapet de cette tour est maintenant connu avec une exceptionnelle précision. Or cette tour fait partie d'un programme qui a concerné toute la fortification

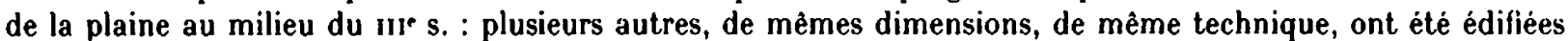
contre le rempart au même moment (cf. Y. Grandjean, Recherches sur l'habitat thasien, ElThas XII [1988], p. 233-235). Ce système de parapet et de créneaux leur est donc sûrement commun. On en est assuré par la tour voisine, située à $40 \mathrm{~m}$ à l'Est de la porte d'Héraclès, large de $9,40 \mathrm{~m}$ en façade, près de laquelle on a trouvé plusieurs blocs de chaperon de merlons, dont un bloc complet, et un bloc d'angle, l'un et l'autre avec mortaises verticales. Il est vraisemblable que les tours près de la porte du Silène, et la tour à l'Est de la porte de Zeus, étaient aussi couronnées de la même manière. L' "avant-corps" de la porte de Zeus lui-mème (une véritable tour d'au moins $10 \mathrm{~m}$ de haut) doit avoir été doté d'un semblable système défensif de la partie haute : un fragment caractéristique de bloc d'embrasure, à chaperon avec la coulisse horizontale pour réglage du volet, y a été en effet recueilli.

Gráce à cet examen nouveau des débris de la tour à l'Ouest de la porte d'Héraclès et de Dionysos, l'image de la fortification thasienne hellénistique se fait donc plus claire. Hors de Thasos, les exemples sont très rares où l'on parvienne à reconstituer aussi sûrement les parties hautes crénelées des ouvrages de défense.

Les sondages et le dégagement partiel de 1977 n'avaient permis de reconnaitre la partie basse des murs de la tour que sur le côté Ouest, dans la région de la porte extérieure et de l'angle avec le parement externe du rempart, et, superficiellement, dans la région de l'angle Sud-Ouest. Le nettoyage rapide de la façade montre que l'assise de lourds orthostates (hauts de 1,04 m) observable à l'Ouest au-dessus de l'euthyntéria de gneiss se poursuivait en façade, avec des blocs de plus grande taille. Les deux orthostates de la partie médiane de la façade sont conservés, en position de dévers vers l'avant; on aperçoit leur lit d'attente. Au-dessus, deux blocs, fort abîmés, de l'assise de parpaings de marbre déjà reconnue à l'Ouest. Il semble que le mur de façade ètait plus puissant que les murs latéraux. Le mur Est enfin a été dégagé sur son parement interne : celui-ci est d'un appareil assez médiocre (gneiss et marbre remployés). Le parement externe de ce mur, inclus dans une propriété privée, reste inconnu. En dépit de cette lacune, le nettoyage de 1987 confirme que la construction de cette tour est très apparentée à celle des tours du mème programme (tour du Silène en particulier) élevées sur le circuit Sud de l'enceinte. Toutes sont des tours creuses, de mème module (à l'exception de la tour du Silène, plus ètoite), qui possédaient une porte vers la ville ménagée dans l'enceinte et une porte latérale extérieure à l'Ouest, toutes deux situées au "rez-de-chaussée", des fenètres ou des meurtrières de tir sur deux niveaux couverts, une partie haute chevauchant partiellement la courtine du rempart tandis que la partie inférieure était plaquée contre le parement externe de la muraille, et un couronnement crénelé à une douzaine de mètres de hauteur.

\section{4. - Phari}

L'Éphorie de Kavala et l'École Française d'Athènes ont poursuivi cette année la fouille de l'atelier de potier d'époque archaĩque situé sur le site de Phari, au Sud-Ouest de l'île. La campagne s'est déroulèe du 22 juin au 4 juillet sous la direction de Catherine Péristéri, Francine Blondé, Jacques Y. Perreault et la participation

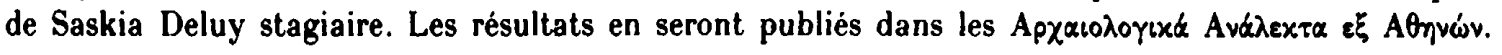




\title{
5. - Carte arch6ologique
}

\author{
par Michèle Brunet
}

L'équipe pluridisciplinaire franco-grecque chargée de l'établissement de la carte archéologique de Thasos a poursuivi son travail au printemps et durant l'été 1987. Des compléments de relevés ont été effectués sur certains sites de la région d'Astris par L. Thomas, architecte; le quart Nord-Ouest de l'île (communes de Rachoni et de Prinos), secteur peu expluré par les voyageurs du début du siècle, a fait l'objet de la campagne de prospection. On accorda une attention toute particulière aux vestiges du réseau routier antique dont de nombreuses traces subsistent dans la région; le schéma de l'implantation humaine, dans les larges vallées de Rachoni et de Prinos s'évasant en plaines côtières, se révèle différent de celui constaté dans le Sud ou aux abords de la cité antique. L'île se présente donc bien comme un ensemble de micro-régions fortement individualisées tant du point de vue géographique qu'économique et humain.

\section{DÉLOS}

\section{1. - Le prytance}

\section{A. Sondages}

par Roland Étienne et Alexandre Farnoux

R. Etienne et A. Farnoux ont mené une campagne de sondages au Prytanée (GD22) avec une équipe d'étudiants de l'Université de Lyon', du 15 juillet au 15 aout. Elle avait pour but de poursuivre les recherches entamées en 1974, 1975 et 1977 et reprises en 1986², avec les objectifs suivants : tout d'abord achever l'étude du site du Prytanée en implantant une série de sondages sur un axe Nord-Sud (fig. 1) jusqu'à l'Agora des Déliens $(G D 84)$ (sondages 19-190, 22, 23 et 26) et sur un axe Est-Ouest (sondages 24 et 25) et ainsi faire l'histoire de cette zone peu bâtie du téménos d'Apollon; ensuite dégager de nouvelles fosses mycéniennes, comme celles trouvées lors des dernières campagnes à l'intérieur du Prytanée et du Bâtiment $\Delta$, pour tenter d'établir les raisons de leur présence dans ce secteur où aucune construction mycénienne n'a été signalée jusqu'à présent ${ }^{3}$; enfin rassembler de nouvelles données sur la préhistoire du sanctuaire d'Apollon.

A l'extérieur du Prytanée, le rocher affleure partout sous une mince couche d'humus (sondages 26 et 23) sauf en 19-190 où une profonde dépression naturelle dans le granit nous a permis d'obtenir une coupe stratigraphique (fig. 2) où l'on distingue un épais remblai argileux sur lequel repose la fondation du portique de façade du Prytanée et qui est posé lui-méme sur une couche de pierres et de terre mélée de tessons mycéniens, d'os et de coquillages comblant le fond de la dépression. En 24 et 25 la situation est moins claire : sur le rocher qui apparaît ici à moins d'un mètre de la surface, les couches ont été très perturbées par l'installation de la grande fondation en poros à l'Est du Prytanée. En aucun endroit sondé nous n'avons trouvé de constructions antérieures à l'édification du Prytanée.

(1) L'équipe était composée de M. Polychronaki, F. Perez, E. Raptou, J.-C. Mossière et A. Rabot.

(2) $B C H 111$ (1987), p. 628-629.

(3) H. Gallet de Santerne, Délos Primitive el archaïque, p. 73. 


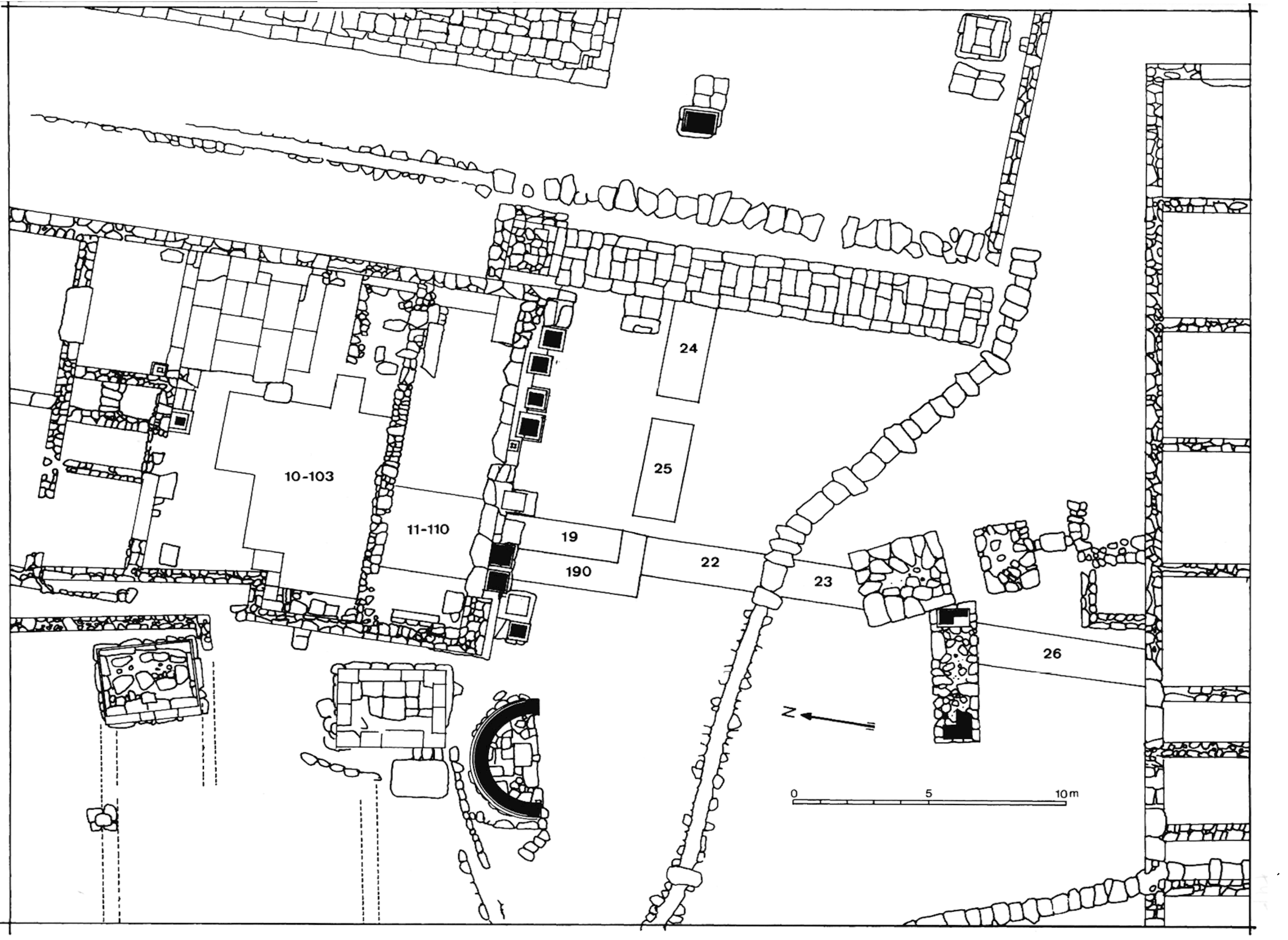




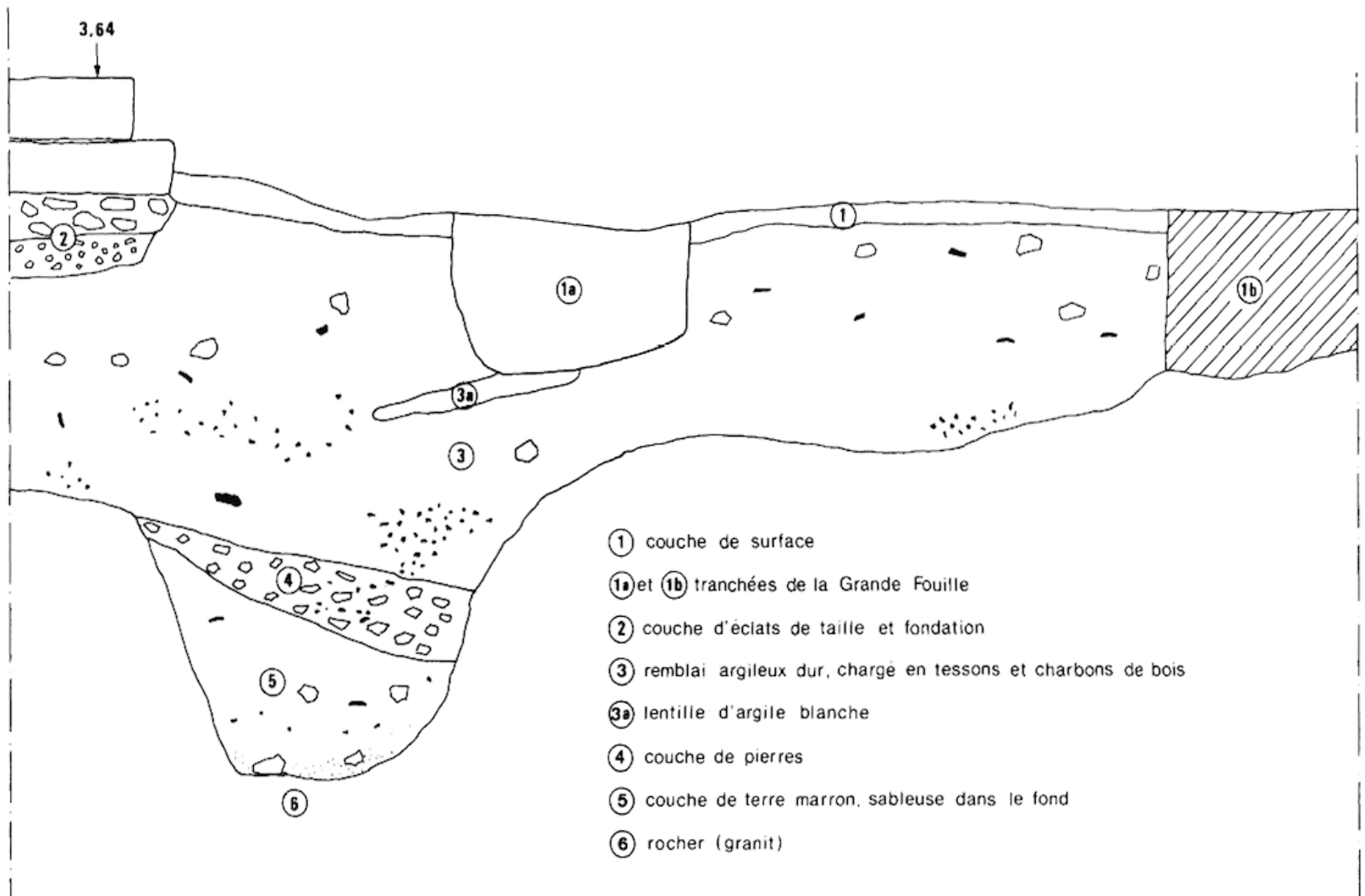

Fig. 2. - Coupe Est dans le sondage 19-190.

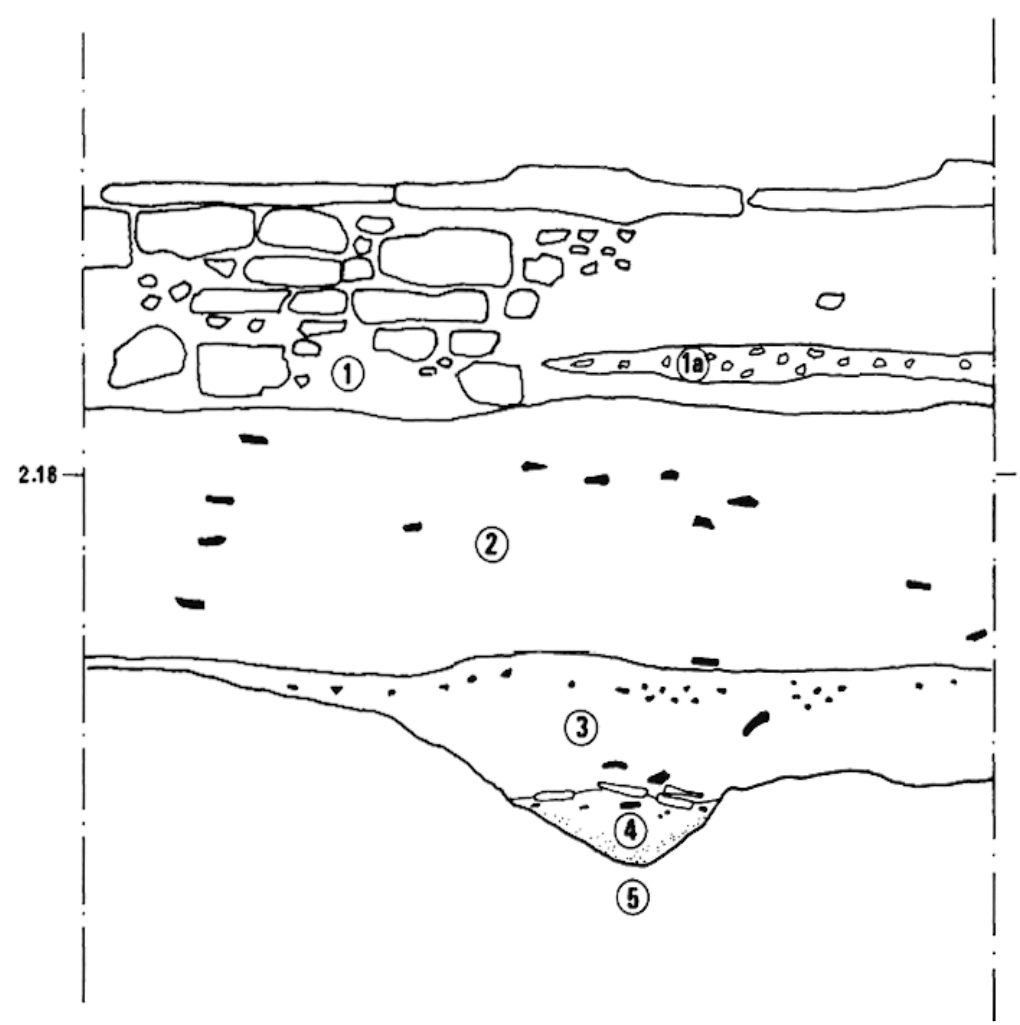

(1) fondation du mur exterieur

(19) couche d'éclats de taille

(2) remblai argileux charge en

tessons et charbons de bois

(3) couche de terre marron

avec tessons et charbons de bois

(4) couche de terre sableuse

(5) rocher (granit)

Fig. 3. - Coupe Sud dans le sondage 11-110.

Fig. 1. - Plan de situation des sondages. 1:200e. 
A l'intérieur du bâtiment nous avons rouvert le sondage 11 de 1975 dans le prodomos, en l'élargissant vers l'Ouest (sondage 110); de mème nous avons ètendu le sondage 10 vers l'Ouest (sondages 100 et 102) et vers l'Est (sondages 101 et 103). Nous avons obtenu la mème séquence stratigraphique qu'en 19-190 (fig. 3). Sous une couche d'éclats de déchets de taille liée à la construction du Prytanée, s'étend la couche argileuse. Elle contient du matériel du Bronze Récent mélé à quelques tessons de corinthien et de vernis noir classique dans sa partie supérieure. On peut signaler un fragment de grand cratère piriforme à décor de losanges et de chevrons datable de l'HRIIIB, de nombreux fragments de coupes à pied haut, de bols profonds monochromes rouge ou brunnoir, des fragments de rhytons et d'un support de vase ajouré. Il faut y ajouter un ensemble abondant de céramique domestique, d'os et de coquillages (dont un triton). Cette couche très dure repose sur une couche de terre plus sombre et plus meuble, de matériel comparable, mais où les os, les coquillages et le charbon de bois sont nettement plus abondants. Cette dernière couche n'est visible que dans les dépressions du rocher, nombreuses dans le secteur, dont le fond est recouvert d'une couche sableuse et humide provenant de la décomposition du granit. Ce sont ces ensembles qui constituent les fosses repérées auparavant.

L'extension vers l'Est du sondage 10 (sondage 101) nous a permis de dégager une vaste cavité circulaire d'un diamètre de près de cinq mètres, taillée dans le granit et munie sur sa paroi Ouest d'une volée de six marches irrégulières suivies d'un court palier puis de deux autres marches qui semblent donner accès au fond (fig. 4). Cet ensemble a été par la suite soigneusement comblé par un blocage de pierres, vraisemblablement au moment de la construction du Prytanée, comblement qui ne fut pas total puisqu'on a, dans ce blocage, appareillé un puits dont l'ouverture se trouvait au niveau du dallage de la cour. Ce puits, profond de $3,80 \mathrm{~m}$ et plus large à la base qu'à l'ouverture (diamètre haut $0,64 \mathrm{~m}$ et diamètre bas $1,22 \mathrm{~m}$ ), a ètè bouché à l'époque romaine (fig. 5). On en a extrait huit fragments d'inscriptions dont quatre actes des archontes et un petit chapiteau de colonne votive portant la dédicace d'un archonte de la fin du ive s. avant J.-C. (fig. 6), des fragments de sculpture d'époque hellénistique (une tête féminine et un petit torse viril) et un ensemble de céramique romaine de la deuxième moitié du $u^{e} \mathrm{~s}$. et du début du ure s. après J.-C. (fig. 7), intéressant pour l'histoire de la Délos impériale. Voisin des groupes $J$ et $M$ de l'Agora d'Athènes, il se compose de pots à eau (M 44), de marmites ( 555 ou J 57) et de grandes cruches à une seule anse (M 125) ainsi que d'amphores 4. Nous avons reconnu dans la cavité circulaire munie d'un escalier une fontaine à degrés permettant d'accéder à la nappe phréatique ${ }^{5}$. La présence du puits d'époque classique nous a empèchés de la dégager entièrement (fig. 8).

La présence de cette structure, fontaine dans un premier temps et puits dans un second, enrichit l'histoire de ce secteur du hiéron. Elle doit certainement être mise en relation avec la couche argileuse repérée dans tous les sondages, particulièrement en 10,110 et 19-190, où le granit présente de profondes dépressions ${ }^{6}$; cette couche semble mettre toute la zone au niveau des points les plus èlevés du rocher (en 10 au bord de la fontaine, le niveau est à $3,26 \mathrm{~m}$ au-dessus de la mer et en 26 , soit à $30 \mathrm{~m}$ plus au Sud, il est à $2,55 \mathrm{~m}$; entre ces deux points, en 110 et 190 , sur le sommet de la couche d'argile il est à $2,93 \mathrm{~m}$ et 2,59 m). Ce remblai comble ainsi les irrégularités du rocher qui devaient constituer autant d'obstacles à l'accès à la fontaine. Il est difficile pour l'instant de dater ce comblement car l'ètablissement du Prytanée a pu faire disparaître les niveaux supérieurs du remblai, comme il a pu le faire aussi pour la superstructure de la fontaine dont on pourrait voir une trace dans les trous creusés dans le rocher visibles en 10 . L'ensemble doit être antérieur au ve s. avant J.-C., date de la construction du Prytanée, et être postérieur à l'époque mycénienne.

La découverte de ce nouveau point d'eau n'est pas sans intérêt pour le problème de l'alimentation en eau du sanctuaire. Les puits sont nombreux dans cette partie de l'île. On en dénombre actuellement neuf encore visibles répartis en arc de cercle d'Est en Ouest dans la partie Nord du hiéron, auxquels il faut ajouter un dixième dégagé en $1903^{7}$ à proximité de l'angle Sud-Est du Temple et un onzième d'époque géométrique fouillé par H. Gallet de Santerre en $1949^{8}$ au Sud-Ouest du Prytanée, tous les deux rebouchés depuis. Neuf sont circulaires avec des diamètres variant de $1,15 \mathrm{~m}$ à $5 \mathrm{~m}$, deux sont rectangulaires, le premier près du péribole Est n'est peut-ètre qu'un bassin ou une citerne', le second dégagé en 1903 est de grande taille puisqu'il fait $5 \mathrm{~m}$ de

(4) H. S. Robinson, Pollery of the Roman Period, The Athenian Agora V, 1959.

(5) Elle n'appartient à aucun des types définis par F. Glaser, Antike Brunnenbauten in Griechenland, 1983. On peut seulement la rapprocher d'une fontaine-citerne de Kastellorizo, W. Wurster, Bauten auf Kastellorizo und Rhos, AM 96 (1981), p. 228 et pl. 75, 1 (je remercie J.-Ch. Moretti pour ce parallèle).

(6) Dans le rapport de l'an dernier, ce remblai est lié à la construction du Prytanée. La découverte cette année de cette fontaine nous amène à reconsidérer ce lien.

(7) $C R A I 1903$, p. 348 et p. 424 , repris dans $B C H 28$ (1904), pp. 268-269.

(8) H. Gallet de Santerre, op. cit., p. 62, note 7.

(9) L. Bizard, Carnet de fouilles 1904, pp. 12 et 13, Archives EFA Del 23. 


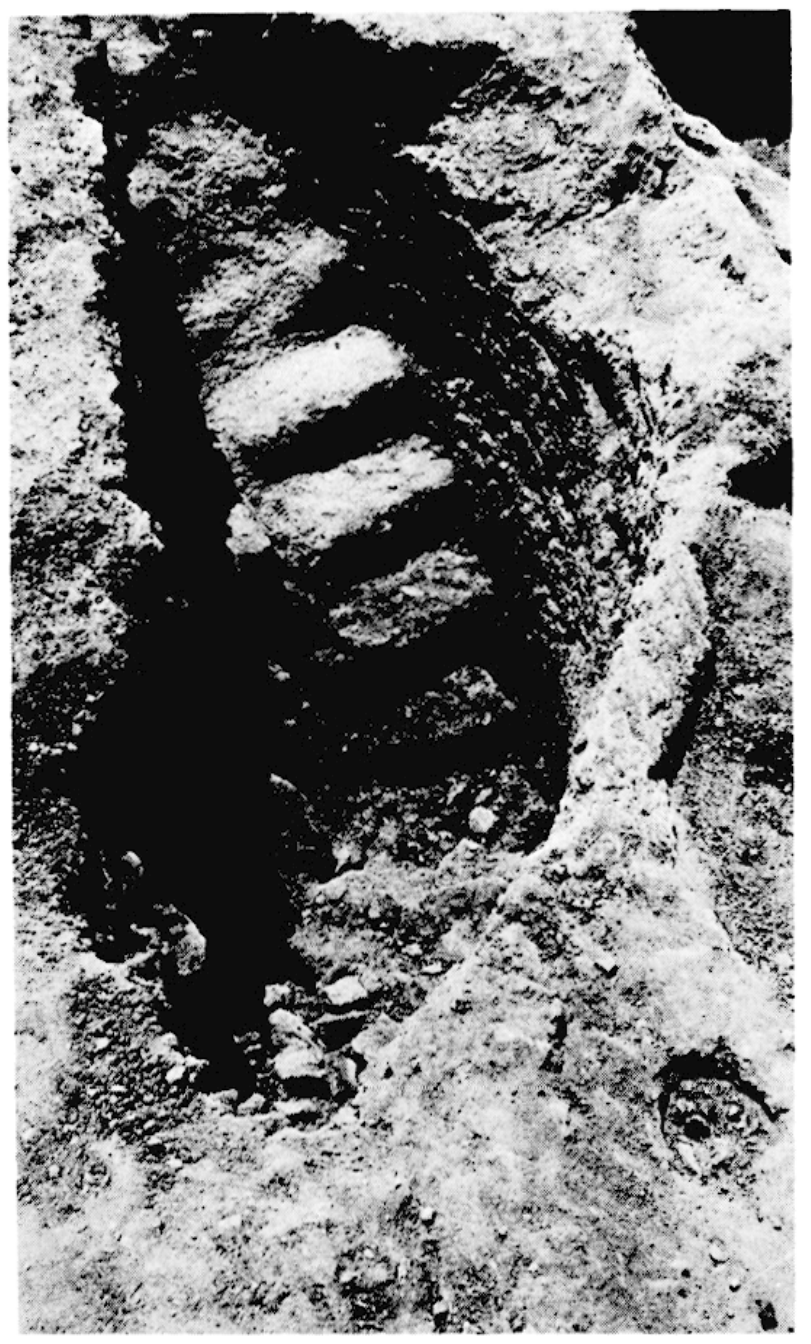

Fig. 4. - Escalier taillé dans le rocher, vu du Nord.

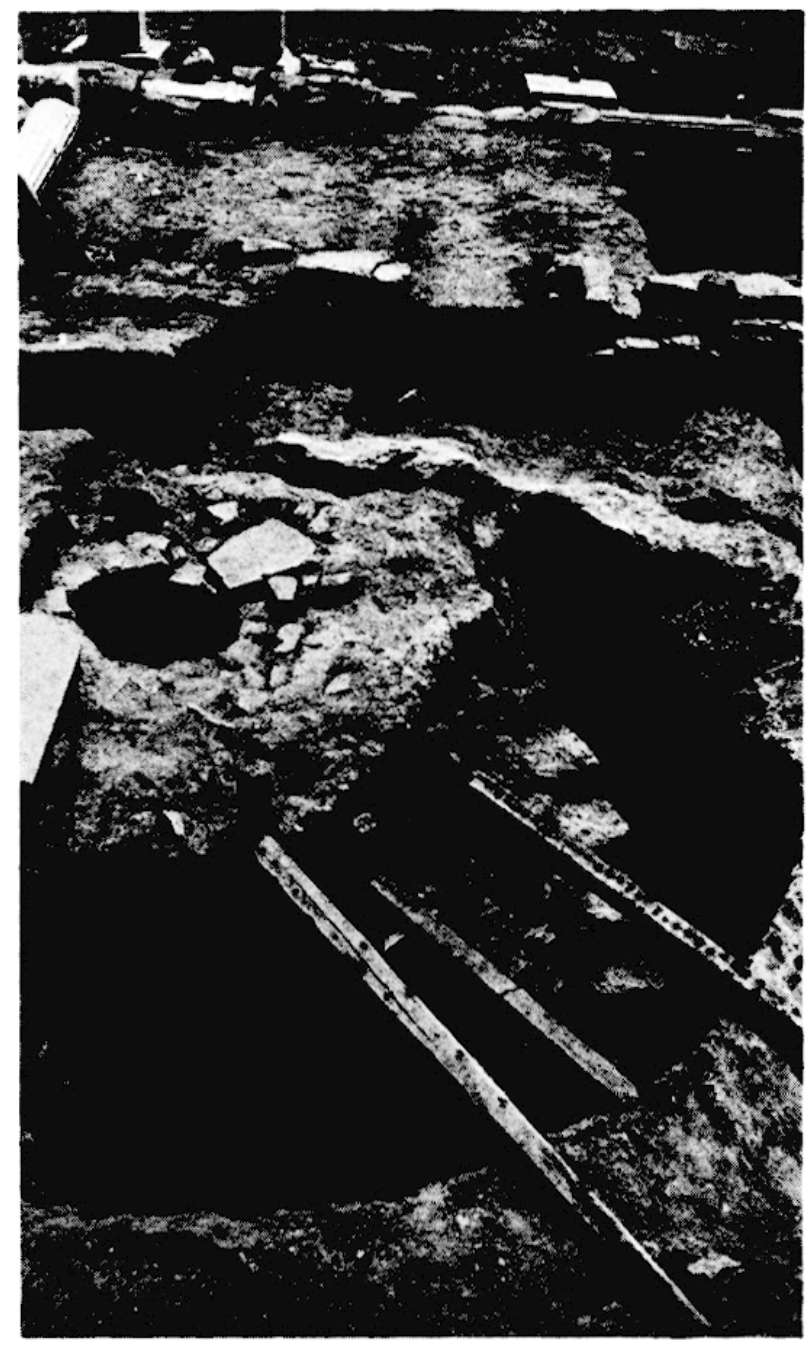

Fig. 5. - Le puits du Prytanée, vu du Nord.

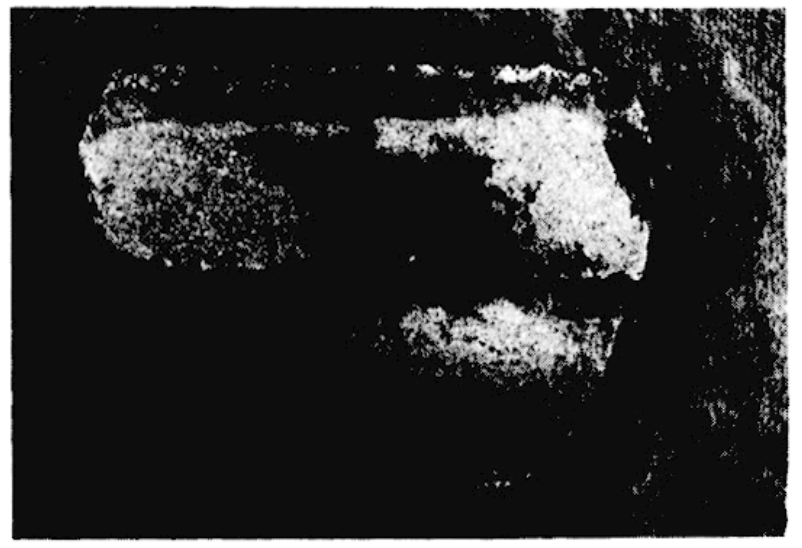

Fig. 6. - Dédicace sur un chapiteau de colonne votive.

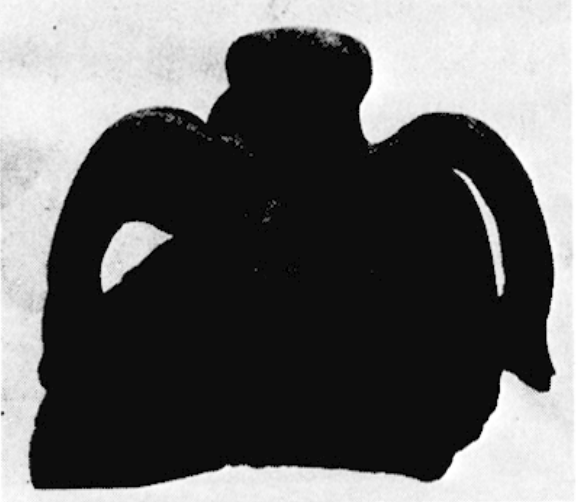

Fig. 7. - Fragment d'amphore. 
Illustration non autorisée à la diffusion

Fig. 8. - Vue d'ensemble du puits et de la fontaine. Dessin Philippe Fraisse. 
côté sur $5 \mathrm{~m}$ de profondeur. Pour autant qu'on puisse en juger encore aujourd'hui - cinq de ces puits ètant en partie comblès - ils sont appareillés dans leur partie supérieure et sont le plus souvent taillés dans le granit dans leur partie inférieure. La seule fontaine connue dans le secteur est la fontaine Minoé, au . Yord du Portique d'Antigone. Elle est munie de marches et taillée dans le rocher, mais elle est de plan carré et ses parois sont

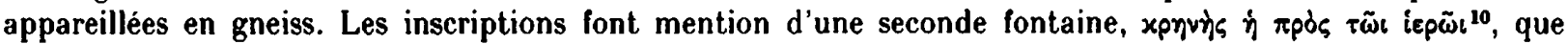
R. Vallois identifie avec le large puits rond à l'angle Nord-Ouest du Trésor $5(G D 16)$; le grand réservoir trouvé en 1903 pourrait être aussi un bon candidat. R. Vallois date la fontaine Minoé de la deuxième moitié du vi* s. avant J.-C. ou du début du ves. La fermeture de la fontaine du Prytanée ou du moins sa transformation en puits intégré dans un bâtiment officiel a peut-être joué un rôle dans l'aménagement de $G D 30$. Les dates de fonctionnement des onze puits ne sont pas connues. Le puits du Prytanée offre pour une fois une chronologie : construit au $\mathrm{v}^{\bullet}$ s. avant J.-C. en mème temps que le bâtiment qui l'incluait, il est comblé en une seule fois à la fin du $\mathrm{II}^{e} \mathrm{~s}$. ou au début du $u^{e} \mathrm{~s}$. après $\mathrm{J}$.-C. avec des matériaux pris sur place (marbres avec inscriptions) et de la céramique commune. Ce type de comblement a déjà été signalé lors de la fouille de certains autres puits ${ }^{\prime \prime}$.

La campagne de cette année aura en outre attiré l'attention sur l'intérêt d'une étude du sous-sol granitique de la plaine du sanctuaire d'Apollon. Si sa nature géologique a déjà fait l'objet d'une première étude ${ }^{12}$, en revanche il n'y a pas eu encore d'enquête systématique sur les aménagements dont il porte les traces. On a, à l'occasion, signalé l'aspect du rocher rencontré au fond de sondages ${ }^{13}$, voire tenté en un endroit de les dècrire en les classant ${ }^{14}$, mais il reste à rassembler ces données dispersées et à les compléter par de nouvelles recherches.

\title{
B. Restitutions architecturales
}

\author{
par Roland Étienne et Philippe Fraisse.
}

Le travail fourni sur les autels de l'Ouest du Prytanée $(G D$ 23) a permis d'assurer la restitution de trois d'entre eux.

\section{a. Autel de Zeus Polieus el d'Athéna Polias, GD $23 E$ (fig. 9).}

L'autel au premier degré devait mesurer 3,30 $\times 2,97 \mathrm{~m}$; le socle comporte deux degrés de $24 \mathrm{~cm}$ de haut; au-dessus, le corps de l'autel mesure 1,52 m de haut. L'autel est identifié par deux inscriptions, $I D, 2607$ et 2608 (sur la localisation de l'autel, cf. R. Étienne, Mélanges G. Roux, à paraitre). Cet autel date sans doute de la fin du vie ou du début du ve siècle.

\section{b. Autel d'Apollon et d'Athéna Paiôn, GD 23 C (fig. 10).}

L'autel est montė sur un seul degré formant la prothysis : $2,73 \times 2,155 \mathrm{~m}$, hauteur du degré, $24 \mathrm{~cm}$. Audessus vient une plinthe de $17 \mathrm{~cm}$ de haut; le corps de l'autel est composé de métopes et de triglyphes (hauteur $66 \mathrm{~cm}$ ) surmontés par un fronton décoré $(50 \mathrm{~cm}$ au faîte). L'inscription ID 47 est gravée sur la face antérieure de la prothysis. L'autel date de la fin du ve s. av. J.-C.

c. Autel anonyme, GD $23 \mathrm{D}$ (fig. 11 et 12).

Seul le premier degré en marbre servant d'assise de réglage est conservé en place $(3,19 \times 3,95 \mathrm{~m})$. On dispose aussi de blocs de couronnement ornés d'un bec de corbin (hauteur $32 \mathrm{~cm}$ ) et d'un fronton lisse, large de $1,65 \mathrm{~cm}$ et haut de $49,5 \mathrm{~cm}$. Deux restitutions sont possibles, l'une avec un degré (fig. 11), l'autre avec deux degrés au-dessus de l'assise de églage (fig. 12). Le type de scellement place cet autel à la fin du ive s. ou au début du uIre s. av. J.-C.

(10) R. VALLoIS, $A H D$ I, p. 193.

(11) $B C H 28$ (1904), pp. 268-269.

(12) L. CAYEuX, EAD IV, pp. 193-196.

(13) A l'Oikos des Naxiens par exemple, P. Courbin, EAD XXXIII, pp. 11-13 et fig. 4 et 5.

(14) CRAI 1910, pp. 311-312 : excavations dans le rocher découvertes au Sud de l'Agora des Déliens. 
Fig. 9. - Autel de Zeus Polieus et d'Athéna Polias. Dessin Philippe Fraisse.
Fig. 10. - Autel d'Apollon et d'Athéna Paiôn. Dessin Philippe Fraisse.
Fig. 11. - Autel anonyme $G D, 23 \mathrm{D}$, solution 1. Dessin Philippe Fraisse.
Fig. 12. - Autel anonyme $G D, 23 \mathrm{D}$, solution 2. Dessin Philippe Fraisse. 


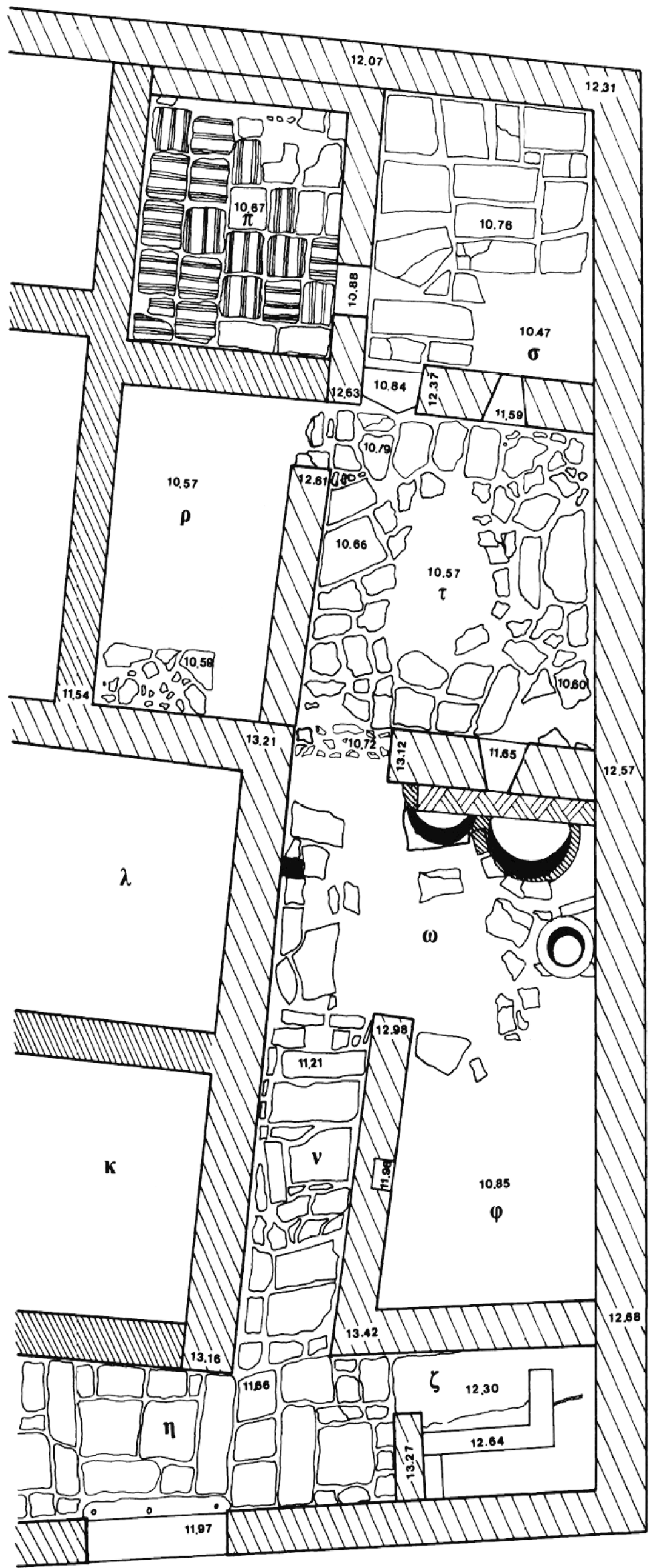

Fig. 13. - La Maison des Sceaux : secteur Est. 


\title{
2. - QUARTIER DE SKARDHANA
}

\section{A. La maison des Sceaux}

\author{
par Gérard SifberT
}

L'objectif des travaux (17 août-11 septembre) était d'achever l'exploration de la Maison des Sceaux dont la partie Est, délimitée depuis 1975 (BCH 100 [1976], p. 800, fig. 13), restait à fouiller. Les travaux dirigés par G. Siebert ont été exécutés avec un effectif de 7 fouilleurs, dont 1 ouvrier ${ }^{15}$. Le Service Archéologique était représenté par M. Panayotis Hatzidakis.

I. Les pièces du Rez-de-chaussée du SEcteur Est de la maison (fig. 13).

1. Entre le vestibule $\eta$ et les latrines $\zeta$ occupant l'angle Sud-Est de l'habitation, le corridor $v$ (fig. 14) dessert une enfilade de pièces dont certaines avaient, au vu des installations et des trouvailles, des fonctions bien

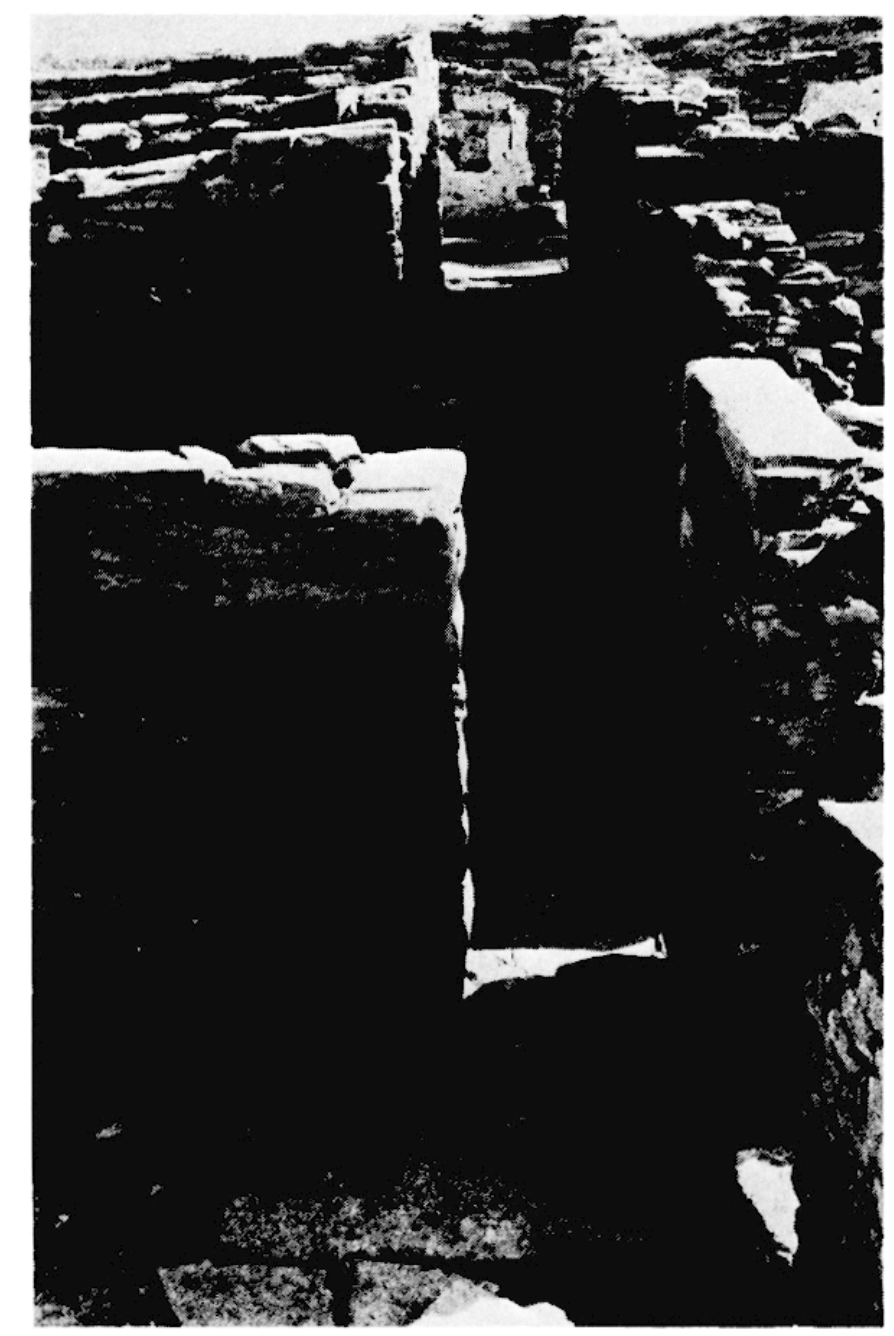

Fig. 14. - Le corridor v, vu du Nord.

(15) Faisaient partie de l'équipe de la fouille, Yolande Siebert et quatre étudiants strasbourgeois, accomplissant un stage dans le cadre de la convention de coopération entre l'École française d'Athènes et l'Université des Sciences Humaines de Strasbourg : Daniel Chevraux, Isabelle Grohens, Frédéric Mercier et Peggy Michaelidou. Les relevés ont été faits par Fr. Mercier. D. Chevraux a assuré la couverture photographique. 


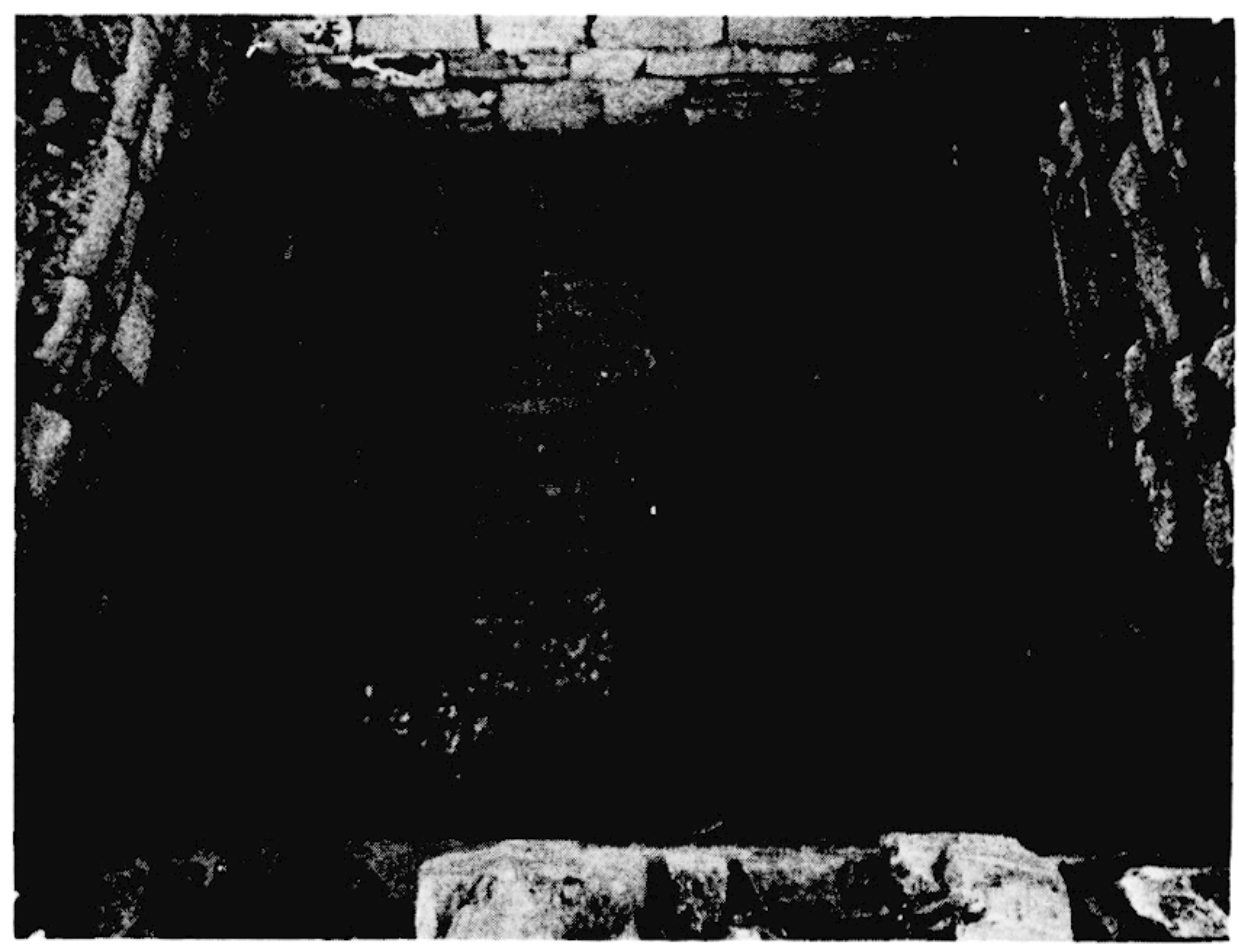

Fig. 15. - La pièce $\pi$.

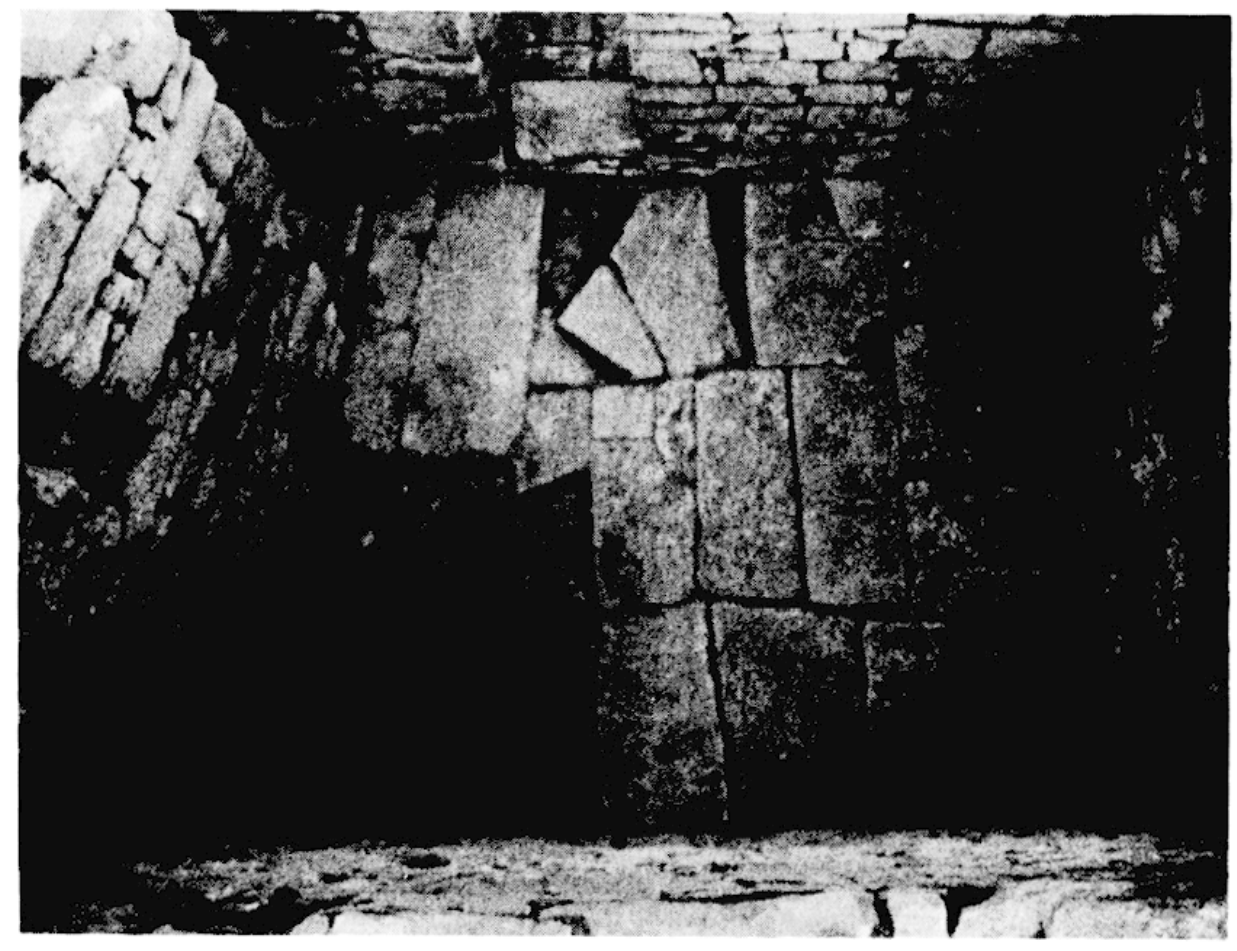

Fig. 16. - La pièce $\sigma$. 
déterminées. Le couloir descend en forte pente vers le Nord, comme il ressort des points hypsométriques notés sur le plan de la fig. 13. Son sol est tapissé de grosses dalles en gneiss qui n'en recouvrent pas tout à fait la largeur; des plaques plus petites comblent les intervalles du côté du mur Ouest et ont été utilisées pour une réfection vers le milieu du passage. Le dallage repose sur un remblai qui s'est tassé et effondré au contact de la zone $\omega$.

2. A l'extrèmité Nord du secteur les sols de deux pièces sont soigneusement aménagés : $\pi$ déjà fouillé en 1975 (BCH 100 [1976], p. 801) comporte un tapis de briques striées (fig. 15), $\sigma$ un dallage de gneiss à l'exception d'un rectangle en terre battue $(140 / 116 \mathrm{~cm})$ dans l'angle Sud-Est. Le sondage pratiqué en cet endroit a révélé l'existence de deux sols antérieurs, dont l'un n'est que la recharge de l'autre, sous un remblai de $20 \mathrm{~cm}$ qui constitue la fondation du dallage (fig. 16). Cette stratigraphie dénote un premier état des lieux qui se laisse aussi constater dans la construction des murs (infra, III, 1). Dans l'angle Nord-Est de $\sigma$, à $1,17 \mathrm{~m}$ au-dessus du sol, une petite niche d'éclairage avait été obtenue par simple extraction d'une pierre d'assise : une lampe "délienne" $s^{\prime} y$ trouvait in situ. Les pièces $\pi$ et $\sigma$ sont relièes par un étroit passage dont le seuil (deux plaques de gneiss superposées, sans implantation de porte) surplombe de $20 \mathrm{~cm}$ le pavement de briques.

Une articulation analogue existe entre $p(B C H 100$ [1976], p. 805) et $\tau$ : étroitesse du passage, absence de fermeture (deux pierres de l'assise inférieure tenant lieu de seuil), dénivellation sensible. Mais c'est en fait tout le groupe $\pi-\rho-\sigma-\tau$ qui paraît former un mème complexe avec $\tau$, la pièce à la meule. Ni la fondation en maçonnerie construite dans l'angle Sud-Ouest de $\rho$, ni le rectangle de terre battue réservé dans le dallage de $\sigma$ n'éclairent beaucoup sur le rôle de ces pièces vides de toute installation fixe, les décombres proches des sols n'ayant livré que du mobilier d'étage. On les considère soit comme des locaux (peut-être des logements) de service, soit plutôt comme des locaux de stockage.

\section{La pièce à la meule.}

Le dallage de la pièce $\tau$ s'incline légèrement d'Ouest en Est autour d'une cuvette centrale en terre battue. C'est l'aire de travail au milieu de laquelle a été découverte une meule en pierre de lave (fig. 18). L'instrument est connu par d'autres exemplaires ${ }^{10}$, mais celui de la Maison des Sceaux permet, pour la première fois, d'en comprendre le fonctionnement à la lumière d'observations précises faites au moment de la fouille. La cuve de la meule n'est pas monolithe mais formée d'une double couronne de blocs ajustés : rectangulaires et incurvés pour la partie basse; trapézoỉdaux pour la partie haute (fig. 19). Des crampons de fer ètaient engagés dans des mortaises creusées en haut et en bas des blocs de la couronne supérieure; les éléments de chaque partie étaient cerclés par un bandeau métallique. D'après une empreinte de rouille d'une largeur régulière de $2 \mathrm{~cm}$ constatée sur la tranche supérieure des blocs trapézoidaux, le cercle de fer qui entourait l'ouverture de la meule était profilé en gouttière. Un cône de la même pierre de lave, probablement muni d'un manchon en bois, a èté recueilli dans les décombres à $1,20 \mathrm{~m}$ au-dessus du sol.

Ce système était trop lourd pour qu'on pût l'actionner à bras d'homme, pierre contre pierre, en versant le grain dans l'interstice. On avait remarqué que la cuve n'ètait pas posée d'aplomb au centre de l'aire de terre battue, mais de guingois et mordant sur le dallage du côté Ouest (fig. 17). Elle ne se trouvait donc pas in silu sur le sol de la petite meunerie ; elle était tombée d'une faible hauteur sans se disjoindre. La preuve archéologique de son installation surélevée a été apportée par la découverte sous un bloc de la couronne inférieure d'un fragment de stuc mural de la même sorte que celui dont étaient truffés les décombres. Autre observation : l'aire centrale de terre battue était jonchée d'un bois carbonisé dont certains fragments, disposés circulairement, adhéraient par du ciment au bord interne du dallage périphérique. Des pierres de calage, de la mẻme espèce que la cuve, dessinaient un cercle grosso modo calqué sur le pourtour de l'aire. Je restitue comme suit le dispositif et le fonctionnement de la meule :

- La cuve de broyage était montée sur un bâti au-dessus d'une cuve en bois maçonnée au sol pour recueillir la farine. L'effondrement et la combustion de cet ensemble expliquent la position de la meule au moment de la découverte ainsi que la nature et la forme des traces observées.

- Le cône de broyage était maintenu au-dessus de la cuve au moyen d'une poulie ancrée dans une poutre du plafond. Il était de la sorte facile d'en régler la hauteur pour l'actionner en déployant l'effort minimal.

- Vers le milieu du mur Est de la pièce, un peu décentrés vers le Sud et espacés de $82 \mathrm{~cm}$, deux trous de boulins $(30 / 30 / 28 \mathrm{~cm})$ sont percés à $75 \mathrm{~cm}$ au-dessus du dallage. Quelque autre installation en rapport avec l'utilisation de la meule était appuyée contre la paroi.

(16) W. Deonna, Le mobilier délien, EAD XVIII (1938), p. 133-135, pl. 392-393. - En dernier lieu : M.-Cl. Amourettr, Le pain el l'huile dans la Grèce antique (Annales Littéraires de l'Université de Besançon 328, Centre de recherche d'Histoire Ancienne 67, 1986), p. 133-147, pl. 20-25. 


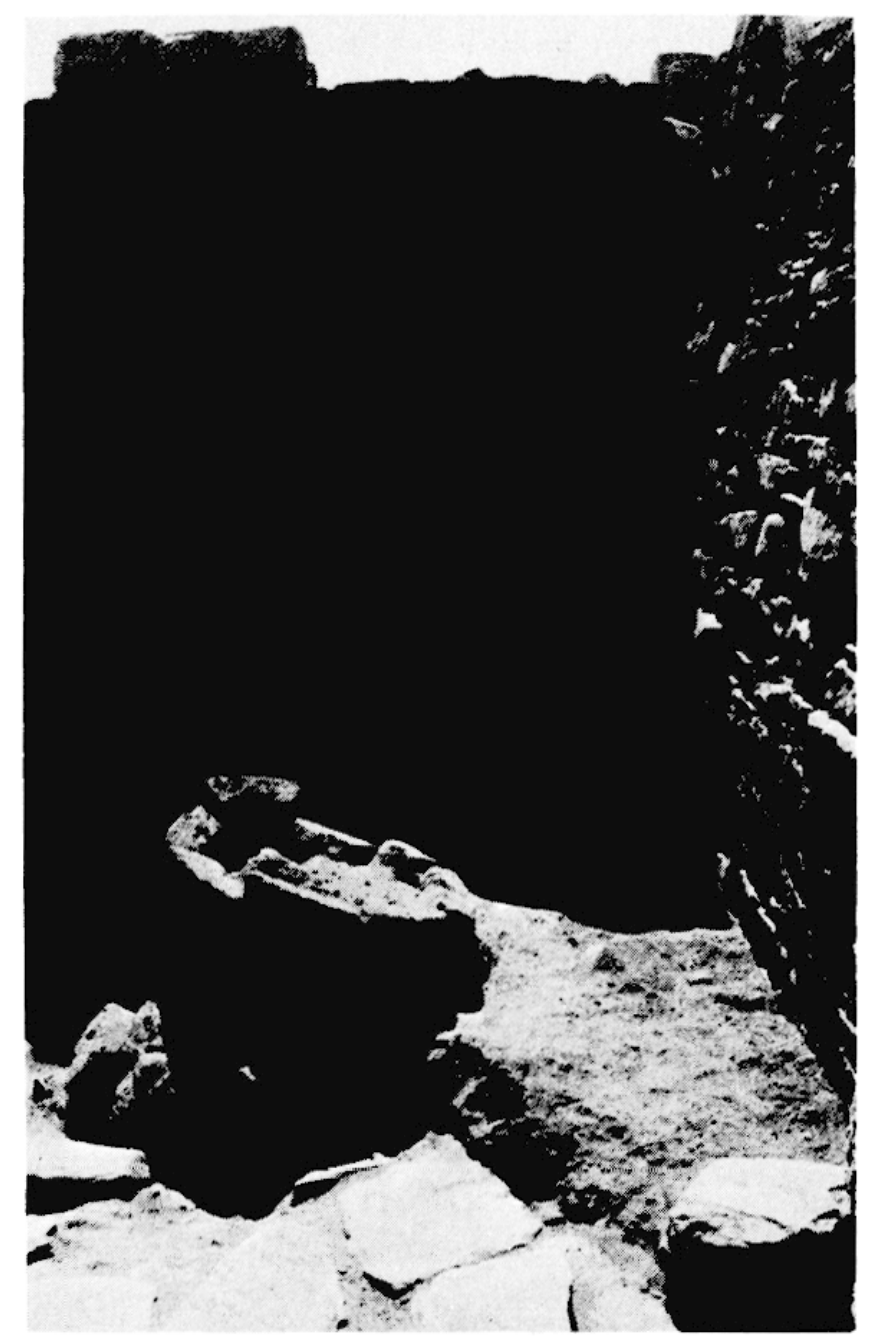

Fig. 17. - Position de la meule en $\tau$. Le muret tardif sur les décombres du passage $\tau / \omega$.

Dans l'angle Sud-Est de $\tau$ une faille irrégulière dans le dallage n'a pas reçu d'explication. Elle existait du temps où l'on travaillait dans la pièce puisqu'une quarantaine de monnaies en bronze ont été trouvées dans la terre qui comblait la cavité ${ }^{17}$. Le sondage pratiqué en profondeur a permis de constater la mise en place d'un remblai tessonneux, alors que dans l'angle Sud-Est de $\rho$ on a rencontré le rocher naturel presque aussitót sous le dernier sol. Tout se présente comme si la partie orientale de la maison avait été construite sur une terrasse obtenue par remblaiement de la pente qui descend vers l'ilât de la Maison des Comédiens.

4. Immédiatement au débouché du corridor se trouvent le cellier de la maison ( $\omega$ ) el son magasin d'amphores $(\varphi)$ réunis en une vaste pièce au sol de terre battue. Deux cuves en argile crue, avec leur soubassement, sont maçonnées contre le mur Nord, la plus grande des deux mesurant $80 \mathrm{~cm}$ de diamètre à l'ouverture (fig. 20). Une brique en forme de parallélépipède servait de marche-pied pour remplir les récipients ou y puiser (fig. 21). Au voisinage de la meule en $\tau$ on peut admettre qu'il s'agissait d'une réserve de grain ${ }^{18}$. Contre le mur Est du cellier $\omega$ était appuyée une grande jarre en terre cuite (diamètre de l'embouchure : $40 \mathrm{~cm}$ ), calée par deux parpaings. Son couvercle en marbre était posé à côté d'elle sur un lit de décombres.

Dans le magasin $\varphi$ treize amphores ètaient alignées le long des murs, les unes couchées sur le sol, les autres non loin du sol au milieu des décombres (fig. 22-23). La position de certaines d'entre elles, allongées

(17) Rien n'indique qu'il s'agit du contenu d'une bourse, comme le petit trésor aggloméré découvert près du seuil de l'oecus major de la maison ( $B C H 100$ [1976], p. 812, fig. 21-22).

(18) Le contenu des cuves n'a pas pu être vérifié au cours de la fouille. Trop fragiles pour être complètement dégagées sans ètre simultanément consolidées, elles ont été remises en terre, dans l'attente de leur restauration in situ. 


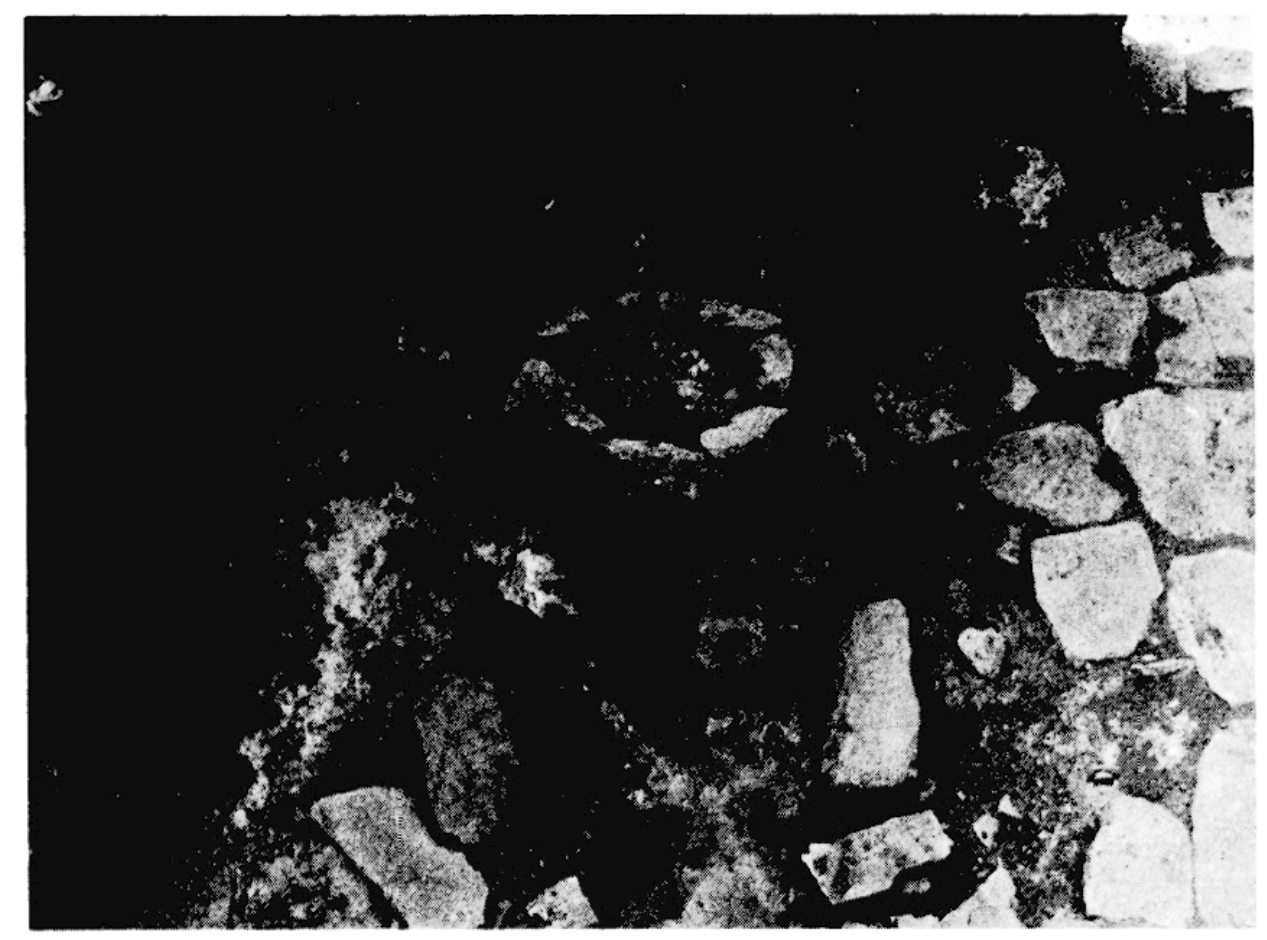

Fig. 18. - La meule en $\tau$, vue latérale.

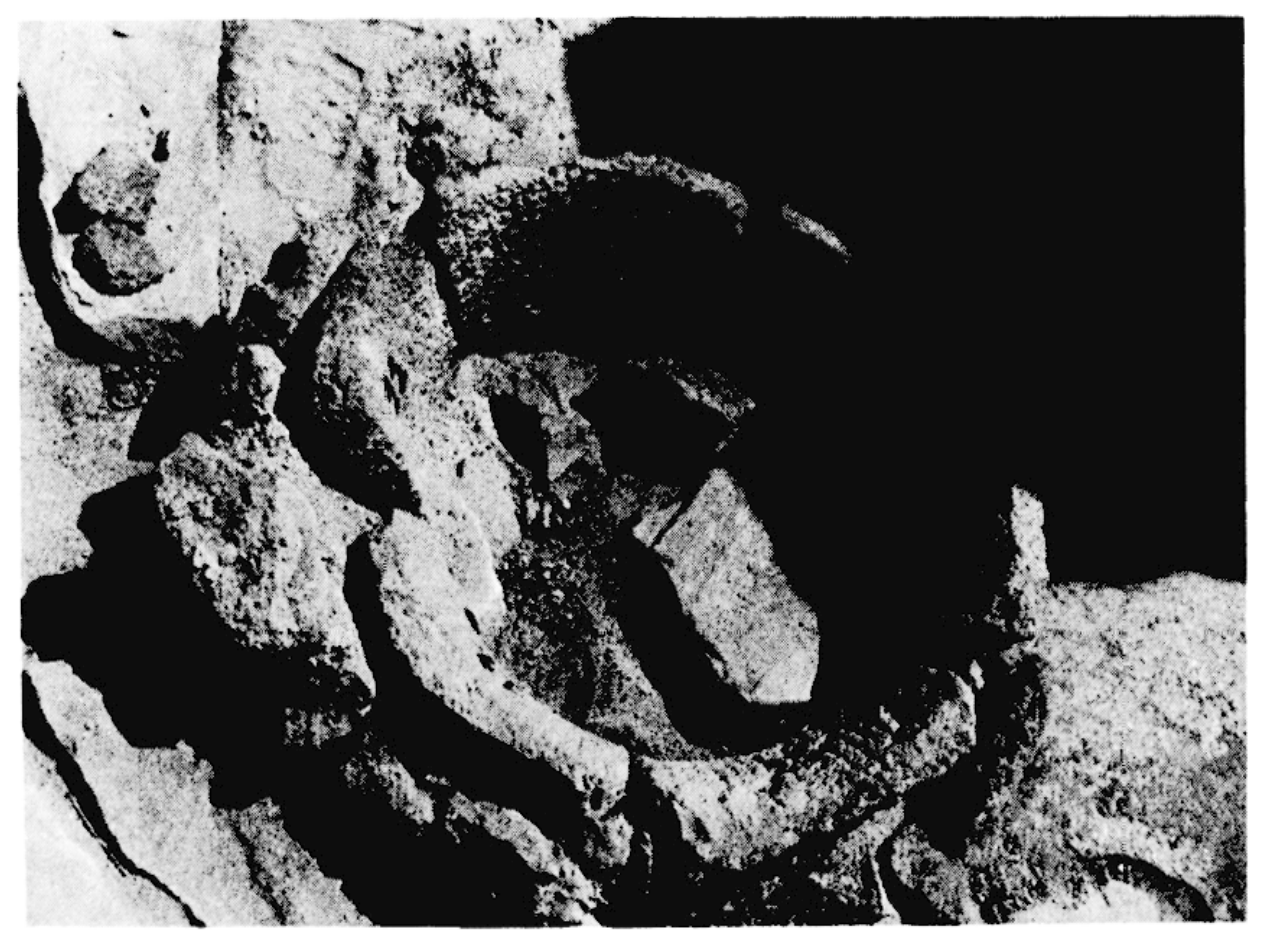

Fig. 19. - La meule en $\tau$, vue du haut. 


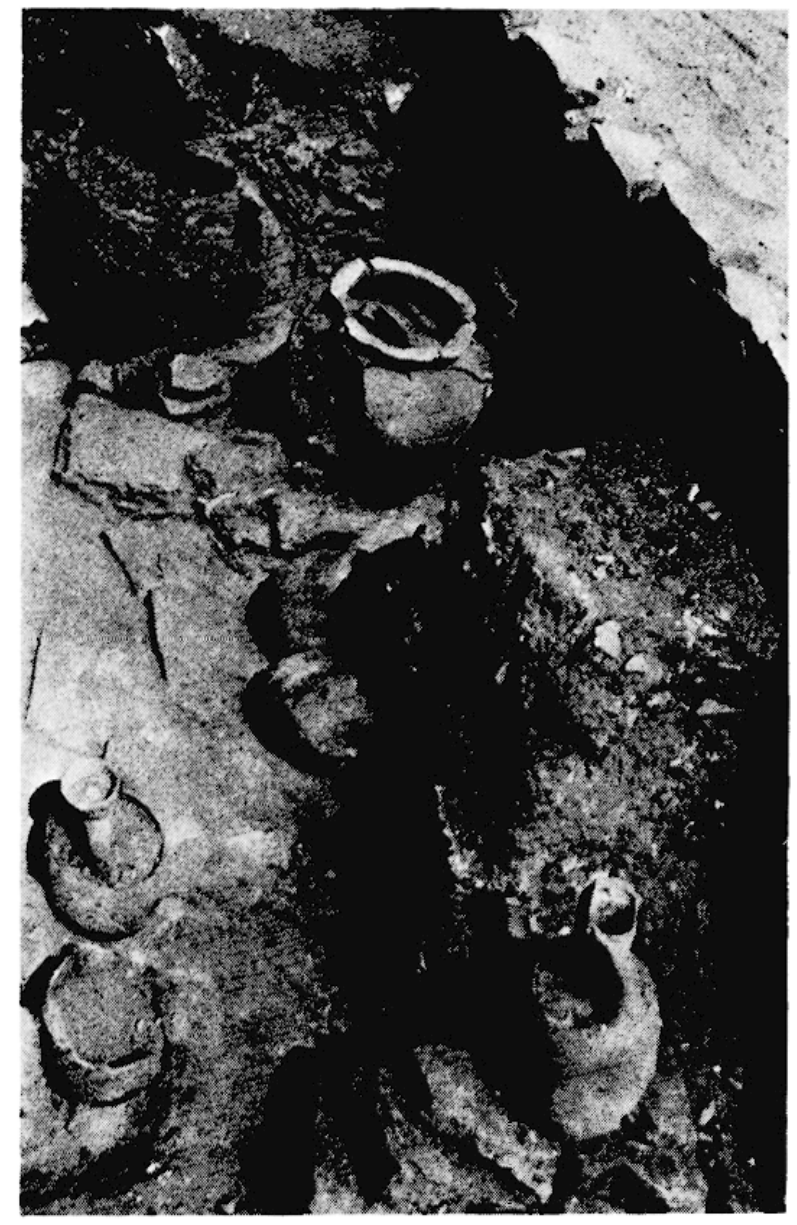

Fig. 20. - Le cellier $\omega$ et le magasin $\varphi$, partie Est.

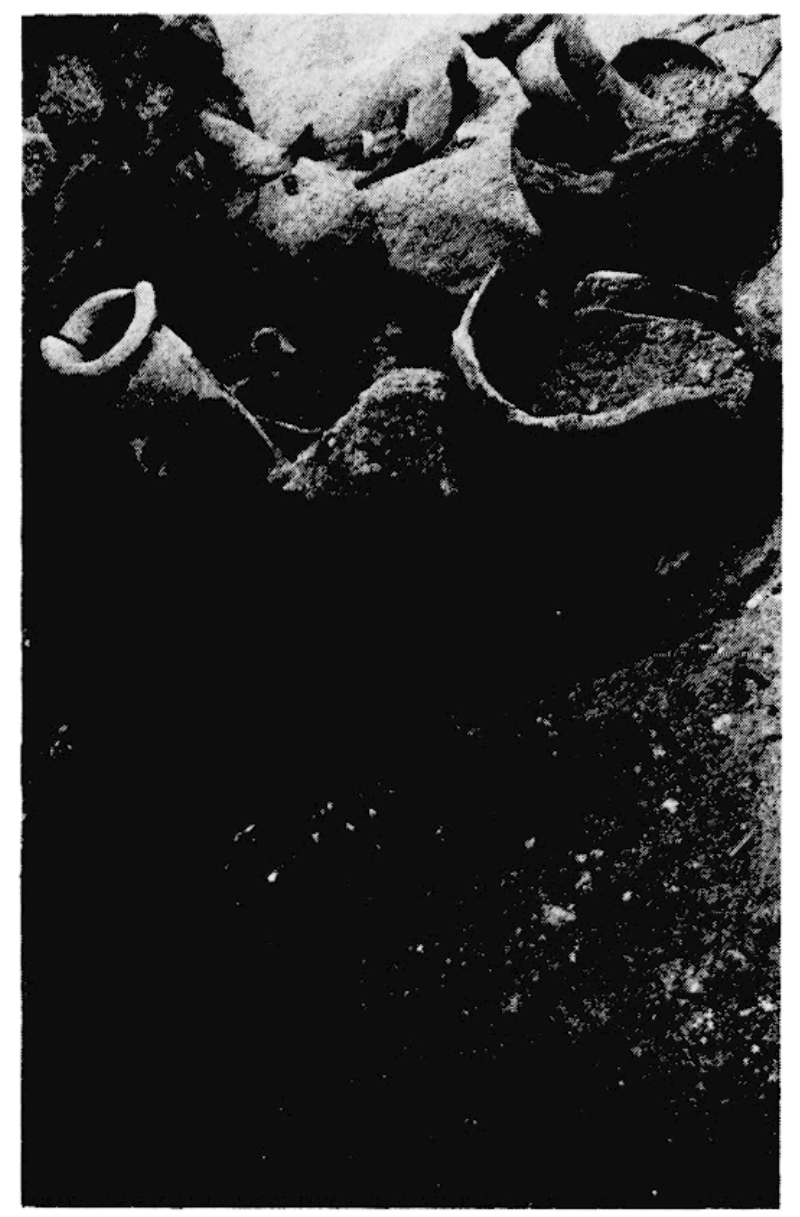

Fig. 22. - Le magasin $\varphi$, partie Nord-Ouest.

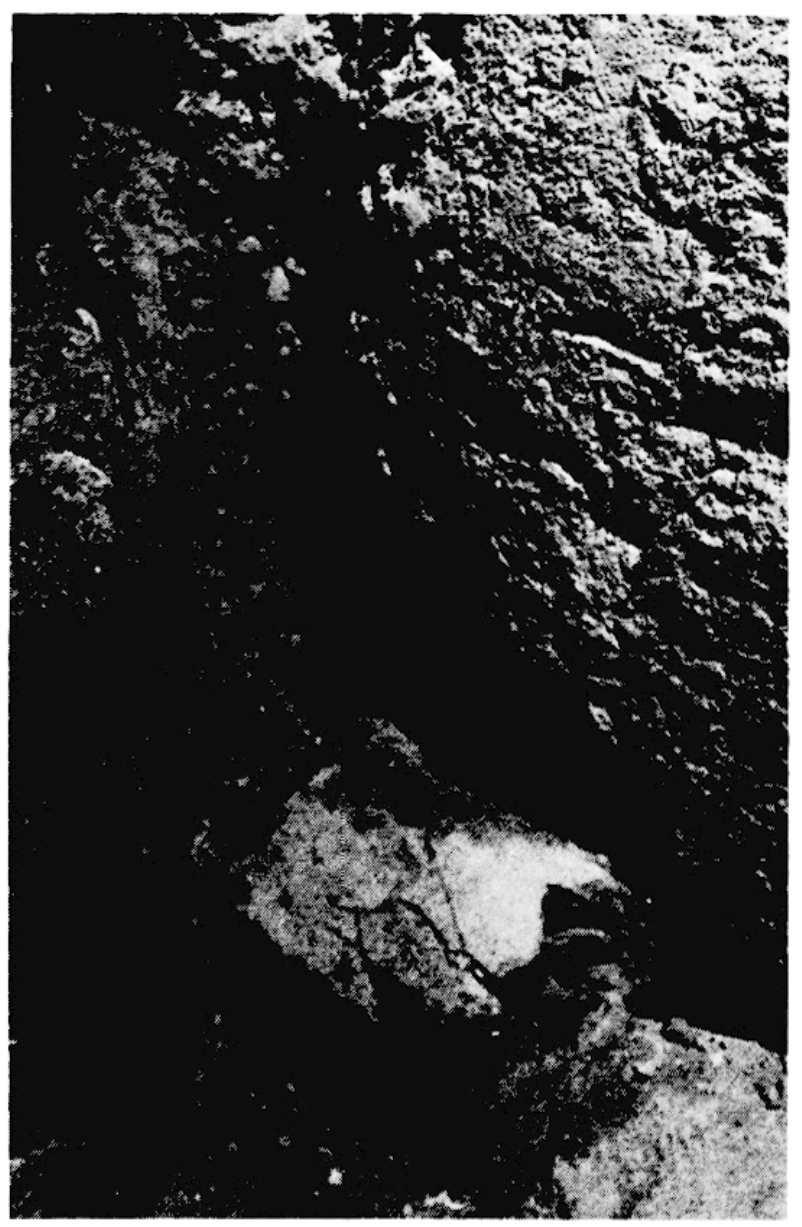

Fig. 21. - Les cuves en terre crue, détail.

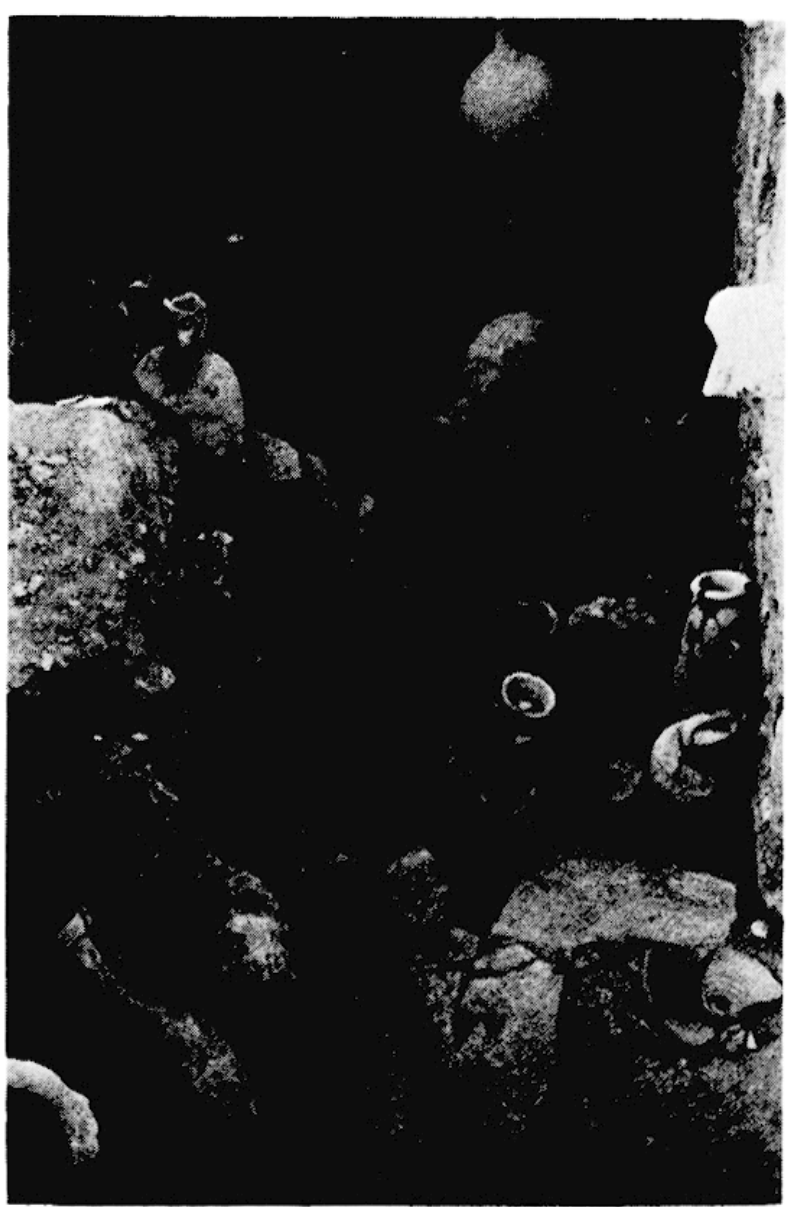

Fig. 23. - Le magasin $\varphi$, vu du Nord. 
horizontalement ou plantées cul par-dessus col, indique qu'elles avaient glissé d'un rayonnage. Une accumulation de bois brûlé, absente en $\omega$, a été constatée le long des murs de $\varphi$. Dans le mur Ouest des saillies de l'appareil de moellons faisaient peut-être office de consoles pour l'appui d'une étagère. Deux niches à lampes servaient à l'éclairage du magasin : l'une dans le mur Sud, partiellement effondrée (mais assurée par les plaques d'enduit réflecteur recueillies en place); l'autre dans le mur Ouest, à une hauteur de 1,25 $\mathrm{m}$, dont des dalles de gneiss et une tuile forment le linteau, les côtés et le plateau. Tandis que nulle part ailleurs dans les pièces de rezde-chaussée du secteur Est on n'a rencontré du stuc en place, les murs du magasin $\varphi$ conservaient des restes d'un enduit brûlé, sans doute blanc à l'origine. Sur la moitié Nord du mur Ouest une plaque plus étendue est incisée de graffites: quelques lettres (un $A$, un $\Delta$, un monogramme $\neg \neg$ ?), mais surtout des faisceaux de traits (21 rayons pour le groupement le plus nombreux). Ces signes, difficiles à décrypter, pouvaient avoir comptabilisé l'état des stocks, selon les entrées et les sorties.

Des amphores ont été découvertes en d'autres endroits de la fouille du secteur Est, en général écrasées dans les décombres (donc provenant de l'étage), plus rarement au sol (deux exemplaires en $\tau$ ). Les fragments exhaustivement recueillis par unités de trouvailles permettront de reconstituer beaucoup d'autres exemplaires et de complèter le faciès amphorique de la maison. La quantité restituable semble largement outrepasser les besoins d'une consommation domestique ${ }^{10}$. Je donne ici la liste des vingt timbres recueillis, dans l'ordre numérique de leur enregistrement au Musée de Délos. La chronologie que j'ai établie pour la destruction de la maison en 69 s'en trouve une nouvelle fois confirmée ${ }^{20}$.

- TD 8087, pièce $\sigma$ décombres de l'ètage, niveau 11,87: Chios; Пap( (fig. 24).

- TD 8088, ibidem : ['I]kowv Proue.

- TD 8089, ibidem : (K)visiov

\section{KYAAK}

ПI

- TD 8090, pièce $\tau$, décombres de l'ètage, niveau 11,80: Cnidien. Tète de taureau. Illisible.

- TD 8091, pièce $\sigma$, décombres de l'étage, niveau 11,70 : 'I\&owv proue (fig. 25).

- TD 8092, corridor v, niveau 1196 ; DORAES (rétrog.) (fig. 26).

- TD 8093, pièce $\sigma$, décombres de l'ètage, niveau 11,70 : 'Aprz(. Lettres incisées (fig. 27).

- TD 8094, pièce $\omega$, décombres de l'étage, niveau 11,60: APEI.

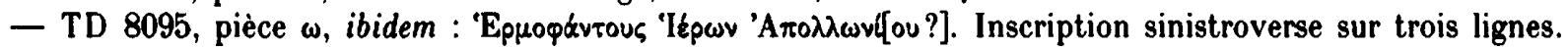

- TD 8096, pièce $\tau$, décombres de l'étage, niveau 11,60: Cnidien. Tête de taureau. Illisible.

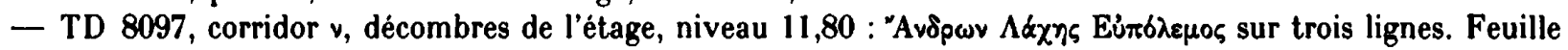
(ig. 28).

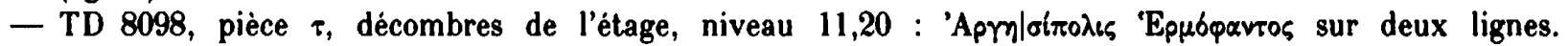
Emblème

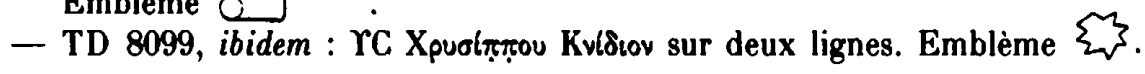

- TD 8100, magasin $\varphi$, sur l'amphore in silu près de la jarre en terre cuite : TRVГ.

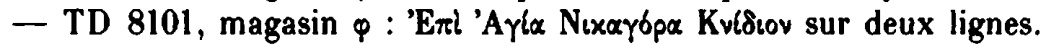

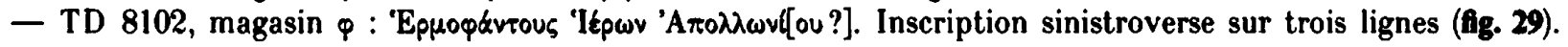

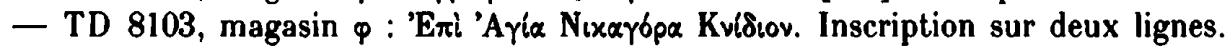

- TD 8104, magasin $\varphi$ : timbre punique circulaire.

- TD 8105, magasin $\varphi$ : ANTIO.

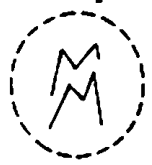

- TD 8118. Timbre ramassé par J.-Y. E. (en p) après la fouille : KA.

(19) Quantité considérable, mẻme abstraction faite de tous les tessons qui ne proviennent pas directement de vases de la maison, mais de l'armature du ciment mural. Cette catégorie de tessons d'amphores, qui présentent des traces de mortier sur une de leurs faces, est aisément identifiable. On en voit en place, par ex. à l'angle extérieur Sud-Ouest de $x$ : plantés à la surface de la paroi de terre séchée, ils accrochent le ciment sur lequel est posé le stuc.

(20) $B C H 111$ (1987), p. 633-636. 


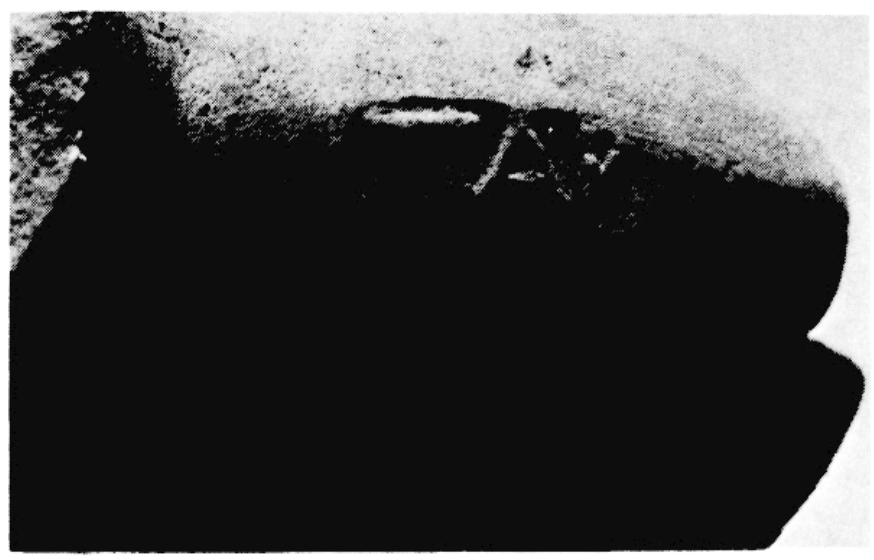

Fig. 24. - TD 8087.

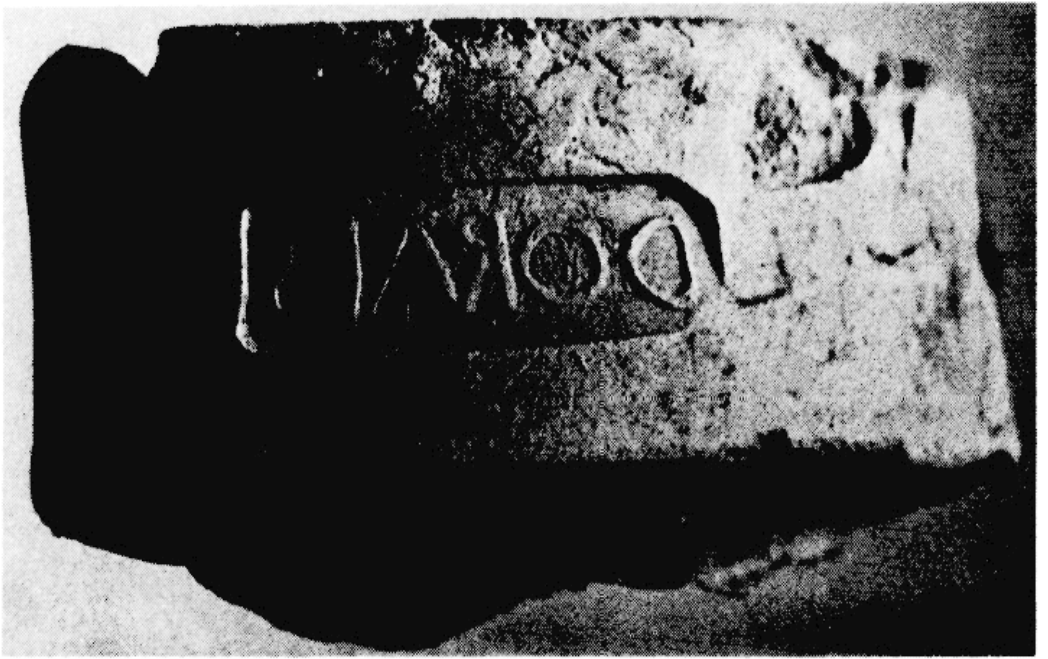

Fig. 26. - TD 8092.

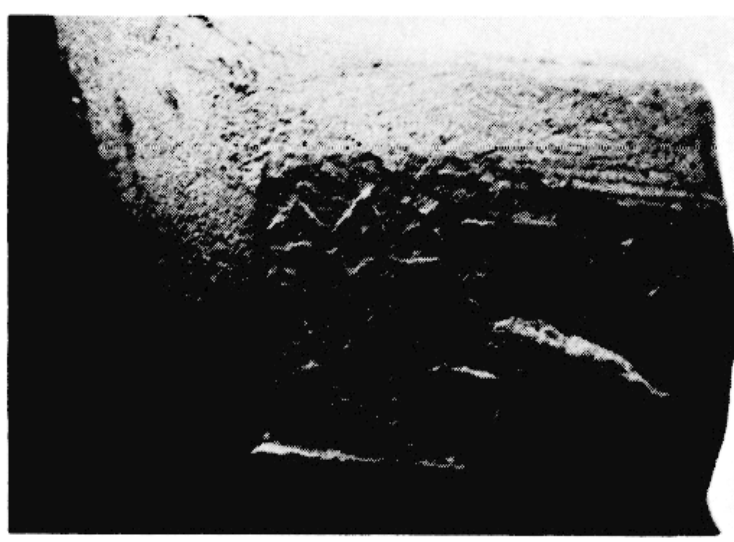

Fig. 28. - TD 8097

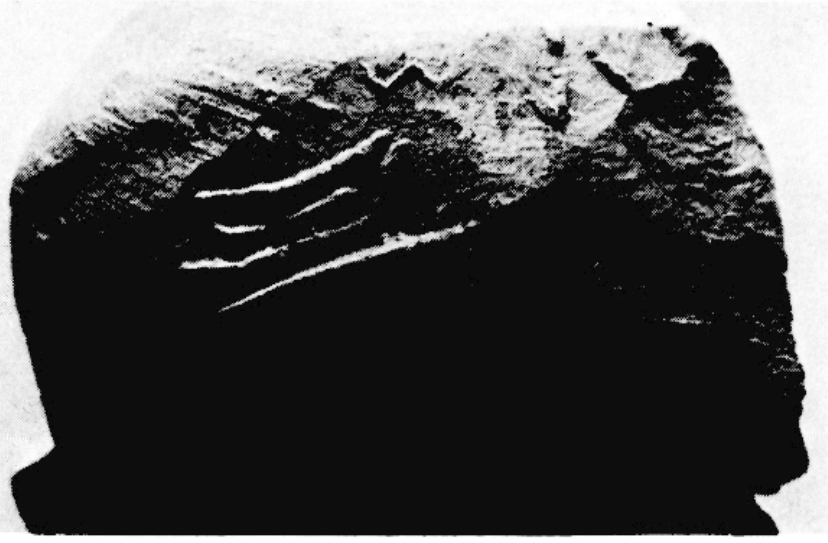

Fig. 25. - TD 8091.

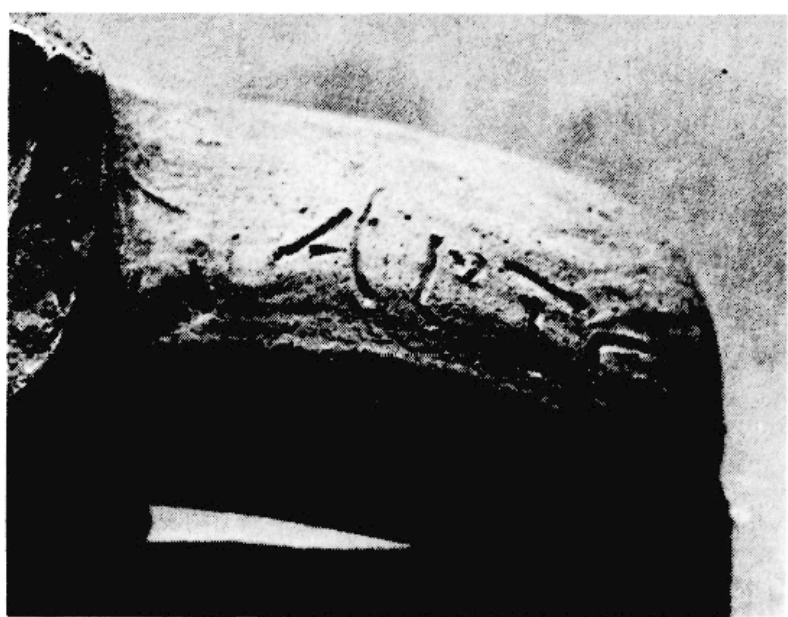

Fig. 27. - TD 8093.

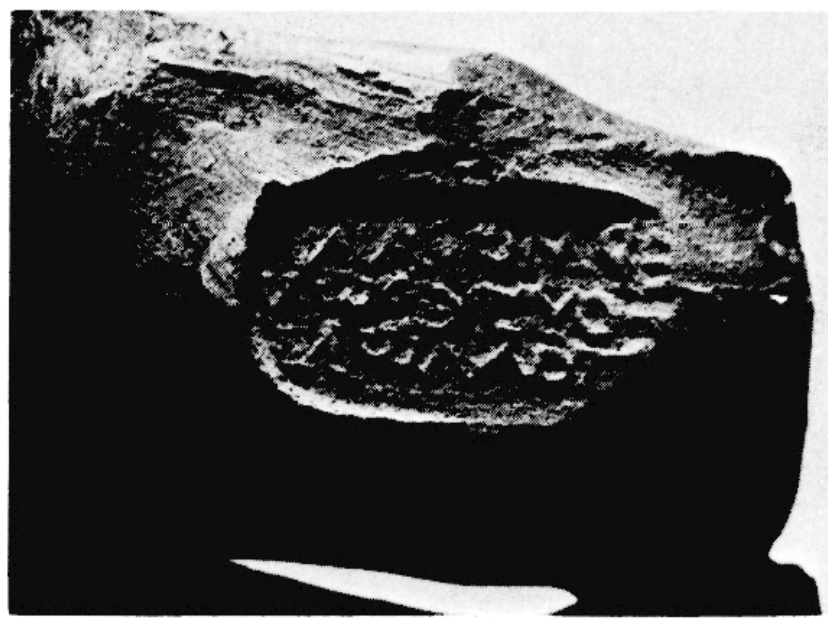

Fig. 29. - TD 8102. 


\section{L'Étage Au-dessus du SEcteur Est.}

Les trouvailles des décombres fournissent un certain nombre d'informations sur l'aménagement et la fonction des pièces d'étage.

1. Comme à l'ordinaire s'y trouvaient les chambres les mieux ornées. Si des centaines de fragments de stuc monochrome rouge ou noir, dispersés partout dans les couches de destruction, ne nous apprennent rien qu'on n'ait observé mille fois, quelques éléments du décor mural se laissent localiser comme suit :

- Au-dessus de $\sigma / \tau$ : bandeaux jaunes encadrés de moulures d'oves et de rais-de-cœurs polychromes; raccordés à des panneaux rouges, des bandeaux jaunes délimités par des tresses à nœuds rouges, des oves blancs, jaunes et verts, des listels noirs.

- Au-dessus de $v / \varphi / \omega$ : osthostates rouges raccordés par des filets à des plages blanches; panneaux bleus et verts avec listels noirs ou rouges; panneaux noirs et jaunes avec moulures d'oves et de rais-de-cœurs polychromes; bandeaux à rais-de-cœurs rouges sur fond clair avec points bleus ; guirlande polychrome sur fond jaune. Plus précisément au-dessus de $\varphi$ : panneaux roses et émeraude; frises de grands méandres rouges et de postes rouges sur fond clair, entre bandeaux bleus et plages vertes.

2. Les mosaiques en opus tesselatum, sans panneaux d'emblema, correspondent aux types les plus communs de Délos. On a pu établir que la région Nord de l'étage comportait des pavements à postes noirs sur fonds jaunes, la région Sud des pavements à postes noirs sur fonds blancs avec cadres noirs et violets. Les tapis de tesselles sont quelquefois raccordés à des mosaïques en éclats de marbre, comme il arrive souvent à la périphérie des pièces. En dehors de l'opus lesselalum quatre variétés de sols se laissent distinguer selon la nature des matériaux ou le soin de l'exécution (fig. 30) : pavements en éclats de marbre lissés de stuc, en éclats de marbre bruts, en tuileaux, en simple mortier (ces deux dernières espèces en plus grande quantité au-dessus de $\tau$ ). Ces sols proviennent les uns de chambres, les autres de galeries et de terrasses. En raison de la masse aussi bien de tuiles que de pavements répartis sur toute l'étendue du secteur Est, je tiens pour assuré que la couverture était faite d'une combinaison de toits et d'auvents d'une part, de terrasses d'autre part.

3. Du mobilier d'élage, ce qui n'était pas de pierre ou d'argile a laissé peu de restes : quelques cabochons d'une porte, deux pieds de coffre en bronze (inv. $87 \mathrm{E}$ 201-202) et une armature en fer (87 E 203) qui appartenait peut-être à une cassa ferrata ${ }^{21}$, une lampe et une coupelle en plomb. Deux plateaux de tables en marbre, l'une avec des fragments du pied, ont été découverts dans les décombres en $\sigma$ (87 E 101) et en $\omega$ (87 E 104-105). De l'étage en $\omega / v$ proviennent aussi les éléments (parfois éclatés en nombreux fragments) d'un grand banc de marbre gris bleuté (87 E 103-d;87 E 108a-b) que j'ai pu entièrement recomposer et préparer pour la restauration ${ }^{22}$. Le plateau $(2 \mathrm{~m} / 41 \mathrm{~cm} / 15 \mathrm{~cm})$, d'un profil galbé, reposait à ses extrémités sur deux pieds schématiquement taillés en pattes de lions et au milieu sur deux piliers qui réduisaient la portée. L'absence de dossier indique que ce meuble était installé contre un mur.

Parmi les objets en argile on signalera la découverte, inédite à Délos, d'une ruche (fig. 31) du type de celles de la ferme attique de Variª ${ }^{23}$. Mais c'est la vaisselle de cuisine et de table qui formait le lot céramique le plus abondant. Son intérêt est de constituer un nouveau dépôt clos bien daté de vases héllénistiques déliens qui s'ajoute à ceux que mes fouilles antérieures dans le quartier de Skardhana ont déjà permis de dèterminer. J'ai évoqué les amphores stockées à l'étage. Le fait que la cuisine et le lieu ordinaire des repas s'y laissent également situer vaut d'ètre souligné. On imagine sans peine l'usage que les familles pouvaient faire des terrasses les plus retirées de leur habitation, comme c'était le cas ici dans le secteur Est de la Maison des Sceaux.

4. L'étage comportait une chapelle domestique, comme il ressort de la découverte d'un relief votif et d'un thymiatérion inscrit.

a) Brûle-parfum en marbre blanc, Musée de Délos inv. A 7725. Provenance : corridor v, à $15 \mathrm{~cm}$ au-dessus du sol, à proximité d'un autre brûle-parfum en terre cuite décoré dans la technique des lagynoi polychromes (ifg. 32). Hauteur de l'objet : $15 \mathrm{~cm}$; diamètre à la base : $8 \mathrm{~cm}$; profondeur de la coupelle : $5 \mathrm{~cm}$. Moulurations à

(21) $B C H$ Suppl. I (1973), p. 566-571.

(22) Les éléments de ce banc sont entreposés dans la cour $\theta$ de la maison.

(23) J. E. Jones - A. J. Graham - L. H. Sacket, "An attic Country House below the Cave of Pan at Vari", $A B S A 68$ (1973), p. 443-452, fig. 18-21 (dessins) et pl. 75-76, 78-80, 83-85. La ruche délienne était entreposée à l'étage au-dessus de $\sigma$. 


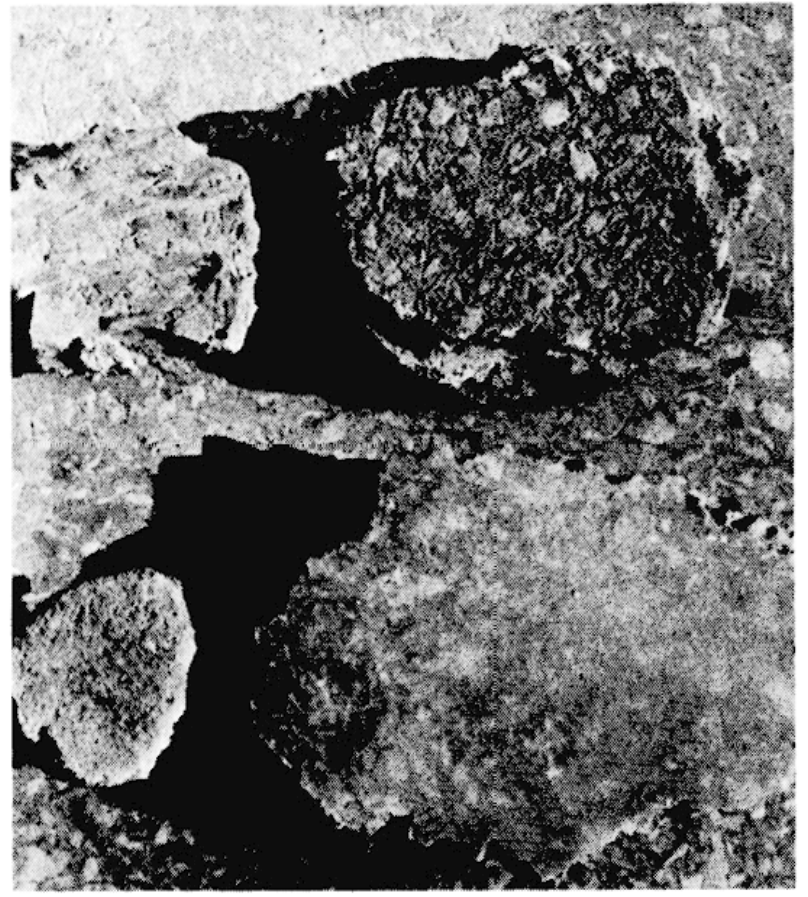

Fig. 30. - Types de pavement d'étage.

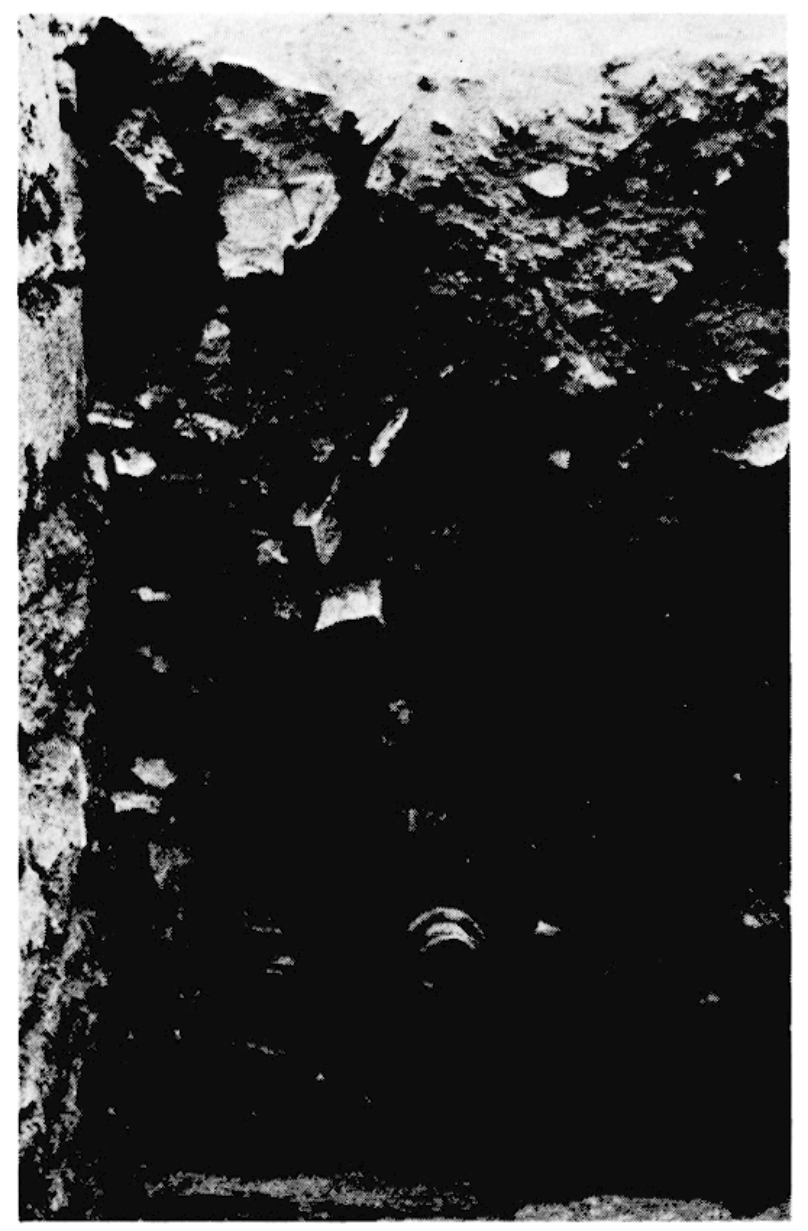

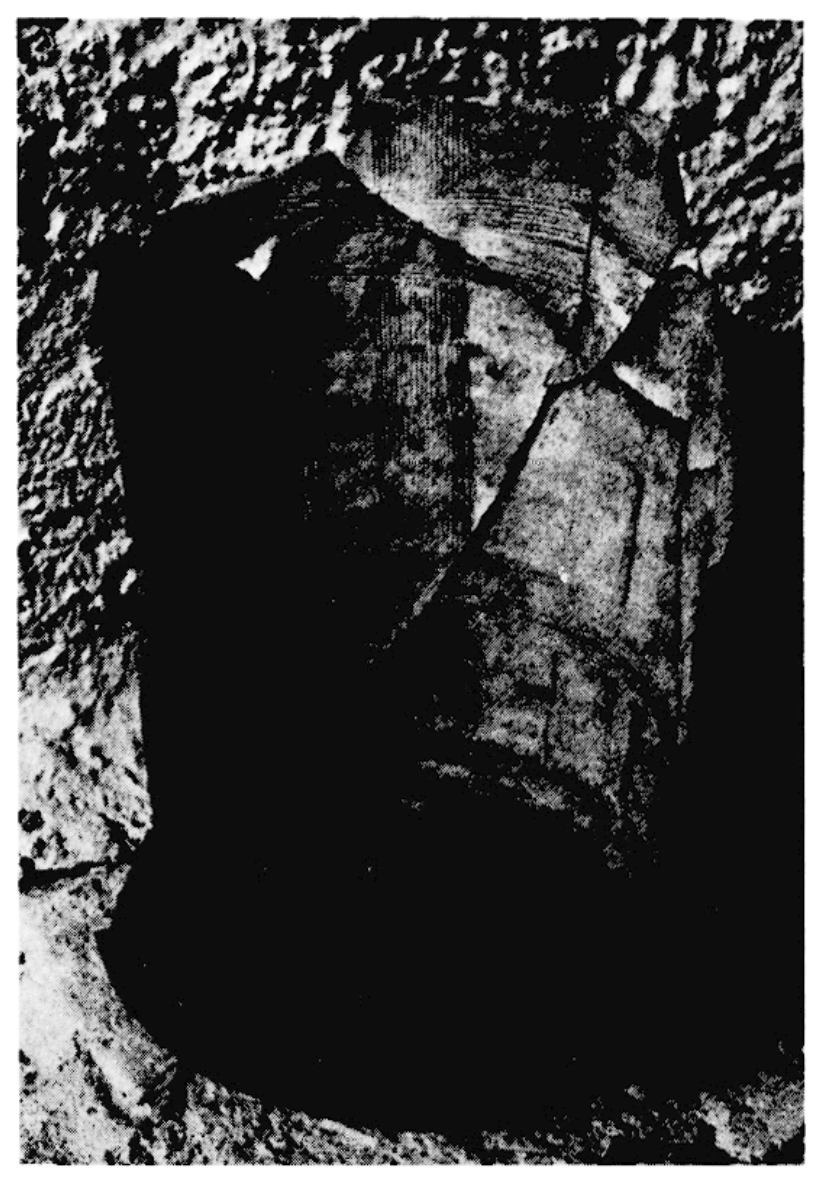

Fig. 31. - Ruche, avant restauration. Musée de Délos, 87 E 1.

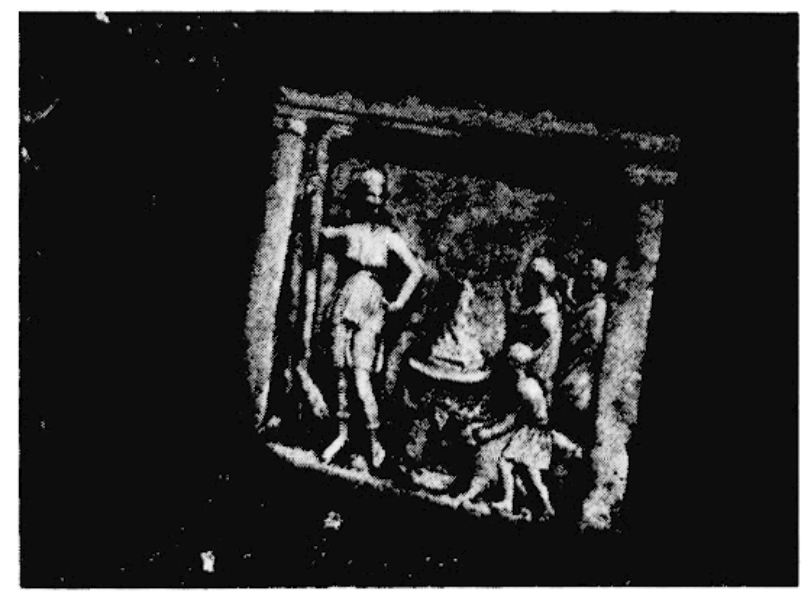

Fig. 33. - Relief votif in silu en $\omega$.

Fig. 32. - Brûle-parfum in situ en v. 
la coupelle et à la base qui comporte un anneau poli de $1,5 \mathrm{~cm}$ de large entourant un disque de $5 \mathrm{~cm}$ travaillé à la gradine. Toutes les faces sont brulées par l'incendie; la coupelle est noircie par la combustion des aromates ${ }^{\mathbf{4}}$.

Le texte est aligné à gauche, mais selon une légère oblique de haut en bas (fig. 34-35). La gravure sur six lignes est hésitante. Légers apices à certaines lettres $(\mathrm{A}, \Lambda, \mathrm{H}) ; \Sigma$ à barre brisée, $\mathrm{A}$ à barre droite. Aucun problème de lecture :

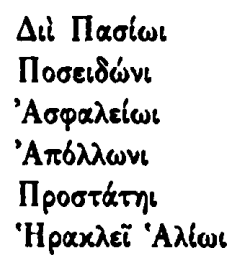

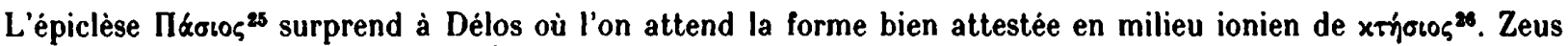
Pasios se rencontre en Arcadie, à Égine, à $\operatorname{Cos}^{27}$. 'A $\propto \varphi \alpha \lambda \lambda_{\varepsilon} \circ \varsigma$ se trouve, dans les dédicaces déliennes à Poséidon,

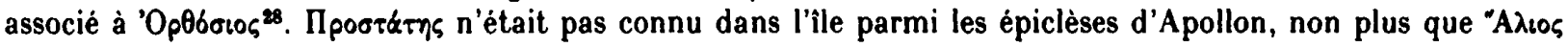
parmi celles d'Héraclès ${ }^{20}$. L'Héraclès de la mer semble être une version du Melkarth de Tyr, celui qui protégeait contre les dangers de la navigation. Les Italiens de Délos (en particulier les marchands) honoraient pour les

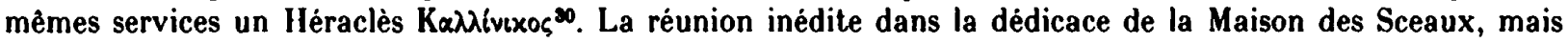
nullement insolite, des quatre divinités et héros, avec le groupement des quatre épiclèses, exprime les aspirations à la sécurité pour les biens domestiques : rei familiaris tutoris dit à propos de Zeus Pasios le commentateur de la Sylloge. L'invocation de l'Héraclès de la mer trahit des voux plus spécifiques, qui ne sont pas inattendus dans la maison de l'homme d'affaires qui conservait chez lui des archives de contrats et qui, à en juger par la quantité d'amphores qu'il stockait dans ses réserves et, occasionnellement, jusque dans son oecus major, avait sa part au commerce maritime de Dèlos ${ }^{31}$.

b) Relief en marbre avec la représentation d'un sacrifice à Arlémis. Musée de Délos A 7724. Provenance : près de l'angle Nord-Est de $\omega$, au niveau 11.65 en appui contre le mur Est, sur un coussin de cendres, la face sculptée tournée vers l'Ouest (fig. 33). Dimensions : $41,5 / 37,5 / 10,5 \mathrm{~cm}$. La plaque, simplement équarrie à l'arrière et sur les tranches, était encastrée dans un mur d'étage qui l'a entrainée dans sa chute.

Le relief est de facture artisanale (fig. 36), certaines formes (le chien, le porc, la guirlande à bucranes sur l'autel) ètant outrancièrement simplifièes. Les deux orants, engoncés dans leurs manteaux qui couvrent les bras presque jusqu'aux poignets, ne font l'objet d'aucun effort de différenciation de la part du sculpteur. En revanche, le petit victimaire, qui pousse la bête par l'encolure, est d'un mouvement de trois-quarts/profil plein de justesse. Artémis, elle, est tributaire d'un bon modèle post-praxitélien. Campée avec coquetterie, une main à la hanche, l'autre appuyée sur une immense torche comme sur un sceptre, elle porte la tunique courte et les bottes de la chasseresse. La souplesse et les effets de transparence dans la draperie mettent en valeur l'élégance de la pose devant les adorateurs de la pompè. La scène se déroule dans un sanctuaire indiqué par les piliers et par un entablement que couvre un toit sommairement esquissé. Dadophore, court-vètue, flanquée du chien, elle combine des attributs qui sont aussi bien ceux de la déesse de la chasse que ceux de l'Hécate infernale ${ }^{*}$. Le sacrifice du porc au lieu de celui plus habituel de la chèvre distingue le relief de la Maison des Sceaux de ceux du sanctuaire d'Artémis Lochia sur le versant oriental du Cynthe ${ }^{33}$ (fig. 37).

(24) Le nouvel exemplaire entre dans la typologie dressée par W. DeonNA, EAD XVIII, p. 371-385, pl. CIII-GIV.

(25) Le $\Pi$ initial est certain : il ne s'agit pas du Zeus Køolos du Sérapieion A (Ph. Bruneau, CDH, p. 241).

(26) A Délos mème, pour une dédicace aux dieux égyptiens et à Zeus : ID 2186 (CDH, p. 242).

(27) H. Schwabel, RE X A (1972), s.v. «Zeus", col. 350. - Pour Tégée : ArchEph. 1911, p. 152 no 7 ; M. Jost, Sanctuaires el culles d'Arcadie (ÉtPélop IX, 1985), p. 276-277; cf. etiam Fr. SAlvı̃, BCH 82 (1958), p. 219-221. - Pour Cos : Sylloge, p. 265 (inscription de Diomédon). - Inversement Krijolos se rencontre en milieu dorien, à Théra : IG XII $3, n^{\circ} 1361$.

(28) $C D H$, p. 265.

(29) Ibidem, p. 170 et $405-411$.

(30) Ibidem, p. 408-412. L'Héraclès phénicien de Délos avait rang divin.

(31) $B C H 100$ (1976), p. $810-811$.

(32) G. Sifbert, Artémis Sóteira à Délos", BCH 90 (1966), p. 447-459.

(33) A. Plassart, Les sanctuaires et les culles du Mont Cynthe, EAD XI (1928), p. 298-312, fig. 247-256; Ph. Bruneau, CHD, p. 191-195. 

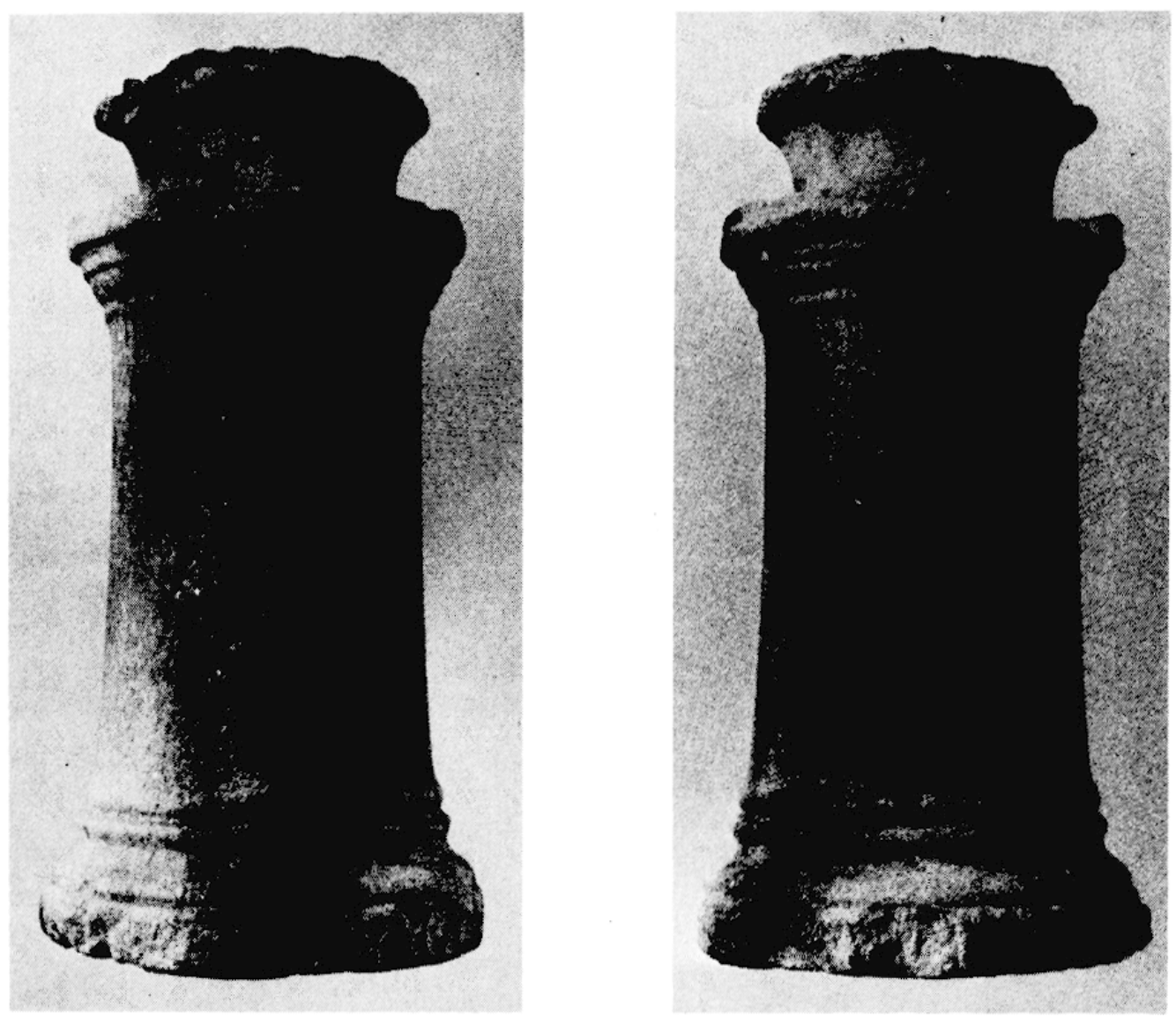

Fig. 34-35. - Brûle-parfum inscrit. Musée de Délos A 7725.
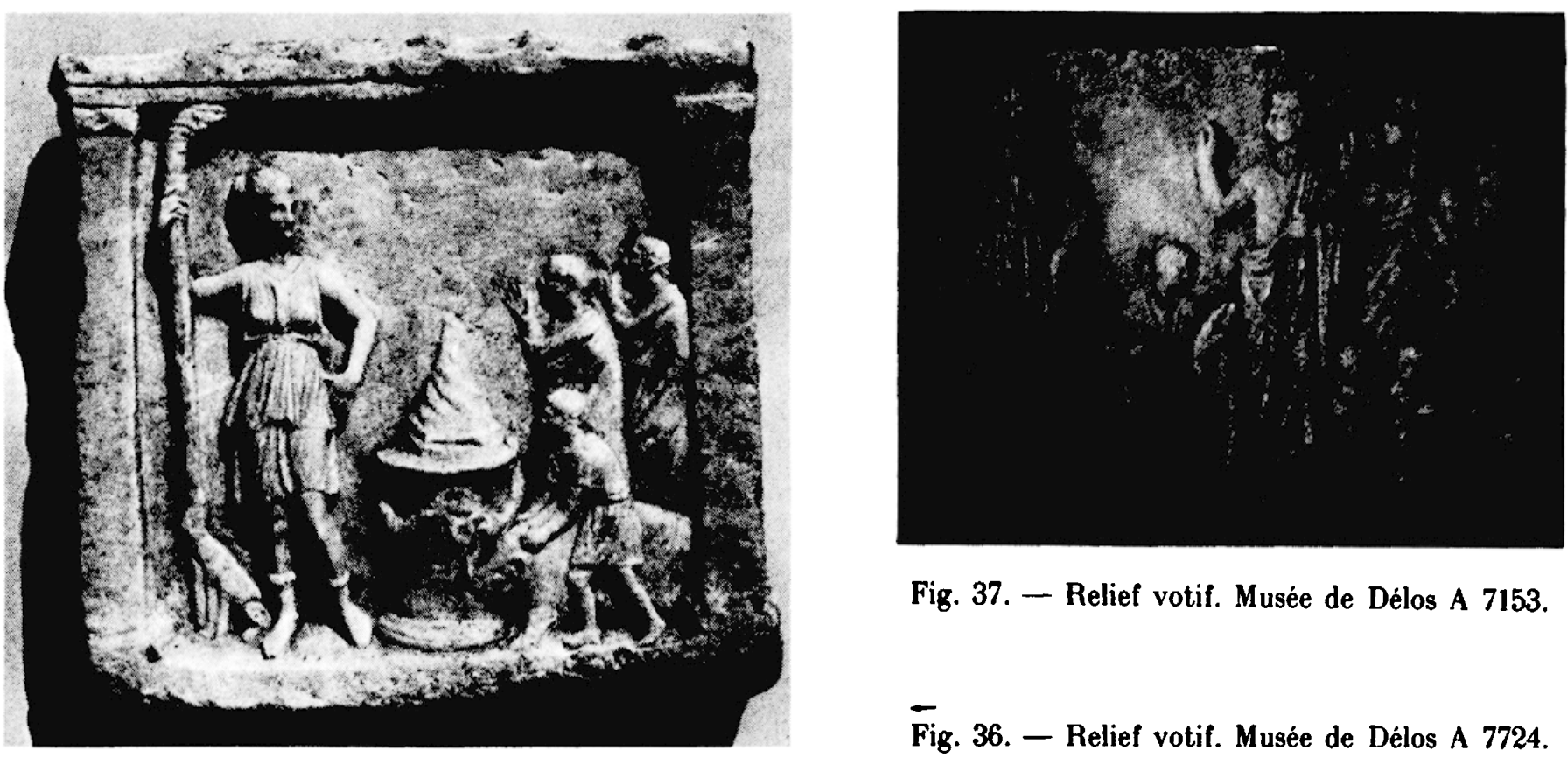

Fig. 37. - Relief votif. Musée de Délos A 7153.

Fig. 36. - Relief votif. Musée de Délos A 7724. 


\section{Transformations.}

1. La distribution de l'espace du secleur Est dans la dernière phase de l'occupation ne remonte pas à la construction primitive. De mème qu'à l'Ouest son contour avait été modifiée du côté de la Maison de l'Épée (BCH, 111 [1987], p. 640-641), de mème la Maison des Sceaux avait connu des réaménagements importants sur sa bordure orientale. Le plan de la fig. 13 fait apparaitre aussitòt que deux grandes pièces d'un premier ètat ont été transformées par cloisonnement en quatre pièces plus petites. La symétrie du nouveau complexe, l'alignement des ouvertures dans l'axe du couloir $v$, les différences entre les murs anciens bien bătis et les cloisons d'appareil hétéroclite qui y prennent appui, sans le moindre liaisonnement, sont autant de preuves d'une opération conçue et exécutée systématiquement ${ }^{34}$. Le doublage inutile du mur. Nord de $\pi$ s'explique peutêtre par la nécessité de couvertures dénivelées.

D'autres indices sont fournis par les canons de lumière aménagés axialement dans les murs. Nord et Sud de т. Puisque les ébrasements présentent leur plus grande largeur du cỏté de la pièce à la meule, il est évident que celle-ci, dans un ètat antérieur des lieux, était contiguè à des pièces en partie ou en totalité hypèthres d'où lui venait la clarté du jour. On imagine difficilement que dans la dernière phase de $\omega$ les cuves en terre crue du cellier se soient trouvées à ciel ouvert.

2. Une réoccupation poncluelle et lardive, impossible à dater, a eu lieu en un endroit de la maison ou plutôt de ses ruines. Presque au sommet des décombres un muret a été construit en travers du passage $\tau / \omega$ (fig. 17). Sous trois assises de moellons les couches de destruction se superposent de façon chaotique, comme c'est le cas partout ailleurs dans le secteur Est : strates hérissées de pierres de construction (gneiss et pỏros), nids et traînées de bois carbonisé, lits de cendres, couches de terre et de cendre mèlées aux effondrements de stucs muraux, de pavement de sols, de mobilier d'étage. Le muret appuyé du còté Nord contre une épaisse coulée de mosaĩque de terrasse semble n'avoir été qu'un abri contre le vent ${ }^{25}$.

\section{Le plan de la Maison des Sceaux.}

Entièrement dégagée après la fouille du secteur Est, la Maison des Sceaux révèle un plan d'une remarquable clarté dont les parties paraissent correspondre aux différentes fonctions de l'habitation. Dès le vestibule la circulation se divise et s'oriente vers trois groupes de pièces (cf. $B C H 100$ [1976], p. 800, fig. 1) :

a) Celles du secteur Est desservies par le corridor v et séparées du reste de la maison. On vient de voir que leur destination proprement économique ne fait aucun doute.

b) Celles du rez-de-chaussée vers lesquelles conduit l'escalier descendant dans la cour $\theta$. On y reconnait la formule classique de l'habitat délien, avec notamment le groupe oecus major + desserte et avec des exèdres à larges baies.

c) Celles de l'étage desservies par l'escalier implanté contre le mur Sud de la cour. Le còté occidental, d'après la trouvaille des deux bustes-portraits dans les décombres de ، (BCH 99 [1975], p. 719) comportait un local d'apparat, peut-être une galerie articulée sur celle du Nord où était conservée la bibliothèque d'archives. A cette partie administrative des bureaux faisait suite, au-dessus du secteur Est, le groupe des chambres privees, des cuisines, des terrasses pour lesquelles on n'a pas identifié d'entrée indépendante. Il n'est pas impossible d'en restituer une à partir d'un palier de l'escalier Sud.

Cette architecture rationnelle commandait sans rigueur l'utilisation des locaux que pouvait imposer le quotidien. On a vu, par exemple, que des lots d'amphores étaient entreposés à l'étage et dans l'oecus major de la maison.

(34) Pour des modifications de ce type dans l'îlot des Bijoux : G. Sterert, «Un programme d'architecture privée à Délos", dans Le dessin d'archilecture dans les sociétés antiques (Actes du Colloque de Strasbourg, 1984), p. 175-185.

(35) D'autres réoccupations dans les ilots du quartier ont pu être datées par du matériel céramique de l'Antiquité tardive (BCH 90 [1966], p. 989 et 992-995). 


\title{
B. Cour de la Malson de l'épée (CD 59e)
}

\author{
par Françoise Alabe
}

Le but de cette campagne, qui s'est déroulée du 14 septembre au 9 octobre 1987, était d'achever le dégagement de la cour localisée en 1986 à l'Ouest de la Maison des sceaux (BCH 111 [1987], p. 629-644). Sous la direction de Fr. Alabe, l'équipe comprenait huit personnes, dont un ouvrier; Michel Rousset, assistant; Françoise Lefebvre-Rousset, restauratrice; Annick Chêle, Sabine Jaubert, Marie-Dominique .Nenna (membre de première année) et Annette Peignard.

\section{La cour de la Maison de L'épée (fig. 38).}

Les dimensions de la cour, presque rectangulaire, étaient connues dès 1986 (Ouest : 7,65 $\mathrm{m}$; Sud : 4,25 $\mathrm{m}$; Est : 7,60 m; Nord : $4,40 \mathrm{~m}$ ), mais seule une bande de $2,50 \mathrm{~m}$ au Sud avait été fouillée jusqu'au sol. La berme laissée en 1986 n'a pas été touchée en 1987, car son enlèvement aurait par trop fragilisé le palier de l'escalier de gneiss que nous n'avions pas les moyens de consolider convenablement dans le temps qui nous était imparti.

L'identification de cet espace, situé à l'Ouest de la pièce ı de la Maison des sceaux, comme étant la cour d'une nouvelle maison reposait sur trois critères ${ }^{26}$ :

- l'existence d'un sol dallé de gneiss, qui fait penser à un espace hypèthre;

- le décalage hypsométrique entre ce dallage $(11,57 \mathrm{~m})$ et le sol de ‘ $(10,96 \mathrm{~m})$ et de la Maison des sceaux dans son ensemble, sans qu'aucun dispositif d'escalier rattrapant ce décalage ait été observé lors du démontage de la cloison de pôros;

- le fait que cet espace ait un accès à la rue, via l'escalier de gneiss de l'angle Sud-Ouest.

A ces trois indices plaidant en faveur d'une cour d'habitation indépendante, la campagne de 1987 a permis d'ajouter la disposition des ouvertures dans le mur Nord (fig. 39a et 41) : la présence des fenêtres en particulier confirme l'hypothèse d'un espace à ciel ouvert. Mais les ouvertures vers l'Est et l'Ouest ont été bouchées avant la destruction, et dès lors, s'il s'agit bien d'une cour, force est de constater qu'elle ne joue que de manière réduite son rôle d'espace de circulation. Surtout, le fait que cet édifice ait été en cours de réaménagement lors de sa destruction définitive interdit d'être catégorique dans les conclusions ${ }^{37}$.

\section{LA FOUILLE.}

L'aspect général de la destruction a déjà été décrit à propos de la partie Sud : le caractère fragmentaire des décombres laisse rarement voir une cohérence, et favorise les éboulements, glissements et perturbations occasionnés par le travail des fouilleurs, mais aussi par d'autres causes. La découverte en plusieurs points de la fouille de squelettes de lapins (une dizaine en tout) fraichement nettoyés par les fourmis montre la grande perméabilité de la couche de destruction, une fois franchi le premier mètre où la terre est tassée par les précipitations, indurée par la sécheresse et maintenue par les racines des végétaux - mais aussi la perméabilité des couches qu'on serait tenté d'y distinguer. En l'occurrence, les lapins avaient traversé la bảche qui recouvrait verticalement l'arrêt de fouille 1986 ; des squelettes ont èté trouvés jusqu'à $2,50 \mathrm{~m}$ plus au Nord, à des niveaux qui s'étagent entre $1,50 \mathrm{~m}$ et $0,50 \mathrm{~m}$ au-dessus du dallage; presque à chaque fois, des fragments de la båche étaient en connexion avec les os $^{28}$; aucune trace du trajet que ces animaux avaient suivi à l'intérieur de la destruction n'apparaissait.

Faute de pouvoir utiliser la stratigraphie et les ensembles naturels pour l'enregistrement du matériel, nous avons opté pour un système de maillage de $0,50 \mathrm{~m}$ de côté, qui offrait un degrẻ de précision suffisant pour la

(36) Cf. G. Stebert, BCH 111 (1987), p. 632.

(37) Je remercie Ph. Fraisse de m'avoir fait bénéficier de ses observations et de ses doutes sur la «Maison de l'épée "; il conteste l'appellation de "maison", qu'il voudrait voir réservée aux cas où un projet architectural bien défini est décelable. Je conserve néanmoins ce terme au sens d'unité d'habitation indépendante, qui me paraít parfaitement adéquat à la nature de l'édifice tel qu'il se présentait au moment de la fouille.

(38) De sorte qu'on est en droit de se demander si ce n'est pas l'ingestion de cette båche qui a provoqué la mort des rongeurs autant que le comblement de leurs terriers. 


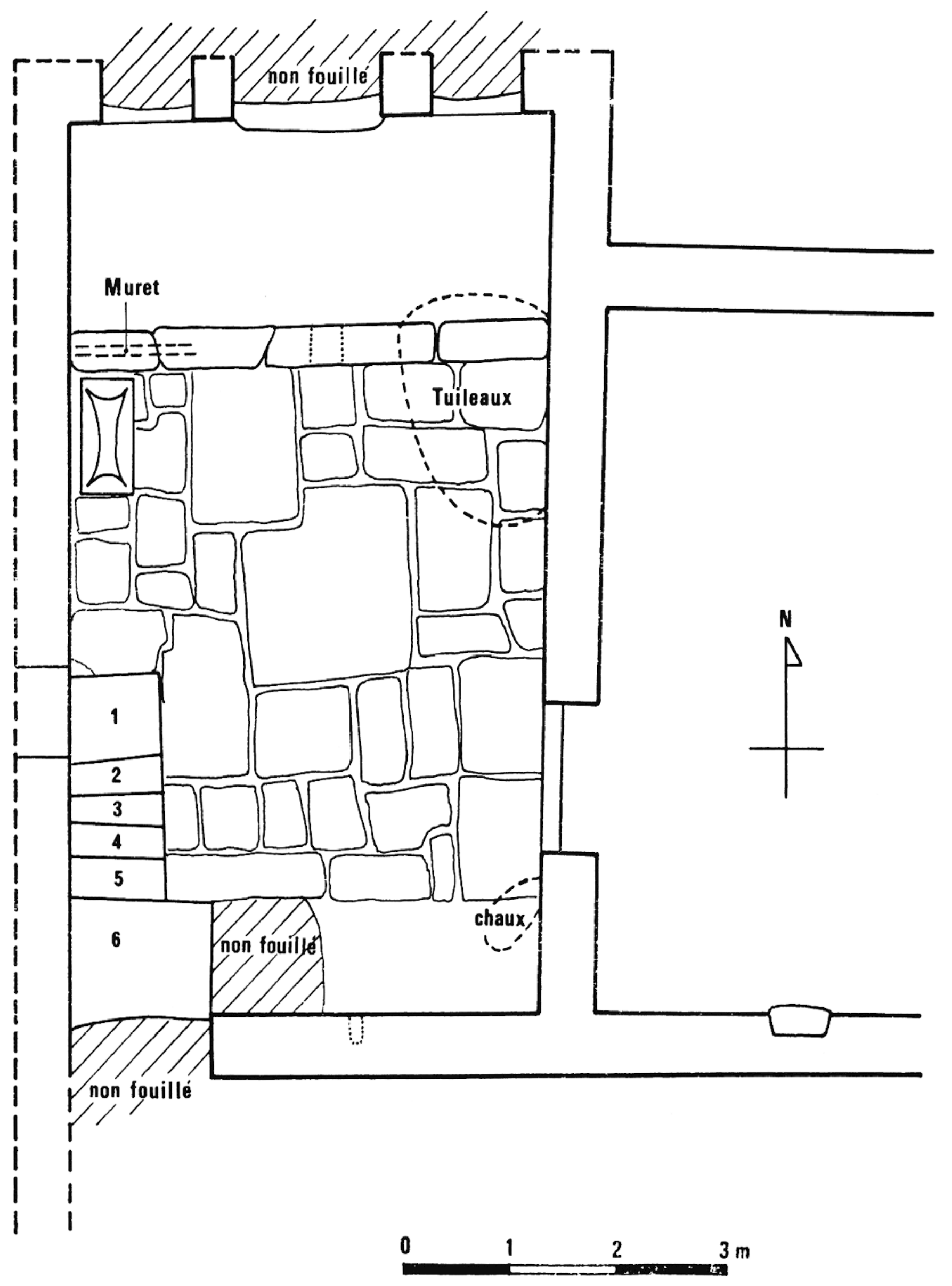

Fig. 38. - Cour de la Maison de l'Épée. D'après P. Abéla (1986). 
plupart des trouvailles, et permettait notamment d'envisager dans de bonnes conditions le traitement des fragments de décoration murale ou de ce que nous avions identifié l'an dernier comme des plafonds peints ${ }^{80}$, en nous fournissant d'emblée des micro-ensembles et en facilitant la recherche des collages. Chacune de ces mailles s'est vu attribuer un numéro allant de 01 à 78, le chiffre des unités croissant d'Ouest en Est et celui des dizaines du Nord vers le Sud. Le chiffre des centaines traduit notre progression verticale ${ }^{40}$ et correspond à des changements dans l'allure générale de la couche de destruction :

- une terre beige indurée infestée de pierrailles et de gros blocs de gneiss tombés de la partie haute des murs du rez-de-chaussée, avec revètement mural blanc (identique à celui resté en place), en miettes, concentré le long des murs, constitue la couche 500 ;

- à une hypsométrie moyenne de $13,20 \mathrm{~m}^{\mathbf{1 1}}$, soit environ $0,5 \mathrm{~m}$ en dessous de l'arrèt de fouille de 1986 , commençaient les décumbres d'élage, dans lesquels on a distingué une couche cendreuse (600) à gros blocs moins denses (jusqu'à une hypsométrie moyenne de $12,70 \mathrm{~m}$ ), et un niveau de démolition (700) caractérisé par une forte densité (un quart du cubage) en carreaux de póros et une grande fragmentation du mobilier (jusque vers $12,20 \mathrm{~m})$;

- enfin, une couche de destruction (800) a livré, aux côtés d'un matériel d'étage pulvérisé, des éléments importants relatifs à la vie du rez-de-chaussée (835/845 à 828/868 : tessons pilés, utilisés pour la préparation du mortier de tuileaux ; $840 / 850 / 841 / 851$ : reste de pan de bois hourdé; amphores $800 / 803 / 830 / 833$ ) et divers objets en place (fig. 40 et 43 );

- la pellicule charbonneuse et grasse, épaisse de 1 à $5 \mathrm{~cm}$, qui recouvrait le sol dallé de la partie centrale a reçu le n० 900 , mais son contenu est le même que celui de la couche 800 .

\section{TEChNique DE CONSTRUCTION, AMÉNAgement, DÉCORATION.}

La présence d'une restauratrice et le recours à des techniques de fouille minutieuses, adaptées au traitement des décors peints, ont permis de dégager environ $1 \mathrm{~m}^{2}$ (fig. 43) d'un type de construction peu fréquent ou, en tout cas, rarement mentionné et décrit, faisant intervenir le pisé sur colombage. Des poutres assemblées constituent la structure portante du mur ou de la cloison, et les vides laissés entre ces poutres sont remplis d'une terre sablonneuse mêlée de paille et de joncs, une couche de joncs liès en faisceaux, noyés dans la

(39) $B C H 111$ (1987), p. 643.

(40) La combinaison des trois numéros forme le numéro de sac; ainsi, les objets mobiliers trouvés au cours de la fouille ont été enregistrés sous la forme classique Millésime. $N^{\circ}$ de sac. $N^{\circ} d^{\prime o b j e l ~ d a n s ~ l e ~ s a c, ~ l e ~ n u m e ̀ r o ~ d e ~}$ sac donnant la situation de l'objet en fouille dans les trois dimensions. L'amphore 87.812.2 était dans la couche 800 et dans la maille $12(0,50 \mathrm{~m}$ à $1 \mathrm{~m}$ du mur Nord, $1 \mathrm{~m}$ à $1,50 \mathrm{~m}$ du mur Ouest); la lampe 87.570 .5 a été trouvée intacte dans la couche 500 , dans la maille 70 (soit $3,50 \mathrm{~m}$ à $4 \mathrm{~m}$ du mur Nord et 0 à $0,50 \mathrm{~m}$ du mur Ouest). Ce système d'enregistrement permet de juger si un objet est "en connexion " ou, au contraire, "éclaté * : la lampe 87.570.5 était prise en sandwich entre les tessons d'une amphore, $n^{\circ} 87.570 .41$, complétée par des tessons 87.560.117, c'est-à-dire dans la même couche et dans la maille voisine au Nord - il s'agit bien d'une connexion; en revanche, des fragments d'un même askos sont répartis dans les sacs $\mathbf{n}^{08} 87.631,635,654,733$, $734,736,743,816,817,818,834,835,836,852,877$ - ce qui indique à la fois que les couches 600,700 et $800 \mathrm{ne}$ sont pas étanches, et que certains objets se sont brisés et dispersés sur une aire qui peut étre assez vaste avant la chute des murs (les fragments manquants de cet askos, encore incomplet, ont peut-ètre été trouvés en 1986, à moins qu'ils n'aient rebondi jusque dans les pièces voisines). Un vase reconstitué à partir de tessons enregistrés dans des sacs différents porte le premier numéro de sac et d'objet dans l'ordre arithmétique.

(41) Je parle de niveaux moyens car les couches ne sont, bien entendu, pas planes ; la fouille s'était arrêtée, en 1986, à environ $13,70 \mathrm{~m}$ (avec un plongeon d'environ $15 \mathrm{~cm}$ dans les angles Nord-Est et surtout Nord-Ouest, et un début d'éboulement au Sud malgré la palissade de madriers). Le point le plus haut de ce qui correspond à la couche 87.600 avait èté atteint, en 1986, à 13,90 m (cf. $B C H 111$ [1987], p. 632); il est évident que ce "sommet" était dù à la présence de l'escalier de gneiss, qui a rehaussé artificiellement les décombres d'ètage. Pour le secteur fouillé en 1987, les couches 600 et 700 étaient pratiquement absentes le long du mur Nord, en raison de l'épaisseur de la couche 800 (éléments en place du rez-de-chaussée : le massif d'amphores de l'angle Nord-Ouest, mentionné ci-après p. 776-778) et du tassement de la couche 500 (haut du rez-de-chaussée) vers le Nord-Est. Enfin, le mélange de gros matériel lourd (gouttières en plomb, moellons) et de matériau friable (mortier d'enduit mural réduit en poussière) provoque un micro-vallonnement incessant, toujours instable et qui ne présente pas de signification réelle. 


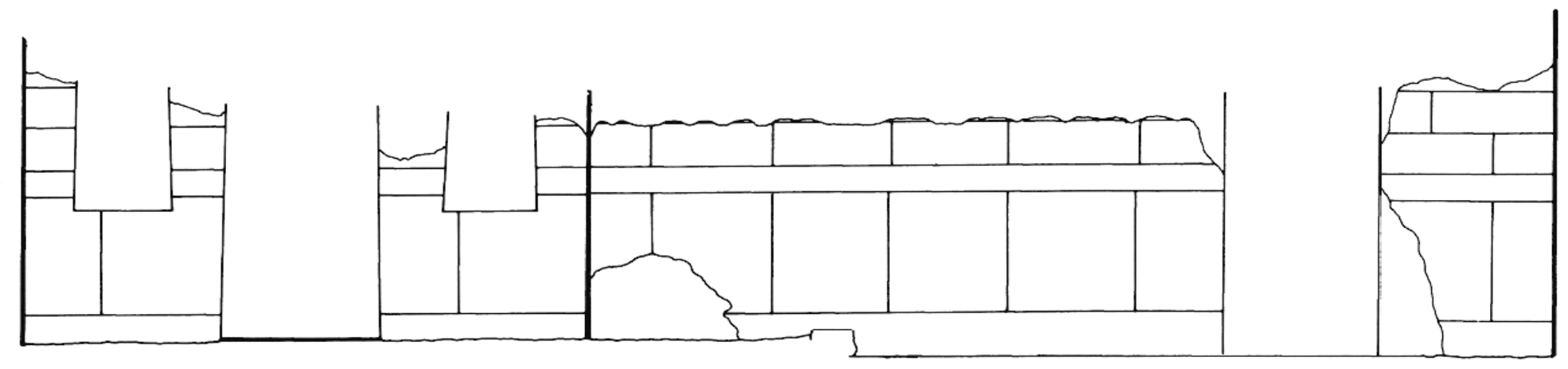

a : les murs Nord et Est.

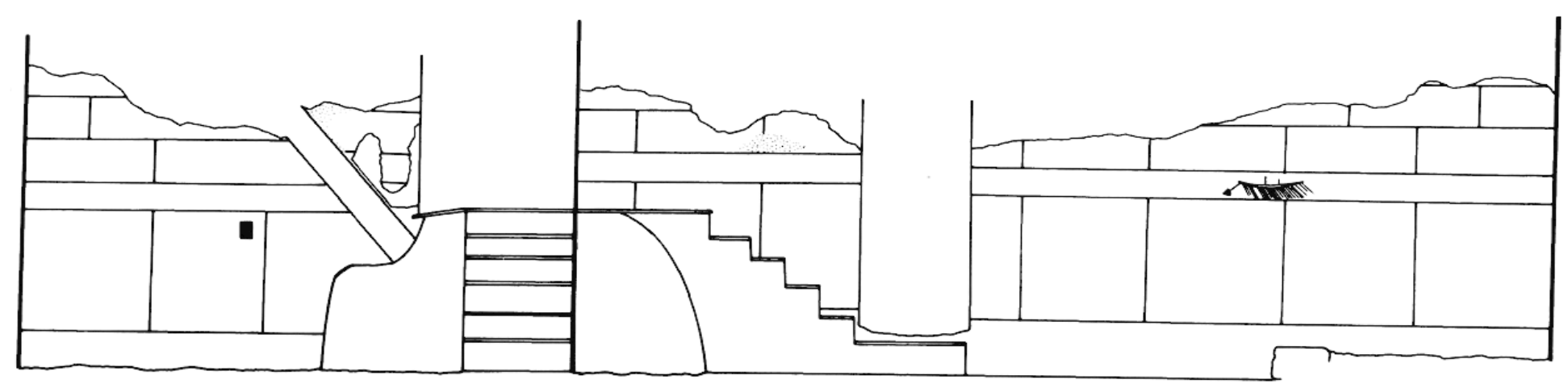

b : les murs Sud et Ouest.

Fig. 39. - Revêtement mural en place.

Relevé : P. Abéla/Fr. Alabe (1986), Fr. Alabe/Fr. Lefebvre-Rousset (1987); dessin : Fr. Alabe. 
même terre, égalisant le tout : ce pisé avait été reconnu en 1986 au dos de fragments d'enduits, mais à trop petite échelle pour que la structure de la maçonnerie soit observable. Nos efforts de consolidation ${ }^{42}$ n'ont pu prolonger la vie de cette structure, d'une extrème fragilité, que le temps de procéder au relevé.

Etant donné la nature des peintures observées sur pisé en $1986^{43}$ (une corniche, une peinture murale d'étage et un plafond), on est en droit de penser que cette architecture légère était employée surtout en partie haute (couronnement de mur, cloisons d'ètages, éventuellement plafond); mais le grand fragment observé cette année semble enduit sur ses deux faces et doit donc provenir d'une cloison. D'autre part, le point d'appui de cette structure sur le mur Ouest correspond à l'extrémité Ouest d'une tache noire de forme rectangulaire qui s'étend sur les murs Nord et Ouest (hauteur : $1 \mathrm{~m}$; largeur sur le mur. Nord : $1 \mathrm{~m}$; sur le mur Ouest : $2 \mathrm{~m}$, non compris le prolongement en pointe d'environ $0,90 \mathrm{~m}$; cf. fig. 41 et 42). Les fragments d'une amphore écrasée en place reposaient immédiatement sur la conjonction de poutres et de pisé décrite ici, tandis qu'une douzaine d'autres étaient rangées à l'horizontale dans l'angle Nord-Ouest de la cour, écrasées sur plusieurs niveaux et mélangées à de très nombreux fragments de bois brúlé et à des clous dont certains sont restés collés aux amphores. La structure de pan de bois hourdé doit dès lors s'interpréter comme la limite Ouest d'un "casier à amphores» en bois, d'un type déjà attesté hors de Délos" ; elle s'est déversée vers l'Ouest sous le poids des amphores qu'elle contenait, lors de l'incendie, mais devait être fondée sur l'emmarchement de gneiss qui délimite une terrasse de terre battue au Nord de la cour.

Le dallage de gneiss ne s'étend pas, en effet, sur toute la superficie de la cour. En 1986, on avait observé que les dalles faisaient place, au droit de l'escalier menant à la rue, à un sol de terre battue (larg. $1 \mathrm{~m}$ ) le long du mur Sud. A $2 \mathrm{~m}$ en avant du mur Nord, la fouille de 1987 a mis en évidence un emmarchement de gneiss (haut. $0,25 \mathrm{~m}$ ) donnant accès à une petite terrasse au sol également de terre battue. Selon une disposition fréquente à Délos dans les cours de maisons d'habitation, le mur Nord est percé d'une porte centrale (larg. 1,24 m) et de deux fenêtres symétriques (larg. $0,80 \mathrm{~m}$ et $0,76 \mathrm{~m}$; haut. d'appui $1,10 \mathrm{~m}$ ). La pièce (non fouillée) située au Nord de ce mur répondrait bien à la la configuration habituelle de l'oikos, largement ouvert au Sud (les ouvertures occupent près des deux tiers du mur) et prenant jour sur la cour par l'entremise d'un espace couvert mais non fermé. La disposition d'ensemble de ce secteur Nord - porte et fenêtres, terrasse légèrement surélevée (fig. 41) - tend donc à confirmer l'identification de la zone fouillée en 1986 et 1987 comme cour d'une nouvelle habitation.

On n'a pas vérifié par sondage si l'emmarchement de gneiss et le sol de terre battue avaient été posés sur le dallage dans le cadre d'un remaniement, ou si l'ensemble était originel. En fait, le sondage réalisé en 1986 dans l'angle Sud-Est de la cour, sous le sol de terre battue, a établi que le revêtement mural en place est contemporain de l'installation du dallage, et que les deux coincident avec la construction du mur Est. Or, on observe que le même revêtement enjambe l'emmarchement et épouse le sol de la terrasse (il s'arrête même quelques $\mathrm{cm}$ au-dessus, l'adhérence entre le mortier et la terre ayant joué avec le temps). Il est donc certain que l'ensemble a été réalisé en une seule opération, ce qui peut expliquer la hauteur inhabituelle de la plinthe dans la partie Sud : le tracé de l'incision tenait évidemment compte du niveau du sol le plus haut (précaution analogue à celle qu'on remarque, par exemple, dans le vestibule de la Maison des Masques).

(42) L'ensemble est apparu le $1^{\text {er }}$ octobre en fin de matinée. Fr. Lefebvre-Rousset et A. Peignard en ont poursuivi le dégagement au scalpel et au pinceau, en l'imprégnant de Paraloĩd B72 dilué à $5 \%$ dans l'acétone, jusqu'au soir; l'humidité nocturne a perturbé le séchage du Paraloĩd, et la pluie a commencé le 2 octobre sans qu'une dépose soit envisageable; il ne restait plus qu'à garder une trace graphique de cet objet voué à disparaitre.

(43) Cf. $B C H 111$ (1987), p. 631 (fig. 5-6); $632 ; 641$ (fig. 21); 643.

(44) L'exemple le mieux conservé qu'on en connaisse est la caupona $(\mathrm{V}, 6)$ d'Herculanum, attenante à la Maison de Neptune et d'Amphitrite (visible depuis le cardo IV; cf. A. MaIuri, Herculaneum, série Ilinéraires des musées et monuments d'Italie, Rome [Ministère de l'instruction publique], $5^{e}$ édition, 1976, p. 44-45 et p. 115, pl. XXVII fig. 45-46) : des planches disposées de chant sont évidées, à intervalles réguliers, d'arcs de cercle formant berceaux dans lesquels reposent les amphores; l'ensemble de ces étagères s'insère dans l'armature d'une soupente, en bois elle aussi. Je restitue ici un dispositif analogue, mais posé au sol et pris entre le mur Nord et le muret à pan de bois hourdé dont la hauteur ne devait guère dépasser $1,50 \mathrm{~m}$. Le choix de ce matériau s'explique-t-il par la difficulté de se procurer du bois à Délos, ou (ce qui me paraitrait plus vraisemblable) par le désir de protéger plus efficacement une réserve de vin d'un certain prix? On manquera sans doute longtemps d'élèments pour en décider. 
Fait assez rare pour être noté, la cour ne comporte pas d'embouchure de puits ni de citerne. D'importants fragments de gouttières en plomb ont cependant été recueillis dans la destruction (le long du mur Est, près de l'arrêt de fouille 1986; du côté Ouest, environ $1 \mathrm{~m}$ en avant de l'emmarchement de gneiss : fig. 5), attestant l'existence d'un dispositif de collecte des eaux de pluie. D'autre part, un caniveau descendant vers le . Vord passe sous la terrasse à peu près en son milieu (fig. 41). Une vasque rectangulaire de marbre blanc (larg. $0,55 \mathrm{~m} ;$ long. $1,08 \mathrm{~m}$; haut. $0,35 \mathrm{~m}$; prof. $0,18 \mathrm{~m}$; fig. 42) reposait sur le dallage dans l'angle Nord-Ouest.

Il n'y avait aucun vestige ni trace de colonnade ou de péristyle. La terrasse .Nord ne pouvait donc étre abritée que par un auvent en matériaux légers (voire un simple couvert végétal).

La maison a connu au moins un remaniement avant l'ètat actuellement visible (sur le mur Est, près de l'angle Nord-Est, la chute du revètement mural laisse voir un état antérieur, blanc uni : cf. fig. 39a). Mais surtout, l'observation du revêtement mural en place (fig. 39; description du système BCH 111 [1987], p. 642), presque partout bien conservé au moment de la fouille, et la présence de deux tas de matériaux de constructions le long du mur est, obligent à penser que la destruction violente de la maison en 69 a empèché l'achèvement de travaux en cours.

On s'interrogeait en effet sur l'interruption du revêtement du mur Ouest à l'aplomb de la première marche de l'escalier (fig. 39) : la largeur de cet arrêt $(0,84 \mathrm{~m})$ et le fait que ses lignes verticales correspondent à des * coups de sabre dans la maçonnerie donnaient à penser qu'il s'agissait d'une porte murée, sans doute lors d'un remaniement important ou à la suite d'une destruction partielle puisqu'il n'y a pas de linteau et que l'appareil est perturbé, au-dessus des coups de sabre, sur une largeur d'au moins $2 \mathrm{~m}$ (fig. 42 et $B C H$ 111 [1987], p. 634 fig. 9); mais rien ne justifiait que ce bouchage ait èté laissé visible au lieu d'être recouvert d'un enduit. Normalement, toute réfection du mur entraîne la réfection immédiate du revètement mural, qui masque les irrégularités de la construction.

D'un autre cóté, la présence d'une porte à cet endroit peut seule expliquer la forme carrée de la marche inférieure de l'escalier : cette marche devait se monter et se descendre aussi bien dans le sens Nord-Sud (accès au vestibule et à la rue) que dans le sens Est-Ouest (accès aux pièces desservies par la porte, murée tardivement). L'arrêt inférieur du revêtement, détruit sur la largeur de la porte, est cependant conservé aux angles, presque au niveau de la deuxième marche de l'escalier; ce petit fait assure la cohérence de l'ensemble : il y avait bien une porte, surélevée de deux marches par rapport à la cour.

La seule hypothèse qui rende compte à la fois de l'existence ancienne d'une porte liée à l'escalier, de son bouchage et de l'absence d'enduit mural sur ce bouchage, est que la maison fut détruite pendant des travaux de réaménagement, après la phase du gros œuvre et avant l'achèvement des finitions. Aussi bien le tas de tessons pilés (environ 1,5 $\mathrm{m}^{2}$; fg. 40) signalé plus haut, que la chaux trouvée en 1986 près de l'angle Sud-Est étaient, sans nul doute, destinés à la rénovation des enduits dans la cour ou dans l'habitation en général.

Le fait que ces travaux de réfection des peintures aient été déjà commencés, et mème près de leur terme, lors de la catastrophe finale, est indiqué par l'état du revêtement en place sur le mur Sud de la cour (fig. 395). Dans l'Antiquité comme de nos jours, les travaux de finition des murs progressent normalement du haut vers le bas. Il est donc normal que l'on ait trouvé le revêtement piqueté et recouvert d'une nouvelle couche d'enduit vers l'angle Sud-Ouest, au-dessus de l'escalier de bois qui menait à l'étage, et piqueté sans nouvel enduit sur le reste du mur Sud, au-dessous de cet escalier; le mur Sud était, dans l'ordre normal d'exécution des travaux, à la fois le dernier mur de l'étage (partie haute) et le premier du rez-de-chaussée. Aussi les trois autres murs de la cour sont-ils restés intacts : on n'eut pas le temps de les commencer.

La remise à neuf de la cour, interrompue par la destruction, est donc la phase finale de travaux ayant affecté au moins en partie le reste de la maison, notamment l'étage. La très belle décoration murale apparentée au second style pompéien, dont des fragments découverts en 1986 ont été remontés sur panneau à l'automne $1987^{45}$, a du ètre réalisée très peu de temps auparavant, lors de ces mêmes travaux; il est d'un grand intérêt pour l'histoire de la peinture antique de disposer d'une datation aussi précise.

Des graffites ont été trouvés cette année : graffite de bateau (fig. 44 et $39 \mathrm{~b}$ ) en place sur le mur Ouest; rosace au compas inachevée, et dessin répété 4 fois d'un objet non reconnaissable, en fragments dans la couche de destruction (près du mur Ouest, au voisinage du bateau; 87.540); la nature du mortier indique que ces fragments sont très probablement tombés du mur Ouest.

(45) Cf. BCH 111 (1987), p. 643. Dominique Deyber et Cécile Maquet ont procédé aux essais techniques, tests de compatibilité et choix des produits, durant l'èté ; après le départ de C. Maquet, Fr. Lefebvre-Rousset a aidé $\mathrm{D}$. Deyber dans la réalisation finale du panneau. 
Fig. 40. - Partie Est de la couche 800 : tessons pilés à cheval sur l'emmarchement de gneiss (trace claire sur le mur et granulométrie régulière), amphore 87.844.6, cuvette en marbre 87.853.1, fragments des amphores 87.866.4 et (col) 87.864.2. Photo :

Fr. Alabe, EFA.

Fig. 41. - Fin de la fouille, vue vers le mur Nord. Photo: Fr. Alabe, EFA.

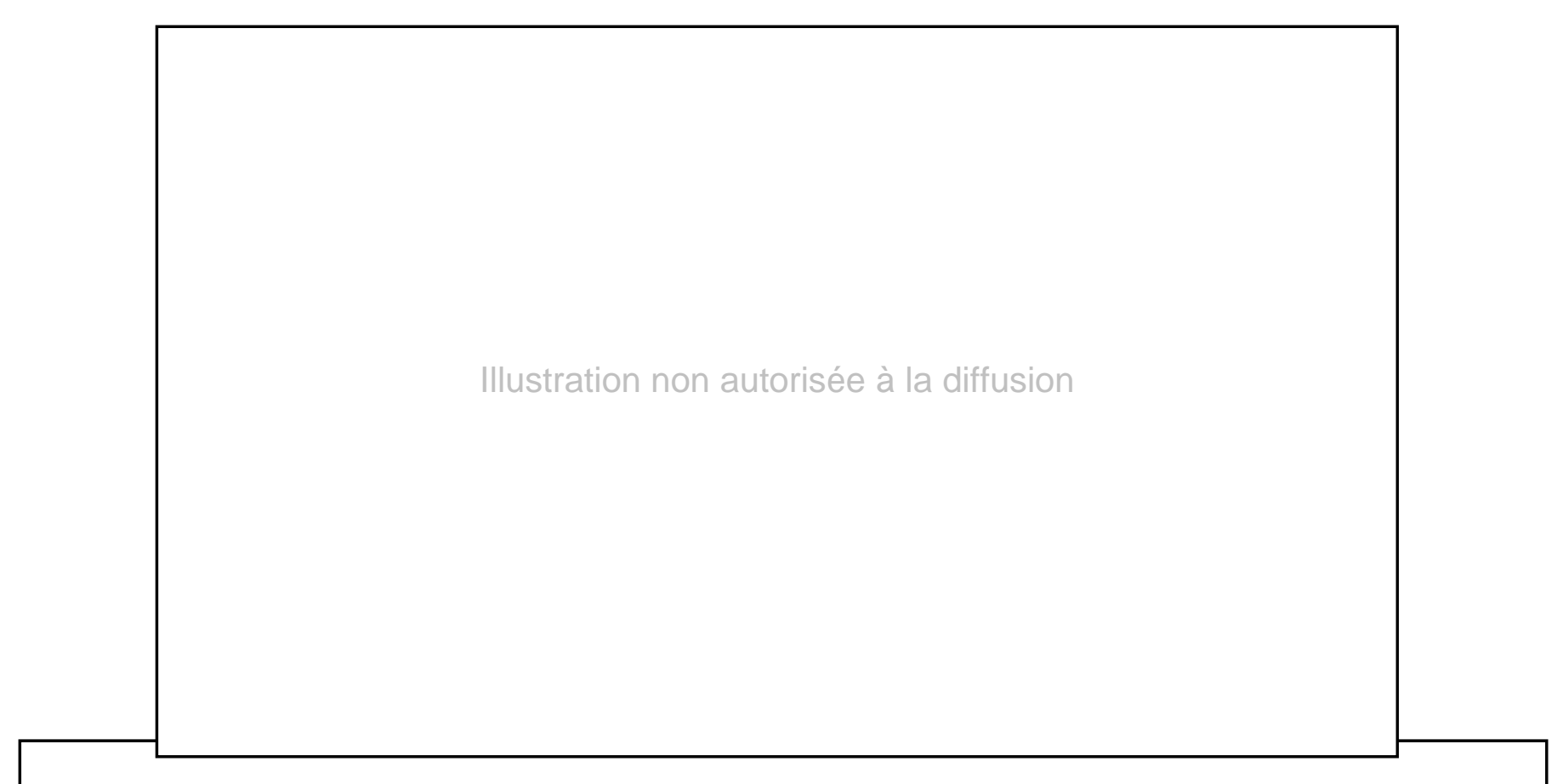

Fig. 42. - Fin de la fouille, vue vers le mur Ouest (partie Nord); au premier plan, couche 900 , gouttière 87.951.1. Photo : Fr. Alabe, EFA. 
Fig. 43. - Mailles 840 et 850, pan de bois hourdé. Relevé : A. Peignard/Fr. Lefebvre-Rousset; dessin : Fr. Lefebvre-Rousset.

Fig. 44. - Graffite de bateau en place sur le mur Ouest. Relevé : Fr. Alabe/M. Rousset. 


\section{Revêtements MURAUX ET DISPOSITION DE L'ÉTAGE.}

L'étude des fragments sera complétée prochainement, mais les premières observations sont déjà partiellement interprétables. Tout d'abord, les deux ensembles remarquables identifiés en 1986 étaient presque absents du secteur fouillé en 1987; les rares fragments de décoration du Second style et de plafond peint ne se rencontraient qu'aux abords de l'arrêt de fouille : il est probable que ces deux décors étaient associés dans une même pièce, située au Sud (peut-être au Sud-Est) de la cour.

L'ensemble du Premier style, banal mais soigné, qui abondait en 1986 est, lui aussi, très peu représentè en 1987 ; il semble provenir d'une pièce située plutôt au Sud-Ouest to.

Les fragments de ces trois ensembles se raréfiant aux approches du sol, il est plausible que ces deux pièces sont tombées après le reste de l'étage. Cette hypothèse est renforcée par la taille des fragments et l'étendue des connexions, respectivement en 1986 et 1987 : le matériel de 1987 est littéralement pulvérisé, surtout dans le dernier mètre de la couche de destruction, et se prête mal au remontage en bac à sable de panneaux importants comme c'était le cas l'année précédente.

Un nouveau décor a fait son apparition en 1987, dans les couches 700 et 800 . Bien que la petite taille des fragments empêche toute observation poussée, l'ensemble paraît très simple et d'un type banal : orthostates à incisions simples et filets d'encadrement contrastés, bandeau uni ou à couleurs plates alternées (couleurs très altérées par l'incendie, autour d'une gamme d'ocres), sans galons de transition.

Enfin, quelques fragments trouvés près du mur Nord, qui se distinguent des revêtements du reste de la maison par des motifs légèrement différents, d'un plus grand module, et par l'usage du relief stuqué, proviennent sans doute de l'oikos voisin (un fragment a été trouvé en nettoyant le seuil de cette pièce).

A une seule exception près (une lampe intacte à deux becs ornés de masques satyriques, 87.570.5), la céramique était très fragmentaire. Une autre lampe a retenu l'attention après recollage, car le type n'en a pas été signalé à Délos. Il s'agit d'un bilychne tourné (un seul des deux becs a servi) à tuyau central sur lequel coulisse un couvercle"?

Le matériel de rez-de-chaussée est essentiellement constitué d'amphores brisées en place, dont la plupart, sans doute stockées à l'horizontale sur la terrasse Nord, ont été trouvées encastrées les unes dans les autres mais d'autres amphores ètaient rangées en d'autres endroits de la cour (cf. BCH 111 [1987], p. 635 fig. 12, ou ici fig. 40).

C'est en tout cas dans le casier que se trouvaient deux amphores identiques, de type Dressel IB, portant le mème dipinto en latin à l'ocre rouge et mentionnant un nom de consul, indice qu'elles contenaient un vin réputé et millésimé (fig. $45 a$ et b).

Pour le reste, le matériel ne se différencie guère de celui de l'an dernier que par des absences ${ }^{\text {* }}$. La quantité de vaisselle fine paraît très faible, en nombre de tessons et surtout en nombre de vases complets ou laissant bien augurer des campagnes de recollage prévues. Je mentionne ici une cruche d'une fabrique et d'un type qui me paraissent peu familiers (fig. 46) - je n'en ai pas vu de parallèle au Musée de Délos. La pâte, fine et très dure, a la couleur et la granulométrie des céramiques corinthiennes; sa dureté ne l'empéche pas d'ètre très poreuse, et l'aspect du vase en a beaucoup souffert, puisque les éléments étrangers (charbon de bois, plomb oxydé, clous de fer oxydés) ont taché la pâte en profondeur - et de manière variable, puisque les tessons étaient répartis sur un rayon d'1 $\mathrm{m}$ autour de la vasque de marbre (cette portion de la couche 900 est visible fig. 42). L'excellente qualité de la pâte et de la cuisson contraste avec l'aspect négligé de la fabrication proprement dite : la forme est peu élaborée (panse biconique, goulot à peine resserré, lèvre étalée sans bec) et s'accorde bien avec le tournage hâtif que dénote le fond plat, à peine lissé et démaigri extérieurement, très épais ; l'anse, mal collée, s'est séparée du vase sans presque laisser de trace (elle doit se trouver, avec les quelques éclats manquants de la panse, sous la vasque en marbre); enfin, le vase a été manié sans précaution avant la fin du séchage, ce qui l'a déséquilibré et

(46) La campagne de restauration de 1987 n'a presque pas porté sur cet ensemble, dont le traitement a été réservé pour 1988. Aux dimensions données $B C H 111$ (1987), p. 643, le travail d'Amalia Ghaki, Sophie Reynard et Véronique Rossignol permet d'ajouter la hauteur de l'orthostate $: 87,5 \mathrm{~cm}$. $B C H$.

(47) Cette lampe, ainsi que d'autres fragments se rattachant au mème type, sera publiée dans un prochain

(48) Ainsi, la mosaĩque décorée semble cantonnée au Sud de la cour, comme (ou avec?) la peinture du Second style; il en est de même pour le riche mobilier métallique (on n'a trouvé que quelques fragments de cadre de lit, adhérents au bois carbonisé qu'ils plaquaient). 

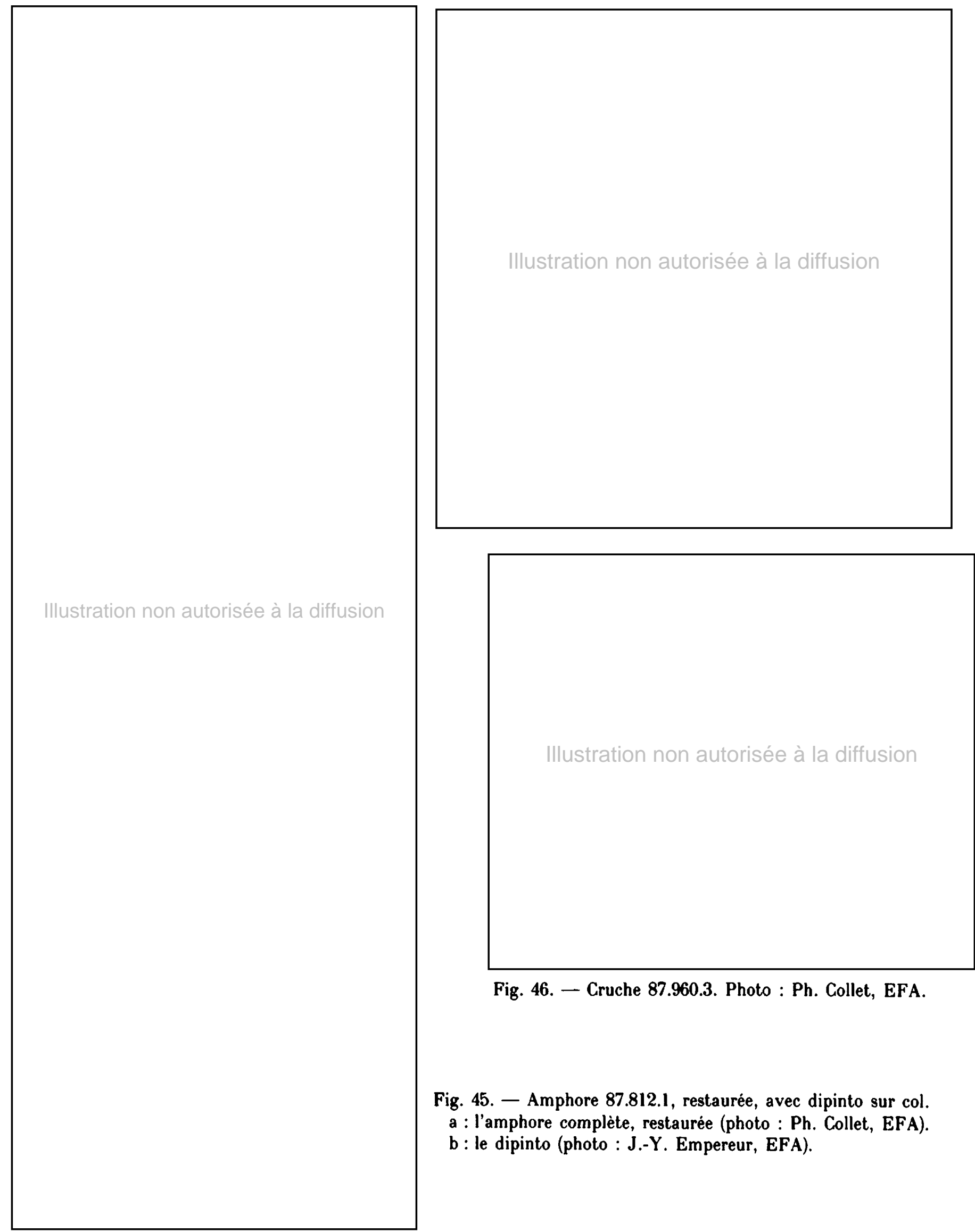

Fig. 46. - Cruche 87.960.3. Photo : Ph. Collet, EFA.

Fig. 45. - Amphore 87.812.1, restaurée, avec dipinto sur col. a : l'amphore complète, restaurée (photo: $\mathrm{Ph}$. Collet, EFA). b : le dipinto (photo : J.-Y. Empereur, EFA). 
l'a laissé couvert de traces de doigts imprégnés de barbotine. A cause de l'association, sur une mème pièce, de qualités techniques évidentes et de défauts techniques non moins évidents, je rangerais ce vase dans la catégorie des "pseudo-fines".

Le matériel d'étage, plus varié (mais comportant aussi des amphores), a subi une très forte dispersion dès ou même avant le début de l'incendie, qui n'a pas eu partout la mème violence : très souvent, les tessons jointifs d'un même vase, non seulement ont été trouvés en des points éloignés les uns des autres, mais ne présentent pas les mêmes traces d'incendie (c'est le cas de l'askos mentionné supra, note 40). On retrouve les mêmes catégories, et souvent les mêmes objets, qu'en 1986; malgré sa brièveté, la campagne de recollages du printemps a permis d'entamer le remontage de quelques vases à partir du regroupement de tessons de 1986 et 1987; parmi ceux-ci, trois lagynoi $(87.570 .11 ; 87.745 .14 ; 87.856 .6)$ ont déjà été partiellement complétés par des tessons trouvés en 1986; quelques pièces de vaisselle utilitaire (vase en pâte très friable, de piètre qualité, décoré de feuilles, 87.870.17; casseroles 87.540 .65 et 87.838 .22 ) démontrent que les rapprochements pourraient être poursuivis avec succès sur un matériel très courant, mais finalement méconnu.

Des fragments de pots à deux anses en ruban verticales, fond bombé et lèvre à gorge intérieure remplie de plâtre (cf. $B C H 111$ [1987], p. 633) ont été découverts en très grand nombre sur l'ensemble de la fouille et dans toutes les couches. Leur abondance impose qu'on leur porte intérêt, mais complique singulièrement la tâche, et l'on ne dispose pas encore de suffisamment de formes complètes pour savoir, par exemple, si le profil et le module sont aussi réguliers qu'on en a l'impression à première vue, à combien d'exemplaires correspondent les centaines de tessons rattachés à cette forme sur un espace fouillé assez restreint, ni surtout avoir une idée de leur contenu et de leur usage.

\section{Conclusion provisoire.}

L'histoire de la maison avait été ébauchèe à l'issue de la campagne 1986 (BCH 111 [1987], p. 641-642) selon un schéma en trois phases. Il est possible d'apporter quelques précisions sur ce processus.

1. La première phase correspond à un état où la distribution de l'espace est très différente de l'état actuẹl; la pièce ‘ de la Maison des sceaux se prolonge vers l'Est sans solution de continuité, occupant une partie de la cour voisine fouillée en 1986-87. La limite Sud de cet ensemble devait être le mur Sud actuellement commun à ı et à la cour de la Maison de l'épée, sa limite Ouest est inconnue, et sa limite Nord était peut-être dans le prolongement de l'actuel mur Nord de $\iota:$ on a relevé, près de l'extrémité Nord du mur Est de la cour, les traces d'un état plus ancien du revêtement mural (cf. fig. 39a).

2. Le mur Est de la cour de la Maison de l'épée, dont la construction a été reconnue contemporaine de l'installation du dallage par un sondage dans l'angle Sud-Est, n'est donc pas d'une seule venue. Toute communication avec la Maison des sceaux ayant été bouchée par la cloison de pôros, la cour était accessible de la rue par l'escalier Sud-Ouest, et donnait accès par le Sud à l'étage, vers le Nord à une pièce qui serait l'oikos, et vers l'Ouest à une ou des pièces dont nous ne savons rien.

3. Après une destruction partielle du mur Ouest, des travaux entrepris dans la Maison de l'épée furent définitivement interrompus par l'incendie qui détruisit aussi la Maison des sceaux en 69. Ces travaux de réaménagement avaient une certaine ampleur, puisqu'on a trace matérielle d'une réfection de peintures à l'étage, et du bouchage de la porte Ouest; l'absence de linteau au-dessus de cette porte invite à se demander si la destruction partielle du mur Ouest ne fut pas accidentelle.

Il n'est pas sans intérêt, peut-être, de noter que la maison restait habitée pendant les travaux, fût-ce au prix de dispositifs temporaires. La cour, déjà exiguë, était surencombrée d'objets divers qui y étaient déposés, pour la plupart, de façon provisuire, et l'accès à l'oikos Nord était rétréci aussi bien par la présence des matériaux de construction que par le casier à amphores de l'angle Nord-Ouest. La fouille a donc saisi la Maison de l'épée en pleine phase de transition, rendue définitive par la catastrophe finale. 


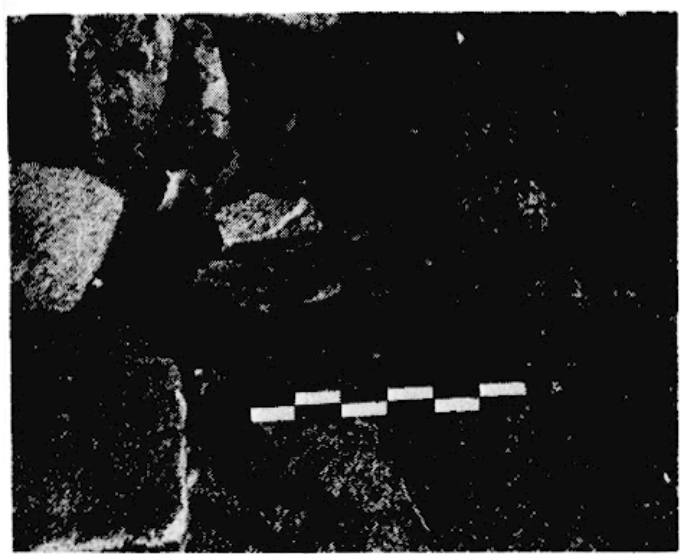

Fig. 47. - Fondation dans l'angle Sud-Ouest de la skènè (du Sud).

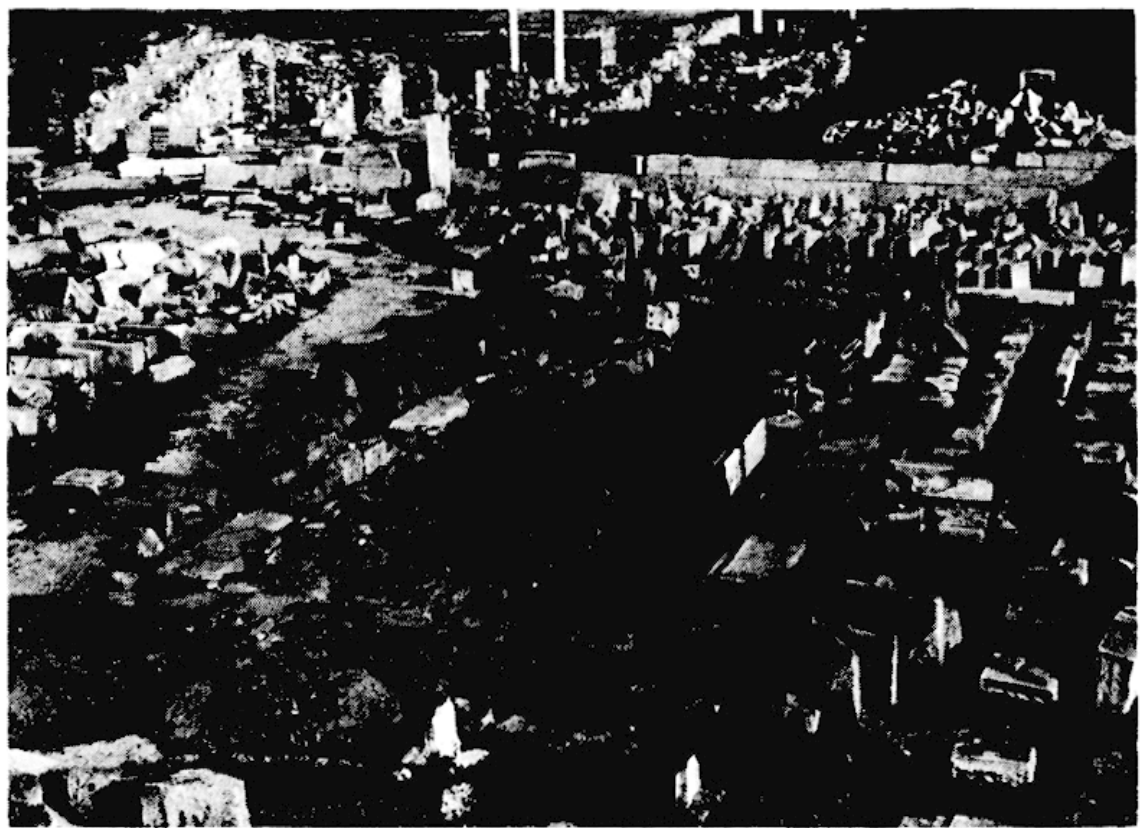

Fig. 48. - Vue générale des travaux dans le båtiment de scène (du Sud).

\section{3. - Le theatre}

par Jean-Charles Moretri

Les travaux se sont déroulés du 6 au 16 avril avec une équipe de deux ouvriers, sous la direction de J.-Ch. Moretti. Ph. Fraisse a effectué les relevés de fouilles.

Les blocs errant dans le bâtiment de scène ont èté systématiquement rangés. Cette mise en ordre a permis de dégager dans l'angle Sud-Ouest de la skènè la fondation (fig. 47) que R. Vallois proposait d'interpréter comme "le reste d'une des fontaines (xpouvoi) citées $I G$ XI 2, 287, A, I. 79"49. Sise à 4,25 m au Sud de l'axe EstOuest de la skènè, elle comporte, sur une longueur de $0,93 \mathrm{~m}$, une seule assise d'un double parement de petits moellons, qui vient buter contre le mur Ouest de la skènè. Le rocher n'a conservé aucune trace qui permette d'en compléter le plan. Il est néanmoins peu probable qu'il s'agisse des vestiges d'une fontaine puisqu'aucune installation hydraulique n'a été repérée dans le secteur. Selon que l'on considère que la fondation a entièrement conservé ou non son extension, on songera soit au socle d'un escalier de bois accolé au mur Ouest de !a skènè ${ }^{50}$,

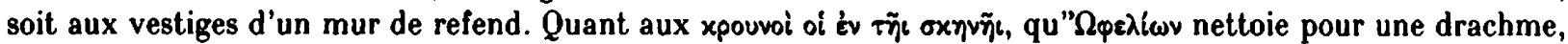
au mois d'Arésion de 250 av. J.-C., nous sommes tenté de les interpréter commé des sections des caniveaux qui menaient les eaux de l'orchestra vers la citerne aménagée à l'Ouest du théâtre. La branche Nord du canal passe, en effet, au pied de la stoa septentrionale de la skènè, celle du Sud en traverse la stoa méridionale. Ces xpouvoi de

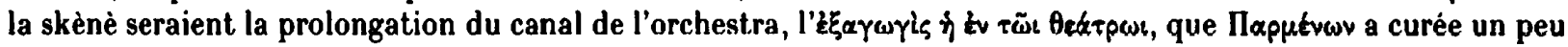
plus tôt cette mème année 250 , au mois de Hiéros, pour la somme de deux drachmes (IG XI 2, 287, A, I. 50).

(49) R. Vallors, $A H H D$, I, p. 225, n. 1. Cf. aussi o.c., p. 265.

(50) Des xגifuxxę de bois sont mentionnées dans les comptes du théâtre (IG XI 2, 199, A, I. 95 et 203, A, 1. 43), mais elles peuvent aussi bien avoir servi d'escaliers ou d'échelles entre les différents niveaux de la skènè que d'accessoires pour les représentations.

Sur les escaliers en bois à Délos cf. J. Chamonard, EAD, Vili 2, p. 307-308 et Ph. Bruneau et alii, $E A D, X X V I I$, p. 43 et 47 . D'autres socles de pierre pour escaliers de bois sont connus à Olynthe, Cassopè et Thasos, cf. D. M. Robinson, J. W. Graham, Olynthus, VIII (1938), p. 271-273; W. Hoepfoner, E.-L. Schwandner, Haus und Stadl im klassischen Griechenland (1986), p. 57 et 110; Y. Granjean, ElThas., XII (1988), p. 408. 


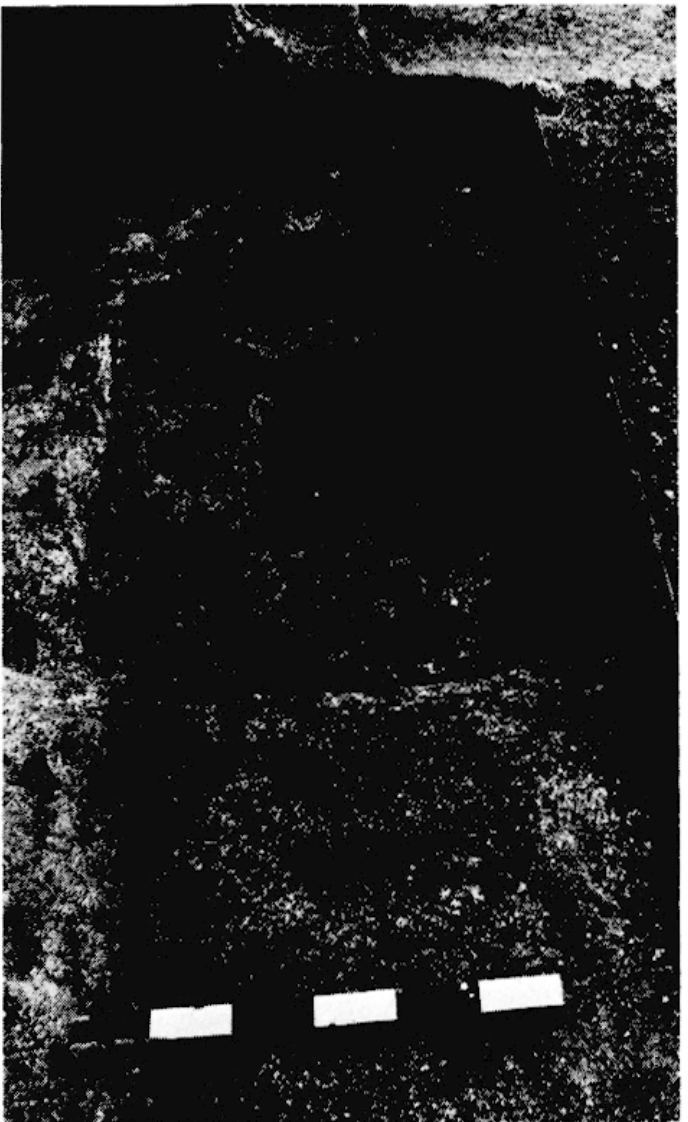

Fig. 49. - La fosse de la stoa Ouest (de l'Ouest).

Fig. 50. - La fosse de la stoa Ouest ! plan et coupe; Éch. 1:20 (Ph. Fraisse).

Pour vérifier une autre hypothèse de $\mathbf{R}$. Vallois ${ }^{\mathbf{b 1}}$, selon laquelle la base rectangulaire située sur l'axe NordSud de la skènè aurait èté celle d'un pilier intérieur faisant partie d'une série de six supports, nous avons implanté une tranchée, large de $1,50 \mathrm{~m}$, de part et d'autre de l'axe Nord-Sud de la skènè (fig. 48). Sur toute sa longueur la tranchée a rencontré, à faible profondeur, la roche granitique. Celle-ci présente une surface plane, qui s'infléchit légèrement ( $d$ 'environ $30 \mathrm{~cm}$ ) dans la partie centrale du bâtiment de scène. La roche n'a conservé aucune trace d'encastrement pour les fondations d'éventuels supports intérieurs, ce qui ne saurait en rien contredire l'hypothèse de $\mathrm{R}$. Vallois car la seule base en place n'est pas encastrée dans le rocher, mais séparée de celui-ci par une mince couche de terre $(5 \mathrm{~cm} \pm)$.

Une seconde tranchèe, large d' $1,50 \mathrm{~m}$, a traversé le bâtiment de scène d'Est en Ouest. Elle aussi a mis au jour le sol granitique à faible profondeur. Dans la stoa Ouest, elle a rencontré, creusée dans la roche, une cavité de forme sommairement rectangulaire $(0,75 \mathrm{~m}$ Nord-Sud $\times 0,95 \mathrm{~m}$ Est-Ouest), à l'angle Nord-Ouest de laquelle aboutit une saignée pentée d'Ouest en Est (fig. 49 et 50). Dans son tiers Ouest, le fond de la cavité est constitué d'un plan situé à $16 \mathrm{~cm}$ en contre-bas de la surface du rocher, qui bute en talus vers l'Est. Les deux tiers restant se trouvent en légère dénivellation par rapport à ce plan, formant une surface dont la profondeur varie entre 19 et $22 \mathrm{~cm}$. Sur l'axe Est-Ouest de ce second niveau se trouve un trou, nettement marqué, de forme approximativement ovale.

En fin de campagne tous les sondages ont été remblayés.

Durant le mois d'aout, deux architectes, B. Mouannes et B. Maurice, ont entrepris, dans le cadre du C.E.A.A., l'étude des accès du thèảtre et des monuments honorifiques installès en bordure du proskènion.

(51) R. VALLoIS, $A H H D, 1$, p. 224-225. 


\title{
4. - Recherches sur le littoral déllen : l'établissement de la "Pointe des pllastres"
}

\author{
par Hervé DuchÊnE
}

\author{
A la mémoire de Nicolas Poffer.
}

Les recherches à la «Pointe des pilastres" se sont poursuivies en 1987. Cette deuxième campagne de fouilles s'est déroulée pendant cinq semaines, du 15 juin au 15 juillet, avec deux ouvriers. Six stagiaires ont en outre participé à ces travaux : Nicholas RauH, enseignant à Trinity College (Université d'Hartford [USA]), Anne Coulie, élève de l'École Normale supérieure, Anne Bénichou et. Dominique GA RCis, étudiants à Montpellier, Nicolas Poffet et Pascal Richoz, de l'Université de Fribourg. Ce dernier a réalisé tous les plans et relevés sur le chantier. Avec son aide, nous avons assuré les prises de vue photographiques.

Les sondages ont été effectués selon un quadrillage défini par rapport aux deux secteurs explorés l'an

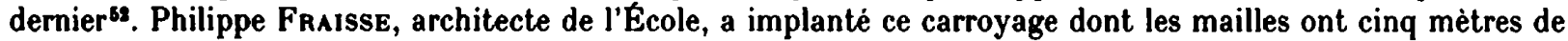
cơté. On a procédé à trois sondages principaux en C5, E5 et D6 (fig. 51). Il convenait cette année de préciser nos hypothèses sur l'occupation de cette région. La campagne précédente avait montré que l'édifice aux pilastres de granit couvrait une superficie beaucoup plus importante qu'on ne le présumait jusqu'à présent. Des réaménagements de la structure initiale étaient nettement apparus. L'ensemble s'inscrivait dans une fourchette chronologique familière à Délos, entre le milieu du ue siècle avant $\mathrm{J}$.-C. et le déclin de l'île, aux environs des guerres de Mithridate.

\section{L'établissement PRIMitif :}

La fouille a confirmé que l'établissement primitif avait été bouleversé par des remaniements récents et multiples. Bien que notre exploration de la première installation ait été limitée, on en a toutefois retrouvé quelques nouveaux vestiges directement fondés sur le substrat naturel. On a pu atteindre, en plusieurs points où la fouille a été complète, soit un niveau de sables d'aspect marin, assez ferme et vierge de toute trace d'habitat antérieur, soit des affleurements de granit servant de fondations et de soutiens aux constructions.

\section{- Sondage en D6.}

Près de la bordure Sud du sondage, on a dégagé un mur de $0,70 \mathrm{~m}$ de large, approximativement orienté Est-Ouest. Par son tracé, il se rattache à la façade de pilastres. Son épaisseur et le bel appareil de blocs de gneiss parsemés de granit témoignent d'une construction solide.

Plus modeste est le mur Nord-Sud situé à la lisière Ouest du même sondage et dont on a suivi le seul parement interne. Les deux murs délimitent unê pièce dont l'extension n'a pu être définie. Elle continue au Nord, hors du périmètre de la fouille; à l'Est, elle a subi de profondes transformations. L'intérieur est très troublé. La couche d'effondrement consiste en gros blocs et tuiles de plusieurs types, analogues à celles rencontrées l'an dernier. Une plaque de tuileaux, posée de chant, appartient à ce remblai, sans que l'on puisse dire qu'elle constituait le sol de cette cellule.

Au sein du mur Est-Ouest, un système d'ouverture fait communiquer cette pièce avec un sol dallé, visiblement extérieur. Il s'agit d'un seuil de gneiss mesurant $1,53 \mathrm{~m}$ de long et $0,65 \mathrm{~m}$ de large. Il porte deux crapaudines de bronze encore en place, la trace d'une feuillure du côté de la pièce et, au milieu, une encoche de verrouillage presque carrée de $8 \mathrm{~cm}$ sur 5 . Le dispositif de clôture est original so. Formé de deux vantaux, il est associé à une fermeture complémentaire en avant, sans doute un demi-battant, dont une cavité rectangulaire de $12 \mathrm{~cm}$ sur 8 à l'angle Ouest serait le témoin. Cet aménagement laisse supposer une fonction utilitaire de l'ensemble. Devant le seuil, plusieurs dalles étaient affaissées; une terre noire mêlée à de la cendre et à des charbons les recouvrait : signes probables d'un incendie. On a démonté deux d'entre elles qui reposaient sur une couche de remblai assez ferme contenant du matériel hellénistique dont deux timbres cnidiens de déchiffrement difficile.

(52) Voir le compte-rendu de la première campagne dans $B C H 111$ (1987), pp. 647-655.

(53) Sur les fermetures déliennes, voir l'essai de Chr. Llinas, dans BCH, Suppl. I (1973), pp. 180-185. Cette réflexion n'envisage que celles des maisons. Nous avons entrepris une ètude des clôtures d'installations marchandes. Les rapprochements les plus instructifs sont à faire avec les magasins de la zone au Sud de l'agora des Compétaliastes. 


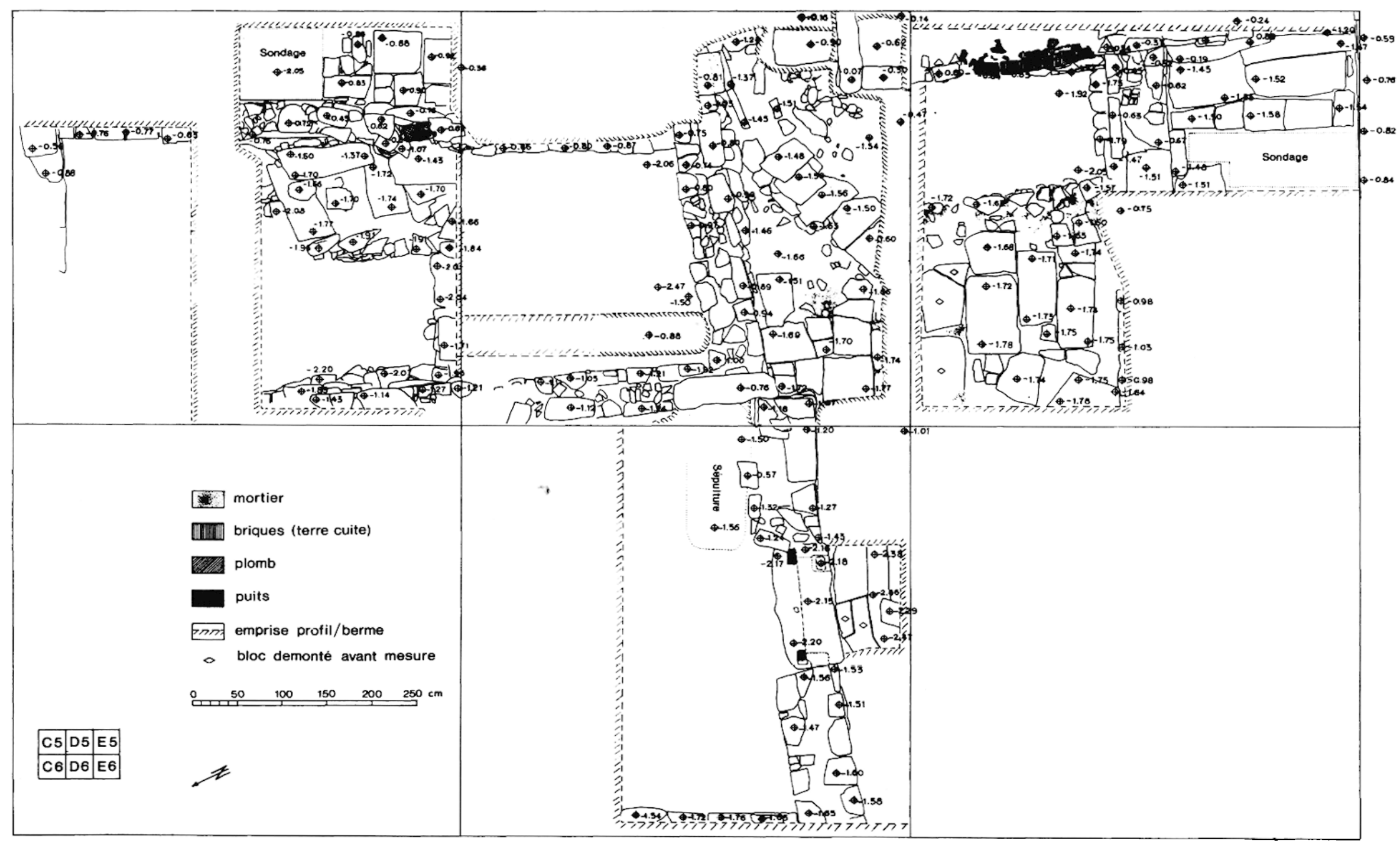

Fig. 51. - Les secteurs fouillés à la Pointe des Pilastres 1986-1987. 
D'un point de vue architectural, la conclusion est malaisée. Le bâtiment a pu s'articuler autour d'une cour à péristyle. Mais il semblerait plutôt s'organiser de façon originale par rapport à une série de couloirs dallés. Perpendiculaires au front de mer, ils ouvriraient sur des pièces disposées latéralement. C'est ce que suggérait déjà $\mathbf{E}$. Ardaillon lors de son relevé de la façade aux piliers de granit ${ }^{\text {th }}$.

\section{- SondAGE EN E5.}

Dans la continuité de celui découvert en 1986 dans le secteur I, mais plus au Sud, dans le sondage en E5, un sol dallé relevant de la première grande phase, était borné à l'Est par un mur plus tard arasé, mais dont on suit encore l'empreinte de la fondation (fig. 52). Au Sud, il est délimité par un mur Est-Ouest de facture soignée, large de $0,70 \mathrm{~m}$. Ce dernier ètait coupé par une ouverture encore inexplorée. Bien qu'approximativement parallèle à celui trouvé en D6, il a un lien direct avec la façade de pilastres et son système de couloirs. Au Sud du sondage, on a d'ailleurs le parement interne d'un nouveau mur parallèle, suivant la même orientation et de construction identique. Il ménage une voie de circulation large de 2,15 $\mathrm{m}$ (fig. 53). Elle s'ouvre au Nord sur l'issue déjà mentionnée et à l'Est, dans l'angle Sud-Est du sondage, sur un passage, large de $0,95 \mathrm{~m}$, en forte dénivellation, qui reste à explorer. Par celui-ci, on communiquait avec un palier supérieur en terrasse dont on a retrouvé la trace en C5.

Toute la zone a fait l'objet d'un drainage soigneux, comme l'atteste l'impressionnante canalisation qui court sous ce couloir dallé (fig. 54). Cet égout est large de $0,70 \mathrm{~m}$ et profond de $0,75 \mathrm{~m}$. Les piles massives qui le constituent sont soigneusement montées, de même que le radier formé de belles plaques jointives. Tout cela traduit une volonté de se protéger de l'humidité et d'éviter les remontées capillaires de l'eau toute proche.

Au vu du matériel exhumé, il semblerait se vérifier que l'ensemble a été aménagé dans le Ire siècle avant notre ère, au moment où Délos devint un port franc. L'activité de ce vaste complexe demeure toutefois encore mal déterminée. Aucun dépôt n'a été conservé en place. Les perturbations causées par les occupations ultérieures empéchent d'assurer, mème si l'agencement général et les dimensions y invitent, qu'il y eut là d'abord une grande aire de stockage.

\section{LES REMANIEMENTS POSTERIEURS.}

La fouille a révélé plusieurs états tardifs. Modifiant la topographie, ils ont cependant repris la disposition de l'édifice étagé en une série de terrasses. Ils ont d'autre part largement utilisé les parties monumentales existantes; le plus souvent, on les a aménagées différemment en les cloisonnant. On n'a pas hésitè toutefois, comme en $\mathrm{C5}$, à reprendre la totalité de l'ouvrage.

Le plan de la pièce dont le dégagement avait commencé en 1986 montre un changement dans l'orientation et une implantation indifférente aux structures les plus anciennes. Leurs sols ont été arrachés et on a fondé directement sur le granit. Un épais remplissage de sable, terre, pierres et gravillons, au matériel très varié, a été jeté pour rattraper les différences de niveau. La pièce grossièrement rectangulaire a sept mètres de long sur trois de large. Une ouverture a été reconnue à l'extrémité Ouest du sondage. Des stucs blancs ou rosés protégeaient les murs dont la construction est hâtive : un empilement de blocs inégaux selon un ordre irrégulier et approximatif.

La vocation marchande est douteuse. Le mobilier recueilli fait penser à une occupation urbaine très fruste. L'aménagement intérieur est énigmatique. Une structure demi-circulaire en appareil règulier provoque un décrochement de près de $50 \mathrm{~cm}$ dans le mur à l'Est du sondage (fig. 55). Une ouverture presque rectangulaire permettait soit le puisage à une réserve, soit le raccordement à un système d'écoulement des eaux venues de la colline. A l'appui de cette suggestion, on notera, le long de ce mur Est, un remblai très massif. Large de 1,50 m, épaisse de $0,75 \mathrm{~m}$, cette banquette inclut dans sa partie haute quelques grosses pierres formant comme un canal. Dans sa partie basse, on trouve une succession assez grossière de lits de pierres. Il fallait se prémunir de l'humidité et consolider la paroi face aux poussées de la terrasse en contre-haut. A l'extérieur de la pièce, la lutte contre le ruissellement est aussi manifeste. On avait repéré l'an dernier dans le secteur I une canalisation aménagée dans le sol dallé de l'établissement primitif ${ }^{\text {ss. }}$. En perturbant encore l'assemblage de dalles et suivant la mème courbe en E5, elle se continue à l'Ouest. Elle passe sous le grand mur Est-Ouest percé à cette occasion et rejoint selon toute vraisemblance le grand émissaire du couloir décrit plus haut. Après un premier examen du matériel, ces modifications se placeraient au rer siècle avant notre ère.

(54) Voir $B C H 20$ (1896), "Rapport sur les fouilles du Port de Délos», pp. $429-445$ et planche I; mais surtout pp. 493-443.

(55) Voir $B C H 111$ (1987), p. 653 et fig. 32 et 39. 


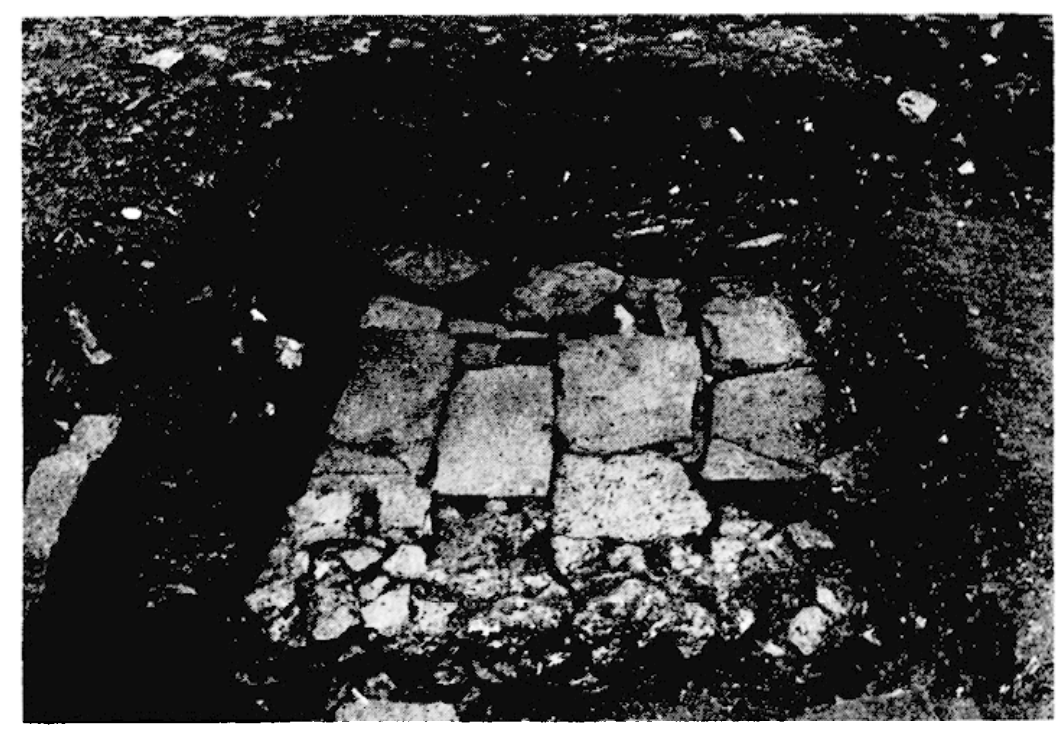

Fig. 52. - Sol dallé et mur arasé en E5.

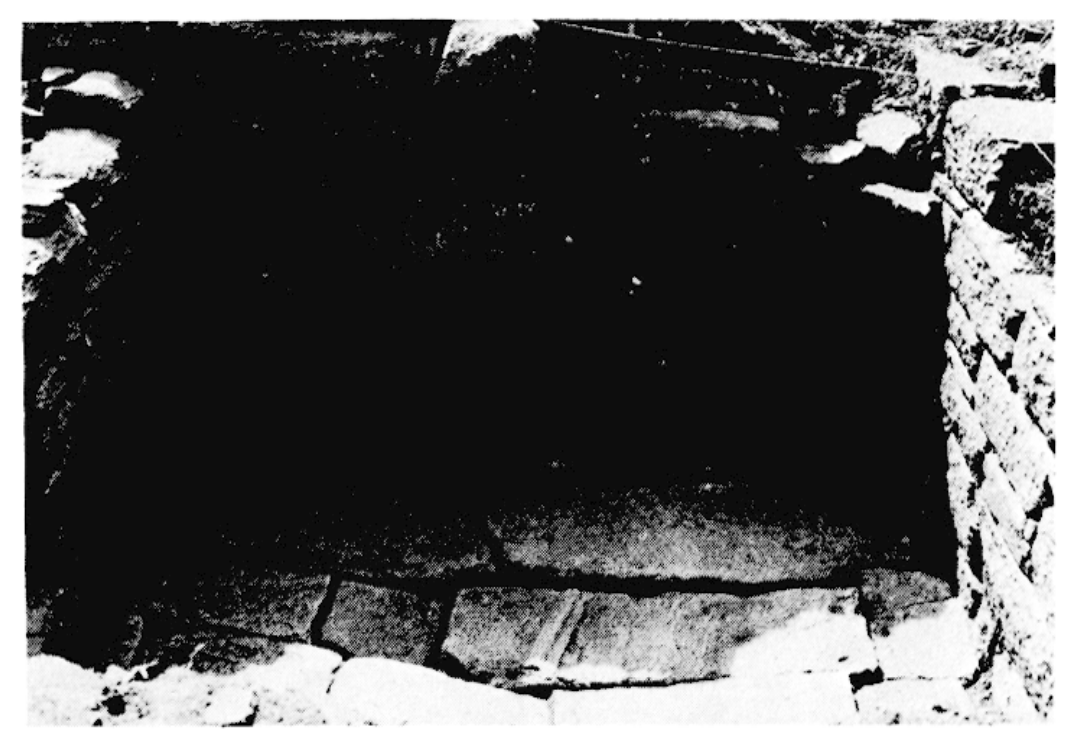

Fig. 53. - Le couloir et les ouvertures à l'Est en E5.

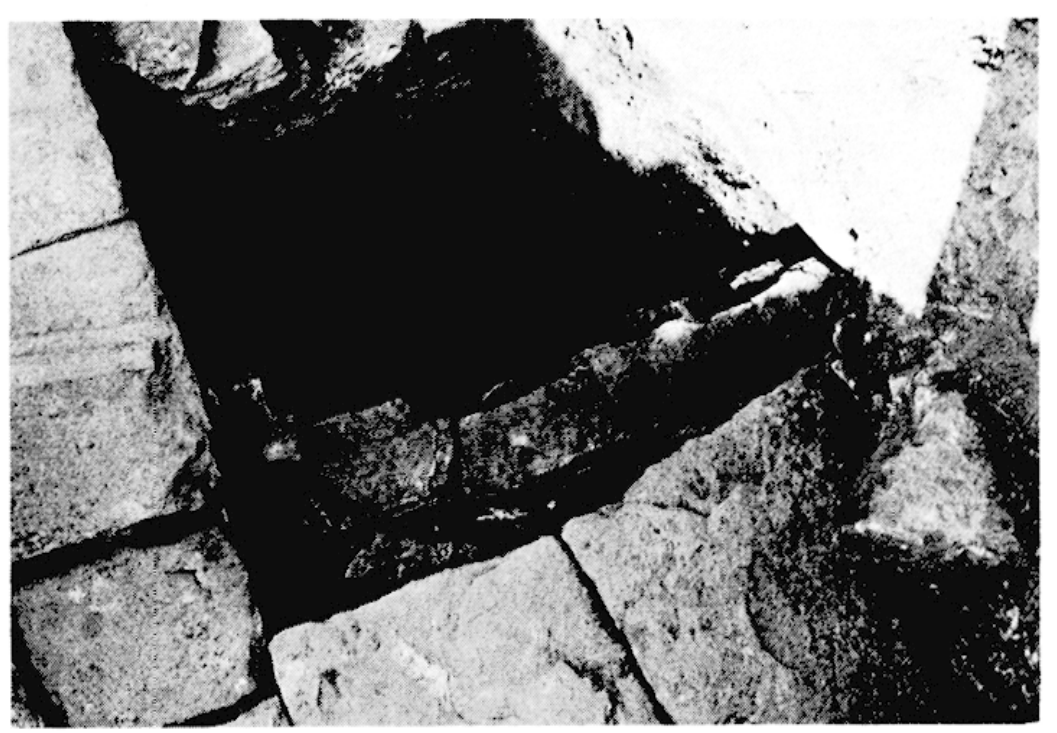

Fig. 54. - La canalisation sous le couloir dallé. 


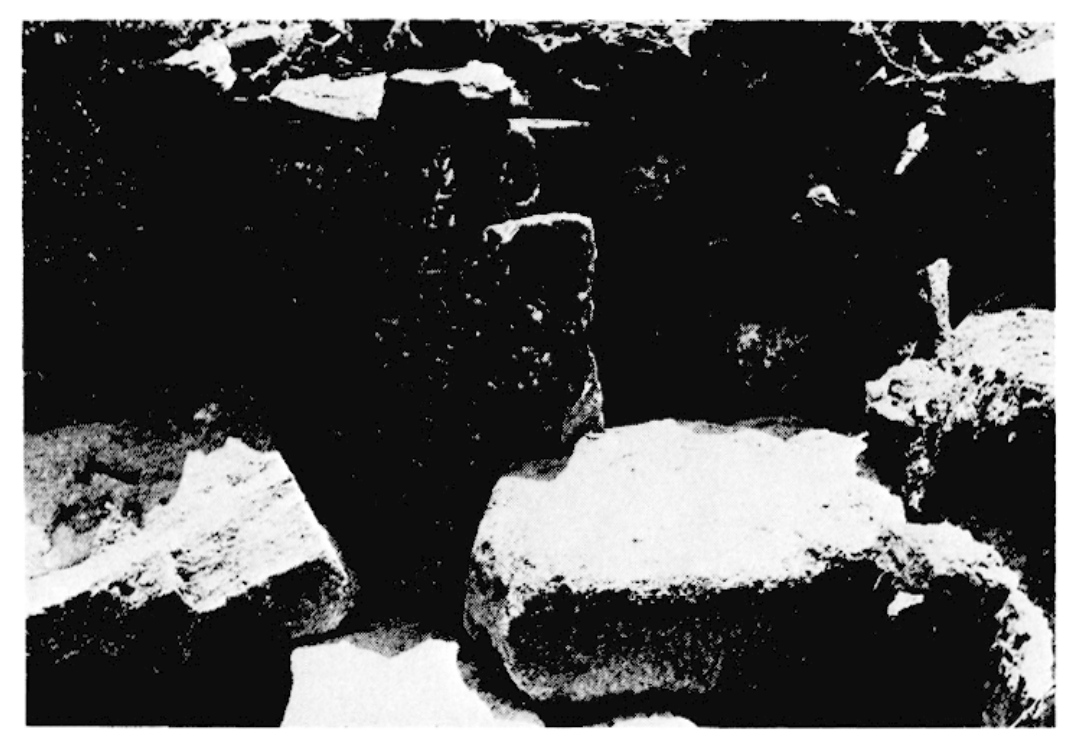

Fig. 55. - La structure demi-circulaire en C5.

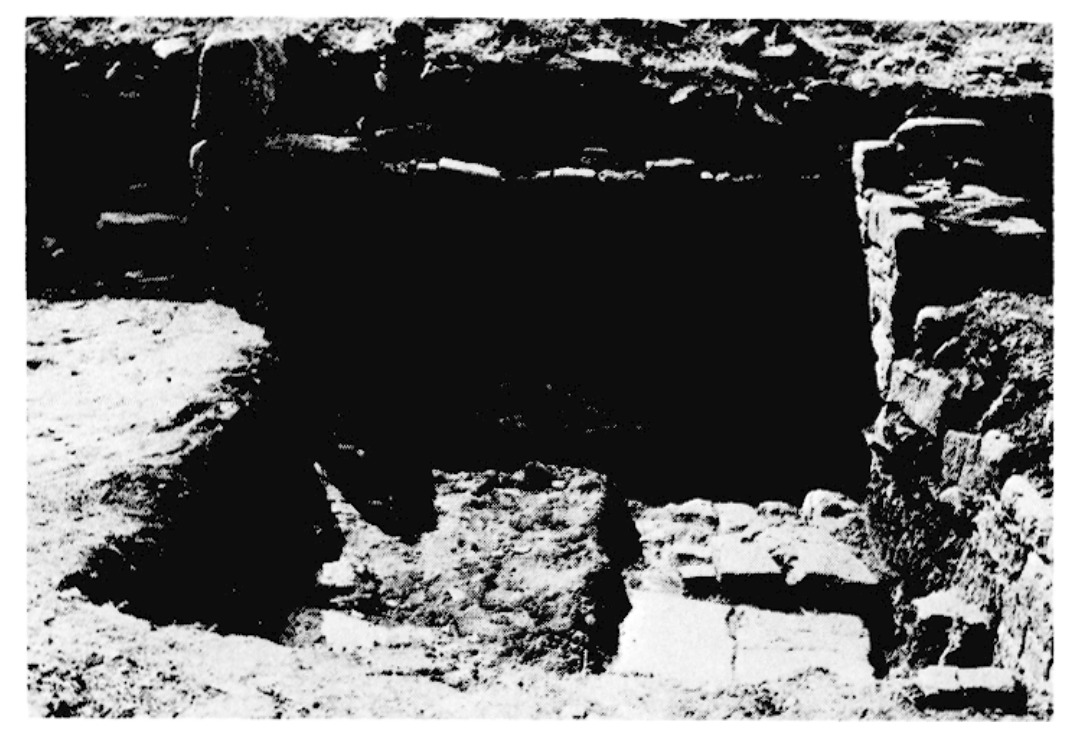

Fig. 56. - Le mur de briques et le réaménagement en $\mathrm{C5}$.

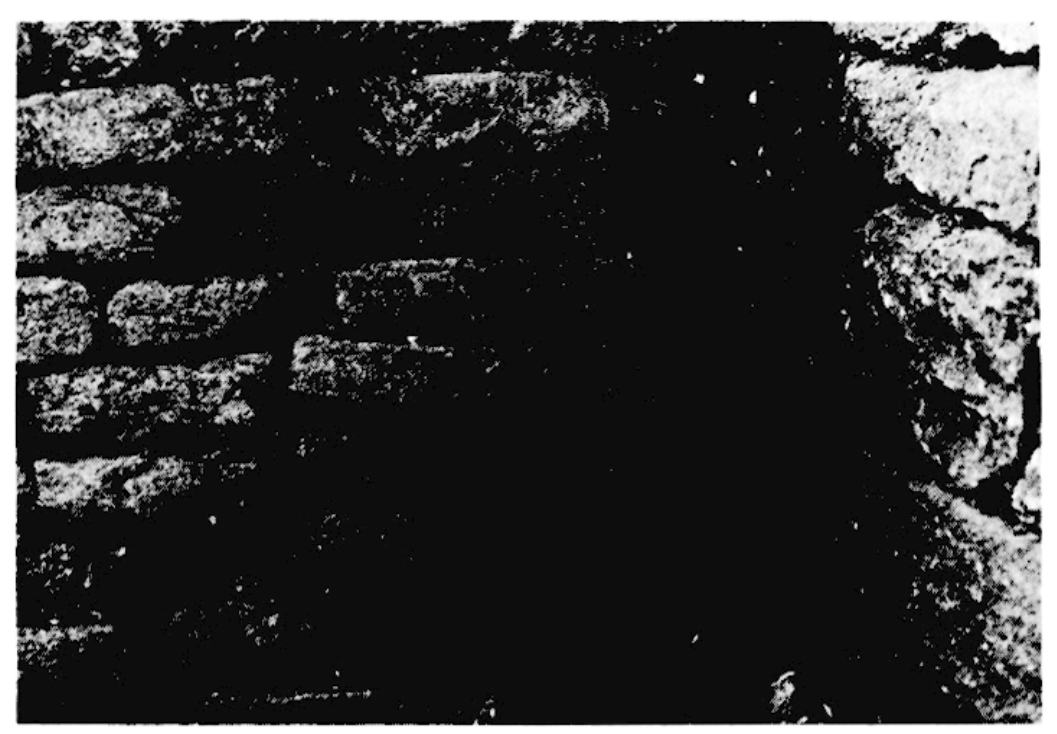

Fig. 57. - Détail du mur de briques avec colombage dans le carré C5. 
Cette réorganisation de la zone paraît relever d'un programme plus vaste. On le croirait après la découverte d'une autre installation réemployant des éléments plus anciens. A l'Est du carré E5, dans le prolongement des deux blocs de granit d'angle repérés en $1986^{56}$, on a mis à jour un mur de briques de terre cuite avec colombage, grossièrement orienté Nord-Sud (fig. 56). Long de deux mètres, c'est le premier aussi bien conservé à Délos. Elévation de briques de terre cuite et piliers verticaux de bois alternent; voilà qui le distingue des murets de terre trouvés ailleurs dans le site dèlien ${ }^{52}$. De texture grossière, ces briques ne portent aucune marque et ne suivent pas un module strict. Larges de $15 \mathrm{~cm}$, elles mesurent en longueur entre 20 et $24 \mathrm{~cm}$ (fig. 57). Elles ont une épaisseur comprise entre 6,8 et $7,2 \mathrm{~cm}$. On peut voir épars à Délos quelques exemplaires de briques strictement comparables aux nótres dans les fouilles Jardé au Nord du "Magasin à la baignoire" et dans le Quartier du théátre. Le mur, fondé sur des blocs de gneiss, prend appui au Nord sur des masses granitiques posées à angle droit, à cheval, et au Sud sur le parement remontant à la première installation. En D6, au bord Sud-Ouest du sondage, on a observé une autre amorce de muret en briques, nouveau cloisonnage du plan originel.

La mise en place de cet appareil de briques et de bois s'est accompagnée d'une destruction des sols et d'un remblayage de toute la zone. Il contenait un matériel abondant, mais fragmenté, de céramique commune hellénistique et d'amphores italiques ou puniques. L'ensemble a un faciès du jer siècle avant notre ère. Au niveau de la fondation, et à la façon d'un dépót, on a recueilli en place deux marmites intactes. L'une, d'argile grisátre, au fond courbe et à la panse globulaire, se caractérise par ses anses : l'une en ruban et l'autre, du côté opposé, parallèle au bord. Ce vase doit être rapproché d'un spécimen absolument identique, lui aussi intact, provenant de la Maison des Tritons dans l'îlot de la Maison des Comédiens ${ }^{58}$. Il se trouvait dans une pièce de service que les fouilleurs ont interprétée comme une cuisine ${ }^{59}$. Un exemplaire similaire, mais de plus petites dimensions, avait été découvert de l'autre côté à l'Est de la pièce $\mathrm{BO}$ de cette même Insula ${ }^{60}$.

Notre trouvaille, mème comparée à celles de l'îlot de la Maison des Comédiens, peut difficilement éclairer la chronologie. Comme le rappelle Ph. Bruneau, les ustensiles de cuisine ont des formes fonctionnelles : elles ont peu varié avec le temps et ne sont d'un point de vue chronologique guère caractéristiques ${ }^{11}$. Ce que l'on sait de I'histoire de l'îlot de la Maison des Comédiens ${ }^{62}$ n'infirme pas en tout cas l'idée que le remaniement à la Pointe des Pilastres a eu lieu dans la première moitié du jer siècle avant notre ère, probablement en son début. Insistons plutỏt sur le fait qu'à ce moment l'occupation à la Pointe des Pilastres se fait sur des bases différentes : la vie marchande a cédé le pas.

En surface, en D6, on a enfin découvert une sépulture. Placée au-dessus des couches d'effondrements antiques, elle est vraisemblablement moderne : ce serait celle d'un noyé inhumé sur place ${ }^{63}$. Le squelette assez gracile, dont il manque le crâne, est allongé sur le dos, bras croisés et orienté Est-Ouest. Cette tombe ne contient aucun matériel.

Fondé sans doute vers 150 , l'établissement de la "Pointe des pilastres" aurait éprouvé des difficultés au début du jer siècle avant notre ère. Il aurait alors perdu sa dimension commerciale pour abriter un foyer urbain médiocre s'éteignant avec la fin de la Délos antique.

(56) Ibidem.

(57) Comme dans le quartier de Skardhana, voir par exemple Ph. Bruneau el alii, EAD 27 (1970), passim.

(58) Voir Ph. Bruneau el alii, EAD 27 (1970), p. 258. Le vase porte le numéro d'inventaire D 212 ; ses dimensions : D. : 22,5; H. : 17.

(59) Idem, p. 98 : "Il faut en tout cas reconnaitre en AI une cuisine*.

(60) Idem, p. 258; numéro d'inventaire : D 213. Un autre modèle (D 214) avec une seule anse en ruban a été trouvé dans le vestibule $A$ de la Maison des Comédiens.

(61) lbidem, p. 255.

(62) D'après Ph. Bruneau, ibidem, pp. 426-427, il fut "abandonné par ses occupants réguliers, vraisemblablement après 88"; l'Insula connut toutefois du cỏté de la rue Ouest "une courte survie en abritant durant quelques années une fabrique de pourpre".

(63) C'était la conclusion de J. Páris qui avait fait des découvertes semblables en 1909, lors de sa prospection du littoral délien. Voir son carnet $D E$ 45, conservé à l'EFA, et sa description d'une sépulture (même orientation et même position) dans la zone juste au Nord de la Pointe des Pilastres. La photothèque de l'EFA conserve deux plaques photographiques, prises vraisemblablement alors et en tout cas dans ce secteur, sous les numéros 2086 et 2087. 


\title{
5. - La rue $\mathbf{V}$ du quartier du Theatre
}

\author{
par Françoise Alabe
}

A la demande de l'Éphorie, et pour préfigurer la desserte par cåble électrique souterrain des maisons de gardiens Sud, il a été procédé à un sondage pour s'assurer de l'existence, de la direction et du point de départ de la rue $\mathrm{V}$ au Sud des parties fouillées. Fr. Alabe et M.-D. Nenna ont réalisé ce sondage avec trois ouvriers, dont deux prètés par l'Éphorie, du 13 au 16 octobre 1987.

L'ensemble de la région est perturbé par des pierriers, des déblais d'anciennes fouilles, de nombreux pans de murs et effondrements : il a donc paru impossible de repérer sur la zone de parcours du cảble ( $350 \mathrm{~m}$ environ) un alignement qui pùt être celui d'une rue dont le point de départ exact n'était pas sûr. En effet, le carrefour des rues $V$ et VI est bouché par un pressoir d'époque impériale, et l'insula non fouillée qui occupe le Sud-Est de ce carrefour présentait deux arrèts de son mur Nord, l'un dans l'axe de la rue V, et l'autre $8 \mathrm{~m}$ plus à l'Est, au droit d'un mur Nord-Sud visible sur une cinquantaine de $m$ dont les 25 derniers sont inclus dans la fortification de Triarius.

Dès lors, il importait de vérifier lequel de ces deux arrêts correspondait à la suite de la rue $V$, et lequel à un démontage postérieur (Triarius ou autre). On a donc implanté une tranchée oblique (larg. $2 \mathrm{~m}$ ) par rapport au réseau apparent, joignant en $11,70 \mathrm{~m}$ le bas du carrefour des rues V-VI au mur récupéré par Triarius. Sitôt apparue dans la partie Ouest de la tranchée une tête de mur alignée sur le retour Ouest et sur les boutiques de la rue V, la partie Est de la tranchée fut laissée de côté, et le carrefour des rues V-VI ainsi localisé dégagé jusqu'au dallage.

\section{Resultats.}

- La rue $\mathrm{V}$ se poursuit vers le Sud dans le mème alignement. Elle est dallée et comporte un égout sur son côté Est.

- Elle est bordée à l'Est d'une rangée de boutiques. Nous avons le seuil (larg. $2,80 \mathrm{~m}$ ) de la première.

- Le mur sur lequel s'appuie la fortification de Triarius est le mur de fond de ces boutiques, situé $8,50 \mathrm{~m}$ en retrait de la rue. Triarius.

- On peut donc postuler que la rue garde ce même alignement jusqu'au retour vers l'Est du mur de

\section{6. - Foullle de la ferme aux Jambages de oranit ot exploration du territolre}

\author{
par Michèle Brunet
}

En mai 1987, une seconde campagne de fouille d'une durée de quatre semaines fut consacrée à la poursuite du dégagement de la ferme aux jambages de granit; y participèrent deux ouvriers et quatre étudiants, A. Franco, J.-Fr. Bichet, O. Borius et D. Renaud.

Plutót que de continuer la fouille selon la méthode des sondages en profondeur adoptée en $1986^{\mathrm{M}}$, il fut décidé de procéder à un décapage du remblai superficiel sur toute la surface de la demeure afin d'en obtenir un premier plan. Quinze sondages furent ainsi successivement ouverts, le dégagement étant systématiquement arrêté au niveau d'apparition des crêtes de murs. On s'est efforcé de maintenir un niveau de dégagement homogène, respectivement dans les parties Nord, centrale et Sud de la ferme, en tenant compte de la déclivité naturelle du terrain et du remblai. On comprendra donc que le plan ainsi obtenu (ig. 58) ne constitue en aucune manière un plan définitif de l'habitation et connaîtra sans nul doute des modifications et des complèments à la lumière des dégagements ultérieurs; il constitue néanmoins un document de travail précieux qui servira de guide pour la poursuite de la fouille.

(64) Voir BCH 111 (1987), Chroniques p. 644-646 pour le compte-rendu de la première campagne. 


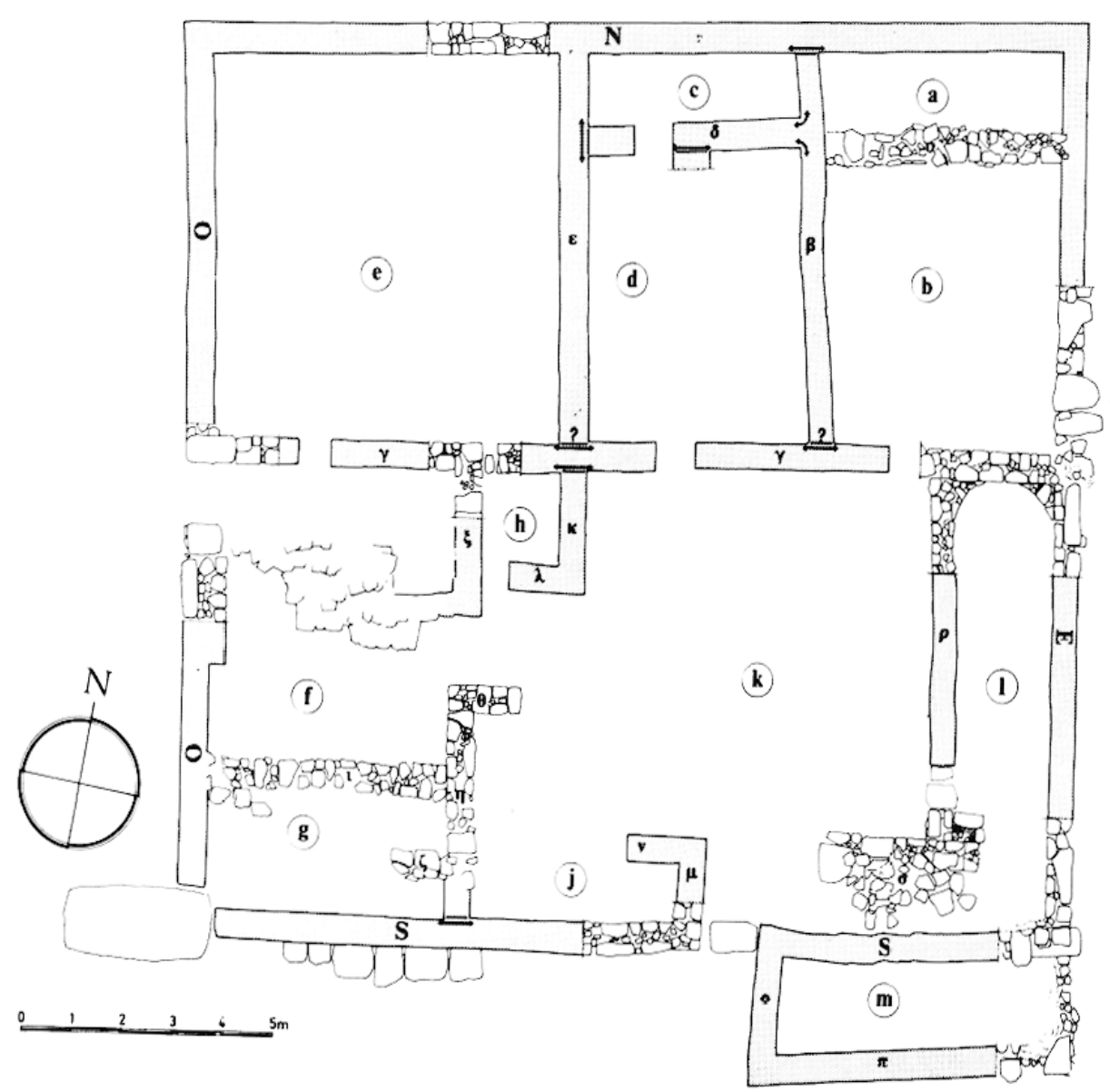

Fig. 58. - Plan de la ferme, $1: 200$ (en l'état du dégagement 1987).

\section{Commentaire du plan.}

La maison disposait de deux entrées larges de $1,10 \mathrm{~m}$, l'une du côté Ouest, l'autre au Sud, entre le retour du mur de façade Sud formant angle avec le mur $\mu^{s 5}$ et le départ du mur o. Elle est flanquée à l'angle Sud-Est par une petite pièce $m$ en saillie de $1,70 \mathrm{~m}$ vers le Sud. Le carré presque régulier dessiné par le bătiment se divise en deux parties nettement distinctes : une partie Nord formée par la juxtaposition de pièces ouvrant toutes vers le Sud et une partie Sud qui semble s'organiser autour d'un espace légèrement décentré interprété comme la cour.

En l'état du dégagement, la moitié Nord semble comporter trois pièces principales, $b$ et $d$ ètant munies d'arrières-pièces $a$ et $c$ très peu profondes, mais il est possible qu'un mur conservé sur une plus faible hauteur et non encore repéré vienne sćparer en deux espaces distincts ce qui apparaît aujourd'hui comme une grande pièce d'angle $e$.

Toute la partie centrale $k$ de la moitié Sud est vide de constructions : il parait raisonnable d'y lire la présence d'une cour. Dans l'angle Sud-Est, une surface de $2,10 \mathrm{~m}$ sur $1,90 \mathrm{~m}$ (massif $\sigma$ ) présente un dallage très irrégulier dont la limite Nord ressemble à un parement de mur. Un dispositif ressemblant à une sortie de canalisation s'observe contre le parement du mur Sud. S'il s'agit bien d'une canalisation traversant ou drainant la cour, elle doit remonter à un état du bătiment antérieur à la construction de la pièce extérieure $m$. Bordant la cour à l'Est, une pièce $l$ longue de $8,30 \mathrm{~m}$ pour une largeur maximale de $2 \mathrm{~m}$ comporte à son extrémité Nord

(65) Les minuscules de l'alphabet grec ont été retenues pour désigner les murs extérieurs de la maison, les minuscules de l'alphabet latin étant réservées aux pièces et les majuscules aux sondages. 


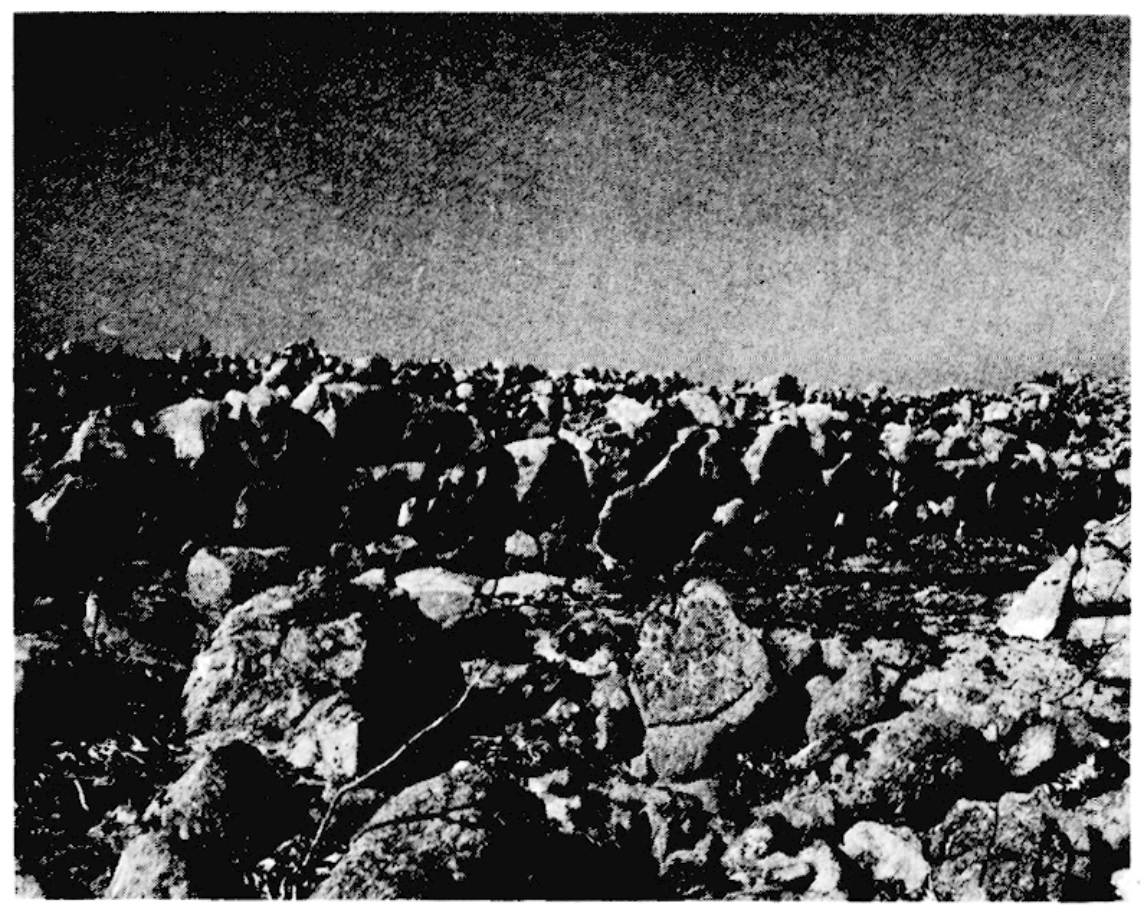

Fig. 59. - Mur de terrasse antique.

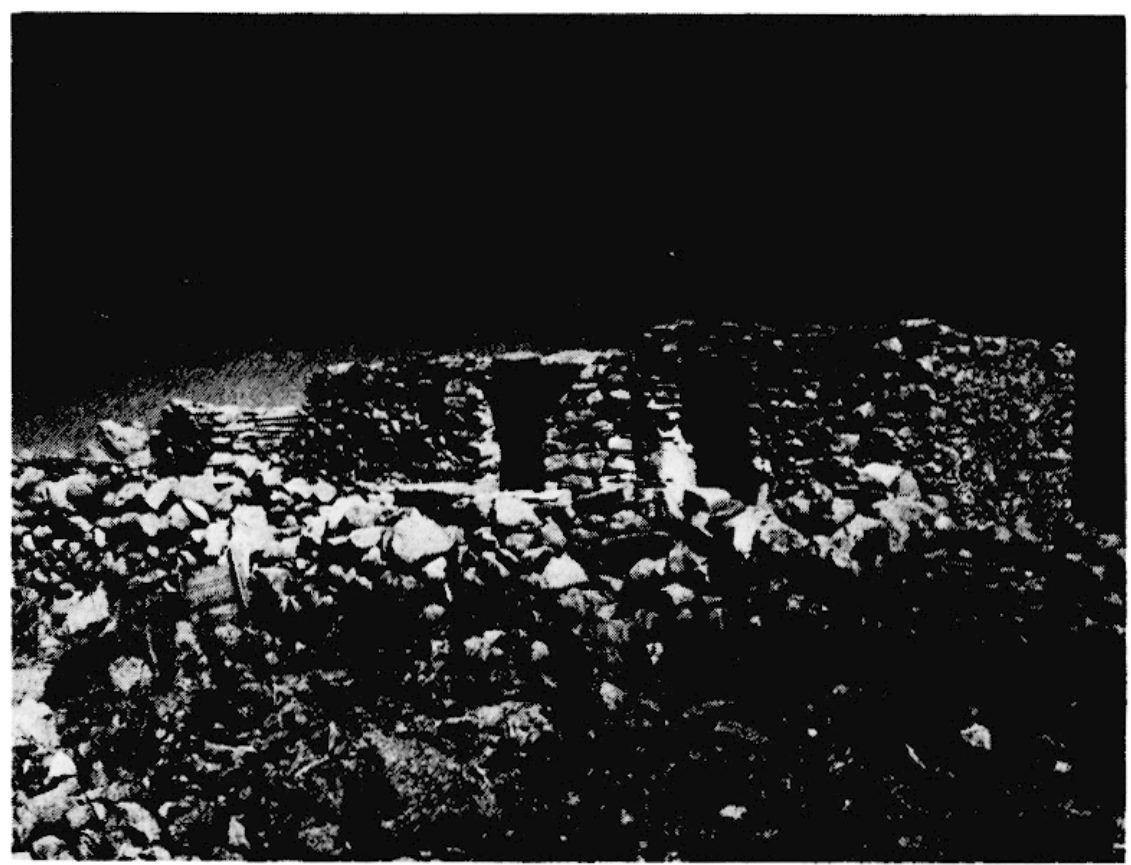

Fig. 60. - Ferme délienne contemporaine. 
une sorte d'kabside inexplicable pour le moment; on accédait à cette pièce par une entrée large de $0,80 \mathrm{~m}$ située entre l'extrémité Sud du mur $\rho$ et le massif $\sigma$. Dans la moitié Ouest de la partie Sud la compréhension du plan est moins directe : l'espace $f$, encombré en son centre par un énorme massif d'effondrement, peut aussi bien constituer une pièce fermée qu'une avant-cour; il est séparé de $g$ par un mur ı dont seul le parement Nord est nettement marqué et dans lequel aucune trace d'ouverture n'est décelable. Du côté Sud, ce mur se fond avec une sorte de dallage très irrégulier reposant sur une couche de terre meuble. A $0,10 \mathrm{~m}$ sous ce dallage, on observe par endroits une mince couche de mortier rosé mêlée à de très nombreux galets marins gris bleuté de taille réduite. Dans l'angle Sud-Ouest, ce ciment repose sur un radier d'éclats de gneiss et de granit : il ne peut s'agir que d'un sol. Enfin, le réduit $h$ mesurant $1,75 \mathrm{~m}$ sur $1,73 \mathrm{~m}$ semble avoir été construit dans une phase ultérieure au plan initial; on y accède par une étroite ouverture de $0,55 \mathrm{~m}$, dans l'axe de ce qui paraît être une porte bouchée dans le mur $\gamma$. La ferme semble donc avoir connu au cours de sa durée d'occupation des modifications de plan avec des ajouts successifs de petites constructions dans le vaste espace vide que devait constituer au départ la cour.

Le matériel présent dans les remblais vient confirmer la chronologie du båtiment avancée l'an passé ; deux dépôts de céramique mêlant toutes les catégories typologiques ont été partiellement dégagés en $j$ et $m$; on y trouve quelques fragments de vaisselle fine attique à vernis noir du ive $\mathbf{s}$. Aucun matériel postérieur au ier $\mathbf{s}$. av. J.-C. n'a èté observé.

Au terme de cette seconde campagne de fouille, la destination respective de chacune des pièces reste énigmatique mais on constate que l'organisation de l'espace intérieur telle qu'elle se présente à ce jour distingue radicalement cette maison des maisons urbaines déliennes; par son plan et ses dimensions, elle se rapproche des maisons d'Olynthe ${ }^{*}$, mais si l'on cherche à la comparer avec les quelques exemplaires de fermes connus dans le monde grec ${ }^{67}$, il appert qu'elle ne se rattache ni au type de ferme de Crimée, ni à celui répertorié en Attique (plans généralement rectangulaires ou oblongs) et absolument pas au type d'habitat fortifié représenté dans les îles.

Le statut de ferme du båtiment ne transparait pour le moment que dans son implantation au milieu de terrasses de culture reconnues pour antiques; aucune des pièces ne semble pouvoir être interprétée comme bergerie ou étable. En corollaire à la fouille, une étude des environs proches de la maison s'imposait donc pour vérifier notre hypothèse d'une répartition des diverses fonctions agricoles entre plusieurs bâtiments et pour mettre en évidence l'organisation des ensembles de terrasses. Deux élèves ingénieurs topographes de l'ENSA IS, 0 . Reis et B. Sastourné ont procédé durant le mois de juillet 87 au levé des terrasses aux abords de la ferme et ont en outre complété le réseau des points d'altitude dans le Sud délien. Le levé topographique a mis en lumière l'existence de trois ensembles de terrasses orientés en harmonie avec le modelé du terrain et de deux chemins sur les lignes d'articulation entre les systèmes; l'un de ces chemins, venant du Sud, conduit à l'entrée Ouest de la ferme (la marche taillèe dans le rocher à l'angle Sud-Ouest montre qu'on avait cherché à faciliter la circulation à cet endroit). La zone immédiatement au Sud du bâtiment semble avoir été aménagée; quelques murs y affleurent en association avec des massifs de granit qui peuvent avoir été utilisés (comme ils le sont encore de nos jours) dans des constructions, formant des fondations ou des pans de murs entiers. En plusieurs endroits au milieu des terrasses, on remarque de tels vestiges, ce qui plaide en faveur d'une dispersion des bătiments agricoles, mais on n'a observé aucun mur de clôture de l'ensemble.

A l'issue de cette deuxième campagne de dégagement, la fouille suggère plus de questions qu'elle n'apporte de réponses aux interrogations suscitées par les inventaires épigraphiques concernant les fermes sacrées : aux problèmes généraux d'exégèse des inventaires viennent se joindre les problèmes particuliers d'interprétation d'un bâtiment ayant manifestement connu plusieurs phases de construction.

\section{Prospection.}

Au cours de l'été, la prospection des secteurs non urbanisés de l'île fut achevée ; au Sud, au-delà d'une zone transitionnelle faisant figure de banlieue où sont juxtaposés vestiges de terrasses de culture et vestiges de vastes demeures de type urbain, sur le pourtour de la baie de Fourni, on aborde proprement la campagne. Les ruines

(66) Cf. D. M. Robinson et J. W. Graham, Excavations at Olynthos VIII, The Hellenic house, 1938.

(67) On se reportera aux planches comparatives illustrant la publication de la ferme de Vari, J. E. JonEs, L. H. Sackett, A. J. Graham, BSA 68 (1973), p. 432; aux fig. 21, 22 et 23 dans J. E. Jones, MIGRA 1 (1975) pour des exemples en Attique et aux plans des bátiments de la presqu'île de Chersonésos dans M. Dufkova et J. Peट̌̇ıKa, Eirene 8 (1970), p. 123-174. 
de dix fermes y ont été repérées, dont trois possédant une aire de battage antique. Les ensembles de terrasses couvrant principalement la façade Sud-Est de l'ile sont incontestablement antiques, comme le prouve le mode de construction des murets (fig. 59); le seul épierrage des surfaces destinées à être cultivées n'a pu suffire à fournir les quantités de matériau nécessaires à cette entreprise gigantesque d'aménagement des pentes et il est plus que probable qu'on eut recours à des blocs extraits de carrière en complément. Au Nord, sur la presqu'île de Patinioti, le substrat de gneiss mis à nu par l'érosion éolienne rend la mise en culture difficile, néanmoins deux grands ensembles de terrasses et d'enclos témoignent d'une occupation antique également dans ce secteur.

La compréhension d'un paysage et de ses phases successives de constitution et d'altération part de l'analyse de ses composantes actuelles confondant en un état unitaire plusieurs états distincts dans le temps (simple transposition à un espace étendu du principe de la stratigraphie); c'est pourquoi il a paru intéressant d'étudier les fermes " modernes " déliennes (ig. 60), afin d'éclairer les permanences dans les choix d'implantation des bătiments, l'utilisation des massifs de granit dans les constructions, la répartition des fonctions agricoles entre plusieurs édifices. A cet effet, Béchara Mouannès, architecte venu à Délos dans le cadre de la formation *Architecture et Archéologie*, a effectué le relevé de cinq de ces fermes durant l'été. 